$\frac{12}{4} \cdot 10.95850$

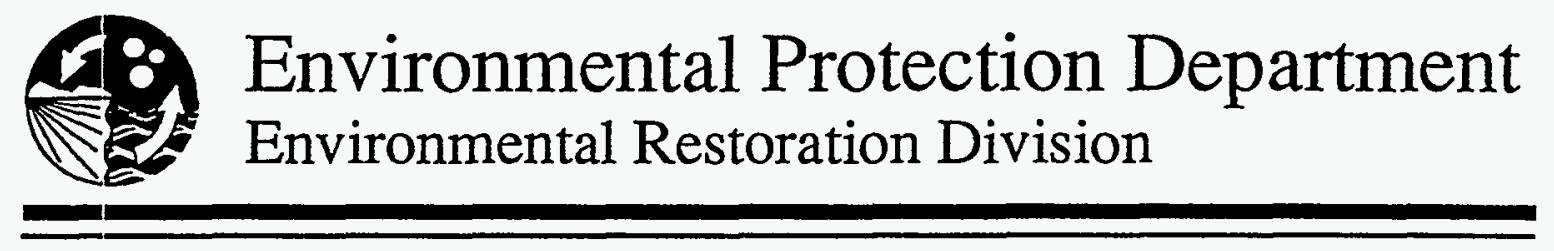

UCRL-ID-116621

\title{
Treatment Facility F: Accelerated Removal and Validation Project
}

\author{
Jerry J. Sweeney \\ Michael H. Buettner \\ Charles R. Carrigan \\ C. Suzanne Chamberlain \\ Alan B. Copeland \\ Maurice A. Hernandez \\ G. Bryant Hudson \\ Marina C. Jovanovich \\ Kevin C. Knauss \\ Ray M. McNairy \\ William H. Siegel \\ Everett A. Sorensen
}

April 1994

Lawrence Livermore National Laboratory

University of California Livermore, California 94551 


\section{DISCLAIMER}

Work performed under the auspices of the U. S. Department of Energy by Lawrence Livermore National Laboratory under contract number W-7405-ENG-48.

This document was prepared as an account of work sponsored by an agency of the United States Government. Neither the United States Government nor the University of California nor any of their employees, makes any warranty, express or implied, or assumes any legal liability or responsibility for the accuracy, completeness, or usefulness of any information, apparatus, product, or process disclosed, or represents that its use would not infiinge privately owned rights. Reference herein to any specific commercial products, process, or service by trade name, trademark, manufacturer, or otherwise, does not necessarily constitute or imply its endorsement, recommendation, or favoring by the United States Government or the University of California. The views and opinions of authors expressed herein do not necessarily state or reflect those of the United States Government or the University of California, and shall not be used for advertising or product endorsement purposes. 


\section{DISCLAIMER}

Portions of this document may be illegible in electronic image products. Images are produced from the best available original document. 


\section{Table of Contents}

Executive Summary (J. J. Sweeney) ................................................................................................... vii

I. Introduction (J.J. Sweeney) .......................................................................................... 1

II. General System Description and Extraction Operations, September 1 to

November 12, 1993 (J. J. Sweeney, E. A. Sorensen, W. H. Siegel) ...........................................6

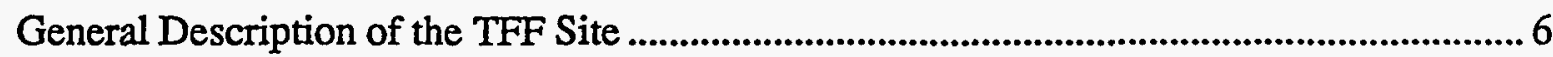

Extraction Operations, September 1 to November 12, 1993 .................................................... 10

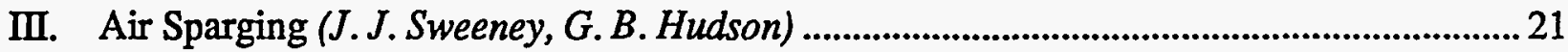

Introduction and General Description ............................................................................................ 21

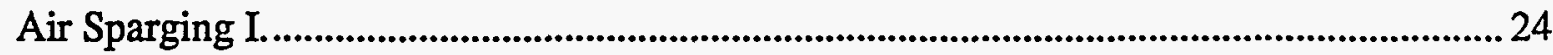

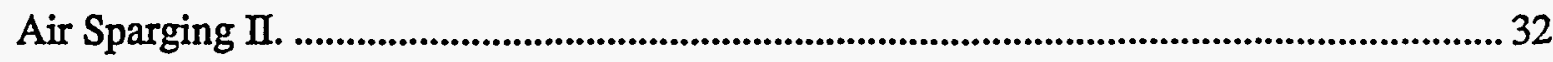

Summary of the Sparging Applications ................................................................................... 40

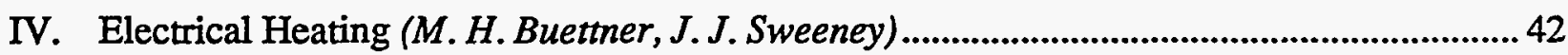

V. Conclusions and Recommendations for Further Work (J. J. Sweeney,

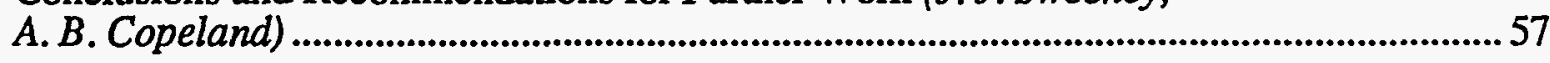

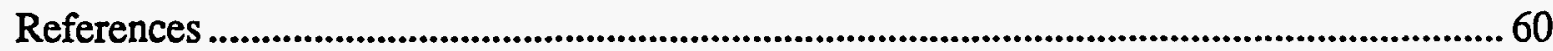

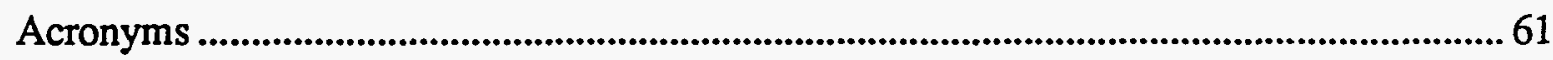

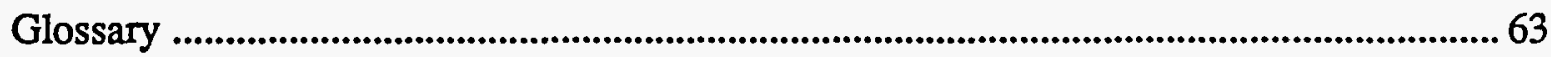

\section{Appendices}

Appendix 1. Accelerated Removal and Validation Project (ARVP) Management (A. B. Copeland)

Appendix 2. System Automation (M.A. Hernandez)

Appendix 3. Electrical Heating Systems (R.M.McNairy, M. H. Buettner)

Appendix 4. Operational Measurements, Sampling, and Chemical Analyses (M. C. Jovanovich, R. E. Martinelli, M.J.Dibley, K.L. Carroll, E. Sorensen)

Appendix 5. Modeling of ARV Sparging and Electrical Heating Using NUFT and an Ohmic Heating Module, and Related Laboratory Measurements (C.R. Carrigan, J.J.Nitao, K. G. Knauss)

Appendix 6. Structure and Flow of Data through the LLNL ERD Environmental Data Management System (C.S. Chamberlain, P.L. Ottesen) 


\section{List of Figures}

Figure 1. Location of wells at the TFF site ...................................................................

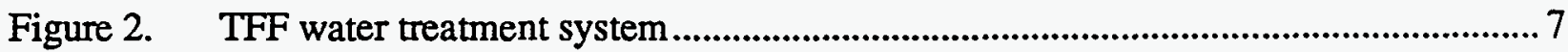

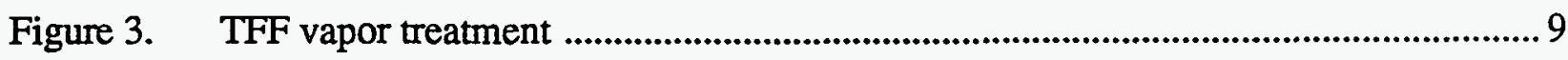

Figure 4. Concentration of total hydrocarbons .......................................................... 11

Figure 5. Plan view of temperature distribution in the upper steam zone .............................. 14

Figure 6. Cross section showing temperature distribution ................................................ 15

Figure 7. Water levels in selected wells versus time during ARV operations ....................... 16

Figure 8. Plan view of the water table at the TFF site as measured on

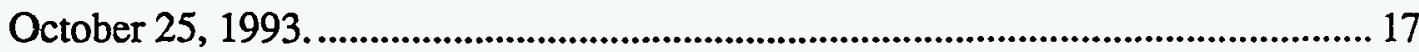

Figure 9. Plan view at the TFF site of the difference between October 4 and October 28, 1993.

Figure 10. View similar to Figure 9, except that the difference in temperatures is between September 15 and October 4, 1993..................................................... 20

Figure 11. Location of wells and ERT profile lines used in the air sparging applications

Figure 12. Calibration curve showing the relation between the air injection rate and the $22 \mathrm{Ne} / 20 \mathrm{Ne}$ ratio. 25

Figure 13. Cross section showing the simplified geology between well GIW820 and GEW808, one of the central extraction wells

Figure 14. Electrical resistance tomography images made from data taken during the first air sparging application

Figure 15. Percent injected air recovered at the upper interval of GIW820 and at the ICE-in versus elapsed time for the second sparging application

Figure 16. Hydrocarbon concentrations in vapor sampled during the second air sparging application 38

Figure 17. Electrical resistance tomography images made from data taken during the second air sparging application.

Figure 18. Plan view of the TFF site showing locations of wells of interest for the electrical heating activities

Figure 19. Same plan view as Figure 18, with the inclusion of path lines showing electrical paths 


\section{List of Figures (cont'd)}

Figure 20. Temperatures at well TEP2 before, during, and after electrical heating phase of ARV.

Figure 21. Temperatures at well TEP7 before, during, and after electrical heating phase of ARV.

Figure 22. Temperatures at well TEP8 before, during, and after electrical heating phase of ARV.

Figure 23. Temperatures at well TEP9 before, during, and after electrical heating phase of ARV...

Figure 24. Temperatures at well TEP10 before, during, and after electrical heating phase of ARV 50

Figure 25. Plot of the rate of condensate recovery in the extracted vapor 52

Figure 26. Cumulative energy deposition into electrodes 53

Figure 27. Simplified diagram of subsurface geology of heater wells. 54

Figure 28. Electrical resistance tomography images made at the end of electrical heating. 


\section{List of Tables}

Table 1. Composition of hydrocarbon vapor samples of injection wells ............................. 12

Table 2. Tracer gas analysis from the first sparging application .................................... 28

Table 3. Hydrocarbon chemistry and cumulative mass removal during first sparging

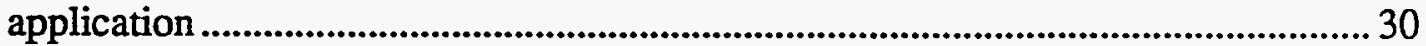

Table 4. Composition of tracer gas during the second air sparging application .................... 34

Table 5. TPH composition from canisters (trace gas samples), second sparging

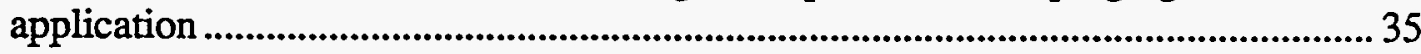

Table 6. Hydrocarbon concentration data during the second sparging application

\section{DISCLAIMER}

This report was prepared as an account of work sponsored by an agency of the United States Government. Neither the United States Government nor any agency thereof, nor any of their employees, makes any warranty, express or implied, or assumes any legal liability or responsibility for the accuracy, completeness, or usefulness of any information, apparatus, product, or process disclosed, or represents that its use would not infringe privately owned rights. Reference herein to any specific commercial product, process, or service by trade name, trademark, manufacturer, or otherwise does not necessarily constitute or imply its endorsement, recommendation, or favoring by the United States Government or any agency thereof. The views and opinions of authors expressed herein do not necessarily state or reflect those of the United States Government or any agency thereof. 


\section{Executive Summary}

The Accelerated Removal and Validation (ARV) phase of remediation at the Treatment Facility F (TFF) site at Lawrence Livermore National Laboratory (LLNL) was designed to accelerate removal of gasoline from the site when compared to normal, single shift, pump-andtreat operations. The intent was to take advantage of the in-place infrastructure plus the increased underground temperatures resulting from the Dynamic Underground Stripping Demonstration Project (DUSDP). Operations continued 24-hours (h) per day between October 4 and December 12, 1993. Three contaminant removal rate enhancement approaches were explored during the period of continuous operation. First, we tried several configurations of the vapor pumping system to maximize the contaminant removal rate. Second, we conducted two brief trials of air injection into the lower steam zone. Results were compared with computer models, and the process was assessed for contaminant removal rate enhancement. Third, we installed equipment to provide additional electrical heating of contaminated low-permeability soil. Four new electrodes were connected into the power system. Diagnostic capabilities at the TFF site were upgraded so that we could safely monitor electrical currents, soil temperatures, and water treatment system processes while approximately $300 \mathrm{~kW}$ of electrical energy was being applied to the subsurface.

The first project activity, commencing on September 1, 1993, was day-shift operation of water and vapor pumping and treating. System automation and upgrades for reliable operations were begun. Training for operators needed for 24 -h operation was conducted. Planning for the electrical heating system was started.

The next activity was institution of $24-\mathrm{h}$ (three shift) operations on October 4, 1993. During this period, water was pumped continuously at a rate of about $50 \mathrm{gal} / \mathrm{min}(72,000 \mathrm{gal} / \mathrm{d})$ simultaneously with vapor extraction at a rate of about 100 standard cubic feet per minute (scfm) (144,000 scfd) from the three central extraction wells at the site. Recovered hydrocarbons (initially about 30 gal of gasoline/d) were destroyed by two internal combustion engines (ICEs), while meeting Bay Area Air Quality Management District (BAAQMD) permitted conditions. Water was treated at the site using an ultraviolet (UV) oxidation system and discharged, after treatment, into the Livermore sewage system. Water was pumped from the lower steam zone (the lower of two zones of high hydraulic conductivity) in order to lower the water table and attempt to drain a confining zone located near the top of the original saturated zone. By draining this confining zone, which was at a temperature of around $90^{\circ} \mathrm{C}$, we hoped to enhance the vaporization of any free-phase, adsorbed, or absorbed hydrocarbons. During the initial 30 days of pumping, the water table was lowered by about $16 \mathrm{ft}$ at the pumping wells.

Between October 4 and November 8, while we were preparing the site for renewed electrical heating, we carried out vapor extraction with two different well configurations. Initially, we used the three central extraction wells for soil venting, switching to four of the steam injection wells (located about $72 \mathrm{ft}$ radially outward from the extraction wells) after 14 days. Vapor flow rates generally were highly dependent upon the well configuration. Each configuration showed a high initial decline in recovery rate that tapered off to a very slow decline. During this period, $830 \mathrm{gal}$ of gasoline was removed as vapor, condensate, and solute. Of this total recovered, about $710 \mathrm{gal}$ was destroyed by the ICEs. 
On November 9, we reverted back to venting from the three central extraction wells to establish a baseline for two periods of air injection (sparging) that were carried out during the weeks of November 15 and 22 . The air injection applications were designed to better understand the processes that occur during air injection both below and above the saturated zone. The measurements addressed four questions: (1) how does injected air behave at the TFF site with various configurations of injection/extraction; (2) can we track injected air with electrical resistance tomography (ERT); (3) how useful are noble gas isotope tracers for monitoring the air injection process; and (4) is air injection a useful means to accelerate recovery of hydrocarbons?

Air was first injected into the lower steam zone (the 112- to 132-ft depth) on November 16 in well GIW820 at a rate of about $45 \mathrm{scfm}$ while venting continued at two of the extraction wells at a rate of about $100 \mathrm{scfm}$. Neon, krypton, and xenon isotope gas tracers were injected into the input air stream. Separate vapor samples were taken at the extraction wells and at an additional nearby monitoring well to analyze for hydrocarbon recovery and for tracer gases. The results generally agreed with modeling that predicted that most of the air would flow radially outward and structurally upgradient from the injection well, with no recovery from the extraction wells. This injection, which lasted $7 \mathrm{~h}$, was terminated once we had confirmed that most of the injected air was not being recovered. Later analysis of ERT images confirmed that the air from the injection well was moving upgradient, away from the extraction wells. The unrecovered injected air was not a major concern because it tends to oxygenate the ground water, thus enhancing microbial activity and hydrocarbon degradation.

The second phase of air sparging took place on November 22 and 23. Air was injected into one steam injection well and extracted from another injection well $65 \mathrm{ft}$ away. In this case, air injection pressures were very close to atmospheric pressure, suggesting that the air was being injected into the vadose zone rather than the saturated zone. This was confirmed after the test, when the water table was found to be at a depth of $119 \mathrm{ft}, 7 \mathrm{ft}$ below the top of the 20 -ft screened interval in the injection well. Trace gas analysis revealed that about $50 \%$ of the injected air was recovered in the extraction well. Analysis of the vapor chemistry was complicated by sampling problems that were identified and corrected during the operation. The concentration of hydrocarbons did not significantly change with time, even though the fraction of injected air in the extract vapor reached as high as $45 \%$. Our interpretation is that the air injection focused the soil venting operation into a zone of higher vapor contaminant concentration near the top of, or within, the low-permeability zone. Both air sparging applications demonstrated the value of modeling and the importance of using tracer gases to better control and enhance the recovery of injected air. During November 8 to 30, an additional estimated 150 gal of gasoline was recovered by the soil venting and water pumping operations at TFF; $75 \%$ of this amount was destroyed by the ICE.

Operations were shut down over the Thanksgiving holiday (November 25 to 28). During this downtime, water levels rose about $6 \mathrm{ft}$ (about 40\%) at the extraction wells. In addition to the activities during October and November, described above, we also completed preparations to resume electrical heating and upgrades to the monitoring system. In order to resume electrical heating, a high-power switching unit was purchased and installed, and power cables were reconnected to heating wells used previously by the DUSDP. New power cables were routed to four new heating wells. These wells were drilled for post-DUSDP characterization analysis and were completed with heating electrodes at depth. 
Operational safety procedures for electrical heating require that any electrically conductive signal wires running between the TFF site and the monitoring facility (which is located outside the personnel exclusion area) be disconnected while electrical heating is in progress. Thus, in prior activities, the water and vapor treatment systems had to be turned off and the well monitoring system disconnected during electrical heating. To overcome this limitation and to enhance the automation of TFF, a fiber-optic (nonconducting) data transmission system was installed so that water treatment operations and well physical parameters (temperature, current) could be continuously monitored while electrical heating was in progress. The entire system was installed and successfully tested by November 24. Electrical heating began on November 30 . The monitoring system performed flawlessly from the very beginning of electrical heating. The ability to continuously monitor the water treatment process system and data from the heating wells and wells located nearby while hundreds of kilowatts of electricity are being applied below the surface represents a significant achievement of the ARV phase of remediation.

Electrical heating started on November 29 and continued through December 13. For safety, electrical heating was carried out only during the swing and overnight shifts, with 24-h operations during the weekend. The effect of the electrical heating on the subsurface was monitored by fixed thermocouples installed in several wells. Signals from the thermocouples and from current sensors at the heater wells were monitored, with the electrical power turned on, by means of the electrically isolated optical fiber monitoring system.

Initially, we could see no effect of the electrical heating on underground temperatures. Analysis of temperature data after completion of the electrical heating showed that some parts of the formation had been heated slightly. These results were very different from those experienced during the electrical heating phase prior to the start of steam injection in the DUSDP. If the ground was not being heated, where was all of the electrical energy going, what was different about this operation? There were several differences. First, we used longer electrodes which extended down into the lower steam zone aquifer, in the new heating wells. Second, we continuously pumped ground water, at a rate of about $50 \mathrm{gal} / \mathrm{min}$, while electrical heating was carried out. Third, we carried out vapor extraction during electrical heating. Focusing on these differences and with the aid of computer modeling, we think we can explain the lack of significant heating of the targeted formation in the ARV electrical heating campaign.

We simulated electrical heating between two electrodes, in geology similar to that at the TFF site, with the three-dimensional NUFT code. The modeling showed a significant amount of heating near heater electrodes. These new, longer electrodes extend into the lower steam zone aquifer where a relatively inexhaustible supply of fresh water is available. High temperatures at the electrode cause the water near it to boil, which produces steam that then migrates upward into the upper steam zone (in the vadose zone) where it comes under the influence of the vacuum from the vapor extraction. Thus, a great deal of energy is lost to latent heat of vaporization near the electrode. This hypothesis is supported by the observation that there was a large increase in the fraction of condensed water recovered in the vapor extraction system, which coincided exactly with electrical heating. In addition to the vaporization near the electrodes, the longer electrode tends to spread the current into several formational units rather than to focus it into the aquitard target zone. This causes a further lowering of heating efficiency. Thus, the longer electrodes installed in the new heater wells provide a combination of effects that results in a much lower heating efficiency. 
On December 9, the water pumps in the extraction wells were shut off and the water table was allowed to rise. During this time, the electrical heating appeared slightly more efficient than when pumps were on. Throughout the electrical heating phase, we observed no increase in the amount of contaminant present either in the pumped water or vapor.

The system was shut down on December 13 and remained idle until mid-January 1994 when single-shift vapor extraction and ground water pumping activities were resumed. With the water again near its unpumped configuration, contaminant levels were much lower in both the vapor and aqueous phases compared to where they were at the start of the ARV operations.

In summary, the main accomplishment of the ARV phase at the TFF site was to remove an additional 1,000 gal or more of gasoline from the subsurface. This is a factor of about two or three higher than what would have been removed under normal operations with a gradually decreasing amount of contaminant present. Vapor concentrations in January 1994 were about 750 parts per million by volume (ppmv) total hydrocarbons (TH) compared to about $6,000 \mathrm{ppmv}$ TH measured in the vapor at the beginning of October 1993. Concentrations of total petroleum hydrocarbons (TPH) in pumped ground water were about $6,000 \mu \mathrm{g} / \mathrm{L}$ in January 1994 compared with about $14,000 \mu \mathrm{g} / \mathrm{L}$ at the beginning of October 1993. Due to the desire to extract maximum contaminant during the course of this project, a definitive experiment correlating water level drawdown with contaminant recovery in the vapor phase was not conducted. This relationship could be useful for future operations and will be determined in the next phase of operation at TFF. Switching vapor extraction to four of the injection wells provided an increase in the contaminant removal rate, demonstrating that air flow patterns in the vadose zone are important for the effectiveness of the remediation. The two air sparging applications demonstrated the utility of noble gas tracer analysis, computer modeling, and ERT imaging for predicting where the injected air will go, and tracking its movement. It was difficult to tell whether the air sparging significantly enhanced contaminant removal rates, but the addition of oxygen into the ground water in the contaminated zone can only help enhance biogenic destruction of gasoline.

The development of the optical fiber monitoring system gave us the ability to safely apply hundreds of kilowatts of power to the subsurface while the TFF site continued to operate under remote control. This is a significant achievement. Even though the electrical heating campaign was unsuccessful in significantly heating the aquitard, we gained insight into some important processes that may be occurring near the heater electrodes. With the aid of computer modeling, coupled with further field trials, we will be able to better understand the near- and far-field processes in electrical heating and how they are influenced by geology and the environment. The predictive capabilities thus acquired will enable us to custom design more efficient systems for electrical thermal remediation. 


\section{Introduction}

The Accelerated Removal and Validation (ARV) phase work described here is the fourth in a series of remediation measures undertaken at Lawrence Livermore National Laboratory (LLNL) Treatment Facility F (TFF), also referred to as the Building 406 Gasoline Spill Area or the Gas Pad. Soil venting, carried out as a pilot study (Cook et al., 1992) between August 1988 and December 1991, removed an estimated 545 gal of BTEX (benzene, toluene, ethyl benzene, and total xylenes), most of which probably came from depths of less than $75 \mathrm{ft}$. The Dynamic Under-ground Steam Demonstration Project (DUSDP) at TFF began operations in December 1992 with electrical heating. The DUSDP Phase I steam injection was carried out between February 3, 1993, and March 14, 1993. In this first phase, gasoline recovery peaked at about 350 gal/d on February 16. An estimated 2,100 gal of gasoline (from vapor, condensate, and ground water) were recovered during this first phase. The third remediation phase at TFF was Phase II of the DUSDP carried out between May 23 and June 30, 1993. An estimated total (vapor + condensate + ground water) of 4,900 gal of gasoline was recovered in this phase. Twenty-four hour extraction continued until July 9,1993 . Intermittent vapor and liquid extraction began on July 29, 1993, continuing until the reinstitution of 24-h/d operation on October 4, 1993. During this period, an estimated additional $1,400 \mathrm{gal}$ of gasoline (recovered total from water, vapor condensate, and vapor) were recovered during single-shift operations.

After the DUSDP and by the beginning of September 1993, the temperatures underground in the steamed areas were still generally above $80^{\circ} \mathrm{C}$, and in many areas above $90^{\circ} \mathrm{C}$. The boiling point of benzene is $80^{\circ} \mathrm{C}$ at one atmosphere (Johnson et al., 1990), so it is probable that any free product remaining in these heated areas will have been substantially stripped of the lighter hydrocarbon fractions. Analysis of soil samples taken from boreholes drilled into the steamed areas in Phases I and II of the DUSDP confirmed that free product had been removed and that residual contaminant levels were very low. However, because of the geometry of the injection well pattern, the relative permeabilities, and the relative rates of steam injection during the DUSDP remediation, an area still remained that was not substantially heated by steam. This "cold spot" occurs within the low hydraulic conductivity zone between the upper and lower steam zones; post-steam injection drilling confirmed that contaminant levels were still high in this zone.

The original volume of the spill at the Building 406 Gasoline Spill Area is unknown. As much as 17,000 gal of leaded gasoline may have leaked from storage tanks at the site between 1952 and 1979 (Devany, 1993). Subsequent weathering and biodegradation has removed an unknown fraction of the gasoline, although we would expect that the lighter fractions would be preferentially removed (Johnson et al., 1990). Devany (1993), using site core sample data and a computer-based volume averaging scheme, estimated 3,890 gal of gasoline in the unsaturated zone and 2,660 gal of gasoline in the saturated zone within the volume of the steam injection ring used by the DUSDP. This estimate did not include free product and is clearly low, since over $7,000 \mathrm{gal}$ of gasoline were recovered during both phases of steam injection, in addition to the estimated 2,200 gal (based on 545 gal of recovered BTEX) of gasoline recovered in the initial soil venting phase and the 1,400 gal recovered during July, August, and September 1993. An additional 1,000 gal of gasoline was recovered during this ARV phase; this sums to a total net 
recovery to date of about 11,600 gal of gasoline. Details of the vapor extraction operations during the period of September 1 through November 11 are given in Section II. Section V addresses the questions: how much gasoline remains to be removed, and where is it? Details of the ARV project planning, scheduling, and cost are given in Appendix 1.

At the beginning of September 1993, the TFF site remained in much the same condition it had been at the completion of steam injection in June, with the exception of the ability to carry out electrical heating and steam injection. The rented boiler was no longer on site, and the LLNL exemption, from the Bay Area Air Quality Management District (BAAQMD), for steam injection had expired. The main power switch for the electrical heating part of the DUSDP was rented for the duration of that part of the project. At the completion of electrical heating, the power cables were cut, and the high power switch unit was returned to the manufacturer. In order to resume electrical heating, the power cables had to be reconnected and the same switch purchased and reinstalled.

One of the aims of this ARV phase of remediation was to enhance recovery of hydrocarbons from the "cold spot" mentioned above. The area had not been heated high enough to completely vaporize all of the free product in the soil, but it had been heated considerably above ambient. Additional thermal remediation of this zone would be most effective and efficient if it were done before the area cooled significantly. The most effective way to enhance recovery in this zone would be to heat the low hydraulic conductivity zone electrically. This contingency had already been planned for because the wells drilled for the post-steam injection evaluation had been completed as heating wells (installation of steel electrodes, thermocouples, and appropriate backfill) that could be used to direct electrical current into the "cold spot." Therefore, one of the initial goals of the ARV phase was to purchase and reinstall the high-power switch, complete power connections to the new heating wells, and reconnect several of the old heating wells so that we could resume electrical heating before the end of December. Details of the installation and design of the electrical heating and monitoring systems are given in Appendix 3. Electrical heating operations and results are discussed in Section III.

We also wanted to continue to pump ground water, carry out vapor extraction and destruction of gasoline vapor, and operate the ultraviolet (UV)/oxidation water treatment system during electrical heating. To do this while applying several hundred kilowatts of electricity to the subsurface, we had to redesign the monitoring sensor systems so that they were electrically isolated from the high-power, 600 -volt heating system. This was accomplished by installing optical fiber links between the monitoring computers, located outside the heater system grid, and the wellheads and the treatment facility where the sensors were located. In addition, the ground water treatment system had to be automated so that system adjustments could be made from outside the site during electrical heating. Details of the system automation are given in Appendix 2; details about the optical system for power isolation are given in Appendix 3.

In addition to the above, it was generally agreed that additional recovery of hydrocarbons remaining in the saturated zone could be enhanced by injecting air, also known as air sparging, into the lower steam zone, below the low hydraulic conductivity aquitard separating it from the upper steam zone (which lies in the vadose zone). Air injected into the saturated zone would presumably move upward to the base of the aquitard and then spread laterally until it eventually found its way, through a fracture or lithologically conductive pathway, into the vadose zone. There, the injected air would eventually be recovered by drawing air from a well or wells 
completed in the vadose zone. As the injected air passes through the ground water, free product or hydrocarbons dissolved in the water will partition into the vapor phase and be carried with the air flow, eventually to be recovered in the extraction well and destroyed. The partitioning into the vapor phase will be enhanced by the high temperatures existing underground; so this process, just like the electrical heating, is best completed before the subsurface cools significantly. The flow of air will depend on factors such as the geometry and lithology of the sediments, relative rates of injection and extraction, and position of the top of the saturated zone. It is very important to know where the air goes and how much is recovered because the air injection could cause contaminants to move away from the recovery area. On the other hand, air injection will result in oxygenation of the pore water. This should enhance microbial activity, leading to the destruction of additional hydrocarbons. For the ARV phase, we designed two simple air injection applications, described in Section III, to better understand the sparging process and its likely ability to enhance recovery of hydrocarbons at the TFF site.

In order to better design the air sparging and electrical heating operations, we carried out some computer simulations with a new code developed at LLNL called NUFT. NUFT is a suite of multiphase, multicomponent models for numerical solution of thermal and isothermal flow and transport in porous media. The code simulates the coupled transport of heat, fluids, and chemical components, including volatile organic compounds (VOCs). The code is a modification of one used to simulate air stripping (sparging) between horizontal wells at the U. S. Department of Energy (DOE) Savannah River Site (Buscheck and Nitao, 1992). Verification and benchmark testing of NUFT have recently been completed (Lee et al., 1993). We used this code to make predictions - which turned out to be very accurate, given the limitations of the model-about what would happen under different situations for the air sparging operations. Under DUSDP, modules were added to the code to enable the simulation of electrical heating. We utilized this added capability to better understand what happened during the electrical heating operations at the TFF site and to help us design follow-up activities. However, in order to do the modeling accurately, we needed to obtain better knowledge of the temperature dependence of the coefficients that describe the solubility and partitioning between the dissolved and vapor phases of the VOCs. For application to this project, we decided that partitioning coefficients for xylene would be the most useful, so a series of laboratory experiments was carried out to obtain the data to define them. Details of the modeling and experiments are given in Appendix 5.

While we planned for the air sparging and prepared to resume electrical heating, we instituted 24-h operations at the site, running the internal combustion engine (ICE) vapor extraction system continuously while continuing to pump and treat ground water around the clock at a rate of about $50 \mathrm{gal} / \mathrm{min}$. At these rates of ground water removal, we were able to lower the top of the saturated zone significantly over a wide area. By draining the rock units, we hoped to increase the volume of sediment accessible to vapor extraction. In addition, with temperatures already at the boiling point of benzene and higher, we could enhance our ability to recover any absorbed, adsorbed, or other free product available. Throughout the ARV phase, ground water was continuously pumped from the central extraction wells (GEW808, GEW816, and GSW16, refer to Fig. 1). We tried two basic configurations of vapor extraction. Initially, we pumped vapor from the central extraction wells, GEW808, GEW816, and GSW16. After about 2 weeks of operation, we switched to vapor extraction from the upper steam zone intervals of the injection 
UCRL-ID-116621

Treatment Facility F:

April 1994

Accelerated Removal and Validation Project

TEP-005

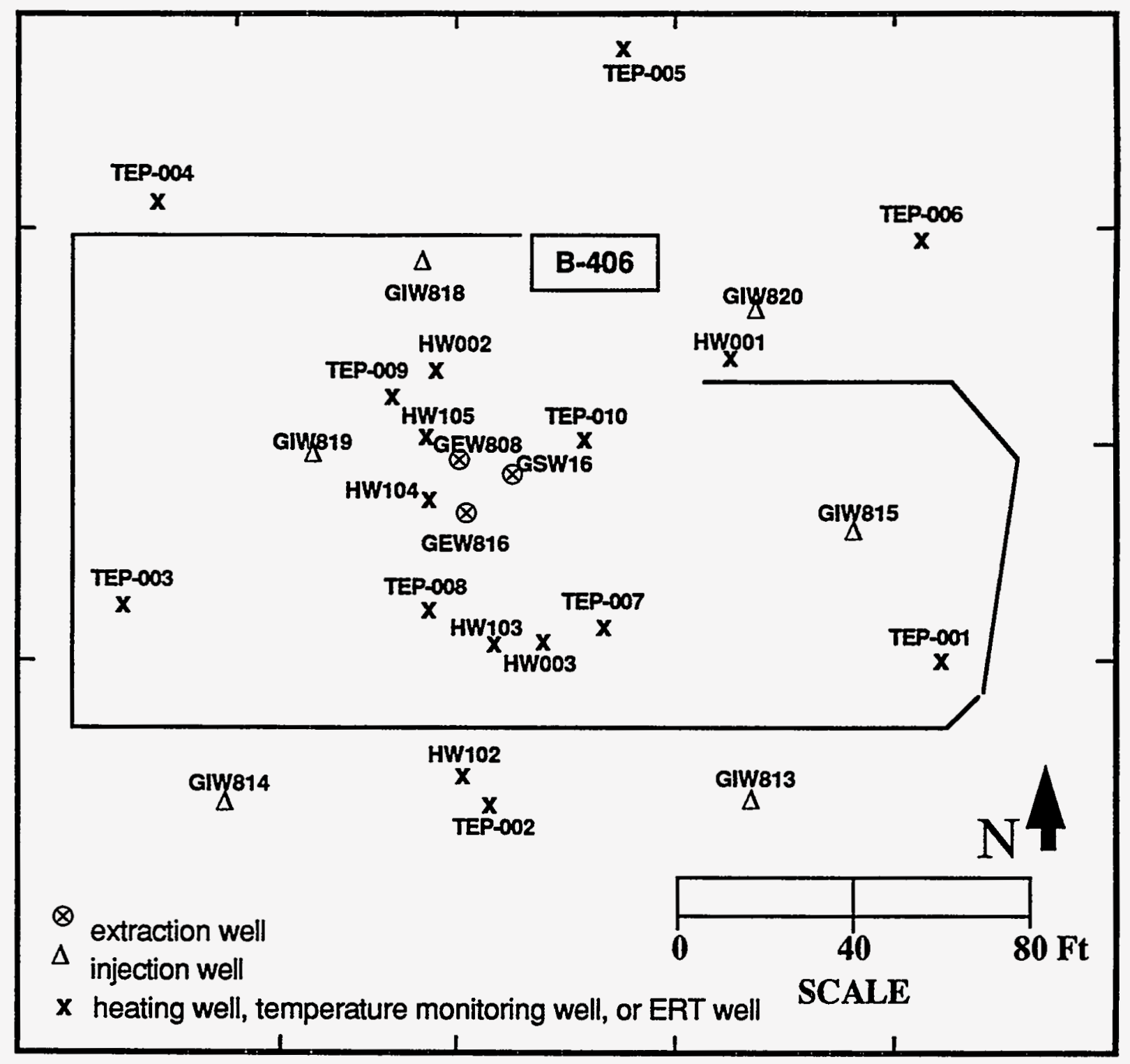

Figure 1. Location of wells at the TFF site.

4 
wells, GIW820, GIW818, GIW815, and GIW813. In both cases, contaminant levels rapidly dropped off from initial highs with the removal rates decreasing with time. Details of these operations are given in Section II.

We end this introduction with a note of discussion (or caveat) concerning sampling of hydrocarbons and estimates of gasoline recovered. During the ARV phase, $75 \%$ or more of the hydrocarbons recovered were in the vapor phase. Condensate and hydrocarbons dissolved in water made up the rest. Therefore, estimates of recovery of vapor phase product drive the estimate for total product recovered. Sampling of vapor and testing for hydrocarbon concentrations is time-consuming and, hence, expensive. For these reasons, sampling for hydrocarbon concentrations was generally done only once a day. This single sample was then used, along with the average daily volume of vapor extracted, to estimate the total daily vapor recovery. Subdaily fluctuations in the contaminant level of the extracted vapor will not be detected this way, and we depend on our past experience during the DUSDP to support the assumption that the recovered sample is representative of the daily average. Potentially, recovery rates can also be monitored by tracking the amount of natural gas burned by the ICE (natural gas consumption increases as the vapor concentration decreases), but this correlation is not very sensitive to concentration variation at the concentrations seen during this phase and was not useful here.

The fact that we are forced to obtain vapor samples from a system under vacuum introduces additional difficulties, as we observed during the second air sparging operation (discussed in Section III). When sampling from a system under vacuum, there is always the possibility that air other than from the vapor stream can leak into the sampling system and dilute the sample. If this happens, the measured contaminant level will always be less than the true level and the corresponding estimates of recovery will be low. We found that improvements in our sampling technique and system were needed and these were made. Because of this possibility, the levels of product recovery reported herein should be considered as minimum values because any errors in sampling will tend to be on the low side. The issues of sampling, sample analysis, and data management are discussed in detail in Appendices 4 and 6. 


\section{General System Description and Extraction Operations, September 1 to November 12, 1993}

\section{General Description of the TFF Site}

TFF was originally designed to remediate soil and ground water by a combination of soil venting and conventional pump-and-treat technology. Some components were added or modified to accommodate the DUSDP, but the facility remains, basically, a combination of a ground water treatment system and a vapor removal and treatment system. These two systems are briefly described below.

The ground water treatment system (Fig. 2) consists of five components: (1) extraction wells and pneumatic pumps, (2) an ambient-air heat exchanger, (3) oil/water separators (OWS), (4) a UV/oxidation system, and (5) air stripping tanks with granulated activated charcoal (GAC) treatment. Each of these systems are briefly described below.

Ground water is pumped at about 190 liters per minute (L/min, $50 \mathrm{gpm})$ from three centrally located extraction wells (GEW808, GEW816, and GSW16) with three air lift pumps. These pumps were chosen because of potentially explosive downhole conditions and to minimize emulsification of contaminant in ground water. The combined maximum yield from the pumps, located $42 \mathrm{~m}(138 \mathrm{ft})$ below ground surface, is about $230 \mathrm{~L} / \mathrm{min}(60 \mathrm{gpm})$. Extracted ground water is cooled, from a range of $90^{\circ}$ to $93^{\circ} \mathrm{C}\left(195^{\circ}\right.$ to $\left.200^{\circ} \mathrm{F}\right)$ to about $38^{\circ}$ to $40^{\circ} \mathrm{C}\left(100^{\circ}\right.$ to $110^{\circ} \mathrm{F}$ ), with an ambient-air heat exchanger to prevent thermal degradation of the treatment facility's equipment. Water is then routed to an OWS to remove free-phase gasoline. Even though free product was observed in monitoring wells historically, only about $700 \mathrm{~L}$ (180 gals) of free product out of a total recovered gasoline volume of about $26,000 \mathrm{~L}$ (7,000 gals) were skimmed from ground water early in the DUSDP. No free product has been recovered directly from extracted ground water since March 1993.

The TFF water treatment system includes a Peroxidation Systems, Inc., model LBV-60, $60 \mathrm{~kW}$ UV light/hydrogen peroxide $\left.\mathrm{H}_{2} \mathrm{O}_{2}\right)$ oxidation system. Although UV/oxidation can be cost-effective, we experienced reduced hydrocarbon destruction efficiency with the system during and immediately after the DUSDP steam injection. We hypothesize that UV light absorption by aqueous inorganic compounds may have reduced available UV light. In order to deal with some of the single-bonded carbon VOCs, such as chloroform, carbon tetrachloride, and ethylene dibromide, which are poorly degraded in the UV/oxidation process, and to achieve regulatory discharge standards in treated water, a final air stripping process is utilized. After this use, the gas used in the air stripping process is routed through GAC. This treated air then meets BAAQMD requirements of a maximum 10 parts per million by volume (ppmv) total hydrocarbon concentration.

Although TFF was designed to meet National Pollutant Discharge Elimination System (NPDES) requirements, trace levels of some VOCs and some inorganic compounds in the treated 


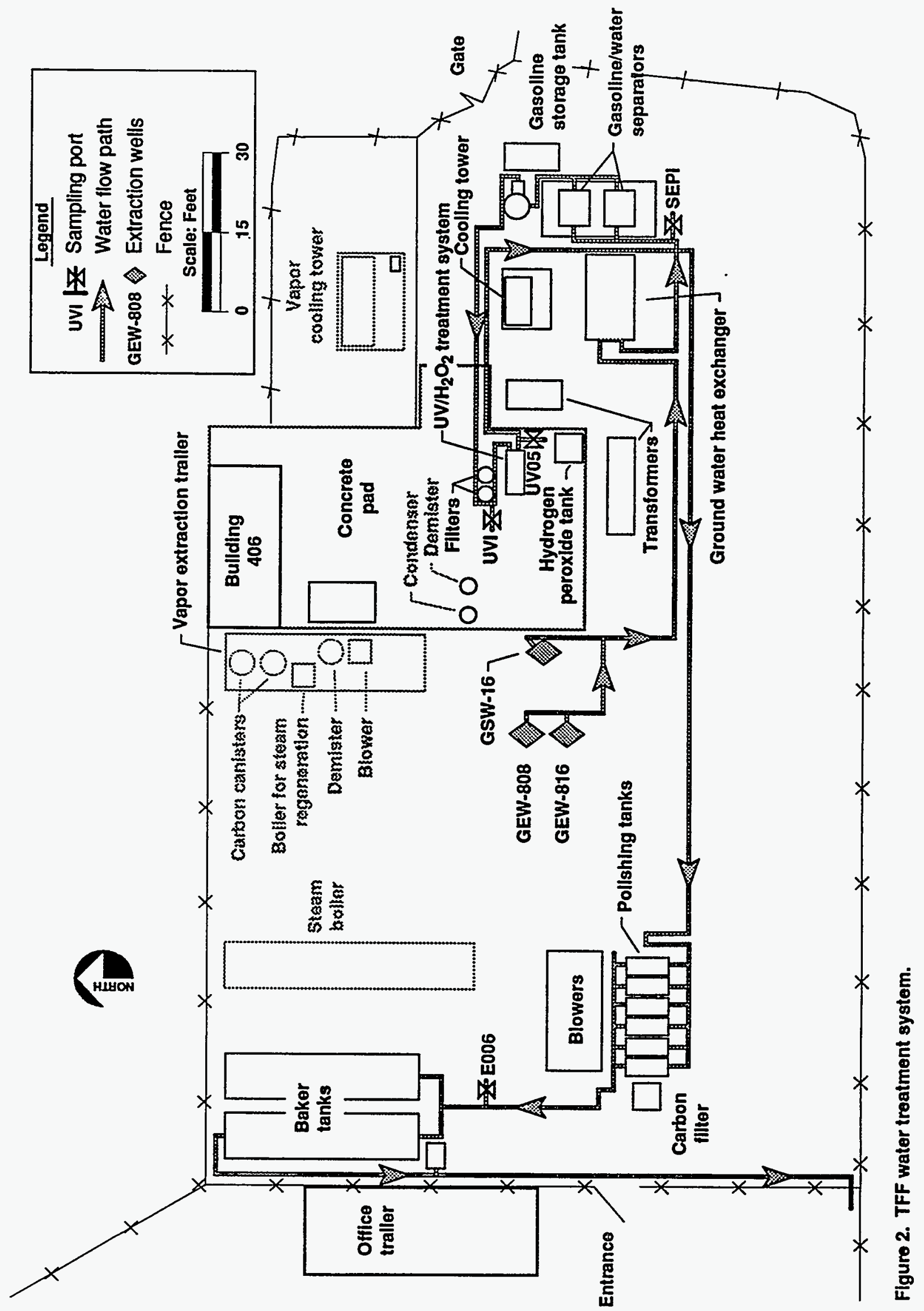


effluent were detected at slightly elevated concentrations during the DUSDP. In order to proceed with the DUSDP, permits were obtained to discharge treated water to the sanitary sewer. Because these permits were still in effect during the ARV project, we continued to exercise this option.

The vapor treatment system at TFF (Fig. 3) consists of (1) extraction wells, (2) a flat plate heat exchanger, (3) a condensate treatment system, (4) an internal combustion engine system for hydrocarbon destruction, and (5) an activated charcoal adsorption system. Each of these system components is briefly described below.

The vapor treatment system extracts vapor primarily from the same three centrally located extraction wells as the ground water treatment system. These wells are screened in the vadose zone, beginning about $20 \mathrm{ft}$ below the ground surface. Extraction vacuum is provided by the VR Systems ICE, described below. In addition, we also used the DUSDP steam injection wells, which surround the central extraction wells in a six-spot pattern (the GIW wells of Fig. 1), as supplemental vapor extraction points during the course of the ARV phase of remediation.

During and after the DUSDP, extracted vapor temperatures rose as high as $90^{\circ} \mathrm{C}\left(195^{\circ} \mathrm{F}\right)$. In order to safely handle this vapor, it is first cooled in a flat-plate heat exchanger. Steam (which comprised up to $90 \%$ of the extracted vapor during DUSDP) and gasoline are condensed into liquid in the heat exchanger. Thus, two process streams leave the heat exchanger; cooled vapor and condensed fluid. Condensed water and gasoline are pumped to a 20-gpm Megator OWS. Gasoline is skimmed and the remaining water is pumped into the ground water treatment system just upstream of the UV/oxidation system. The condensed fluid is free of particles and microbes, both of which can foul conventionally operated OWSs. The maximum gasoline skimming rate from this unit was about $90 \mathrm{~L}$ ( 24 gals) of gasoline per day during the DUSDP second "steam pass" in June and July 1993.

For the direct treatment of vapor, Continental Recovery Systems (CRS) of Natick, Massachusetts, designed and built a self-regenerating GAC adsorption system for LLNL. One of two $340-\mathrm{kg}$ (750 lb) GAC vessels is used to adsorb extracted vapor while the other vessel, which will have been saturated by prior use, is desorbed using the unit's $100 \mathrm{~kW}(400,000 \mathrm{BTU} / \mathrm{h})$ steam generator. Regenerated vapor, consisting almost entirely of steam and gasoline vapor, is condensed in a small flat-plate heat exchanger and separated in an OWS within the unit. This system can collect about $100 \mathrm{~L}$ ( 30 gals) of gasoline per day.

Hydrocarbon concentrations in the extracted vapor increased from around 2,500 ppmv total hydrocarbons (TH), measured in early February 1993, to as high as 100,000 ppmv TH in April 1993, after the DUSDP first steam injection phase. To increase the gasoline removal capacity above the CRS trailer's 100-L (30 gal)/d limit, we installed a VR Systems ICE capable of extracting and burning as much as $600 \mathrm{~L}$ (150 gals) of gasoline per day. The stand-alone unit consists of two 7.5-L (460 in. ${ }^{3}$ ) displacement Ford industrial engines controlled by a microprocessor. The unit automatically blends dilution air into extracted vapor to maintain excess oxygen in the exhaust's catalytic converters. 


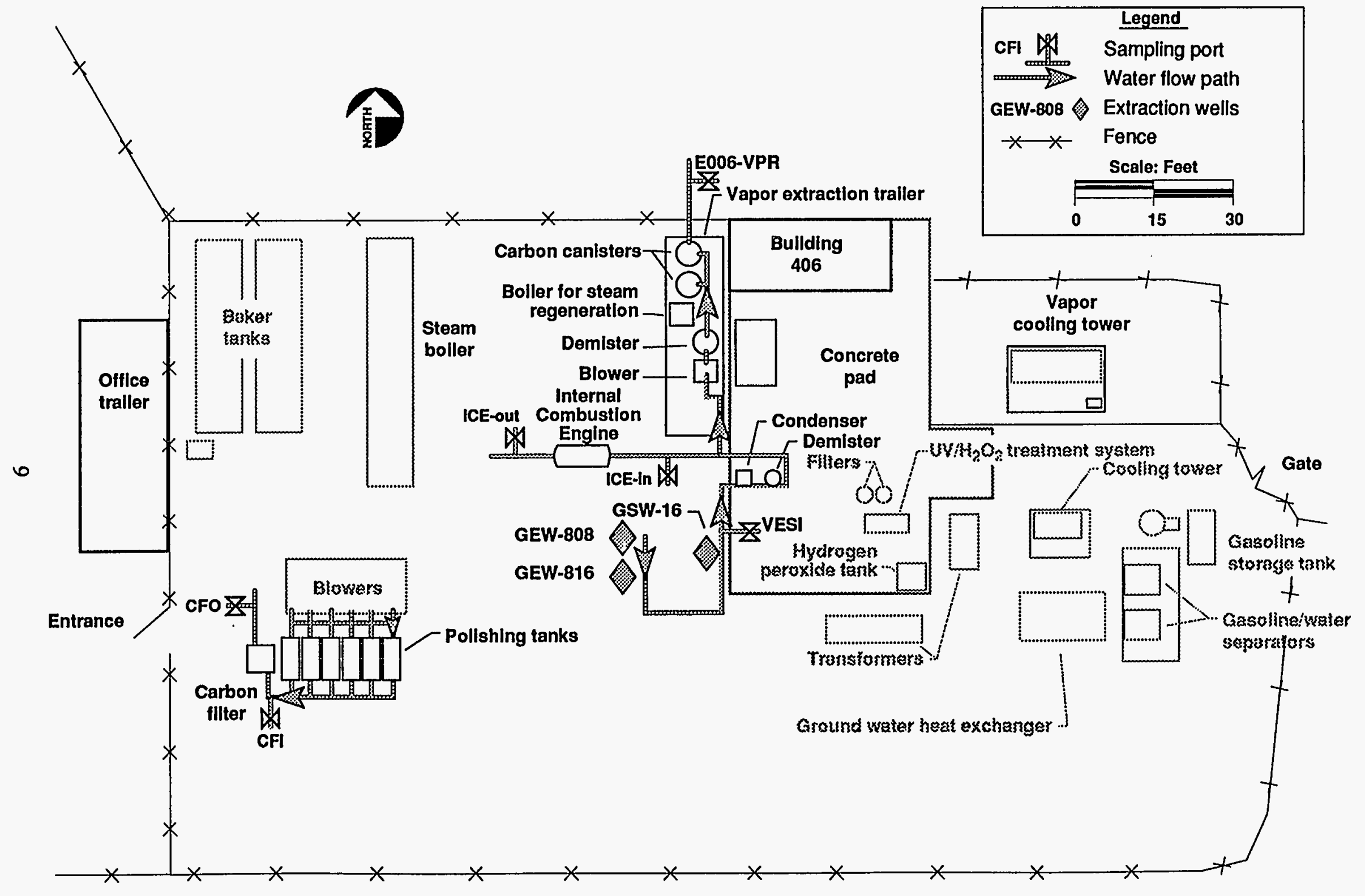

Flgure 3. TFF vapor treatment. 
Both the vapor and water treatment systems at TFF are operated and controlled by an IBMtype 486-microprocessor-based computer. Although the facility typically operates near steadystate, many operational parameters are monitored by the computer. If any of the parameters fall out of the planned normal range, the computer is capable of shutting down the entire system and alerting an operator. The controlling computer also allows automatic data-logging of process parameters.

\section{Extraction Operations, September 1 to November 12, 1993}

During September 1993, vapor and water extraction and treatment were carried out on a oneshift-per-day basis for 12 days, with a total operating time of $78 \mathrm{~h}$. Daily hours of operation ranged from 5 to 8 and averaged 6.5. Approximately 174,000 gal of water and $840,000 \mathrm{ft}^{3}$ of vapor were removed and treated at the TFF site. This corresponds to removal rates of $34 \mathrm{gal} / \mathrm{min}$ and $165 \mathrm{scfm}$, respectively. Initial concentration of THs, as measured at the input to the ICE where the gasoline vapors are destroyed, was $21 \mathrm{mg} / \mathrm{L}(6,000 \mathrm{ppmv}$ at STP) on September 1 . The last ICE measurement for the month, taken on September 22 , was $17 \mathrm{mg} / \mathrm{L}(4,800 \mathrm{ppmv})$. Concentrations of THs in the water averaged about $18,000 \mathrm{ppb}$. A total of $26 \mathrm{gal}$ of gasoline was recovered from the pumped water, 19 gal as condensate, and 167 gal were destroyed or recovered from the vapor extraction for a total removal of $212 \mathrm{gal}$.

Three-shift, 24-h, 7-days-per-week operation of the water pumping and treatment system and the ICE vapor extraction/destruction system commenced on October 4, 1993 (Julian day 277). Water pumping throughout the entire ARV phase came from the three central extraction wells, GEW808, GEW816, and GSW16 (Fig. 1). Initially, vapor extraction was carried out from these same wells from screened intervals covering a large portion of the vadose zone between $20 \mathrm{ft}$ and the top of the water level in the well, which ranged from 102 to $122 \mathrm{ft}$ in depth. Average extraction rates were about $100 \mathrm{scfm}$ for vapor and about $50 \mathrm{gal} / \mathrm{min}$ for water. As seen in Figure 4, initial concentrations of THs, sampled at the input to the ICE, were 16 to $19 \mathrm{mg} / \mathrm{L}$ $(4,500$ to $5,400 \mathrm{ppmv})$. These levels decreased rapidly to about 7 to $9 \mathrm{mg} / \mathrm{L}(2,000$ to 2,500 ppmv) during the next 2 weeks of operation.

The initial hydrocarbon concentration of $18 \mathrm{mg} / \mathrm{L}(5,200 \mathrm{ppmv})$, at $100 \mathrm{scfm}$ extraction rate, means that about $26 \mathrm{gal} / \mathrm{d}$ were being destroyed. As the concentration dropped to half of this level, $9 \mathrm{mg} / \mathrm{L}(2,600 \mathrm{ppmv})$, we were recovering about $13 \mathrm{gal} / \mathrm{d}$. At these relatively low rates, we were not using the ICE very efficiently as a recovery/destruction system. In addition, because of the wide depth range of screened intervals in the extraction wells, we knew we were not focusing the flow of vapor into the most highly contaminated parts of the vadose zone. The injection wells have screened intervals confined to the upper steam zone of the vadose zone. We, therefore, decided to try to increase the recovery rates by shifting the vapor extraction to several of the injection wells (GIW813, GIW815, GIW818, and GIW820) shown in Figure 1, which had good vapor flow characteristics.

Initially, GEW808 and GEW816 were taken out of the vacuum stream and GSW16 was left in when the injection wells were connected to the ICE on October 18 (day 291). Vapor in the injection wells was extracted from a 20 -ft screened interval in the upper steam zone aquifer, located in the vadose zone at depths of 67 to $105 \mathrm{ft}$ (different for each well). As seen in Figure 4, initial concentrations for the composite of all these wells, as measured at the input to the ICE, 
Кер/Іе6 'рәлошәл ОН

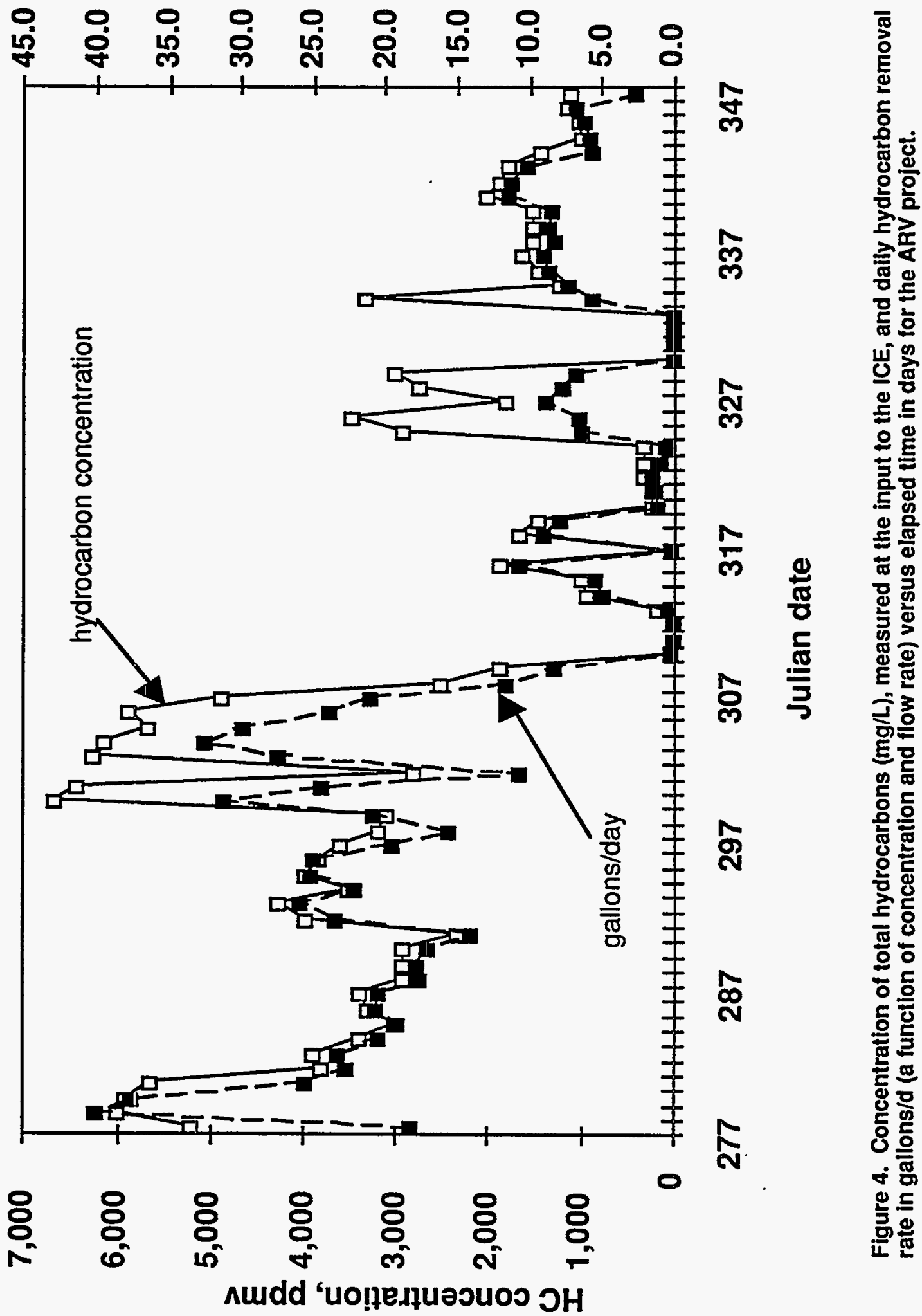


went up to 10 or $11 \mathrm{mg} / \mathrm{L}$ (about $4,000 \mathrm{ppmv}$ ). There was a considerable dilution effect from the lower concentrations in GSW16; this was revealed when we took GSW16 off-line on October 26 (day 299), and concentrations at the ICE input (Fig. 4) jumped back up to $18 \mathrm{mg} / \mathrm{L}(6,600 \mathrm{ppmv})$ when we were only extracting from the four injection wells. Coincident with the jump in concentration was a drop in the rate of vapor flow by about one-third from the four injection wells (about 120,000 scf/d) compared to the previous case when GSW16 was included (about $180,000 \mathrm{scf} / \mathrm{d})$. Apparently, about one-third of the flow in the vapor system had been coming from GSW16.

Contaminant levels in each of the four injection wells were measured on October 21 (day 294) and again (after GSW16 was isolated) on October 28. Results are shown in Table 1. A flow test completed on October 28 (day 301) on each of the four injection wells revealed that about $50 \%$ of the flow was coming from GIW815, with the rest equally divided (at 16 to $17 \%$ each) between the other three wells. Partitioning the concentrations for October 28 according to the flow distribution gives a total concentration in the flow of $6,360 \mathrm{ppmv}$. This number agrees well with average concentrations of the composite flow measured at the input to the ICE for that general time period.

Vapor extraction continued from the injection wells until November 5 (day 309) when the entire TFF system was shut down in preparation for a weekend power outage. As seen in Figure 4 , concentration levels declined gradually between October 18 and November 5 . In preparation for the air sparging operations (described in Section III), on November 8 (day 312) we returned to extraction from the three central extraction wells after the power outage. We expected that hydrocarbon concentrations at the input to the ICE upon resumption of extraction from these wells would be comparable to or higher than the values measured on October 17, prior to switching to extraction from the injection wells. Instead, concentration levels (Fig. 4) were much lower, in the range of $1,000 \mathrm{ppmv}$ or less instead of the expected 3,000 to 4,000 ppmv. It is likely that these values were anomalously low because of sampling problems (Section III and Appendix 4). This phase of operations ended on November 16 (day 320) with the beginning of air sparging operations, described in the next section. In addition to changes in hydrocarbon concentrations, operations from October 4 to November 11 affected the level of the water table and subsurface temperatures under TFF; these changes are described below.

Table 1. Composition of hydrocarbon vapor samples of injection wells.

\begin{tabular}{|c|c|c|c|c|}
\hline \multirow[b]{3}{*}{ Well ID } & \multicolumn{4}{|c|}{ Total petroleum hydrocarbon content in vapor } \\
\hline & \multicolumn{2}{|c|}{ October 21, 1993} & \multicolumn{2}{|c|}{ October 28, 1993} \\
\hline & $\begin{array}{c}\text { TPH } \\
\text { (mg/L air) }\end{array}$ & $\begin{array}{c}\text { TPH } \\
\text { (ppmv) }\end{array}$ & $\begin{array}{c}\text { TPH } \\
\text { (mg/L air) }\end{array}$ & $\begin{array}{c}\text { TPH } \\
\text { (ppmv) }\end{array}$ \\
\hline GIW-813 & 44.0 & 12,580 & 49.6 & 13,840 \\
\hline GIW-815 & 13.0 & 3,670 & 15.3 & 4,270 \\
\hline GIW-818 & 7.7 & 2,190 & 18.8 & 5,250 \\
\hline GIW-820 & 32.0 & 9,010 & 22.0 & 6,130 \\
\hline
\end{tabular}


Temperatures were measured throughout the TFF site at different depths from a network of thermocouples installed for the DUSDP. These thermocouples were continuously monitored by the data acquisition system throughout the ARV phase. From these data, we estimated the subsurface thermal conditions at the site, and their changes over time. In addition, continuous measurements of water level were made in the extraction wells, where the pumps were operated continuously, and daily water level measurements were made in seven surrounding wells. From these data, we more precisely monitored changes in the water table (or hydraulic head) that resulted from pumping at rates that averaged $50 \mathrm{gal} / \mathrm{min}(72,000 \mathrm{gal} / \mathrm{d})$.

Figure 5 shows in plan view the temperature distribution on October 5 at the depth of the upper steam zone (80-ft or $24.4-\mathrm{m}$ depth). This is the area most strongly affected by steam injection during DUSDP activities in March through July. Monitoring well TEP010, which is at a temperature of $96.5^{\circ} \mathrm{C}\left(206^{\circ} \mathrm{F}\right)$ at the $24.4-\mathrm{m}$ depth, is located close to the extraction wells and in the center of the steam injection ring used in the DUSDP project. The temperature distribution was controlled by the steam injection process and lithology. Permeable zones through which most of the steam passed were heated to near boiling temperatures, while less permeable zones, mainly above and below the level shown in Figure 5, remained cool. This can be better seen in the cross section in Figure 6, which shows that the highest temperatures were confined to levels between $70 \mathrm{ft}(21.3 \mathrm{~m})$ and $120 \mathrm{ft}(36.6 \mathrm{~m})$. This encompasses the upper and lower steam zones. Note that by October, 3 months after the end of steam injection, underground temperatures were still high and the clay aquitard layer, which is at a depth of about 100 to $115 \mathrm{ft}$ (30.5 to $35 \mathrm{~m})$, had been heated by conduction to temperatures similar to those in the upper and lower steam zones. Aside from changes associated with ground water flow, the temperatures shown in Figures 6 and 7 decreased by only by $1^{\circ}$ to $2^{\circ} \mathrm{C}$ during the entire ARV phase.

Water levels measured in the five wells at the TFF site during the ARV phase are shown in Figure 7 . The trends are complicated by various system configuration changes that were made throughout the course of the project, but the decline curve due to pumping during the first 2 weeks, while vacuum was simultaneously applied to the wells where water pumping was occurring, is readily seen. As expected, water levels dropped by 2 to $3 \mathrm{ft}$ when vacuum was shut off in the extraction wells (around Julian day 291) because of the increased air pressure in the wells. Figure 8 shows the configuration of the water table (since the water is being pumped from the lower steam zone aquifer, it more accurately represents a surface of constant head in the lower steam zone) under the TFF site after 21 days of continuous pumping (on October 25). The original water table was at the 102- to 104-ft (31.1- to 31.7-m) depth; in Figure 8, the water table is at depths greater than $112 \mathrm{ft}(34.1 \mathrm{~m})$ over a large area. Note also the cone of depression around the extraction wells. This cone is not symmetric; it is flattened on the western side probably due to permeability variations. More closely spaced water level contours occur in areas of lower permeability; this suggests that most of the flow in the lower aquifer is from the southeast. Note that this flow direction also corresponds to the axis of elongation of the $95^{\circ} \mathrm{C}$ contour in Figure 5; this is because the temperatures are controlled by preferential paths for steam, which follow the direction of maximum permeability. The real significance of Figure 8 is that it shows that we were successful in drawing the water table to depths within or below the depth of the clay aquitard, which was the target zone for enhanced contaminant recovery. 


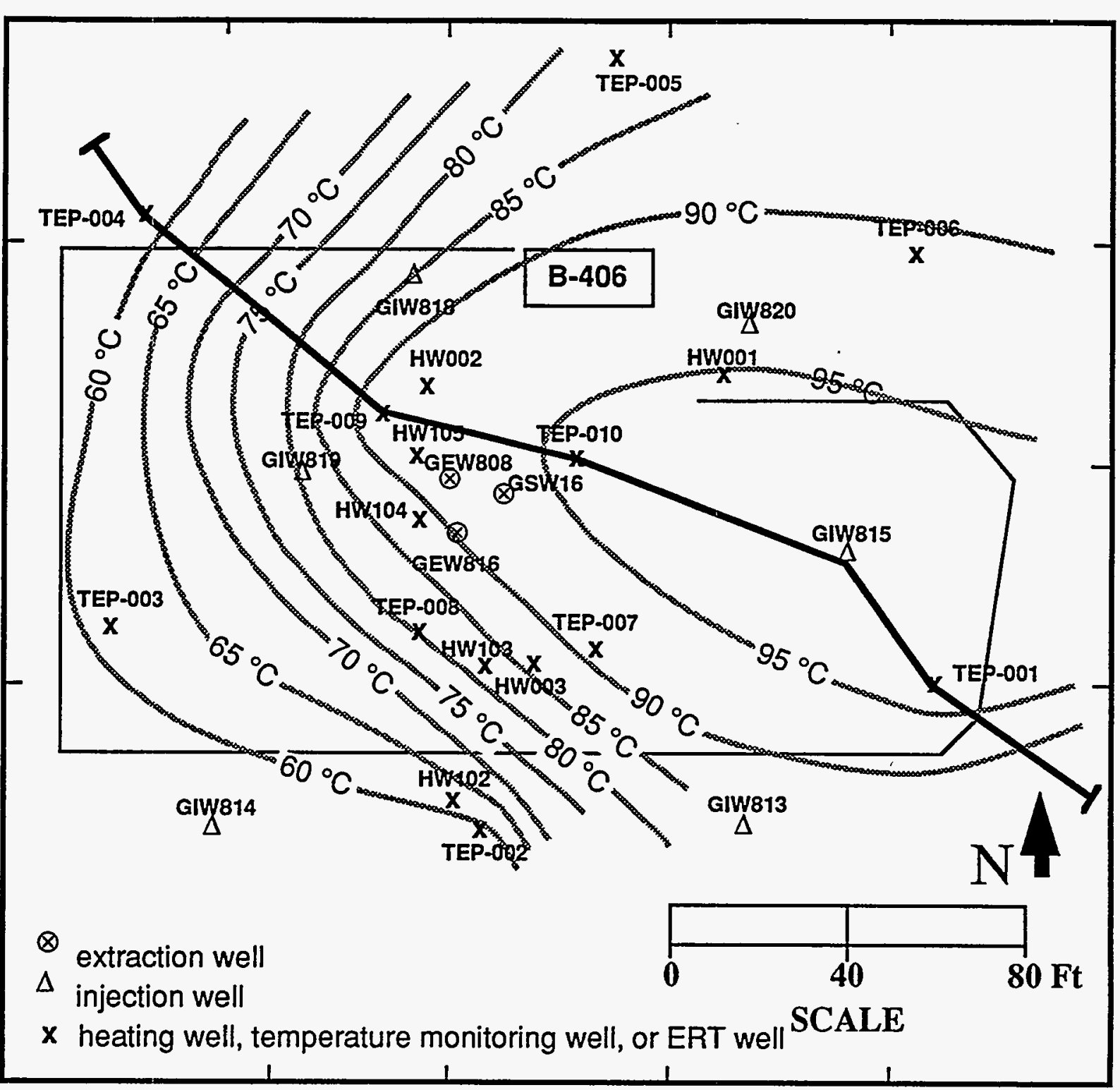

Figure 5. Plan view of temperature distribution in the upper steam zone (80-ft or $24.4-\mathrm{m}$ depth) at the beginning of ARV operations on October 5,1993 . Well symbols are the same as those used in Figure 1. This is the zone most strongly heated by steam injection during the DUSDP. The line of section shows the location of the cross section shown in Figure 6. 


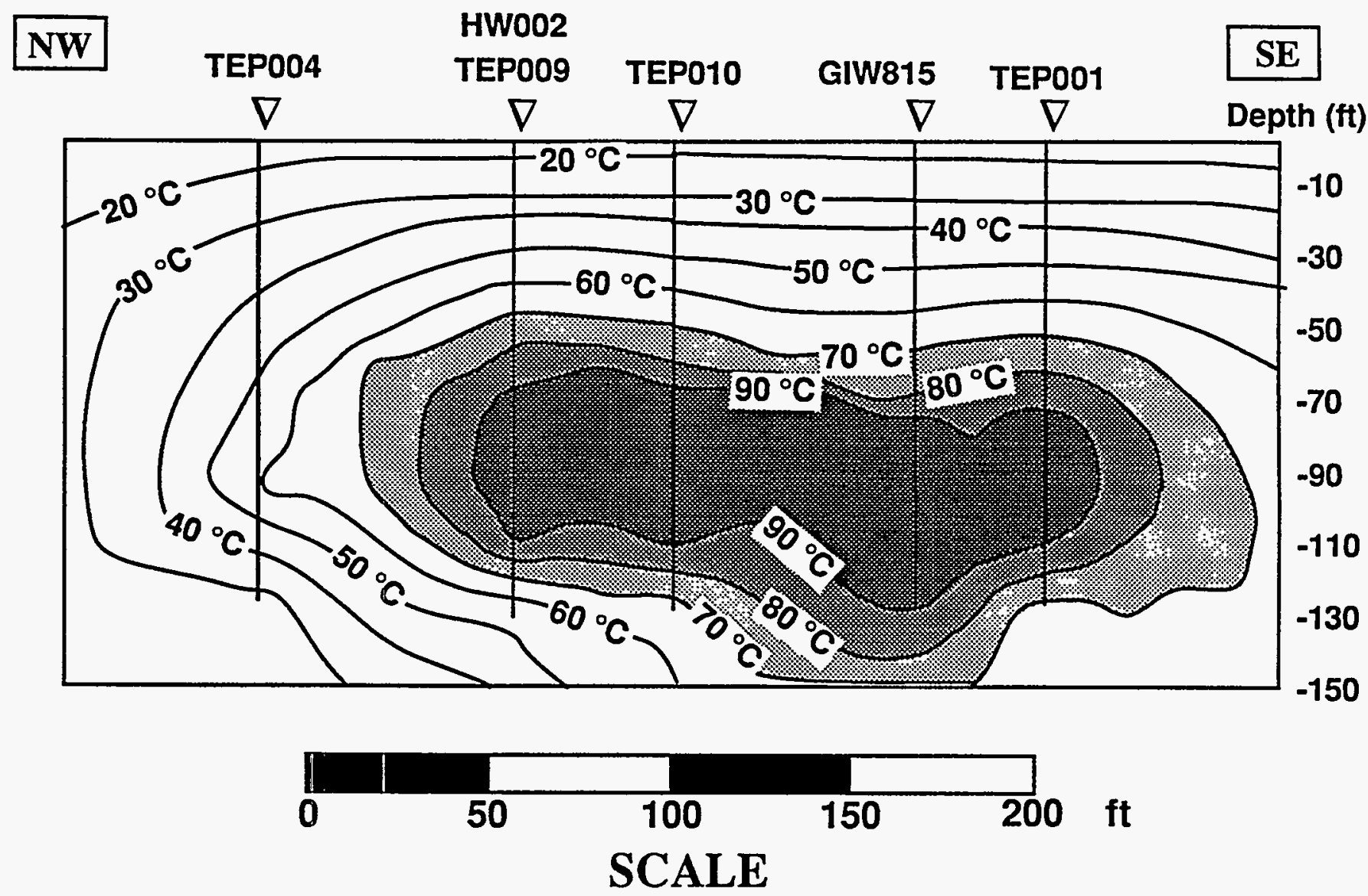

Figure 6. Cross section (see Fig. 5 for section line) showing temperature distribution at the TFF site at the beginning of October 1993. Well locations with data control points are indicated. Note the zone of high temperatures at the 70- to 130-ft depth (the location of the upper and lower steam zones and the clay aquitard) affected by steam during the DUSDP. 


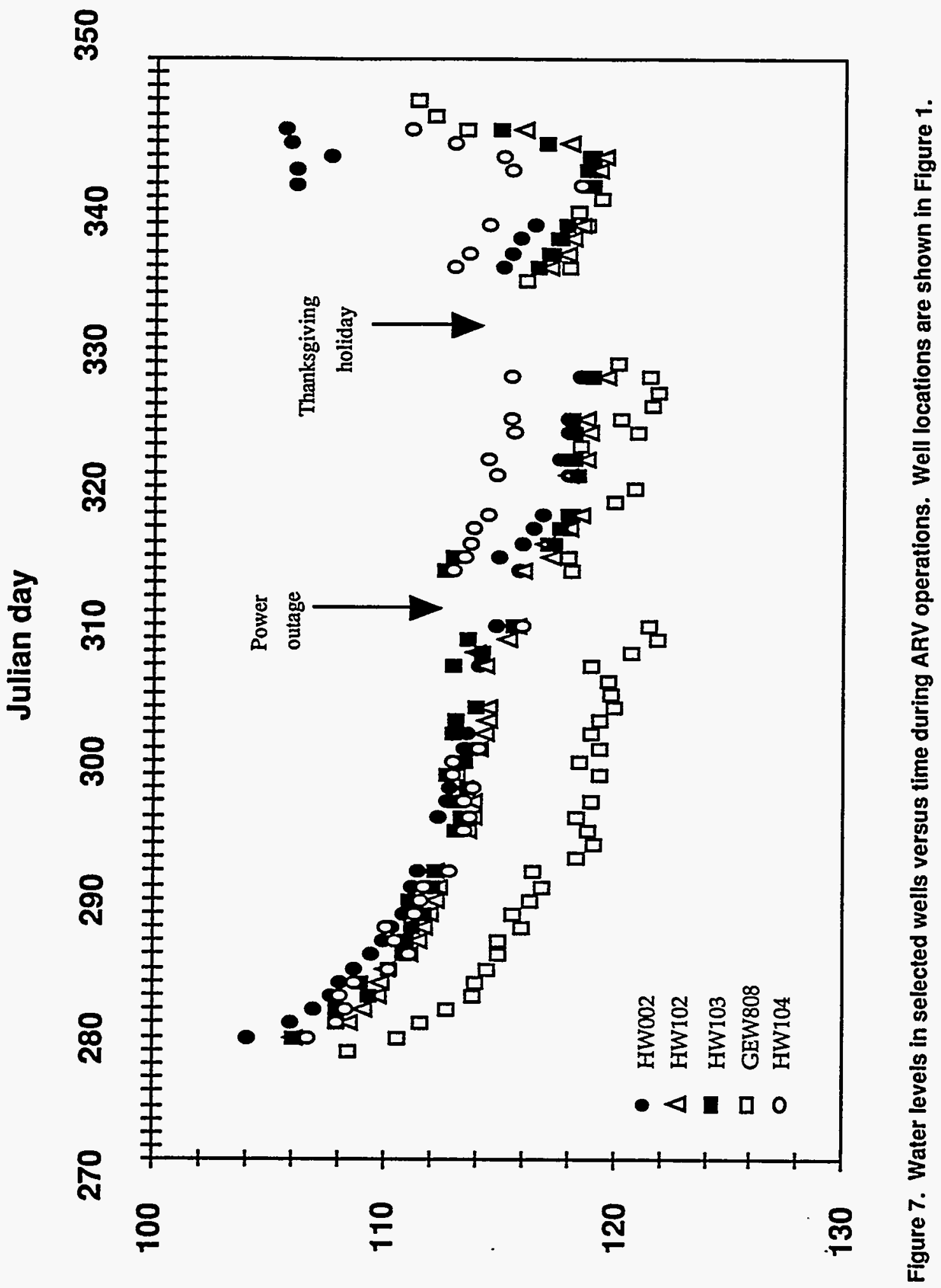

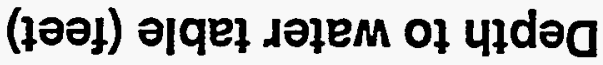




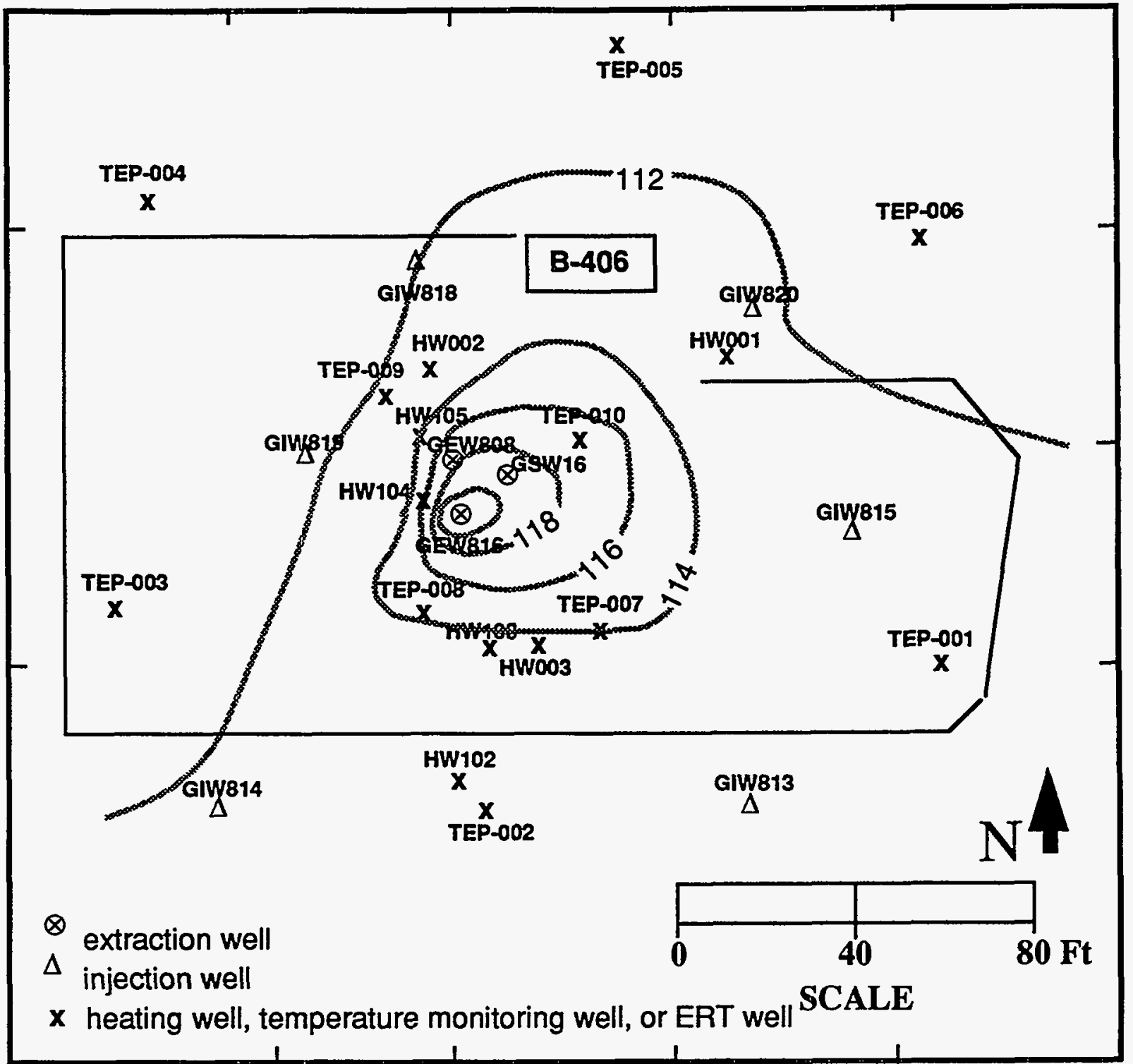

Figure 8. Plan view of the water table at the TFF site as measured on October 25, 1993, after 21 days of continuous pumping of ground water at about $50 \mathrm{gal} / \mathrm{min}$ from the central extraction wells (located at the center of the cone of depression). Contours are in feet below the surface. The background water level before pumping began was about 102 to $104 \mathrm{ft}$ deep. 
However, as stated above, the water levels are more indicative of head in the lower aquifer and do not imply significant drainage of the clay zone.

Pumping water draws in a significant volume of water from outside the area that had been affected by steam and would therefore be expected to cool the formation. This has in fact been observed, as seen in Figure 9, which shows the difference in temperature of the lower steam zone (130-ft or 39.6-m depth) between October 4, before 24-h pumping began, and October 28, after $23 \mathrm{~d}$ of continuous pumping. The greatest amount of cooling occurs to the west of the central extraction wells, where only about $2^{\circ} \mathrm{C}$ of cooling has occurred. Figure 9 shows that cooler water is obviously flowing into the TFF area from the south along a relatively narrow zone that is probably controlled by the local permeability. We also note that these dramatic cooling effects are not seen at other depths below TFF; this effect is confined to the lower steam zone aquifer, the zone that is being pumped. When Figure 9 is compared with Figure 10, which shows differences in temperatures between September 15 and October 4 (before we started continuous pumping of ground water), one can see the dramatic effect of ground water withdrawal on the subsurface temperatures. 


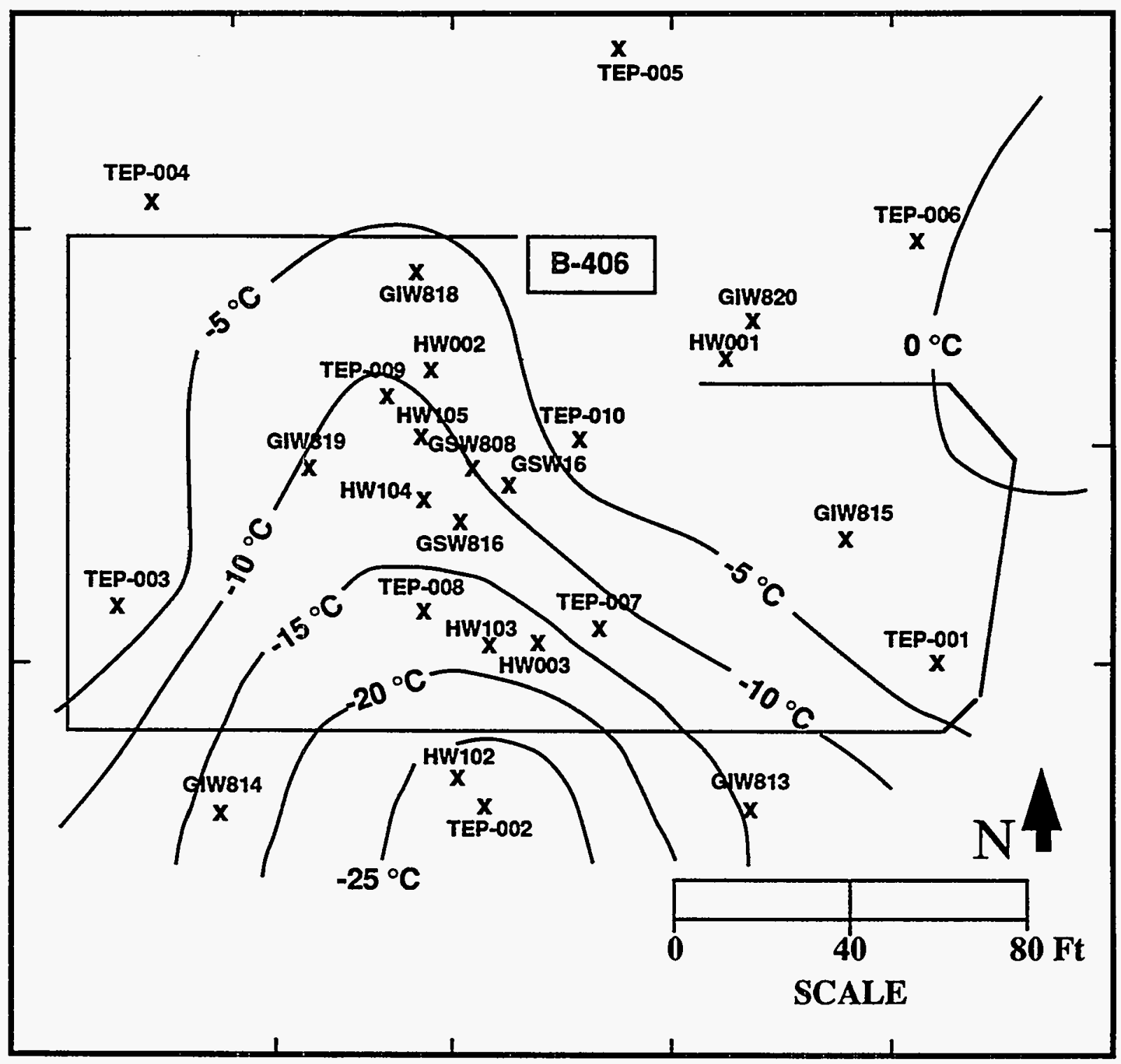

Figure 9. Plan view at the TFF site of the difference between October 4, 1993, and October 28, 1993, in the temperature at the 130-ft (39.6-m) depth, the depth of the lower steam zone. Temperature differences in degrees Celsius. Note the large zone of lowered temperatures due to the influx, within the lower steam zone aquifer, of cold water from the southern boundary of the site. 


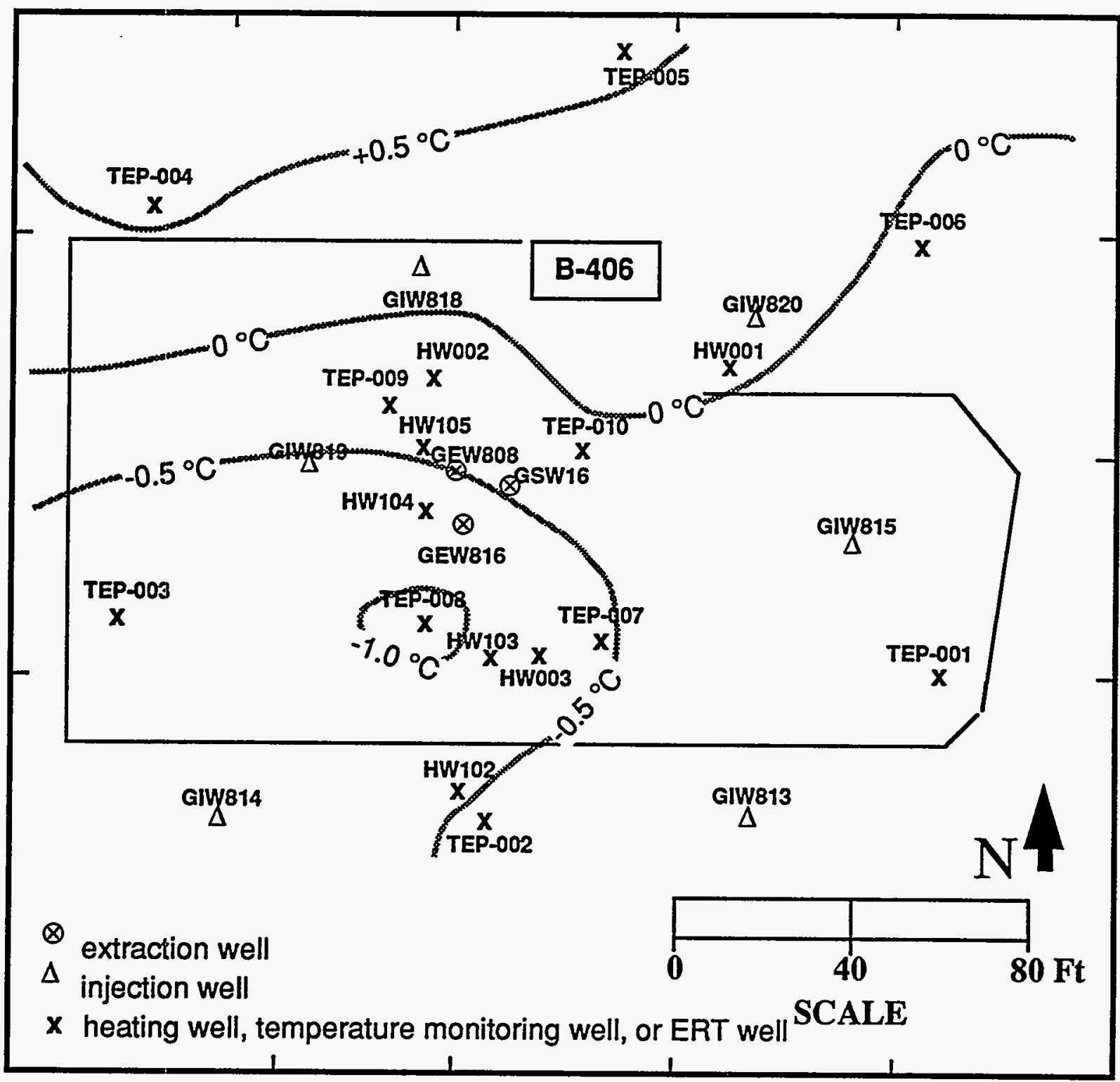

Figure 10. View similar to Figure 9, except that the difference in temperatures is between September 15, 1993, and October 4, 1993, when 24-h pumping of ground water was not taking place. Note the lack of perturbation of subsurface temperatures in the lower steam zone. 


\section{Air Sparging}

\section{Introduction and General Description}

In the context of ground water remediation, air sparging refers to the process of injecting air into the saturated zone while carrying out vapor extraction in the vadose zone. As air passes through the saturated sediments, free product and adsorbed and absorbed contaminants will evaporate and be carried away with the injected air. The injected air is then recovered by the vapor extraction system. The reasons for carrying out air sparging were discussed in the Introduction: the procedure is relatively cheap and easy to implement; high subsurface temperatures still available at the TFF site would enhance the volatilization of hydrocarbons; and the injected air would enhance microbial action and biodegradation of hydrocarbons. Because of the last point, we did not have much concern about the possibility that some of the injected air would not be recovered, but there remained a concern that some contaminant could be moved outward away from the central extraction well area. For this reason, it was important to be able to track the movement of the injected air and quantify how much of it was recovered.

In concept, it is a simple process to add air sparging onto an existing vapor extraction system. All that is needed is an air compressor, as a source of high pressure air, and wells with suitable screened intervals for injection and extraction. In practice, however, it is often difficult to determine just where the injected air goes and what effect it really has on the recovery of VOCs. In addition to buoyancy, the pattern of air flow in the subsurface will be controlled by the subsurface permeability distribution and geometry of the matrix material (e.g., a dipping surface of a high/low permeability contact may control the direction of flow and where the injected air will reach the vadose zone).

Because of the above concerns, we chose to carry out two small air sparging applications to test our ability to track the fate of the injected air and to determine what effect air sparging can have on recovery of hydrocarbons in a regime of elevated subsurface temperatures and with the water table lowered to expose a saturated aquitard. Electrical resistance tomography (ERT) wells were in place at the TFF site, so we decided to use LLNL capabilities in this area to see how well we could track the movement of the injected air. In addition, we were fortunate to be able to draw on the expertise and experience of personnel of the LLNL Nuclear Chemistry Division, who have been using noble gas isotope trace gases to monitor underground nuclear tests for many years. By injecting gas of a known composition and concentration along with the injected air and monitoring the tracer gases, we can determine how much of the recovered vapor at a given location is coming from the injected air, what the input flow rate is, and, with frequent sampling, how long it takes for the injected air to reach a sample location. In addition, we planned to take frequent samples of vapor at the injection and extraction wells for hydrocarbon chemistry analysis so that we could monitor the effect of sparging on hydrocarbon recovery. Because of the relatively rapid movement of air compared to water in the subsurface, all of the analyses of chemistry and trace gases had to be carried out with as short a turnaround as possible so that we could adequately determine what was happening and adjust the operations accordingly. For this reason, the fact that all analyses were done in-house, by LLNL personnel, was a great benefit. 
In addition to sampling vapor for trace gases, we also took water samples for trace gas analysis from a ring of wells surrounding the central TFF area about 2 weeks after the completion of the sparging. Different trace gas compositions (the percentages and isotopes of krypton and xenon are given below) were used for each sparging configuration. Because of these differences, we would be able to separate the effects of each application and be able to tell how much of the injected air was incorporated into the ground water and detected outside of the area where injection occurred. The water samples have not yet been analyzed as of the writing of this report, so we are unable to report on those results at this time.

Another available tool that we took advantage of at LLNL is computer modeling. A generalpurpose finite-difference fluid flow code, called NUFT, was developed by J. Nitao of the Earth Sciences Division at LLNL. This code is designed to simulate coupled fluid flow and heat transfer problems in three dimensions. It can model vapor, water, and free-product flow in both the saturated and vadose zone. The calculations can also simulate electrical heating from downhole electrodes and incorporated contaminant chemistry for comparing the relative effects of steam heating versus electrical heating. For this project, we utilized NUFT to simulate relatively simple aspects of air sparging, steam injection, and electrical heating. These simulations, which are described in Appendix 5, proved to be very helpful for providing insight into the various physical processes and helping us understand what happens during various types of remediation.

At the TFF site, the character of well completions, as well as other factors, limits our choices for injection/extraction geometry. Our choices of wells suitable for injection of air and vapor extraction are limited to the three central extraction wells and the ring of six steam injection wells used during the DUSDP. The desire to carry out ERT imaging during air injection limited us to the use of combinations of wells optimally located with respect to existing ERT image planes. The central vapor extraction wells, which are also used to pump ground water (see Section II), are screened over large intervals in both the vadose and saturated zones and thus cannot be used to inject air exclusively into the saturated zone. At the time the sparging took place, water levels in the extraction and injection wells had been considerably lowered due to the 24-h pumping operations described above; in some cases, water levels in the injection wells were below the top of the screened interval in the lower steam zone.

An additional important factor in this application is the geometry of the base of the clay aquitard, which lies between the upper and lower steam zones. We suspected that this clay zone could impede upward movement of air; but, because of heterogeneities, we were not sure how effective a barrier it would be. Beneath the central part of the TFF site, the clay layer generally slopes from the southeast toward the northwest (Fig. 11) so that injected air, if trapped beneath the clay zone, could be expected to move toward the east and southeast. Limitations in the well and ERT configurations dictated the choice of injection and extraction wells for the sparging. We could do nothing about the configuration of the clay zone.

For the first sparging application, air was injected into the lower steam zone in injection well GIW820 while vapor extraction was carried out at the central extraction wells (GEW808, GEW816, and GSW16). Vapor samples, for analysis of both hydrocarbon chemistry and tracer gases, were taken at the injection well and at the outlet of the extraction wells. During and following cessation of air injection at GIW820, we also monitored the vapor concentration at injection well GIW815. The base of the aquitard slopes downward from GIW820 to GIW815 (Fig. 11). In this case, air injection rates averaged slightly less than one-half that of the 


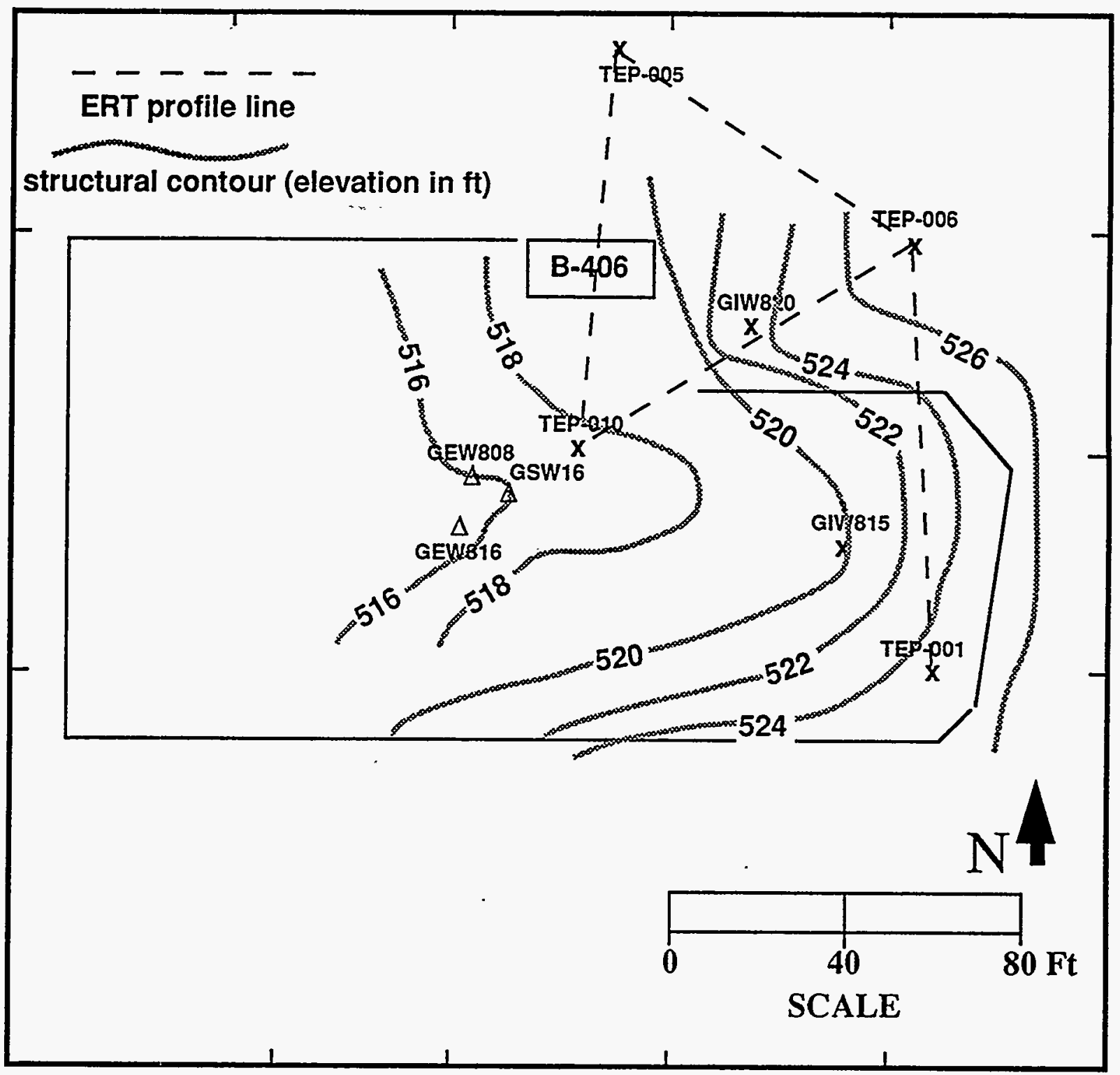

Figure 11. Location of wells and ERT profile lines used in the air sparging applications. Also shown are structure contours (elevation, in feet above mean sea level) of the base of the clay aquitard. 
extraction rates. Within $5 \mathrm{~h}$ of the start of injection, we confirmed that most of the injected air was moving upslope to the east of wells GIW815 and GIW820, with only a very small part of the injected air being recovered by the extraction wells. Air injection was stopped after $7 \mathrm{~h}$. Details of this application are given below.

The second sparging application was carried out one week after the first one, after vapor extraction conditions had been allowed to return to a stable level for reference. In this case, we injected air into well GIW815 and extracted vapor from well GIW820. Because the base of the aquitard at GIW820 is slightly upslope from where it is at GIW815, we presumed the air would have less of a geometric barrier to overcome and more of the injected air would be recovered. The injection rate in this case was about 0.8 times the extraction rate, and we eventually were able to recover about $45 \%$ of the injected air. A check of the water level in GIW815 after the air injection was stopped indicated that most of the air was injected in the vadose zone and not in the saturated zone as we had planned. Details are given below.

\section{Air Sparging I}

The first air sparging application took place November 16, 1993. An air compressor (modified with a conditioner to remove any oil or fuel from the output air) was attached to the steam injection manifold with connections to well GIW820. On November 8, vapor extraction was switched to the central extraction wells (GEW808, GEW816, GSW16) so that we could achieve a reliable background for reference on hydrocarbon removal from these wells prior to starting air sparging. The locations of the injection well, extraction wells, monitoring well GIW815 (see below), and the ERT profile planes are shown in Figure 11. Injected air flow rates were monitored by using calibrated measurements of absolute pressure and differential pressure, which gave us estimates of flow rates accurate to about $20 \%$. The flow rate can also be monitored by sampling the composition of tracer gas in the air at the well head, as discussed below. Air was injected into the lower screened interval (112- to 132-ft depth) of well GIW820 beginning at 8:40 a.m. The initial pressure at the inlet steam manifold was 9 psi, gradually decreasing to a value of $6.6 \mathrm{psi}$ when the compressor was shut off $7 \mathrm{~h}$ later. This initial injection pressure confirms that most of the air was being injected in the saturated zone. Injection rates, monitored at the input to the steam manifold every $30 \mathrm{~min}$, were very constant at about $44 \mathrm{scfm}$. Extraction rates during the time of air injection were measured every $2 \mathrm{~h}$ at the ICE and ranged from 101 to $106 \mathrm{scfm}$.

For this sparging application, the trace gas composition was: $22 \mathrm{Ne}, 0.68 \% ; 78 \mathrm{Kr}, 0.002 \%$; $124 \mathrm{Xe}, 0.000 \%$; and air, $99.31 \%$. The trace gases were injected at a rate of $1.0 \mathrm{~L}(\mathrm{STP}) / \mathrm{min}$. Knowing the rate at which the trace gas was injected into the input air, we can determine the flow rate of the injected air via Figure 12. Measured values of the $22 \mathrm{Ne} / 20 \mathrm{Ne}$ ratio at the input of the injection well ranged from 0.364 to 0.370 , which corresponds to flow rates of 53 to 55 scfm. This value is about 15 to $20 \%$ higher than that estimated from the pressure gauges (see above), but well within the uncertainty of the pressure gauge measurements. The value of 53 to $55 \mathrm{scfm}$ is probably more accurate.

Our intuitive concept of the air sparging process is that air injected into the lower steam zone in GIW820 would move buoyantly upward through the permeable sandy unit until it reaches the low-permeability clay zone that separates the upper and lower steam zones. Upon reaching this 


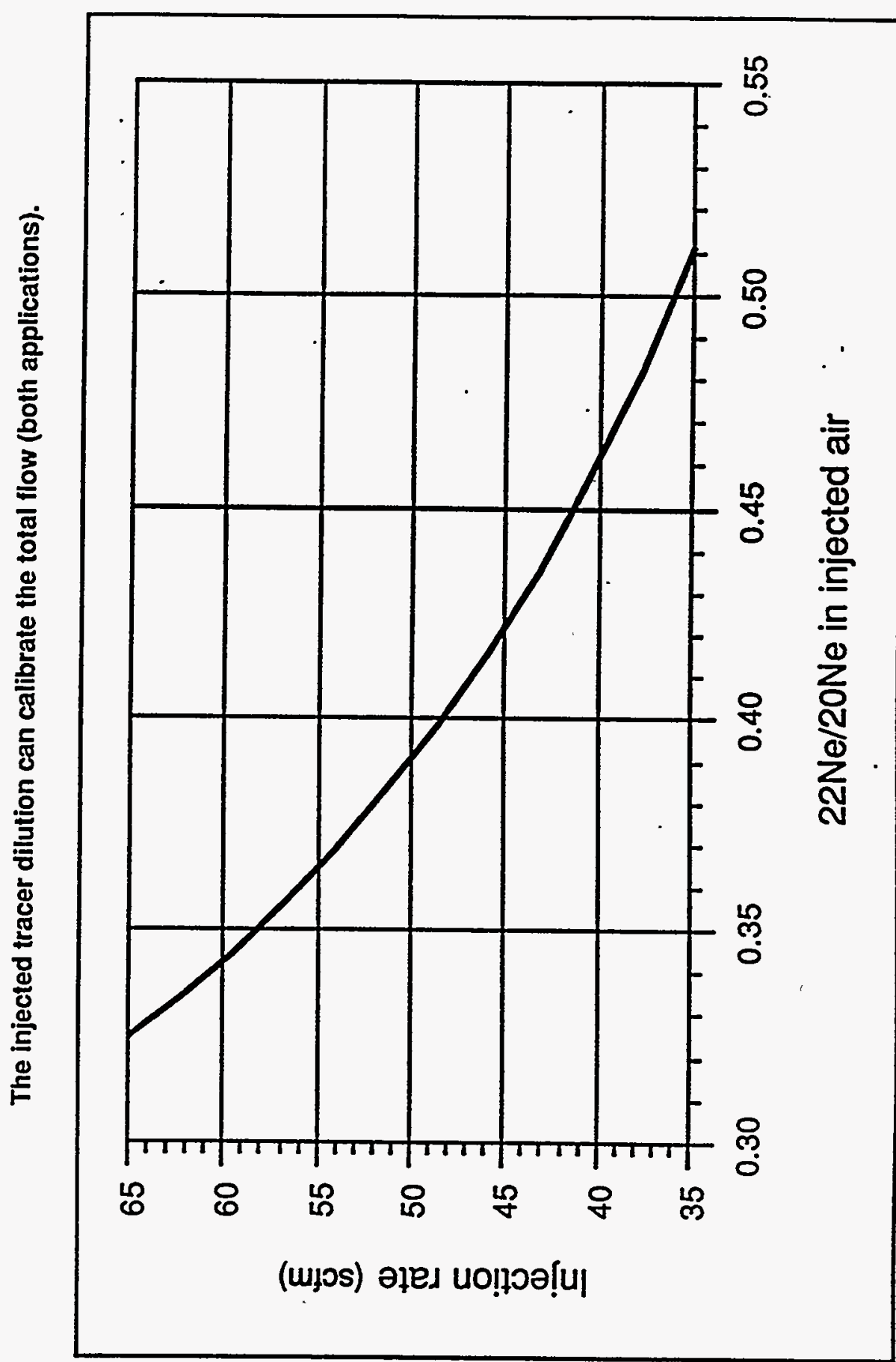

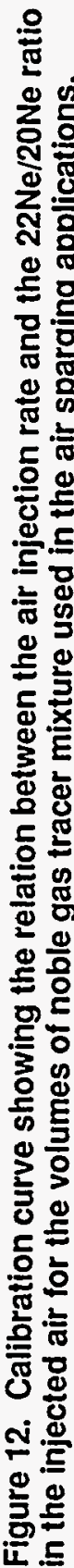


barrier to upward movement, the air would move laterally, under the control of the slope on the base of the lower clay zone, until it reached some vertical discontinuity, such as a fracture or hydraulically conductive zone. This conductive pathway would allow the air to move upward into the vadose zone where it would come under the influence of the vapor extraction system. Using this concept and a cross section of the area between GIW820 and the extraction wells (Fig. 13), it appeared that the most likely path for the air was away (up, along the base of the confining zone) from the extraction wells. The gradient of the base of the clay zone is smaller to the south, so we decided to use GIW815 (Fig. 11) as a monitoring well to determine when and if injected air might pass in that direction.

These conceptual ideas were tested with a computer simulation, described in Appendix 5. The simulation suggested that most of the air would move toward the south and east, away from the extraction wells, and that none of the injected air would reach the extraction wells in the saturated zone. Without detailed knowledge of the subsurface heterogeneity, we could not be sure if possible "easy" paths to the vadose zone would affect the results. In addition, because the extraction wells are screened in both the vadose and saturated zones, we had no way to discriminate where the sampled air was coming from. The simulation also showed that the vapor extraction rate has little to no influence on the movement of air injected in the saturated zone. In order to have influence on the injected air comparable in any way to the buoyancy forces, we would have to pump ground water at the extraction wells at a volumetric rate greater than that of the air injection rate. This turned out to be a rate far in excess of the $50 \mathrm{gal} / \mathrm{min}$ rate of pumping the wells were capable of.

The trace gases were sampled approximately hourly at (1) the input to the injection well, (2) the input to the ICE (representing the composite of the vapor being extracted from the central extraction wells), (3) the lower steam zone interval of GIW815 (GIW815L) (112- to 132-ft depth), and (4) the upper steam zone interval of GIW815 (GIW815U) (77- to 97-ft depth). Vapor samples for noble gas tracers were taken by attaching an evacuated one-liter steel cylinder to the sampling port and opening the sampling port valve for $5 \mathrm{~min}$. Depending on the flow rate of the input air, the $22 \mathrm{Ne} / 20 \mathrm{Ne}$ ratio of the injected air varied from 0.364 to 0.370 . Air recovered at various sampling points would have a $22 \mathrm{Ne} / 20 \mathrm{Ne}$ ratio of 0.102 , the background air value measured before the start of sparging, if none of the injected air was present. Values for the $22 \mathrm{Ne} / 20 \mathrm{Ne}$ ratio above 0.102 meant that a portion of the injected air was present in the sample. The fraction of injected air in the sample can be calculated from:

$$
\mathrm{F}=\left[(22 \mathrm{Ne} / 20 \mathrm{Ne})_{\text {meas }}-(22 \mathrm{Ne} / 20 \mathrm{Ne})_{\text {air }}\right] /\left[(22 \mathrm{Ne} / 20 \mathrm{Ne})_{\text {inject }}-(22 \mathrm{Ne} / 20 \mathrm{Ne}) \text { air }\right]
$$

where the subscripts refer to the Ne ratio measured in the sample, in background air, and in injected air, respectively. $F$ is the fraction due to the tracer.

Table 2 shows the variation in the $22 \mathrm{Ne} / 20 \mathrm{Ne}$ ratio versus time at the four sampling points. The flow rate at the injection well is determined from Figure 12. The percent injected air in the sample shown in Table 2 is the fractional value F times 100\%. Note that GIW815 in this case was only being used as a monitoring well, and vapor was not being extracted from either the upper or lower steam interval. A vacuum hose was connected to each sampling port in GIW815 for 5 min each time before sampling in order to purge gas that accumulated between sampling intervals. Because there was no continuous flow in GIW815, these trace gas values can only be 


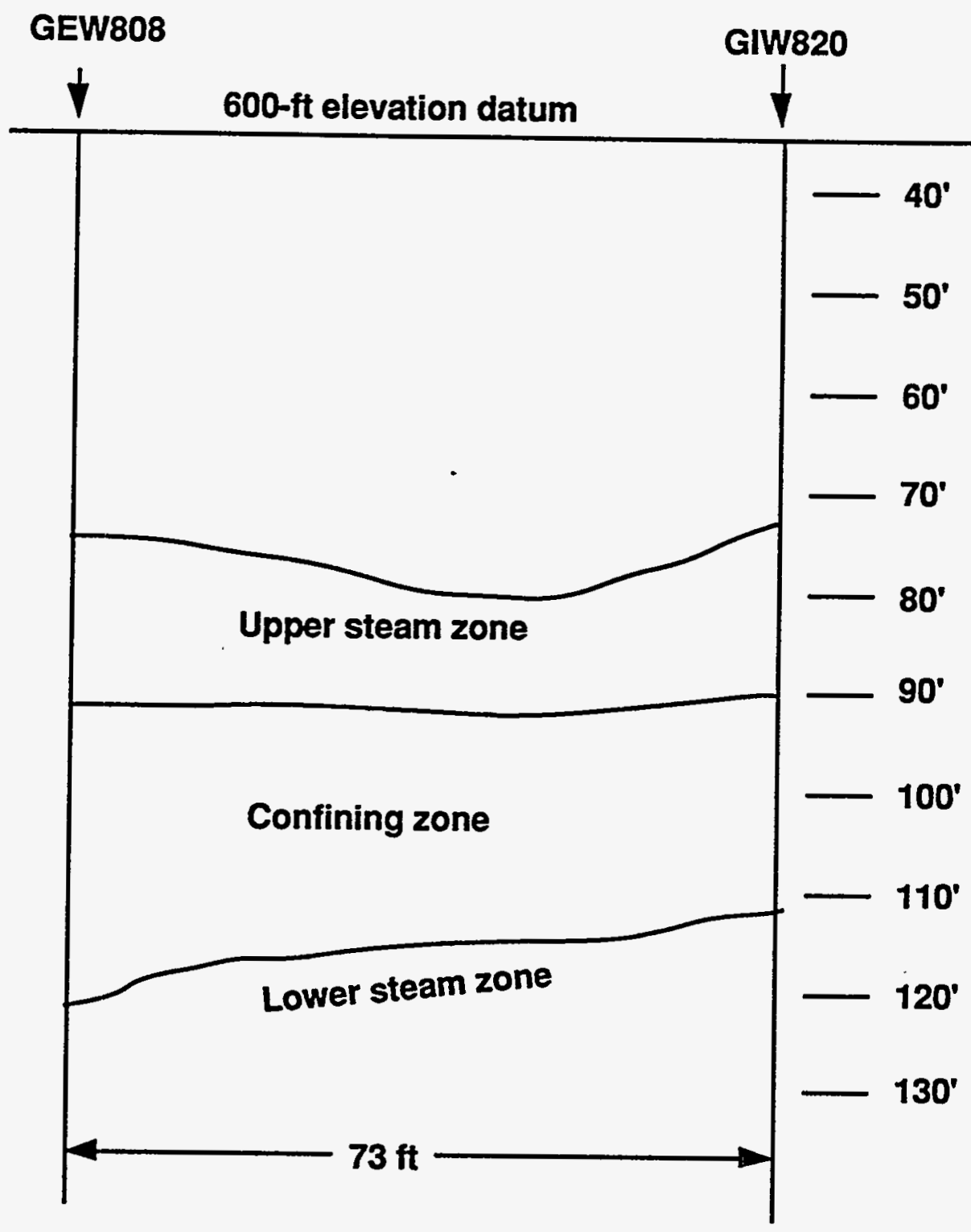

Figure 13. Cross section showing the simplified geology between well GIW820 and GEW808, one of the central extraction wells. Flow of air injected into the lower steam zone of GIW820 will be controlled by slope of the base of the confining zone (clay aquitard). 
Table 2. Tracer gas analysis from the first sparging application.

\begin{tabular}{|c|c|c|c|c|c|}
\hline Wells & Date & Time & $22 \mathrm{Ne} / 20 \mathrm{Ne}$ & $\begin{array}{c}\text { Flow rate } \\
\text { (scfm) }\end{array}$ & \\
\hline \multirow[t]{5}{*}{ Injection Well } & $11 / 16 / 93$ & $8: 52$ & 0.364 & 55.0 & \\
\hline & $11 / 16 / 93$ & $9: 15$ & 0.368 & 53.5 & \\
\hline & $11 / 16 / 93$ & $10: 17$ & 0.370 & 53.5 & \\
\hline & $11 / 16 / 93$ & $15: 23$ & 0.365 & 54.0 & \\
\hline & Date & Time & $\begin{array}{c}\text { Elapsed time } \\
\text { (h) }\end{array}$ & $22 \mathrm{Ne} / 20 \mathrm{Ne}$ & $\begin{array}{c}\% \text { injected } \\
\text { air }\end{array}$ \\
\hline \multirow[t]{12}{*}{ ICE-in } & $11 / 16 / 93$ & $10: 41$ & 2.0 & 0.102 & 0.0 \\
\hline & $11 / 16 / 93$ & $11: 50$ & 3.2 & 0.102 & 0.0 \\
\hline & $11 / 16 / 93$ & $12: 34$ & 3.8 & 0.125 & 8.6 \\
\hline & $11 / 16 / 93$ & $13: 25$ & 4.8 & 0.102 & 0.0 \\
\hline & $11 / 16 / 93$ & $13: 57$ & 5.3 & 0.103 & 0.4 \\
\hline & $11 / 16 / 93$ & $14: 30$ & 5.8 & 0.103 & 0.4 \\
\hline & $11 / 16 / 93$ & $14: 59$ & 6.3 & 0.106 & 1.5 \\
\hline & $11 / 16 / 93$ & $15: 30$ & 6.8 & 0.103 & 0.4 \\
\hline & $11 / 16 / 93$ & $15: 55$ & 7.3 & 0.110 & 3.0 \\
\hline & $\mathbb{1 1 / 1 6 / 9 3}$ & $18: 00$ & 9.3 & 0.103 & 0.4 \\
\hline & $11 / 16 / 93$ & $20: 00$ & 11.3 & 0.103 & 0.4 \\
\hline & $11 / 17 / 93$ & $8: 35$ & 24.0 & 0.108 & 2.2 \\
\hline \multirow[t]{9}{*}{ GIW815L } & $11 / 16 / 93$ & $9: 35$ & 1.9 & 0.102 & 0.0 \\
\hline & $11 / 16 / 93$ & $10: 00$ & 2.3 & 0.104 & 0.8 \\
\hline & $11 / 16 / 93$ & $10: 48$ & 3.1 & 0.330 & 86.7 \\
\hline & $11 / 16 / 93$ & 11:18 & 3.6 & 0.361 & 98.5 \\
\hline & $11 / 16 / 93$ & $12: 05$ & 4.4 & 0.367 & 100.8 \\
\hline & $11 / 16 / 93$ & $13: 00$ & 5.3 & 0.367 & 100.8 \\
\hline & $11 / 16 / 93$ & $14: 00$ & 6.3 & 0.365 & 100.0 \\
\hline & $11 / 16 / 93$ & $15: 00$ & 7.3 & 0.248 & 55.5 \\
\hline & $11 / 16 / 93$ & $15: 56$ & 8.3 & 0.140 & 14.4 \\
\hline \multirow[t]{6}{*}{ GIW815U } & $11 / 16 / 93$ & $10: 26$ & 1.8 & 0.128 & 9.9 \\
\hline & $11 / 16 / 93$ & $11: 30$ & 2.8 & 0.116 & 5.3 \\
\hline & $11 / 16 / 93$ & $12: 43$ & 4.1 & 0.116 & 5.3 \\
\hline & $11 / 16 / 93$ & $13: 43$ & 5.1 & 0.114 & 4.6 \\
\hline & $11 / 16 / 93$ & $14: 45$ & 6.1 & 0.111 & 3.4 \\
\hline & $11 / 16 / 93$ & $15: 45$ & 7.1 & 0.112 & 3.8 \\
\hline
\end{tabular}


used to get some indication of the relative amounts of injected air passing in that direction. Table 2 shows that the first trace of injected air was seen in the lower steam interval at GIW815 in the 10:00 a.m. sample. Thus, the total travel time for the air in the saturated zone between GIW820 and GIW815 (about $70 \mathrm{ft}$ distance) was about $2.3 \mathrm{~h}$. The amount of injected air detected in the lower interval of GIW 815 reached $100 \%$ by $4.4 \mathrm{~h}$ into the sparging, with values dropping off to $55.5 \%$ just before air injection was stopped. This suggests that the flow path somehow became altered after about $6 \mathrm{~h}$.

Table 2 shows that some of the injected air was also getting into the vadose zone, as shown by the tracer values obtained for the upper steam interval in GIW815. The initial value was the highest, with the percent of injected air decreasing gradually to about $4 \%$ after $7 \mathrm{~h}$. The fact that tracer air reached the upper interval of GIW815 before it reached the lower interval suggests that the input air was getting into the vadose zone very quickly. Values of samples from the input to the ICE show rather erratic behavior. These samples probably represent air coming from the vadose zone. The first sample of injected air was detected after about $3.8 \mathrm{~h}$, but subsequent samples showed very low to no injected air component for the next $2 \mathrm{~h}$. A later peak at the ICE-in sample port of $3.0 \%$ injected air occurred after $7.3 \mathrm{~h}$. A trace of injected air was still seen in the ICE-in the next morning, $24 \mathrm{~h}$ after injection began and more than $16 \mathrm{~h}$ after air injection ceased.

We also took samples of vapor for hydrocarbon chemistry analysis at the input to the ICE and in the lower steam interval of GIW815. These samples were taken in Tedlar bags using a vacuum pump and desiccator jar to inflate the bags (see Appendix 4 for details). Sampling for hydrocarbon chemistry was done at intervals similar to those for the trace gases. Table 3 shows the measured values of total petroleum hydrocarbons (TPH) (analysis up through the $\mathrm{C}_{12}$ fraction), in ppmv. Because the flow rates from the input of the ICE are known, the mass extraction rate can be calculated, as shown in Table 3. The mass removal rate steadily increases until $6.3 \mathrm{~h}$ and then rapidly decreases. The TPH analyses for the samples from GIW815 cannot be interpreted in terms of mass removal because there is no flow in the well, but the data show a dramatic increase in the amount of hydrocarbons present after the first $2 \mathrm{~h}$ when the tracer gas data indicates that the vapor consists of $100 \%$ injected air. This suggests that the injected air is picking up a significant amount of hydrocarbons along its path in the saturated zone between GIW820 and GIW815.

The ERT difference images taken during the sparging are shown in Figure 14. Each image in Figure 14 shows the difference, between measurements taken at different times, in subsurface electrical resistivity over a cross section between two wells. The section TEP10-TEP6 (Fig. 11) runs almost parallel to the direction between GIW820 and the extraction wells. The section TEP10-TEP5 runs almost due north-south and at about $60^{\circ}$ to the TEP10-TEP6 section. Section TEP5-TEP6 runs northwest-southeast and completes the triangle of the three wells. The lower two images show differences along section TEP10-TEP6 for measurements taken between 9:30 a.m. and 10:22 a.m. (left), and images taken between 9:30 a.m. and 12:57 p.m. (right), while air was being injected into GIW820 between depths of 112 to $132 \mathrm{ft}$. There is not much to be seen after only $1 \mathrm{~h}$ (lower left image), but after $3 \mathrm{~h}$ of air injection, resistivity increases about $0.5 \mathrm{ohm}-\mathrm{m}$ (lower right image). The zone of increased resistivity is centered on the area of the lower steam zone interval of GIW820 and extends slightly upward and toward the northeast (to the right in the cross section). This agrees with our intuition and modeling that indicated that the 
Table 3. Hydrocarbon chemistry and cumulative mass removal during the first sparging application.

\begin{tabular}{|c|c|c|c|c|c|c|c|}
\hline & $\begin{array}{c}\text { Elapsed } \\
\text { time } \\
\text { (h) }\end{array}$ & $\begin{array}{c}\text { Flow rate } \\
\text { (scfm) }\end{array}$ & $\begin{array}{c}\text { TPH } \\
(\mathrm{mg} / \mathrm{L})\end{array}$ & $\begin{array}{l}\text { Total mass } \\
\text { removal } \\
(\mathrm{g})\end{array}$ & $\begin{array}{c}\text { Mass } \\
\text { removal rate } \\
(\mathrm{g} / \mathrm{h})\end{array}$ & $\begin{array}{l}\text { Cumulative } \\
\text { mass removal } \\
\text { (g) }\end{array}$ & $\begin{array}{c}\text { Cumulative } \\
\text { mass removal } \\
\text { (gal) }\end{array}$ \\
\hline \multirow[t]{13}{*}{ ICE } & 1.0 & 101 & 0.7 & 120 & 120 & 120.12 & 0.04 \\
\hline & 1.8 & 101 & 4.7 & 672 & 806 & 792.19 & 0.26 \\
\hline & 2.8 & 102 & 5.7 & 988 & 988 & $1,779.96$ & 0.59 \\
\hline & 4.3 & 106 & 3.8 & 1,027 & 684 & $2,806.46$ & 0.94 \\
\hline & 5.3 & 106 & 5.4 & 972 & 972 & $3,778.94$ & 1.26 \\
\hline & 6.3 & 106 & 6.9 & 1,243 & 1,243 & $5,021.55$ & 1.67 \\
\hline & 7.3 & 106 & 2.9 & 522 & 522 & $5,543.80$ & 1.85 \\
\hline & 9.3 & 101 & 1.1 & 378 & 189 & $5,921.31$ & 1.97 \\
\hline & 11.3 & 101 & 2.1 & 721 & 360 & $6,642.00$ & 2.21 \\
\hline & 13.3 & 101 & 1.6 & 549 & 275 & $7,191.10$ & 2.40 \\
\hline & 16.3 & 101 & 1.9 & 978 & 326 & $8,169.19$ & 2.72 \\
\hline & 19.3 & 101 & 0 & 0 & o & 8,169.19 & 2.72 \\
\hline & 23.3 & 101 & 0.9 & 618 & 154 & $8,786.92$ & 2.93 \\
\hline \multirow[t]{9}{*}{ GIW815-L } & 1.0 & 5 & 0.01 & & & & \\
\hline & 1.3 & & & \multicolumn{4}{|c|}{ (1\% tracer recovered at GIW815-lower) } \\
\hline & 1.8 & 5 & 4.0 & & & & \\
\hline & 2.3 & & & \multicolumn{4}{|c|}{ (100\% tracer recovered at GIW815-lower) } \\
\hline & 3.0 & & & \multicolumn{4}{|c|}{ (6\% tracer recovered at GIW815-upper) } \\
\hline & 3.3 & 5 & 5.8 & & & & \\
\hline & 4.3 & 5 & 21.0 & & & & \\
\hline & 5.3 & 5 & 23.0 & & & & \\
\hline & 6.3 & 5 & 20.0 & & & & \\
\hline
\end{tabular}




\section{November 16 differences}
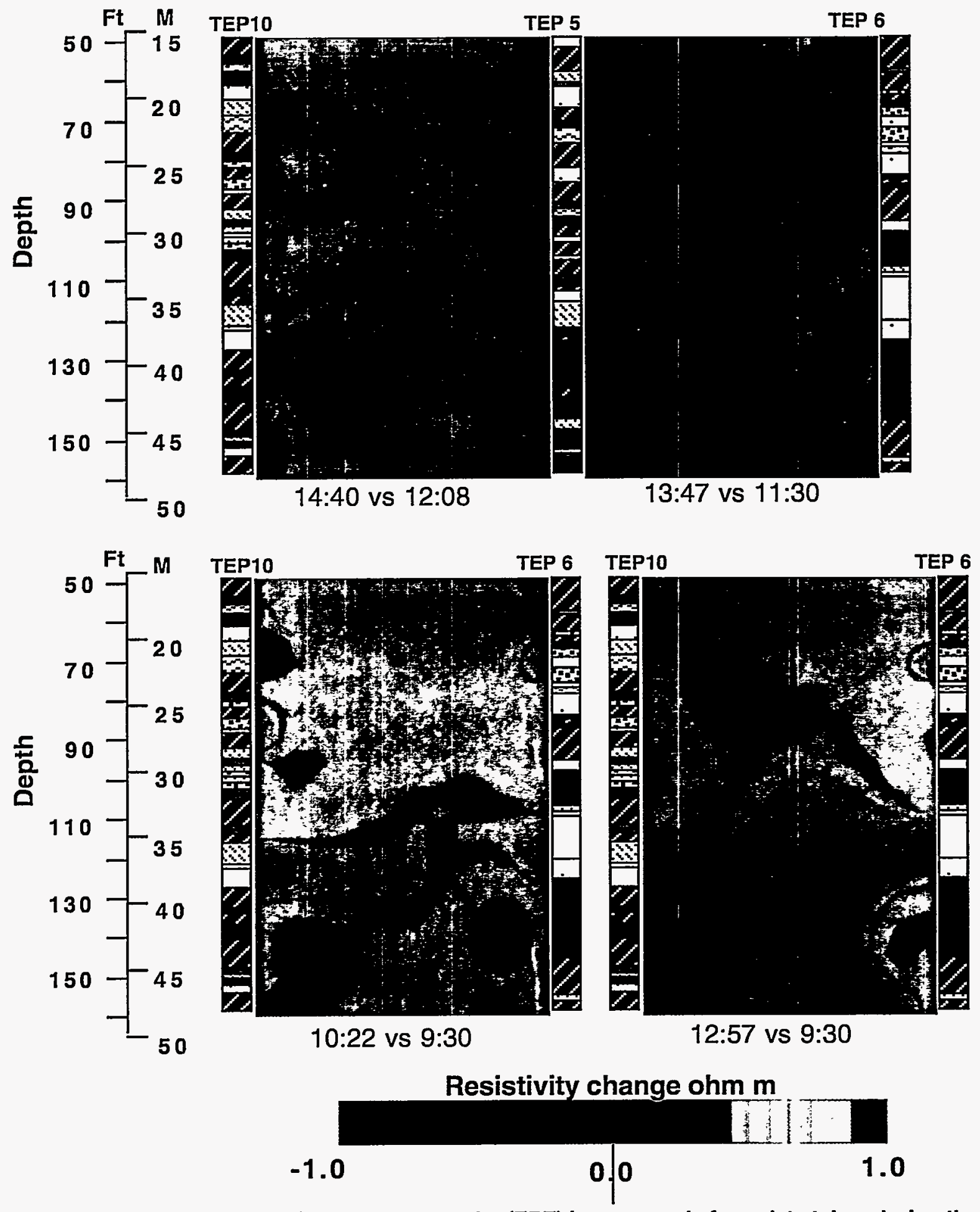

Figure 14. Electrical resistance tomography (ERT) images made from data taken during the first air sparging application. Refer to text for explanation of each part of the figure. 
injected air (which will tend to dry the formation and increase the resistivity) will move upward and to the east. The upper two images, TEP10-TEP5 (left) and TEP5-TEP6 (right), do not show any definitive changes due to air injection. The zone of slightly enhanced resistivity near TEP10 on the upper left image, which occurs in the saturated zone, is probably not due to the flow of injected air, because the tracer data suggest that only a small amount of injected air went toward the extraction wells and that air was probably in the vadose zone. The TEP10-TEP6 image on the lower right, however, suggests that ERT imagery may be useful for tracking injected air. If the difference image were done over a longer time interval, the assessment of usefulness could be more definitive.

\section{Air Sparging II}

The first air sparging application was terminated after $7.3 \mathrm{~h}$ of air injection because we had confirmed that most of the injected air was not being recovered by the extraction wells. Movement of the injected air was controlled by buoyancy and by the slope of the base of the confining clay layer. The first sparging also indicated that injected air easily reached monitor well GIW815, located about $70 \mathrm{ft}$ to the southeast of the injection well. For these reasons, we decided to use the lower steam interval of GIW815 as the injection well and GIW820 as the extraction well for the second sparging application. In order to establish stable background conditions, vacuum extraction was switched from the central extraction wells to GIW820 on November 18, continuing through the weekend. Because we were now extracting vapor from only one well instead of three, the extraction rate at the input to the ICE dropped from $101 \mathrm{scfm}$ to about $47 \mathrm{scfm}$.

Air injection started at 8:49 a.m. on November 22 with air injection into the lower steam interval of GIW815, and vapor extraction from both the upper and lower intervals of GIW820. The screened interval in the lower steam zone of GIW820 is in the saturated zone; thus, almost all of the flow in GIW820 was coming from the upper steam zone (located in the vadose zone). Initial air injection pressures at GIW815 were about one psi; this suggested that the lower steam zone of GIW815 had been desaturated and that we were injecting air into the vadose zone. After completion of the injection, the water level in GIW815 was tagged at a depth of $119 \mathrm{ft}$. This is $7 \mathrm{ft}$ below the top of the screened interval for the lower steam zone in the well, confirming that most of the air had probably been injected into the vadose zone. Initial injected air flow rates were estimated from the in-line gauges to be about $35 \mathrm{scfm}$, while analysis of the trace gas data suggested a somewhat higher rate of about $45 \mathrm{scfm}$.

We had originally intended for this sparging to run continuously for several days. However, the schedule for the start-up of the electrical heating operation required that initial testing of the electrical system had to begin on the evening of November 22, so we chose to shut down the air sparging operation between 3:30 p.m. and 9:00 p.m. on November 22 while vapor extraction was switched back to the central extraction wells. At 9:00 p.m., vapor extraction was switched back to GIW820 upper and lower and air injection continued overnight. At 10:00 a.m. the next day, we discovered that the air compressor had run out of fuel and had shut down some time between 9:00 a.m. and 10:00 a.m. The air compressor was refueled and restarted at 11:00 a.m., and air injection continued until 3:15 p.m. when we shut down sparging operations in order to prepare the TFF site for operational shutdown over the Thanksgiving holiday. The overall total time of air injection for this application was about $22.5 \mathrm{~h}$. 
The trace gas composition used was $22 \mathrm{Ne}, 0.68 \% ; 86 \mathrm{Kr}, 0.085 \%$; $136 \mathrm{Xe}, 0.004 \%$; and air, 99.23\%. Note that both the particular isotope and percentage of $\mathrm{Kr}$ and $\mathrm{Xe}$ are different from those of the first application. Again, the trace gas mixture was injected into the input air stream at a rate of $1.0 \mathrm{~L}$ (STP)/min. As of this date, only the Ne ratios have been analyzed. Measured values of the $22 \mathrm{Ne} / 20 \mathrm{Ne}$ ratio at the input to GIW815 (Table 4) ranged from 0.39 to 0.46 , giving (from Fig. 12) an air injection rate of 51 to $40 \mathrm{scfm}$; again, a slightly higher value than indicated by the calibrated differential pressure measurement.

We did not use a monitoring well as we did in the first application. We took samples of the injected air at the input to GIW815, from the upper (85- to 105-ft depth) and lower (112 to $132 \mathrm{ft}$ ) screened intervals of GIW820, and from the input to the ICE (which represents the combined flow of the upper and lower intervals of GIW820) at 30- to 60-min intervals (Table 5). As Table 4 shows, because of the lower injection pressure in GIW815, it was more difficult to closely regulate the flow rate and the rate varied more widely than it did for the previous sparging. The $22 \mathrm{Ne} / 20 \mathrm{Ne}$ ratio for background air, as measured just before the start of sparging, was 0.104. As shown in Figure 15, values of the percent injected air calculated from the $22 \mathrm{Ne} / 20 \mathrm{Ne}$ ratio of the trace gas measured at the ICE-in and at the GIW820 upper interval track very closely. This confirms that almost all of the flow in GIW820 was coming from the upper interval. The data also indicate that the first trace of injected air arrived at the extraction well some time between 9:22 a.m. and 9:51 a.m., giving a travel time for the injected air of 33 to $62 \mathrm{~min}$. This is significantly shorter than the travel time for the previous case, probably because the air in this case was moving primarily in the vadose zone. The percentage of recovered air in GIW820 upper reached a maximum of about $44 \%$ after about $6 \mathrm{~h}$ of air injection and then stayed more or less constant. The percent injected air had dropped off to about 5\% at the ICE-in by the morning of November 24, about $19 \mathrm{~h}$ after air injection had ceased.

The percentage of trace gas in the lower interval of GIW820 is much more difficult to interpret. The first sample taken at the GIW820 lower, at 9:22 a.m., showed that about $26 \%$ of the vapor present was from the injected air. This is surprising because a sample taken at the same time from the GIW820 upper showed no tracer air present. However, if part of the lower interval of GIW820 was unsaturated, then it is conceivable that some of the air could have reached the lower interval of GIW820 before the upper interval by traveling in the unsaturated portion of this zone. The values of trace gas present in GIW820 lower did not vary by much over the $5.5 \mathrm{~h}$ that measurements were taken.

During the course of this application, we discovered that there was a problem with the way vapor samples were obtained in the field using the Tedlar bags. The problem first came to light when we took the initial vapor samples for hydrocarbon analyses at the beginning of air injection. Measured values for total hydrocarbons (TH) came in lower than 1,000 ppmv; these were significantly lower than what had been obtained several days earlier (values around $5,000 \mathrm{ppmv}$ ). We then had three different technicians each take a sample from the ICE-in, using the same procedure to fill the Tedlar bag, with all samples taken within about $10 \mathrm{~min}$. The TH values for these three samples came out to be 70,225, and 1,120 ppmv. This range of error was clearly due to problems with the sampling procedure. The problem was later identified as being due to ambient air getting into the sample bag because of poor connections with the sampling hose that goes to the vapor flow line. Addition of ambient air to the sample dilutes the results and makes the hydrocarbon content come out too low. The sampling procedures were revised to 
Table 4. Composition of tracer gas during the second air sparging application.

\begin{tabular}{|c|c|c|c|c|c|}
\hline & Date & Time & $22 \mathrm{Ne} / 20 \mathrm{Ne}$ & $\begin{array}{l}\text { Flow rate } \\
\text { (scfm) }\end{array}$ & \\
\hline \multirow[t]{8}{*}{ Injection well } & $11 / 22 / 93$ & 9:12 & 0.388 & 51 & \\
\hline & $11 / 22 / 93$ & $10: 12$ & 0.427 & 44.5 & \\
\hline & $11 / 22 / 93$ & $10: 55$ & 0.405 & 48 & \\
\hline & $11 / 22 / 93$ & $11: 53$ & 0.41 & 47 & \\
\hline & $11 / 22 / 93$ & $14: 58$ & 0.416 & 46 & \\
\hline & $11 / 23 / 93$ & $13: 57$ & 0.462 & 40 & \\
\hline & $11 / 23 / 93$ & $15: 05$ & 0.459 & 41 & \\
\hline & Date & Time & $\begin{array}{c}\text { Elapsed time } \\
\text { (h) }\end{array}$ & $22 \mathrm{Ne} / 20 \mathrm{Ne}$ & $\begin{array}{c}\text { \% Injected } \\
\text { air }\end{array}$ \\
\hline \multirow[t]{12}{*}{ ICE-in } & $11 / 22 / 93$ & $9: 54$ & 1.0 & 0.120 & 5.6 \\
\hline & $11 / 22 / 93$ & $11: 09$ & 2.4 & 0.185 & 26.9 \\
\hline & $11 / 22 / 93$ & $12: 05$ & 3.3 & 0.210 & 34.6 \\
\hline & $11 / 22 / 93$ & $13: 03$ & 4.3 & 0.223 & 38.9 \\
\hline & $11 / 22 / 93$ & $14: 57$ & 6.2 & 0.240 & 43.6 \\
\hline & $11 / 22 / 93$ & $16: 29$ & 7.7 & 0.234 & 42.5 \\
\hline & $11 / 22 / 93$ & $20: 23$ & 11.6 & 0.244 & 45.8 \\
\hline & $11 / 22 / 93$ & $23: 41$ & 14.7 & 0.230 & 41.2 \\
\hline & $11 / 23 / 93$ & $10: 27$ & 25.7 & 0.220 & 37.9 \\
\hline & $11 / 23 / 93$ & 14:02 & 29.2 & 0.254 & 42.1 \\
\hline & $11 / 23 / 93$ & $15: 08$ & 30.3 & 0.254 & 42.1 \\
\hline & $11 / 24 / 93$ & $8: 08$ & 47.3 & 0.118 & 3.9 \\
\hline \multirow[t]{10}{*}{ GTW820 upper } & $11 / 22 / 93$ & $9: 22$ & 0.6 & 0.104 & 0.0 \\
\hline & $11 / 22 / 93$ & 9:51 & 1.0 & 0.118 & 4.3 \\
\hline & $11 / 22 / 93$ & $10: 25$ & 1.6 & 0.154 & 16.3 \\
\hline & $11 / 22 / 93$ & $10: 59$ & 2.2 & 0.175 & 23.2 \\
\hline & $11 / 22 / 93$ & $11: 35$ & 2.8 & 0.201 & 31.7 \\
\hline & $11 / 22 / 93$ & $12: 01$ & 3.2 & 0.210 & 34.6 \\
\hline & $11 / 22 / 93$ & $12: 26$ & 3.6 & 0.219 & 37.6 \\
\hline & $11 / 22 / 93$ & $12: 56$ & 4.1 & 0.225 & 38.3 \\
\hline & $11 / 22 / 93$ & $13: 29$ & 4.7 & 0.231 & 40.2 \\
\hline & $11 / 22 / 93$ & $14: 35$ & 5.8 & 0.241 & 43.4 \\
\hline \multirow[t]{7}{*}{ GIW820 lower } & $11 / 22 / 93$ & $9: 22$ & 0.6 & 0.185 & 28.3 \\
\hline & $11 / 22 / 93$ & $9: 51$ & 1.0 & 0.188 & 25.8 \\
\hline & $11 / 22 / 93$ & $10: 25$ & 1.6 & 0.205 & 33.0 \\
\hline & $11 / 22 / 93$ & $11: 43$ & 2.9 & 0.175 & 23.2 \\
\hline & $11 / 22 / 93$ & $12: 26$ & 3.6 & 0.208 & 34.0 \\
\hline & $11 / 22 / 93$ & $13: 29$ & 4.7 & 0.186 & 26.8 \\
\hline & $11 / 22 / 93$ & $14: 55$ & 6.1 & 0.187 & 26.3 \\
\hline
\end{tabular}


Table 5. TPH composition from canisters (trace gas samples), second sparging application.

\begin{tabular}{|c|c|c|c|c|c|c|c|c|c|}
\hline $\begin{array}{c}\text { Elapsed } \\
\text { time } \\
\text { (h) }\end{array}$ & $\begin{array}{c}\text { ICE } \\
\text { flow rate } \\
\text { (scfm) }\end{array}$ & $\begin{array}{c}\text { GIW820U } \\
\text { TPH } \\
\text { (ppmv) }\end{array}$ & $\begin{array}{c}\text { ICE-in } \\
\text { TPH } \\
\text { (ppmv) }\end{array}$ & $\begin{array}{c}\text { TPH } \\
(\mathrm{mg} / \mathrm{L}) \\
\text { @ STP }\end{array}$ & $\begin{array}{c}\text { Total } \\
\text { mass } \\
\text { removal } \\
\text { (g) }\end{array}$ & $\begin{array}{c}\text { Mass } \\
\text { removal } \\
\text { rate } \\
(\mathrm{g} / \mathrm{h})\end{array}$ & $\begin{array}{l}\text { Cumulative } \\
\text { mass } \\
\text { removal } \\
\text { (g) }\end{array}$ & $\begin{array}{c}\text { Cumulative } \\
\text { mass } \\
\text { removal } \\
\text { (gal) }\end{array}$ & $\begin{array}{c}\text { Sample } \\
\text { from }\end{array}$ \\
\hline 1.0 & 48 & 4,868 & - & 17.15 & 1,398 & 1,398 & $1,398.38$ & 0.47 & GIW820U \\
\hline 1.1 & 48 & - & 4,290 & 15.11 & 123 & 1,232 & $1,521.61$ & 0.51 & ICE-in \\
\hline 2.2 & 49 & 6,066 & - & 21.37 & 1,957 & 1,779 & $3,478.31$ & 1.16 & GIW820U \\
\hline 2.3 & 49 & - & 4,340 & 15.29 & 127 & 1,273 & $3,605.58$ & 1.20 & ICE-in \\
\hline 3.2 & 49 & 4,171 & - & 14.69 & 1,101 & 1,223 & $4,706.39$ & 1.57 & GIW820U \\
\hline 3.3 & 49 & - & 4,430 & 15.60 & 130 & 1,299 & $4,836.30$ & 1.61 & ICE-in \\
\hline 4.1 & 48 & 4,355 & - & 15.34 & 1,001 & 1,251 & $5,837.11$ & 1.95 & GIW820U \\
\hline 4.2 & 48 & - & 4,550 & 16.03 & 131 & 1,307 & $5,967.81$ & 1.99 & ICE-in \\
\hline 4.7 & 48 & 3,618 & - & 12.74 & 520 & 1,039 & $6,487.47$ & 2.16 & GIW820U \\
\hline 6.1 & 47 & - & 3,860 & 13.60 & 1,520 & 1,086 & $8,007.48$ & 2.67 & ICE-in \\
\hline
\end{tabular}

eliminate this problem (see Appendix 4), but by the time this was accomplished we were already about $4 \mathrm{~h}$ into the air sparging application. As a consequence, the most important part of the hydrocarbon data for this application had been compromised.

However, we could still obtain hydrocarbon data from the early part of the sparging by using a portion of the air sampled for the noble gas tracers. Portions of gas from six samples each of the trace gas samples from the upper zone of GIW820 and from the ICE-in were transferred to smaller evacuated cylinders of known volume at a measured pressure. These samples (referred to here as the "canister" samples) were then analyzed for hydrocarbon chemistry in the same manner as the Tedlar bag samples. A comparison of the canister samples with the Tedlar bag can be seen from Table 6 and Figure 16. Figure 16 shows that, until the Tedlar bag sampling procedure was improved about $6 \mathrm{~h}$ after air injection began, the canister samples have consistently higher hydrocarbon concentrations than the Tedlar bag samples. The canister hydrocarbon concentrations show only a slight decrease with time as air sparging proceeds. Canister samples taken at the extraction wellhead (GIW820-upper) have slightly higher concentrations than those taken at similar times at the ICE-in. This suggests that some product is possibly condensing in the plumbing between GIW820 and the ICE-in. In general, there seems to be no correlation between the amount of injected air recovered in GIW820-upper and the hydrocarbon chemistry seen in the canister samples.

For the second sparging case, ERT profiles were run between wells TEP10-TEP6 (also used in the first sparging case), and TEP6-TEP1. The TEP10-TEP6 profile is nearly at right angles to and close to the upstream end of the path from GIW815 to GIW820. The TEP6-TEP1 profile lies parallel to the path between the injection and extraction well and about $20 \mathrm{ft}$ to the west. The ERT difference images are shown in Figure 17. The two upper images are for the profile plane TEP10-TEP6. The one on the left is a difference image between 9:35 a.m. and 1:30 p.m. (4 h) 


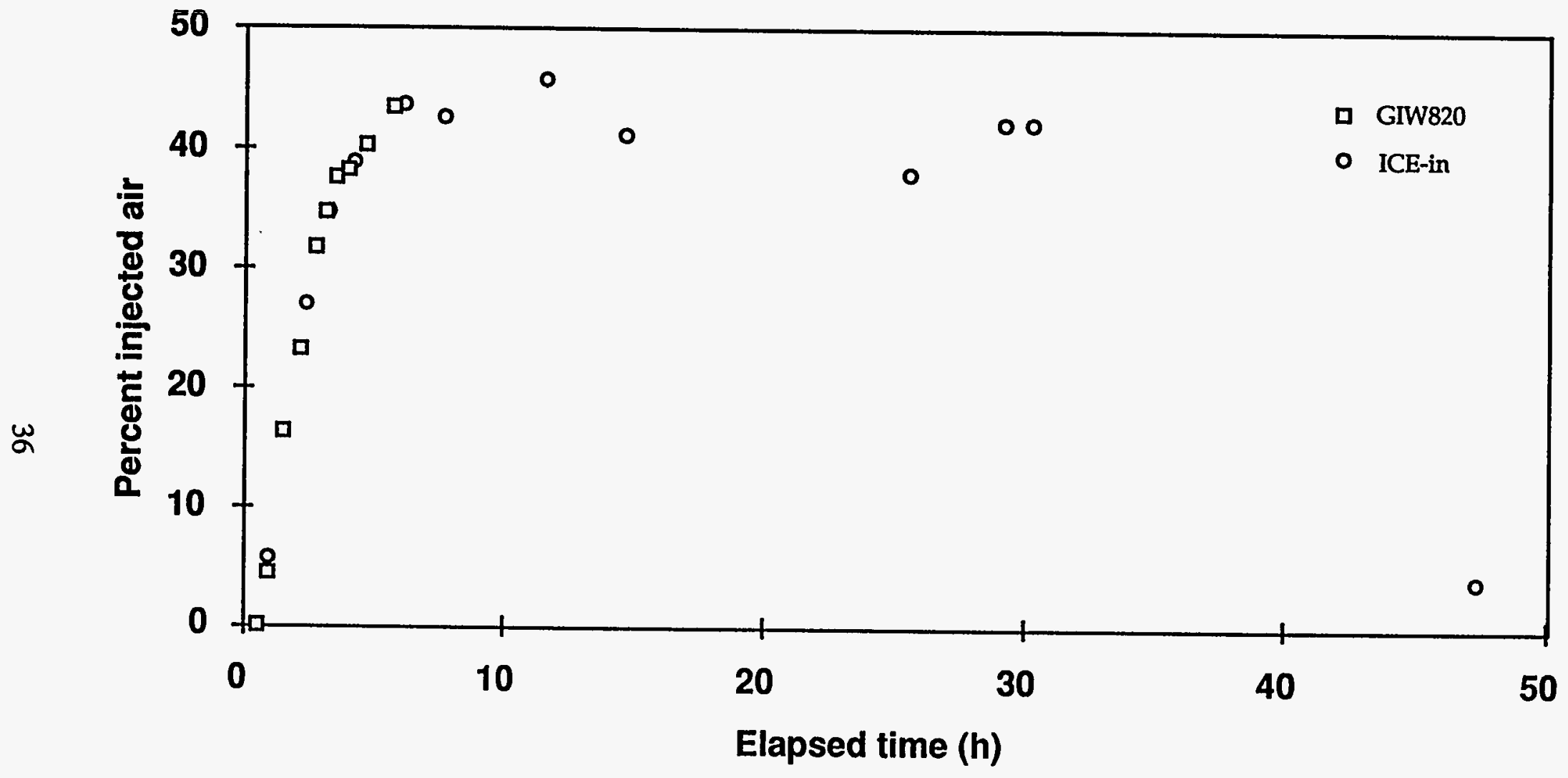

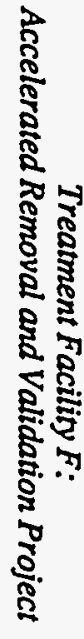

Figure 15. Percent injected air recovered at the upper interval of GIW820 and at the ICE-in versus elapsed time for the second sparging application. Percent injected air is determined from the 22Ne/20Ne ratio in the vapor samples. 
Table 6. Hydrocarbon concentration data during the second sparging application.

\begin{tabular}{|c|c|c|c|c|c|c|c|}
\hline \multirow[b]{2}{*}{$\begin{array}{c}\text { Time of } \\
\text { day }\end{array}$} & \multirow[b]{2}{*}{$\begin{array}{c}\text { Elapsed } \\
\text { time }\end{array}$} & \multicolumn{6}{|c|}{ Hydrocarbon chemistry and total petroleum hydrocarbons } \\
\hline & & $\begin{array}{c}\text { TPH } \\
\text { HC, } \\
(\mathrm{mg} / \mathrm{L})\end{array}$ & $\begin{array}{c}\text { TPH } \\
\text { HC } \\
\text { (ppmV) }\end{array}$ & $\begin{array}{c}\text { Tedlar } \\
\text { bag } \\
\text { ICE from } \\
\text { GIW820 } \\
\end{array}$ & $\begin{array}{l}\text { Canister } \\
\text { sample } \\
\text { ICE from } \\
\text { GIW820 }\end{array}$ & $\begin{array}{c}\text { Tedlar } \\
\text { bag } \\
\text { GIW } \\
\text { upper } \\
\end{array}$ & $\begin{array}{c}\text { Canister } \\
\text { GIW } \\
\text { upper }\end{array}$ \\
\hline $8: 00$ & -0.67 & 18.5 & 5,261 & - & - & - & 5,261 \\
\hline $8: 40$ & -0.17 & 0.4 & 108 & - & - & 108 & - \\
\hline 9:00 & 0.17 & 0.3 & 70 & 70 & - & - & - \\
\hline 9:00 & 0.33 & 0.6 & 175 & 175 & - & - & - \\
\hline 9:00 & 0.33 & 3.1 & 865 & 865 & - & - & - \\
\hline 9:35 & 0.92 & 0.7 & 194 & - & - & 194 & - \\
\hline 9:51 & 1.02 & 17.2 & 4,868 & - & - & - & 4,868 \\
\hline $9: 54$ & 1.07 & 15.1 & 4,290 & - & 4,290 & - & - \\
\hline $10: 05$ & 1.25 & 1.9 & 547 & - & 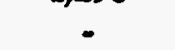 & 547 & - \\
\hline $10: 30$ & 1.67 & 5.4 & 1,520 & - & - & 1,520 & - \\
\hline $10: 59$ & 2.15 & 21.4 & 6,066 & - & - & - & 6,066 \\
\hline 11:00 & 2.17 & 6.9 & 1,950 & - & - & 1,950 & - \\
\hline 11:09 & 2.32 & 15.3 & 4,340 & - & 4,340 & 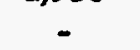 & - \\
\hline 11:15 & 2.42 & 0.3 & 86 & 86 & - & - & - \\
\hline $11: 40$ & 2.83 & 0.1 & 23 & - & - & 23 & - \\
\hline $12: 01$ & 3.18 & 14.7 & 4,171 & - & - & - & 4,171 \\
\hline $12: 05$ & 3.25 & 0.1 & 30 & - & - & 30 & 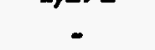 \\
\hline $12: 05$ & 3.25 & 15.6 & 4,430 & - & 4,430 & - & - \\
\hline $12: 35$ & 3.75 & 0.0 & 0 & - & - & 1 & - \\
\hline $12: 56$ & 4.10 & 15.3 & 4,355 & - & - & - & 4,355 \\
\hline $13: 00$ & 4.17 & 0.1 & 29 & - & - & 29 & 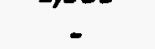 \\
\hline 13:03 & 4.22 & 16.0 & 4,550 & - & 4,550 & - & - \\
\hline $13: 10$ & 4.33 & 0.0 & 16 & - & - & 16 & - \\
\hline $13: 29$ & 4.65 & 12.7 & 3,618 & - & - & - & 3,618 \\
\hline $13: 35$ & 4.75 & 0.3 & 97 & - & - & 97 & - \\
\hline $14: 30$ & 5.67 & 0.1 & 31 & - & - & 31 & - \\
\hline 14:40 & 5.83 & 5.5 & 1,560 & 1,560 & - & - & - \\
\hline $14: 57$ & 6.12 & 13.6 & 3,860 & - & 3,860 & - & - \\
\hline $15: 30$ & 6.83 & 0.1 & 31 & - & - & 31 & - \\
\hline $16: 20$ & 7.50 & 9.8 & 2,770 & 2,770 & - & - & - \\
\hline $16: 29$ & 7.65 & 24.8 & 7,040 & - & 7,040 & - & - \\
\hline $20: 20$ & 11.50 & 2.4 & 693 & 693 & - & - & - \\
\hline $1: 00$ & 14.17 & 7.2 & 2,060 & 2,060 & - & - & - \\
\hline 5:00 & 18.17 & 7.3 & 2,080 & 2,080 & - & - & - \\
\hline $8: 00$ & 21.17 & 6.8 & 1,940 & 1,940 & - & - & - \\
\hline 10:00 & 23.17 & 6.9 & 1,970 & 1,970 & - & - & - \\
\hline $10: 00$ & 23.17 & 6.6 & 1,870 & 1,870 & - & - & - \\
\hline $10: 00$ & 23.17 & 7.0 & 1,990 & 1,990 & - & - & - \\
\hline 21:00 & 34.17 & 9.3 & 2,640 & 2,640 & - & - & - \\
\hline $9: 00$ & 46.17 & 9.3 & 2,640 & 2,640 & - & - & - \\
\hline
\end{tabular}




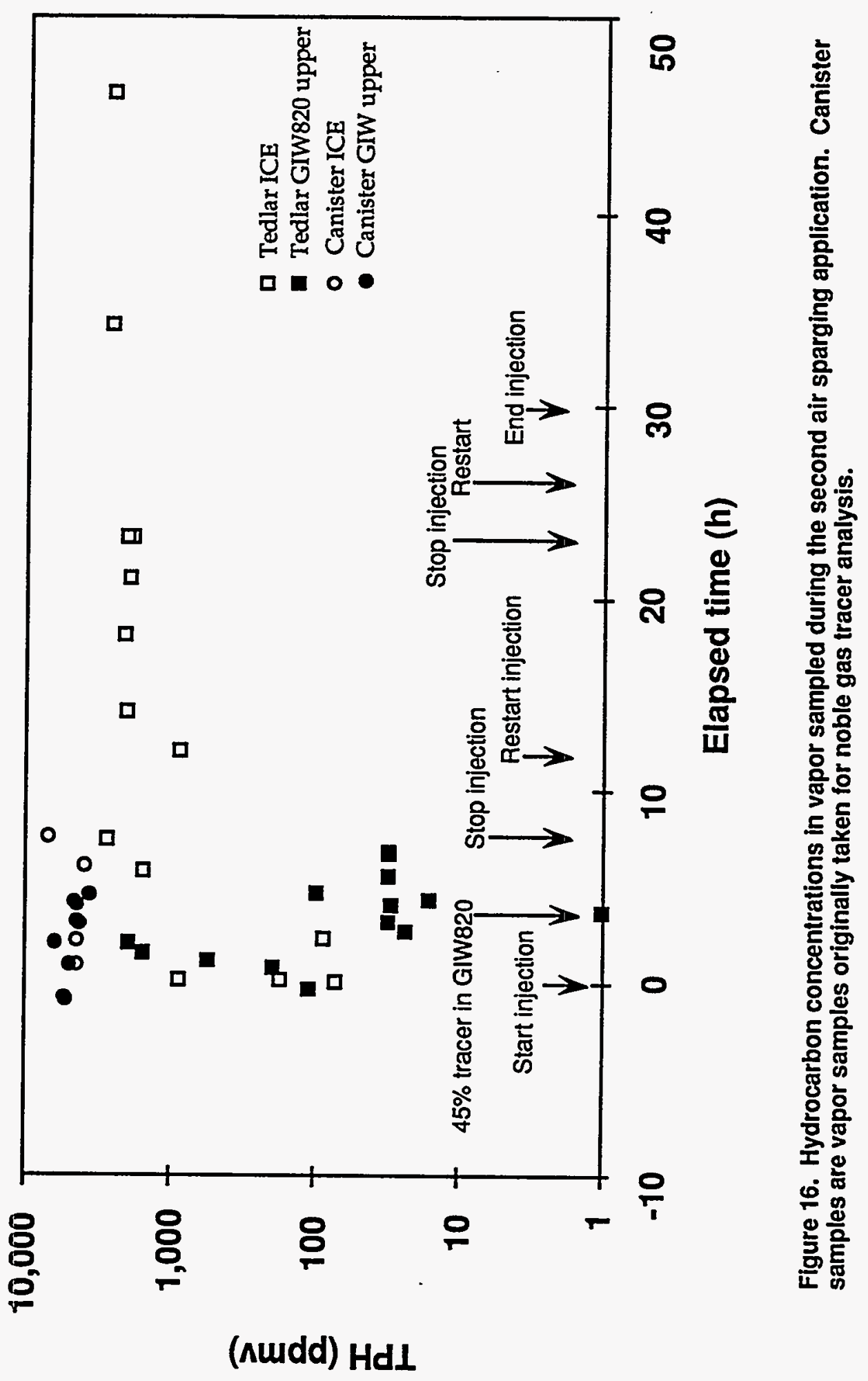





Resistivity change ohm m

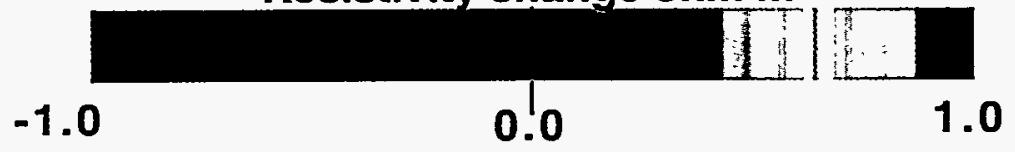

Figure 17. Electrical resistance tomography (ERT) images made from data taken during the second air sparging application. Refer to text for explanation of each part of the figure. 
on November 22. The one on the right represents a longer interval of air injection, with $28 \mathrm{~h}$ difference in time for the image. The image on the left shows little to no effect of the air injection, even though by 1:30 p.m. at least $45 \%$ of the air recovered in GIW820-upper was injected air. The image on the top right of Figure 17 shows a decrease in resistivity of about $0.5 \mathrm{ohm}-\mathrm{m}$ over a very large vertical interval. This probably is caused by the movement of air upward in the vadose zone. The decrease in resistivity, rather than an increase as seen in the previous application, is puzzling. It may be due to the fact that most of the injected air was moving within the vadose zone in this case. Because relatively cold ambient air is being injected near the saturated zone, the air may pick up moisture as it mixes underground, taking more saturated air upward and causing the decrease in resistivity in the vadose zone. The two lower images in Figure 17 are difference images for the TEP6-TEP1 profile for images taken $2 \mathrm{~h}$ apart (left) and $27 \mathrm{~h}$ apart (right). The red zones appearing near TEP1 are caused by local anomalies in the electrodes in TEP1 and are not related to conditions in the matrix. These two lower images do not show any obvious effects related to the air sparging.

In this application, flow rates of the injected air (determined from the trace gas analyses) ranged from 40 to $48 \mathrm{scfm}$ while the flow rates at the extraction well (as measured at the ICE) were 47 to $49 \mathrm{scfm}$. The trace gas analysis indicates that after the first $2.5 \mathrm{~h}$, more than $30 \%$, and up to $45 \%$, of the injected air was being recovered in GIW820. This means that 14 to $20 \mathrm{scfm}$ of the air reaching the ICE after $2.5 \mathrm{~h}$ of injection was injected air. The hydrocarbon concentration in the extracted vapor changed very little during buildup and maximum recovery of injected air; thus, the injected air was not diluting the extracted air, implying that the injected air was picking up hydrocarbons as it moved through the formation. The slight decrease with time in the hydrocarbon concentration in the extract vapor suggests that this contribution from the injected air did slightly decrease over time.

\section{Summary of the Sparging Applications}

Even with the interrupted schedule and problems with hydrocarbon sampling, the two sparging applications are considered a success because of what we learned from them. The ability to track the movement of the injected air is critical, especially in areas like the TFF site where the geology is extremely heterogeneous. In both cases, we found that noble gas tracer analysis is very useful for determining (1) where the injected air moves and how long it takes it to get there, (2) what fraction of the vapor is composed of injected air, and (3) the flow rate of injected air. The noble gas tracer samples also provided us with a valuable backup for hydrocarbon chemistry analysis. These capabilities of the trace gas analysis are only the firstorder portion of the analysis. By analyzing the behavior of isotopes of gases with different mass (such as xenon or krypton), additional information can be gleaned, such as how much of the injected air actually contacted the water, what was its residence time, etc. We did not have time during this project to complete analyses of the other trace gas species or analyses of the water samples mentioned above.

Knowledge of the underground geological structure and the configuration of the water table is also important. These factors affect how the air will move and whether it is affecting the saturated or unsaturated zone. In areas of heterogeneous geology like the TFF site, however, it is not possible to know the subsurface geology well enough to predict in detail how the air will move. For example, fractures or inhomogeneities can provide pathways for air into the vadose 
zone through an apparently impermeable formation, leading to travel pathways that are impossible to decipher. Computer simulations provide a valuable means to test conceptual ideas about subsurface conditions and study the efficacy of different extraction/injection well configurations. In our experience here, we found the computer simulations to be quite accurate in describing the general behavior of the injected air.

The ERT imagery used here can best be described as showing promise. The sparging times were not long enough to develop clear-cut changes in the formation that could be strongly reflected in the ERT imagery. In spite of these limitations, the images did suggest that changes as small as 0.5 ohm-m can be detected under good conditions. Our conclusion is that ERT imagery, in conditions like the TFF site, might be useful for tracking air movement in air sparging. However, for most applications, it will be very difficult to justify the expense of putting in dedicated wells for the sole use of obtaining ERT images for an air sparging operation. 


\section{Electrical Heating}

Electrical preheating was previously employed during the DUSDP at TFF to warm clay layers. Then, two separate steam injection and vacuum extraction phases removed gasoline from the soil and ground water. In the application to this ARV phase, electrical heating was designed to target a particular region, a "cold spot," below the water table, which did not heat up sufficiently in the initial phases of electrical heating and steam injection. This cold spot, consisting of low-permeability soils below the water table, is near heating wells HW104 and HW103 as shown in Figure 18. The cold spot was identified during the drill-back characterizations carried out by the DUSDP in September 1993 (Newmark et al., 1994).

Five different types of wells are shown in Figure 18. The GIW wells combine steam injectors with electrodes for soil heating and surround the bulk of the spill. The HW wells are for electrical heating only and have separate electrodes in the upper and lower clay units. The TEP wells are thermocouple monitoring wells. The GEW and GSW wells are extraction wells, which are located near the center of the pattern. Finally, the most recently drilled wells, HW-102, 103, 104, and 105, are DUSDP post-steam injection characterization wells that were completed as electrical heating wells for use in the ARV phase. The newer wells differ from earlier electrical heating wells in that the electrodes are longer $(18 \mathrm{~m}$ instead of $3.05 \mathrm{~m})$ and larger in diameter $(11.4 \mathrm{~cm}$ instead of $6.3 \mathrm{~cm})$ than the original $\mathrm{HW}$ or GIW electrodes. Details of the well designs and completions can be found in Newmark et al. (1994).

The new wells were placed to better target the cold spot, and the expected primary current paths and phasing are shown in Figure 19. Note that wells GIW-813, 818, and 819 were not used. Their electrodes were badly damaged during the electrical preheat phase of DUSDP.

Pre-DUSDP electrical soil heating started on November 30, 1993, and ended on December 13,1993 . Operations were conducted $24 \mathrm{~h}$ a day during the weekends of December 4 and 11 and only during the night on weekdays. The desired baseline configuration, shown in Figure 19 and consisting of 12 electrodes, was realized for only a fraction of the total running time. Values of interwell electrical resistance turned out to be too low for us to run the baseline configuration for voltages in excess of 208 volts AC (VAC). At voltages above this, we would exceed the 600-A current limit to the electrodes, which would trip the circuit breakers. When the new wells were installed, we did not upgrade the breakers or wiring to the electrodes and were thus limited to 600 A per electrode.

The operational strategy employed for the ARV phase of electrical heating was based on knowledge gained from the electrical heating phase of DUSDP. That is, the region around an electrode will dry out as it warms up and the warming vaporizes water. When this happens, the interwell resistances will increase and seek to limit the current. Thus, we proceeded by using some subset of the baseline configuration until resistances increased (and currents dropped). We then added an additional well and heated it until currents dropped sufficiently to add yet another well. This process was repeated until we could proceed no further because of excess current levels. Using this strategy, we were able to use all 12 electrodes at $208 \mathrm{VAC}$ and 11 out of 12 electrodes at $350 \mathrm{VAC}$. We were not able to operate with the voltage above $350 \mathrm{VAC}$. 


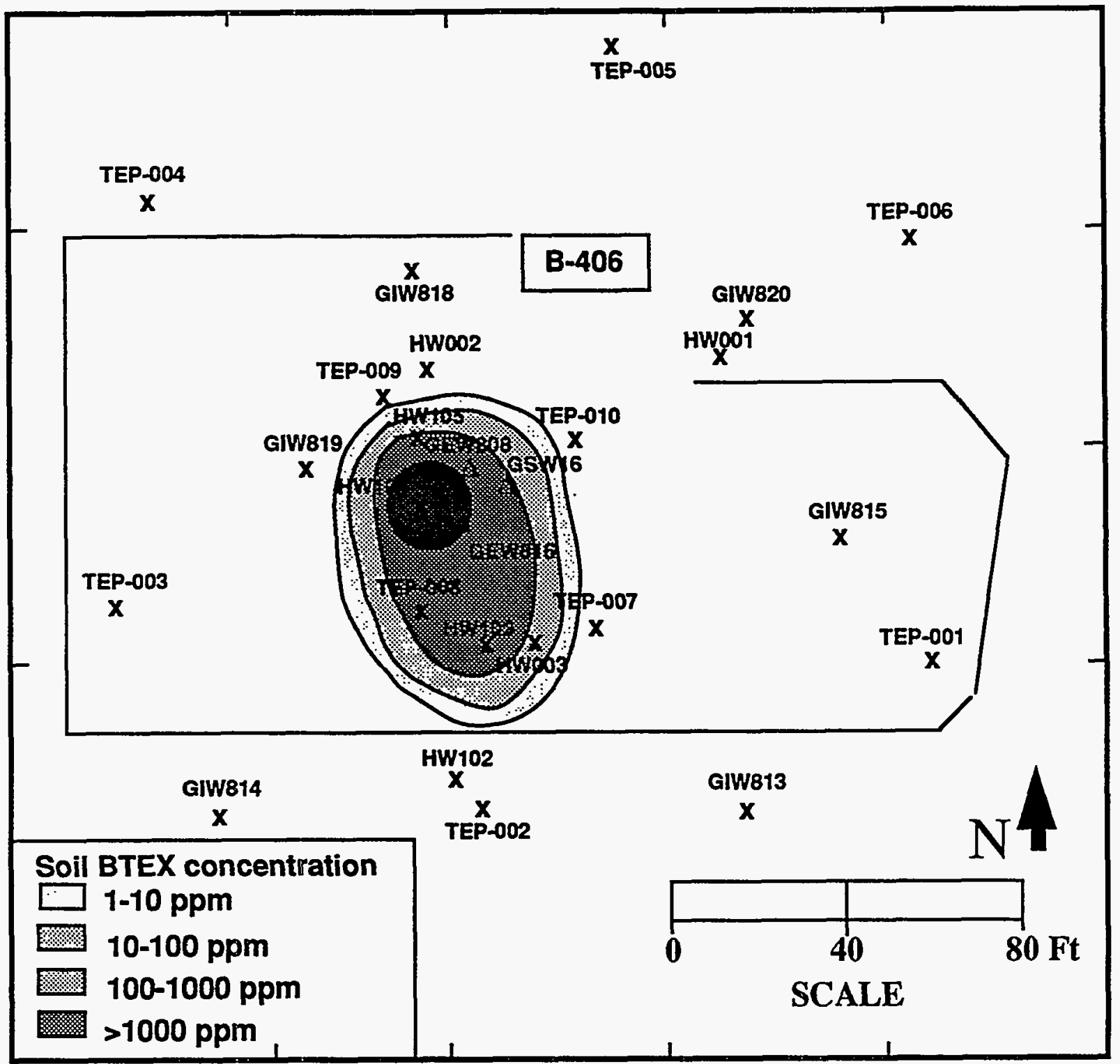

Figure 18. Plan view of the TFF site showing locations of wells of interest for the electrical heating activities. The "cold spot" in the clay aquitard is identified by the area with the highest remaining contamination levels after the DUSDP (figure made using data from Newmark et al., 1994). 


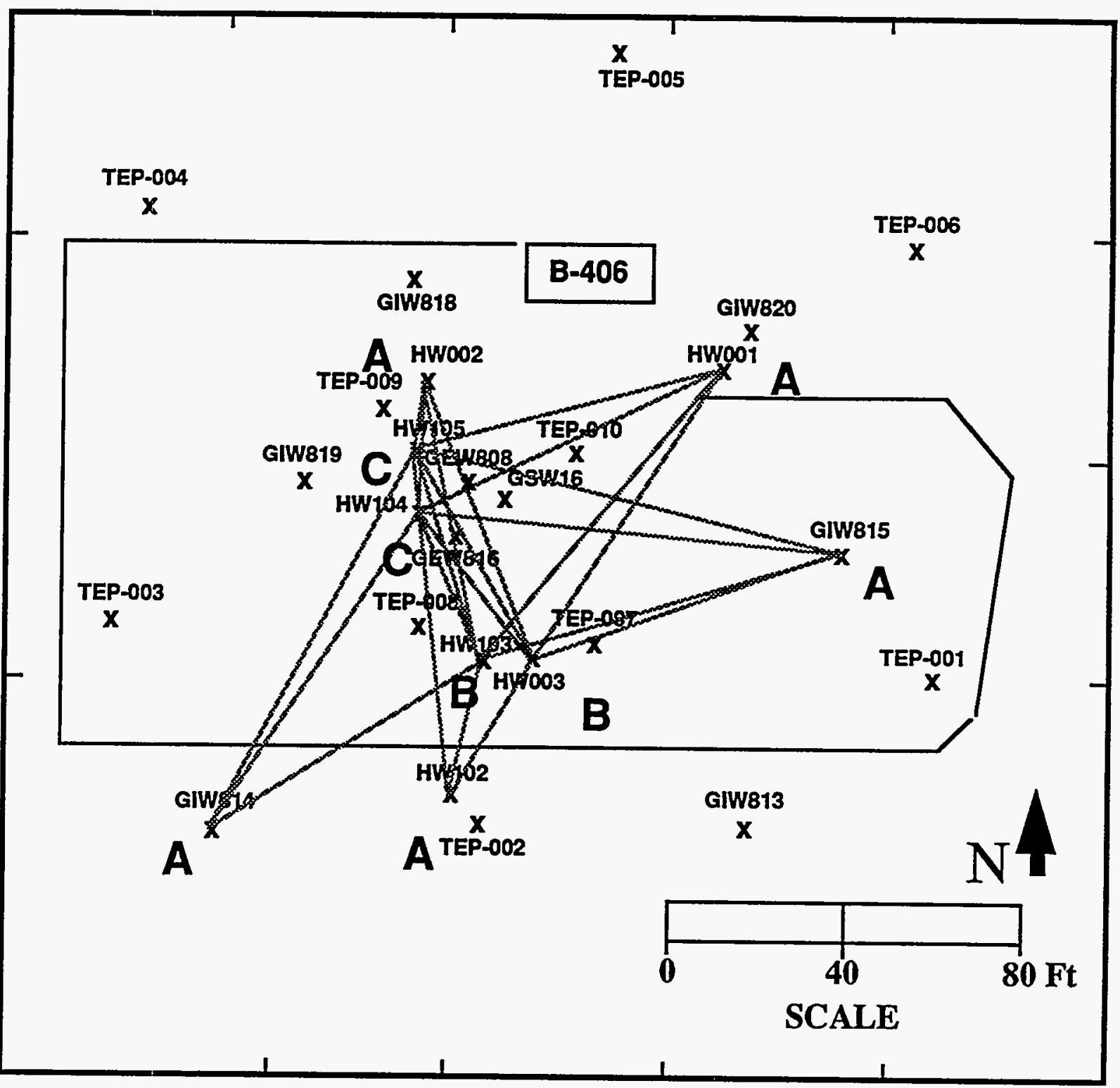

Figure 19. Same plan view as Figure 18, with the inclusion of path lines showing electrical paths available between different heating wells used in the electrical heating operations. 
During the course of the heating, about $44,300 \mathrm{~kW}-\mathrm{h}$ of energy were delivered through the electrodes. In the most productive well, HW-103, 9,145 kW-h of energy were deposited, and in the least productive, HW-002 (upper zone), $716 \mathrm{~kW}-\mathrm{h}$ were deposited. Total power dissipation reached a maximum of $450 \mathrm{~kW}$ with an average value of $221 \mathrm{~kW}$.

Temperatures were logged with the automated data acquisition system (Appendix 3 and Newmark et al., 1994). The results are presented in the plots of Figures 20 to 24 for TEP wells $2,7,8,9$, and 10. Each of these TEP wells has four fixed thermocouples, TCs 1 to 4 , located at depths of $130 \mathrm{ft}, 110 \mathrm{ft}, 95 \mathrm{ft}$, and $80 \mathrm{ft}$, respectively.

Let us now examine the thermocouple data for evidence of warming. Note that thermocouple 1 at the $130-\mathrm{ft}$ depth is below the water table and significantly cooler than the other thermocouples for all of the TEP wells. For the most part, thermocouples 2, 3, and 4 are in the $80^{\circ}$ to $90^{\circ} \mathrm{C}$ range, even many months after steaming.

First, consider TEP 2, which is shown in Figure 20. Thermocouple 1 appears to show the effect of ground water pumping very clearly. During pumping, as cool water is drawn in from the surrounding areas, the temperature decreases (see also Section I above and Fig. 9). When pumping stops, the temperature increases, probably due to conductions from warm soil nearby. Thermocouple 2 is always cooling, though the rate of cooling slows during heating. Thermocouples 3 and 4 clearly show an increase in temperature of around $4^{\circ} \mathrm{C}$ due to electrical heating. TEP 2 is only about $3 \mathrm{~m}$ away from HW-102.

The temperature plots for TEP 7 are shown in Figure 21. Thermocouple 1 appears to respond to ground water pumping, though not as strongly as TEP 2 . Thermocouples 2,3 , and 4 appear to be unaffected by electrical heating or ground water pumping. The heating well nearest to TEP 7 is HW-003, which is about $5.2 \mathrm{~m}$ away.

Figure 22 shows the temperature plots for TEP8. As before, thermocouple 1 responds to ground water pumping. Thermocouple 2 shows a temperature increase of about $3^{\circ} \mathrm{C}$ due to electrical heating. Thermocouples 3 and 4 show a strange temperature increase, which appears to occur just when electrical heating is stopped The origin of this increase is not understood. The heating well nearest to TEP 8 is HW-103 at a distance of about $3.5 \mathrm{~m}$.

Temperature plots for TEP 9 (Fig. 23) are similar to those for TEP7 and TEP8. Thermocouple 1 responds to ground water pumping. Thermocouple 2 cools slowly when electrical heating is off and warms slowly when the heating is on. Thermocouples 3 and 4 cool slowly whether heating is on or off. TEP 9 is about $3.35 \mathrm{~m}$ away from HW-105 and $3.66 \mathrm{~m}$ away from HW-002.

In Figure 24, we see that thermocouple 1 in TEP 10 responds to pumping in a way generally opposite to the other TEP wells. Its temperature increases during pumping and decreases without pumping. Thermocouples 2,3 , and 4 vary only a little, though thermocouple 2 appears to cool slowly after heating is turned off while thermocouples 3 and 4 appear to warm slowly.

A rough estimate of the expected heating effect can be made by assuming that the soil at the TFF site is "average." The volumetric heat capacity for average soil (Carslaw and Jaeger, 1959) is $0.56 \mathrm{~kW}-\mathrm{h} / \mathrm{m}^{3}-^{\circ} \mathrm{C}$. Given the energy input of $44,300 \mathrm{~kW}-\mathrm{h}$, the heating effect can be expressed 


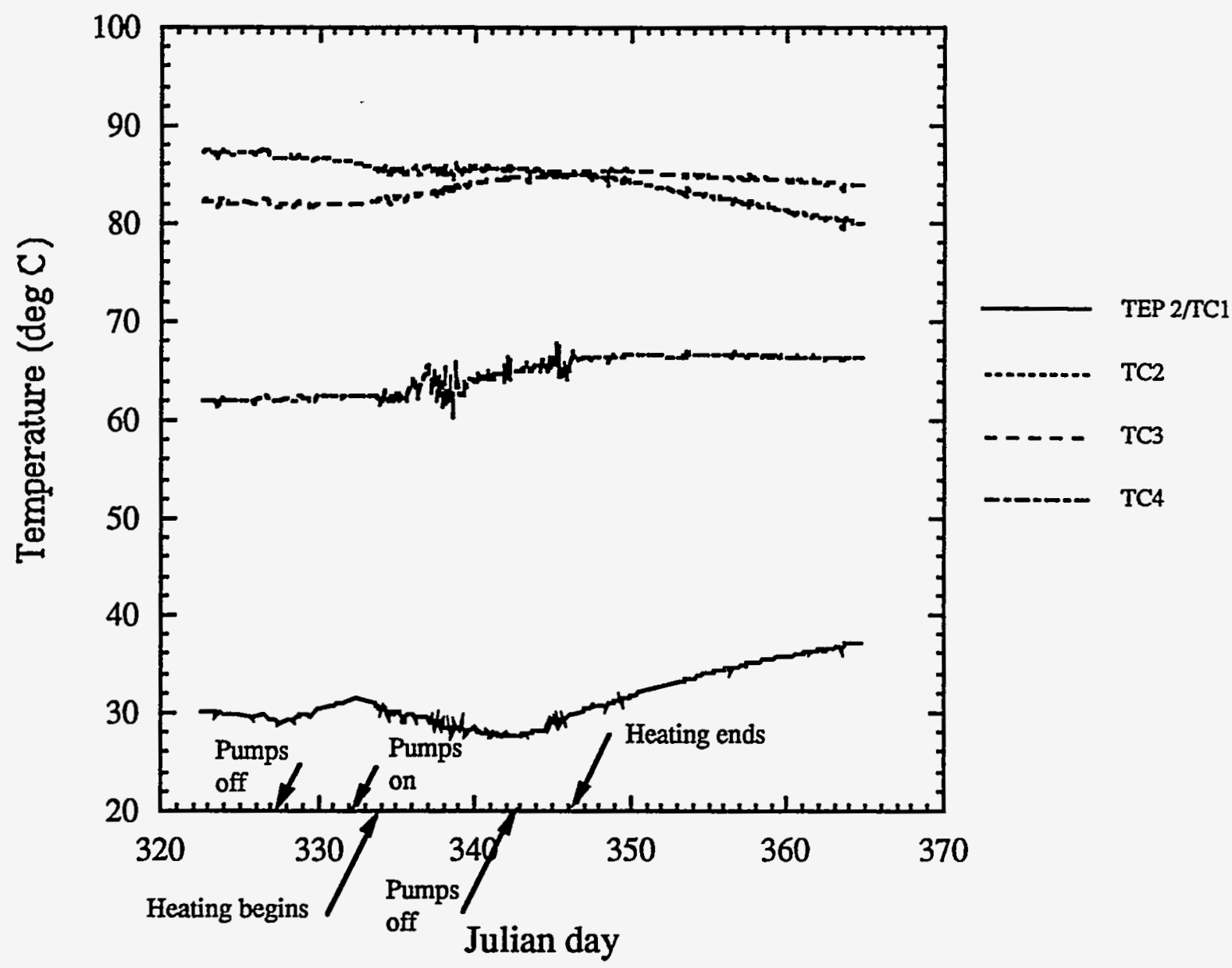

Figure 20. Temperatures at well TEP2 before, during, and after the electrical heating phase of ARV. Arrows show when ground water pumping started and stopped and when electrical heating started and stopped. 


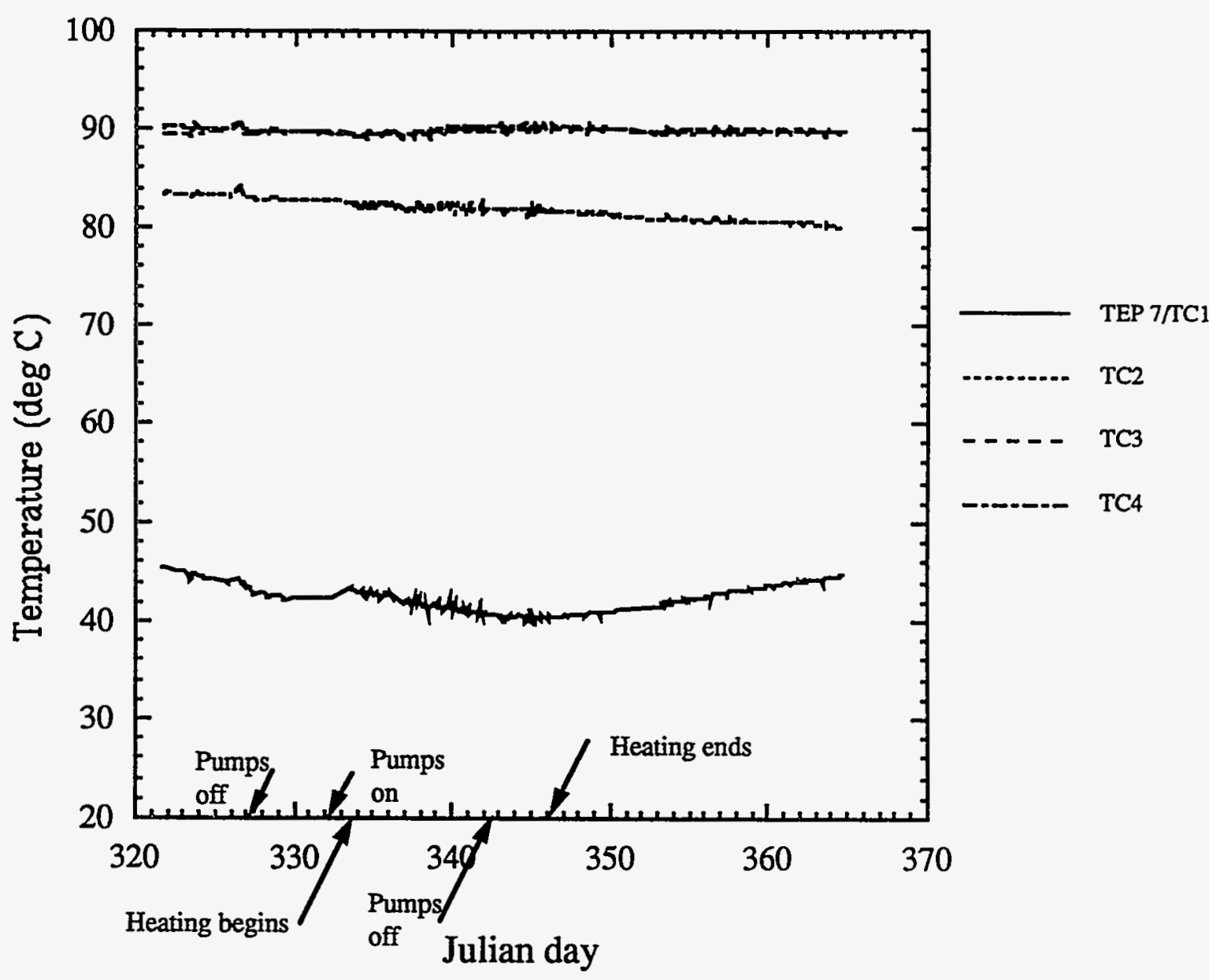

Figure 21. Temperatures at well TEP7 before, during, and after the electrical heating phase of ARV. Arrows show when ground water pumping started and stopped and when electrical heating started and stopped. 


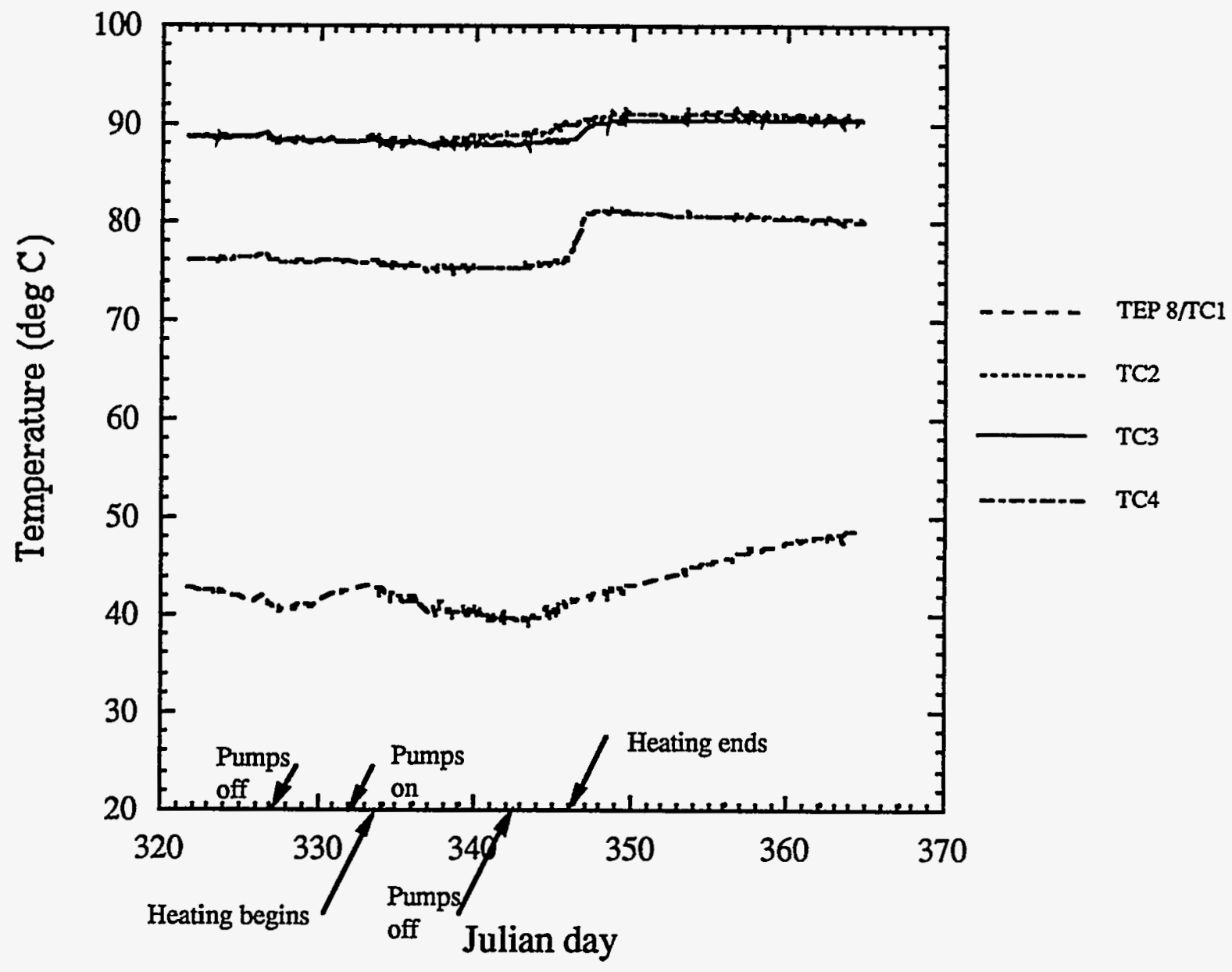

Figure 22. Temperatures at well TEP8 before, during, and after the electrical heating phase of ARV. Arrows show when ground water pumping started and stopped and when electrical heating started and stopped. 


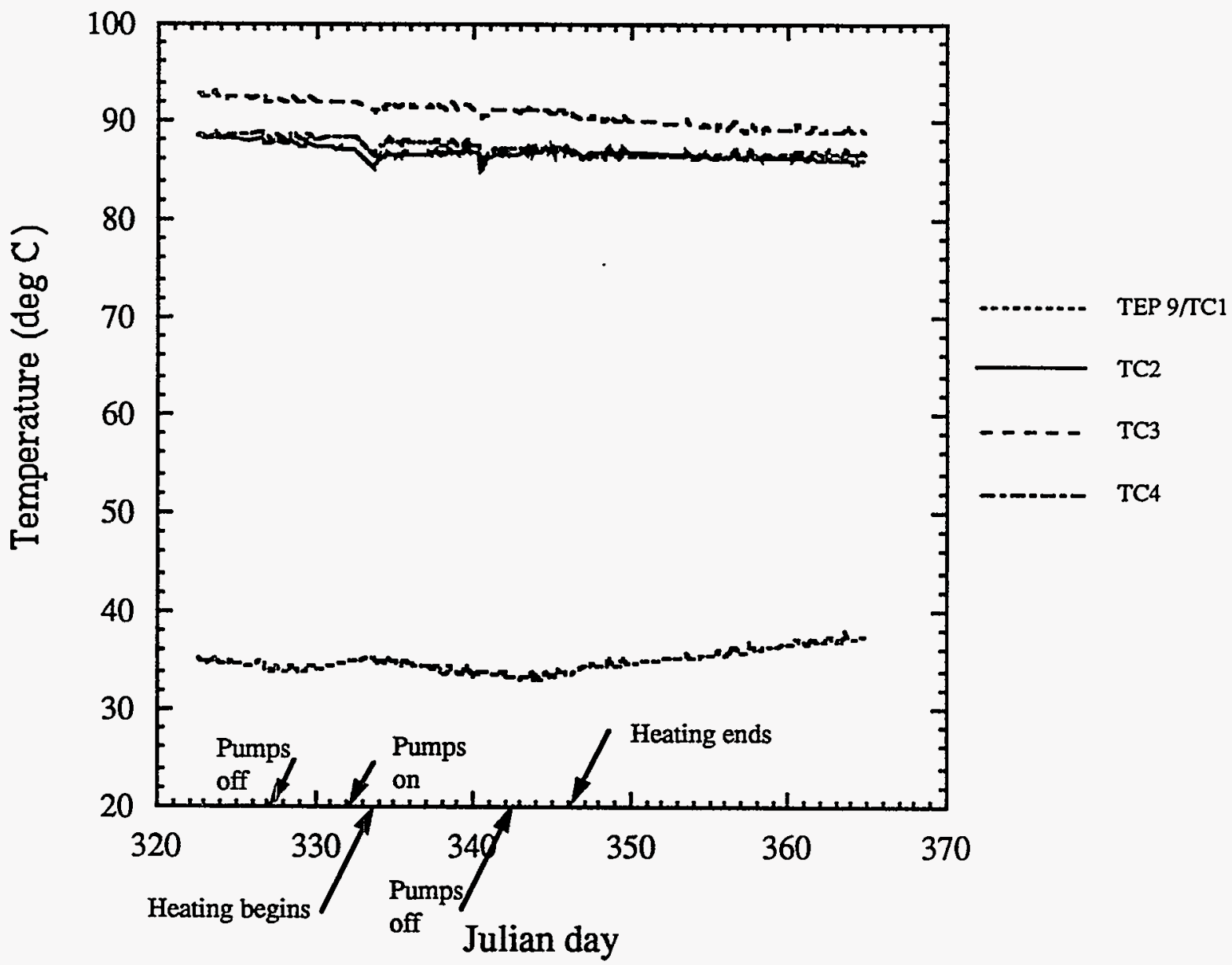

Figure 23. Temperatures at well TEP9 before, during, and after the electrical heating phase of ARV. Arrows show when ground water pumping started and stopped and when electrical heating started and stopped. 


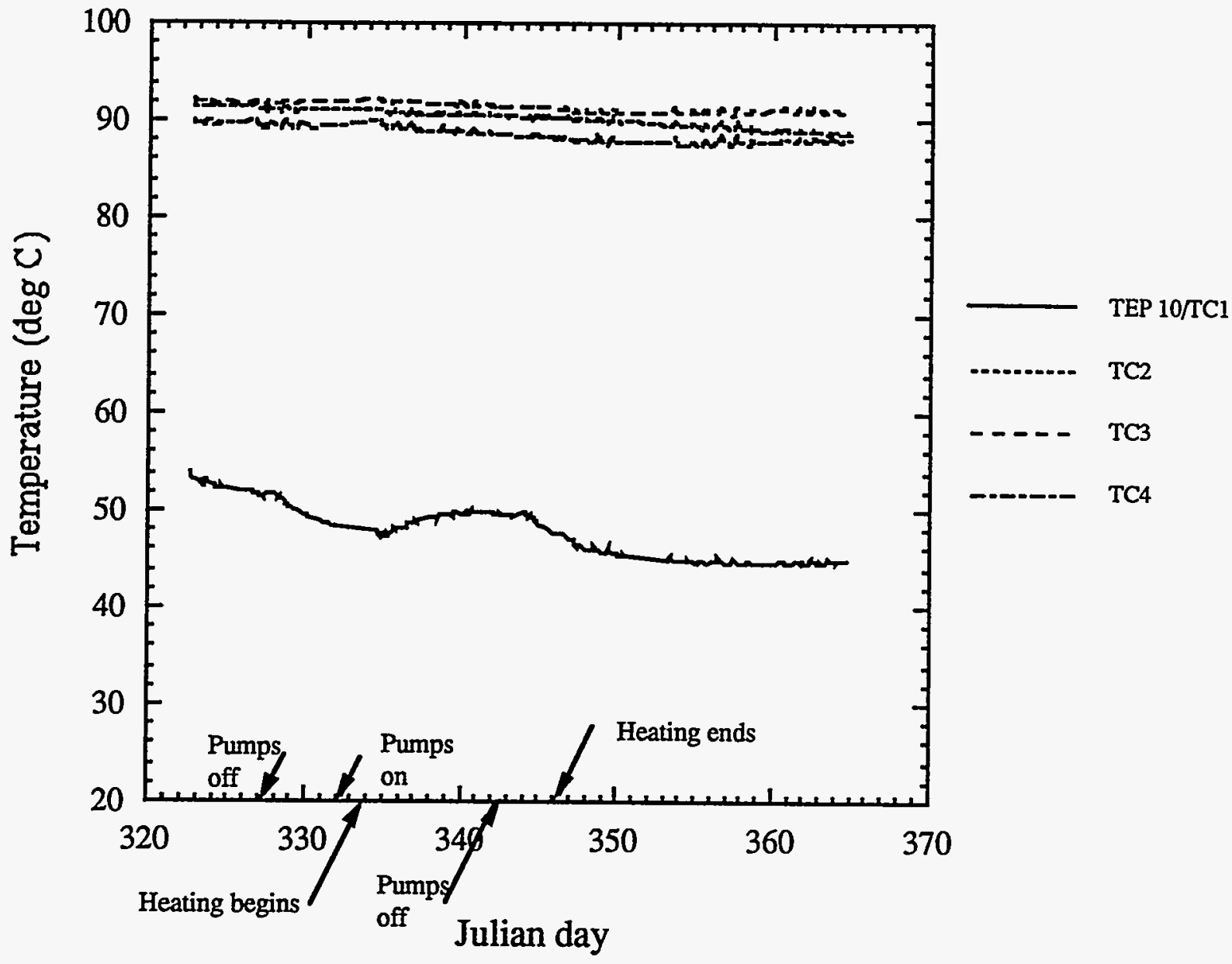

Figure 24. Temperatures at well TEP10 before, during, and after the electrical heating phase of ARV. Arrows show when ground water pumping started and stopped and when electrical heating started and stopped. 
by a volume-temperature product of $79,100 \mathrm{~m}^{3}-^{\circ} \mathrm{C}$. If the volume heated is taken to be $18 \mathrm{~m}$ (60 ft) in height (the electrode length) and bounded by TEP wells $2,7,8,9$, and 10, then the volume is $4,706 \mathrm{~m}^{3}$ and the expected temperature rise is about $79,100 / 4,706^{\circ} \mathrm{C}$, or $16.8^{\circ} \mathrm{C}$. From the temperature plots, temperature increases attributable to heating are obviously a few degrees at most. Where then did all of the energy go?

An examination of the data for condensate produced during electrical heating provides a plausible explanation. The data are presented in Figure 25 and show high condensate collection rates when heating is on, and low collection rates when off. When these data are compared with the energy input versus time of Figure 26, a pulse of condensate seems to be produced each time the heating comes on.

The total condensate collected during electrical heating was 33,085 gals. Of this, about $2,700 \mathrm{gal}$ is what would normally be collected without electrical heating (background). Thus, about 30,385 gal of condensate may be attributed to electrical heating. The energy required to vaporize this amount of water is about $80,000 \mathrm{~kW}-\mathrm{h}$. This figure is nearly twice that for the input electrical energy and needs more explanation, but the main point is: most of the input energy probably went to vaporize water and not to heat soil.

There is a plausible mechanism for this large vapor production. Consider the situation depicted in Figure 27. Here, two long electrodes (18 m), like the ones used in HW-102, 103, 104 , and 105, are placed in a layered soil as shown. This represents the kind of situation at the LLNL gasoline spill. When a voltage is applied between the electrodes (in the model), current flows and the region around the electrodes begins to warm. Steam is produced at the electrodes and moves readily into the upper steam zone (USZ). This has the effect of greatly increasing the electrical conductivity of the USZ relative to the confining clay layer (CON) beneath. As a result, current flows preferentially into the USZ, which heats rapidly to temperatures of $100^{\circ} \mathrm{C}$ while the clay has warmed only about $5^{\circ} \mathrm{C}$.

The above ideas were incorporated into a simulation using the NUFT computer code. The results of this modeling, though preliminary, indicate that it is not good practice to have long electrodes that extend into permeable zones if the object is to heat the clay zones. Details of the modeling work are presented in Appendix 5.

The electrical heating phase of ARV did not produce the desired heating. There were several contributing factors. The first, and perhaps the most important, was that the new electrodes in wells HW-102, 103, 104, and 105 were too long and not properly placed to target the desired clay zone. Because the electrodes extended into the upper and lower steam zones, large quantities of steam formed at the electrodes, and most of the input energy went into making steam. Additionally, when the steam moved into the steam zones, it made them highly conductive and diverted current from the clay zones to the steam zones. The overall result was that the target clay zones heated very slowly, if at all.

Another factor contributing to the poor heating rates was the mismatch between system impedance and the wiring and breakers to the new heating wells, HW-102, 103, 104, and 105. The wiring and safety circuit breakers were limited to $600 \mathrm{~A}$. The electrodes in these wells were capable of much higher currents, had we been able to use the available secondary voltages of 480 and $600 \mathrm{VAC}$. 


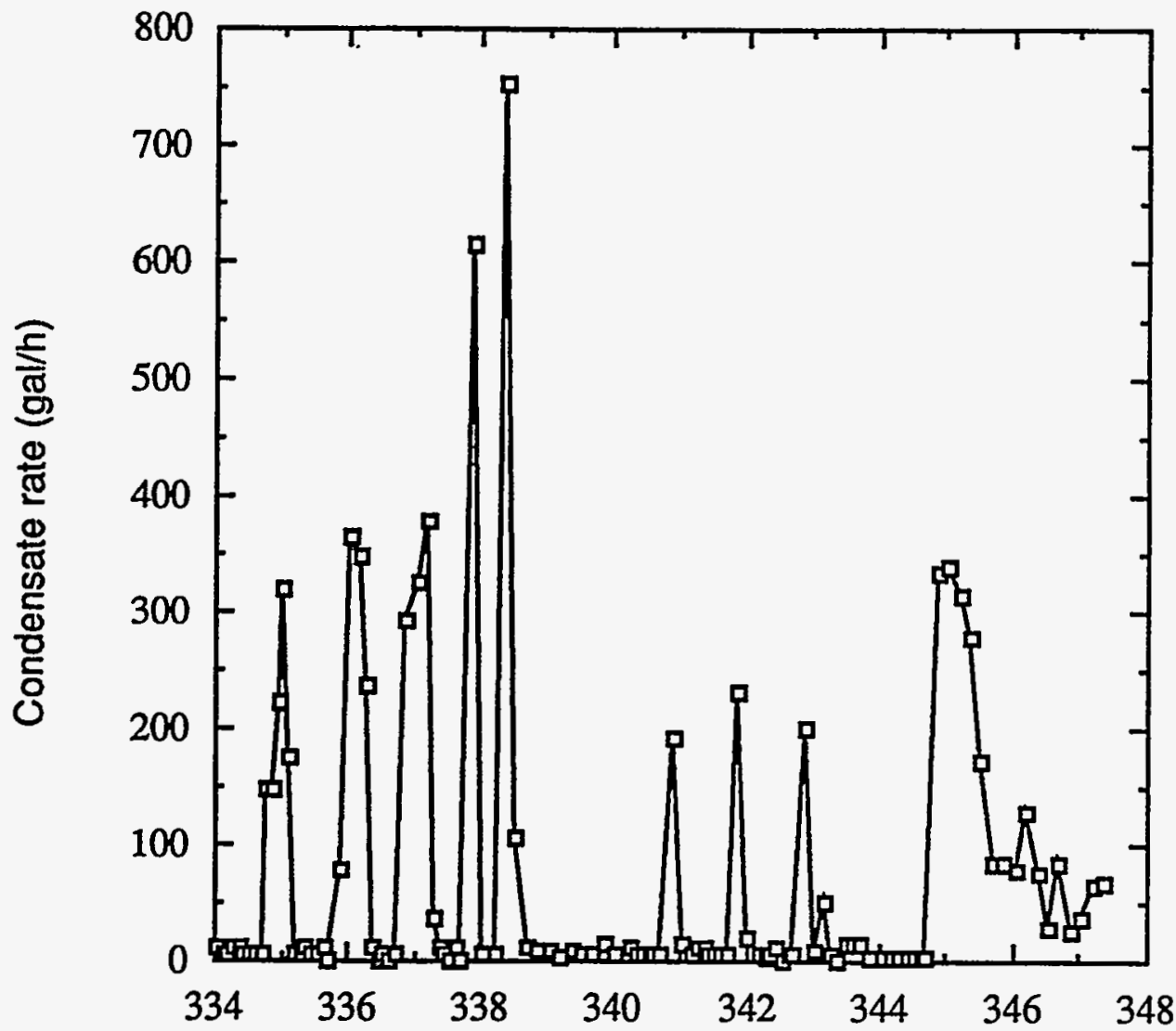

Julian day

Figure 25. Plot of the rate of condensate recovery in the extracted vapor, in $\mathrm{gal} / \mathrm{h}$, as a function of time (Julian day) during the electrical heating phase of ARV. Most of the recovery appears as spikes that occur during overnight or week-end electrical heating activities. 


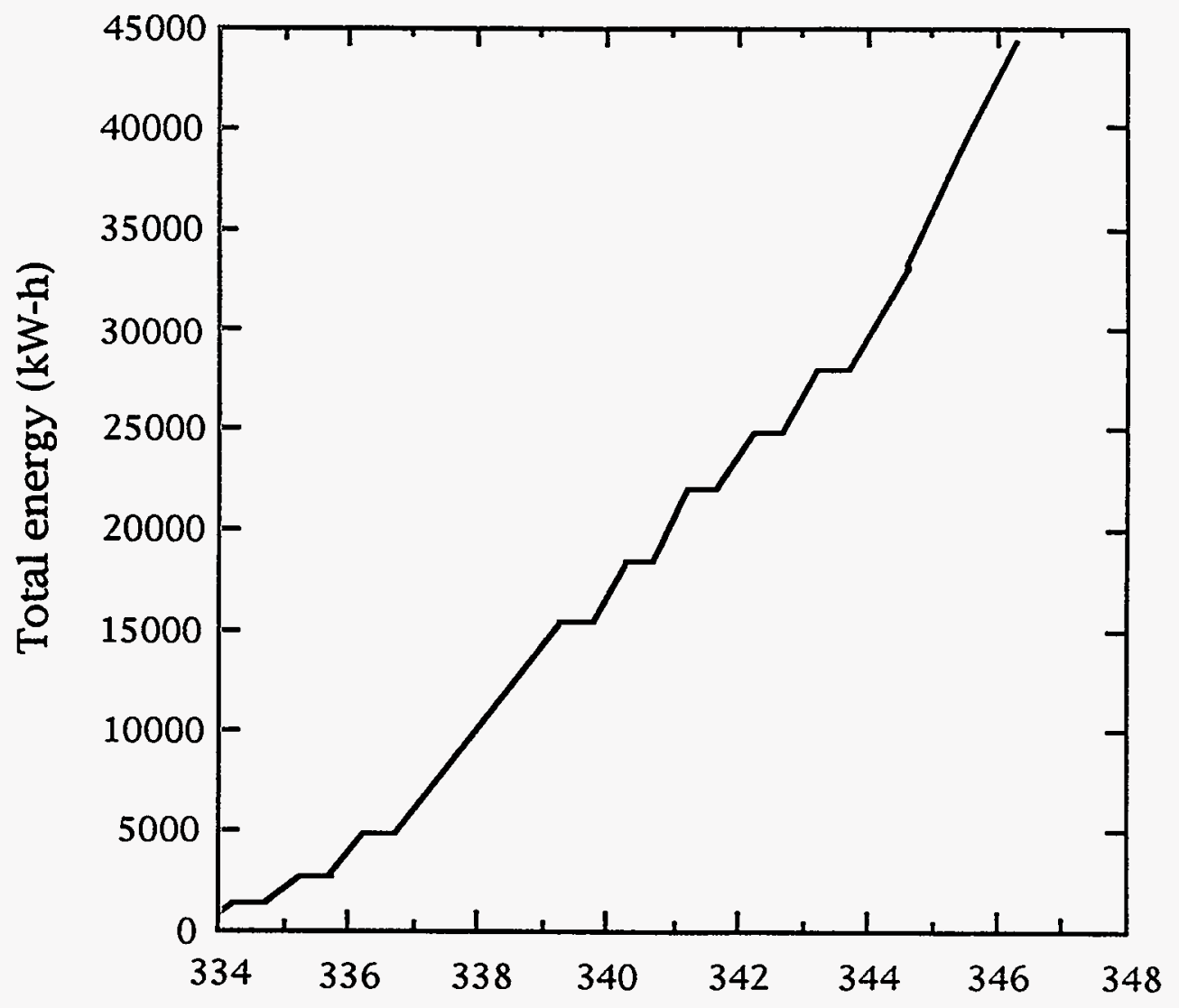

Julian day

Figure 26. Cumulative energy deposition into electrodes, in $\mathbf{k W}-\mathbf{h}$ during the electrical heating part of ARV. Compare with Figure 25, and note that most of the increases in energy (i.e., electrical energy was being applied) correlate with peaks in condensate recovery. 


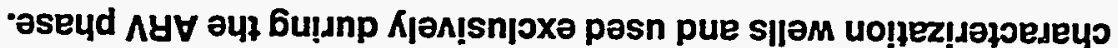

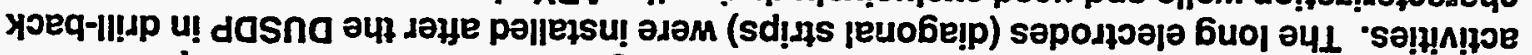

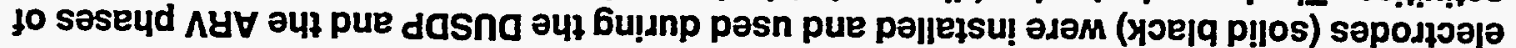

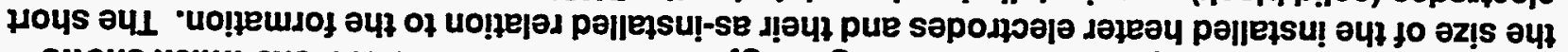

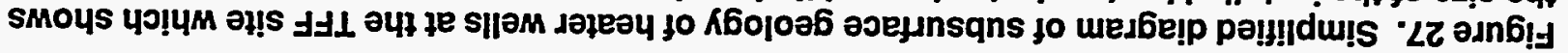

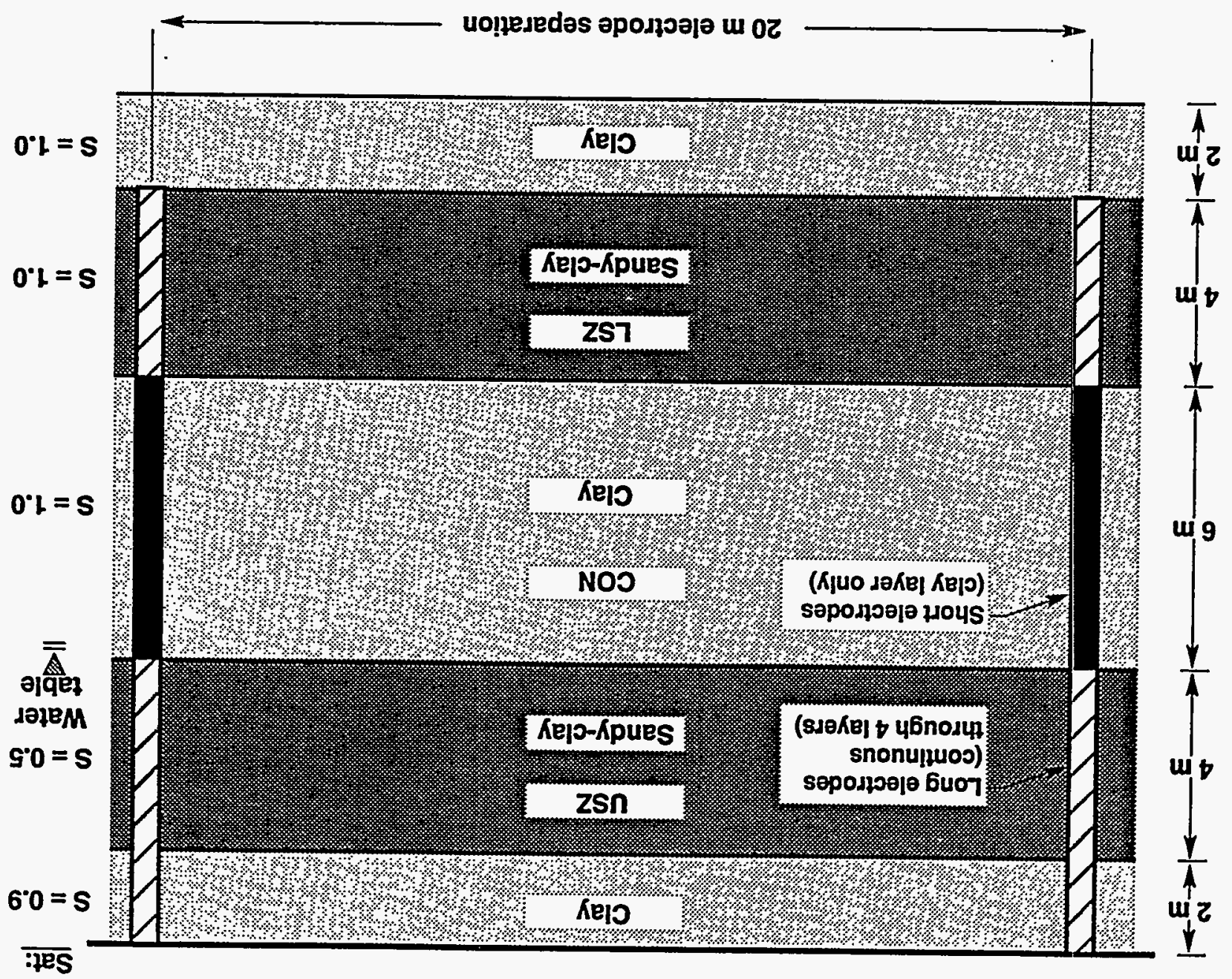


ERT images were also run before and after the electrical heating. Figure 28 shows difference images between data taken on October 27 to 29 and December 13. Note that continuous pumping (with the exception of the Thanksgiving hiatus and the last 4 days) of ground water was carried out during this time and accounts for the largest effect seen on the ERT images. The TEP2-TEP8 and TEP2-TEP7 cross sections show a large area of higher resistivity correlated with zones of high permeability. This is undoubtedly caused by the influx of cold water during pumping (see also Fig. 9). Cooler temperatures will cause resistivity to increase. Other influences, such as the vertical column of increased resistivity seen in TEP2-TEP8 and TEP9-TEP7, may be related to drying of the formation near the electrodes during electrical heating. There is no evidence in the images that specific clay layers have been significantly heated. 


\section{Resistivity changes December 13, 1993}

October 27-29, 1993, data were used as the baseline for differences.
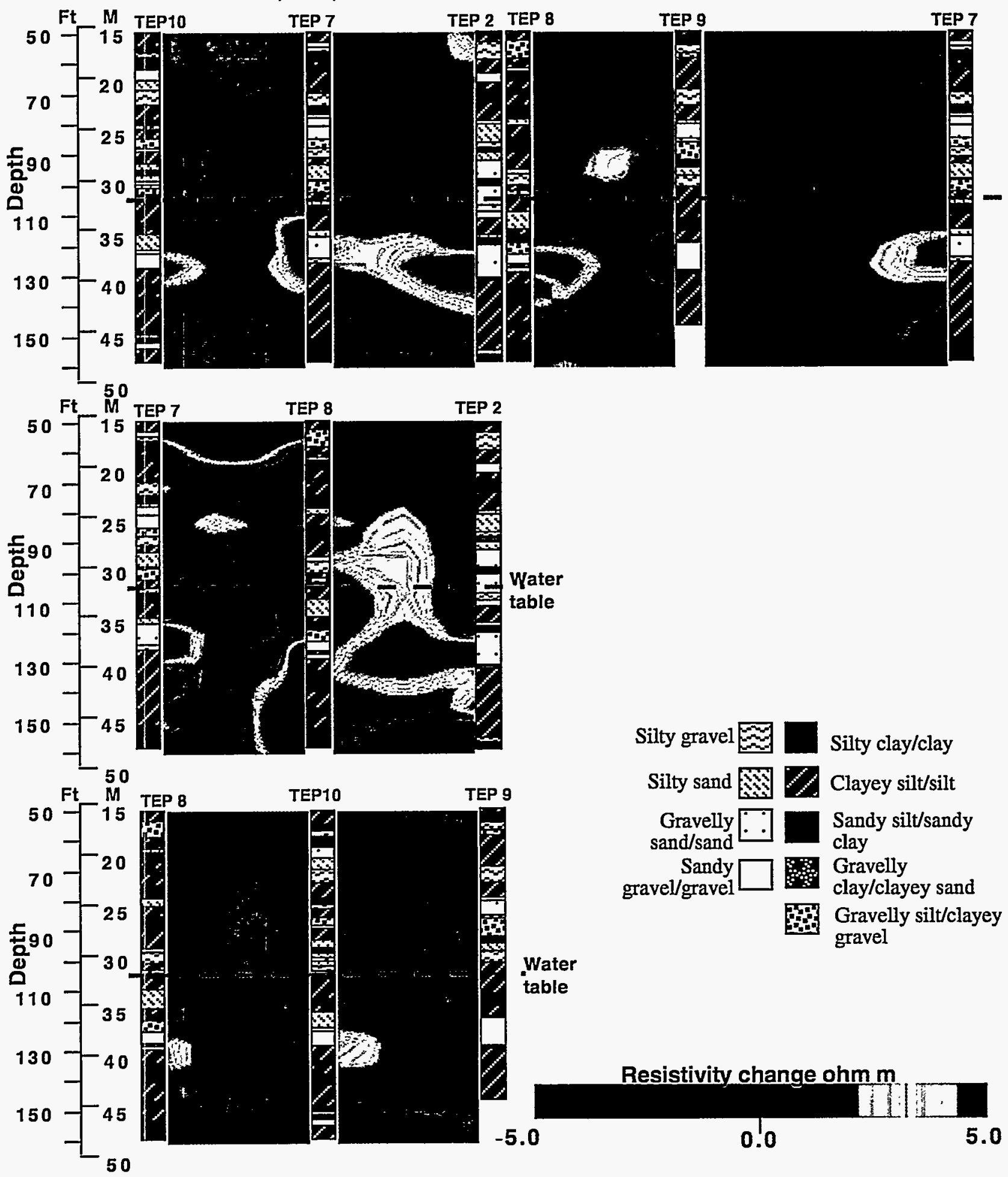

Figure 28. Electrical resistance tomography (ERT) images made at the end of electrical heating. 


\section{Conclusions and Recommendations for Further Work}

In reference to the name of this project-Accelerated Removal and Validation-what we accomplished is best characterized as emphasizing remediation over validation. Our primary goal was to take advantage of the infrastructure and site conditions existant at TFF after the DUSDP to remove as much gasoline as we could with $24-\mathrm{h}$ operations over a few months. In that context, we were quite successful. The estimated 1,000 gal of gasoline removed, which exceeds the amount estimated to be remaining in the areas not effectively cleaned by the DUSDP (about 750 gal, Newmark et al., 1994), is about the same as that removed during the previous 3 months in which contaminant levels were steadily decreasing.

The strategy we used for advanced remediation during the ARV phase was driven by the time constraints that came with the funding for this work. This strategy, with limited time for lowering the water table, carrying out vapor extraction with different well configurations, air sparging, and renewed electrical heating, made it very difficult to assess the relative impact of each set of activities on the relative rate of contaminant removal. For example, we drew down water levels in the center of the TFF site and exposed the clay aquitard more effectively to vapor extraction, but because we were simultaneously carrying out vapor extraction, it is impossible to know how much effect this had on the ultimate recovery of hydrocarbons. It was not possible to have a control case in which we extracted vapor without drawing down the water table to use for comparison.

When operations were resumed in mid-January 1994, contaminant levels in the vapor and water phases were lower than any measured at the extraction wells at TFF. However, these levels may be misleading. The water table had been allowed to return to its pre-ARV phase levels. Thus, the clay aquitard and some of the upper part of the lower steam zone were no longer exposed to vapor extraction. For this reason alone, we would expect that concentrations in the vapor being recovered now would be lower. In addition, we removed and treated about one pore-volume of water from a very large area under the TFF site. This volume of water was replaced with relatively clean water, coming mainly from the south (Fig. 9). Some of this water may contain hydrocarbons driven outward from the site during the DUSDP, but these amounts are estimated to be relatively low (see Newmark et al., 1994). It will take some time for aqueous concentrations to rise again, with sources remaining in small spatially localized areas.

The design of the electrodes in the newer heating wells apparently played a major role in the failure of electrical heating to significantly heat the formation, and specifically, the target "cold spot" in the clay zone. Clearly, electrode design (i.e., length and location of the conductor with respect to conductive parts of the formation and well completion details) and the configuration of the heterogeneous geology play a very important role in the electrical heating process. At this point, we are not ready to make any claims about the ability of electrical heating to enhance recovery of VOCs at the TFF site.

In the following paragraphs, we propose several activities that can be carried out during further activities at the TFF site that will help us (1) evaluate the location and significance of any remaining contamination in the cold spot, (2) assess the effect of lowering the water table on 
removal by vapor extraction, (3) further validate our modeling of electrical heating, (4) develop a strategy to successfully heat the formation with electrical heating and assess its impact on the site, and (5) assess the ability of in situ bioremediation, perhaps enhanced with air sparging, to "polish" up the remaining clean-up activities.

The assessment of the location of the cold spot was done using preliminary assessments of DUSDP activities and chemical sampling results from the characterization wells that were drilled at the end of the DUSDP (which corresponded to the start of ARV). Final assessments of the DUSDP are now being completed, and a new assessment of the location, volume, and extent of contamination (e.g., a mass inventory) of the cold spot can now be completed with more confidence. Once this has been done, we recommend that an additional characterization well or wells be drilled at a site chosen to maximize our post-ARV knowledge about the cold spot, assess the effects of the ARV phase, and be used as a new electrical heating well. The additional wells will provide knowledge of the mass inventory in the zone, which can be used to assess the effect of subsequent electrical heating.

We further propose to again lower the water table by 24-h pumping (which we will be able to do once we automate the pump-and-treat system) to maximum drawdown levels obtained during the ARV without vapor extraction, and then maintain this configuration for about 2 weeks. Then, commencing vapor extraction with the lowered water table and comparing the contaminant removal results with the steady-state prior to drawdown, we will be able to assess the effect of lowering the water level on THC removal rate. If the results show no difference, it will enhance our confidence that the site has indeed been cleaned to a significant degree.

We clearly need to validate the model of electrical heating. A variety of experiments using the existing equipment could be conducted. Several more model runs could confirm the highest payoff approach.

Additional studies of processes occurring around the heater electrodes are necessary to improve our understanding of the physics of the heating process and to validate our computer models. By applying current to sets of two electrodes at a time, such as two of the new long electrodes, two of the original short electrodes, and a combination of short and long electrodes, we can more closely match conditions of the computer simulation. During electrical heating, we will carefully monitor conditions in the borehole (temperature, current, vapor quantity, and composition) to try to discern what is happening in the near field. Once these field tests have been carried out and checked against the model simulations, the model can be adjusted accordingly to give us more confidence in its ability to predict what will happen for a given electrode configuration.

After completing the above work, we will be in a better position to design an electrode configuration to be put into the post-ARV characterization well or wells. Using the computer simulation, we will design the electrodes to be more compatible with the existing configuration (probably using only the original short electrodes), which will optimize our ability to heat the clay zone in the cold spot. We will also be able to simulate electrical heating in conjunction with vapor extraction and water table drawdown for better contaminant removal.

We have submitted a proposal for EM50 funding of a project to improve our knowledge of electrical heating and to develop improved computational tools for system design. The proposal 
includes the design application to the TFF cold spot as well as smaller-scale designs for possible application at secondary areas of contamination in the TFF area not affected by the DUSDP.

The DUSDP was very successful in removing gasoline from the upper and lower steam zone aquifers and probably removed a significant amount of contamination from the intervening clay aquitard. ARV and the follow-up steps explained above should lead us to a condition at TFF where all remaining sources of significant contamination have been removed. What will remain is the "polishing" step of getting the site in compliance with the 1-ppb benzene clean water standard for California. With traditional pump-and-treat and vapor extraction, it will still take considerable time to reach $1 \mathrm{ppb}$ of benzene. This process can be accelerated by the use of enhanced biodegradation. Studies are already going on at LLNL to identify and culture indigenous microbes that break down gasoline into more innocuous chemistry. Planning for a final polishing step using biodegradation is in progress and should be considered as a costeffective means to reach final closure of the TFF site. 


\section{References}

Buscheck, T. A., and J. J. Nitao (1992), Feasibility of In Situ Stripping of Volatile Organic Compounds at the Savannah River Site: Preliminary Modeling of a Pair of Horizontal Wells, Lawrence Livermore National Laboratory, Livermore, CA (UCRL-LR-104540).

Carslaw, H. S., and J. C. Jaeger (1959), Conduction of Heat in Solids, Oxford University Press.

Cook, G. E., J. A. Oberdorfer, and S. P. Orloff (1992), Remediation of a Gasoline Spill by Soil Vapor Extraction, Lawrence Livermore National Laboratory, Livermore, CA (UCRL-JC108064).

Devany, B. (1993), Gasoline volume estimates, Memorandum to Bill McConachie, August 31, 1993, Weiss Associates.

Johnson, P. C., M. W. Kemblowski, and J. D. Colthart (1990), "Quantitative Analysis for the Cleanup of Hydrocarbon-Contaminated Soils by In-Situ Soil Venting," Ground Water, 28, 3, 413-429.

Lee, K. H., A. Kulshrestha, and J. J. Nitao (1993), Interim Report on Verification and Benchmark Testing of the NUFT Computer Code, Lawrence Livermore National Laboratory, Livermore, CA (UCRL-ID-113521).

Newmark, R. (Ed.), (1994), Dynamic Underground Stripping Project LLNL Gasoline Spill Demonstration Report, Lawrence Livermore National Laboratory, Livermore, CA (UCRLID-116964). 


\section{List of Acronyms}

ACWP actual cost of work performed

ARV Accelerated Removal and Validation

BAAQMD Bay Area Air Quality Management District

BCWS Budgeted Cost of Work Scheduled

BTEX benzene, toluene, ethyl benzene, and total xylenes

CoC Chain-of-Custody

CRS Continental Recovery Systems

DUSDP Dynamic Underground Stripping Demonstration Project

ELCD electrolytic conductivity detector

EPA U.S. Environmental Protection Agency

ERD Environmental Restoration Division

ERT electrical resistance tomography

ES\&H Environmental Safety and Health

GAC granular activated carbon

GC gas chromatograph

GC/MS gas chromatograph/mass spectrometer

GSA Gasoline Spill Area

HC hydrocarbons

ICE internal combustion engine

ISTD internal standard

LDV low dead volume injector

LLNL Lawrence Livermore National Laboratory

LOD limit of detection

LSZ lower steam zone

$\mathrm{mg} / \mathrm{L} \quad$ milligrams per liter

MS Matrix Spike

MSD Matrix Spike Duplicate

NPDES National Pollutant Discharge Elimination System

NUFT Nonisothermal Unsaturated-Saturated Flow and Transport

OVA organic vapor analyzer

OVM organic vapor meter

OWS oil/water separator 


$\begin{array}{ll}\text { pH } & \text { puissance d'Hydrogène (hydrogen potential) } \\ \text { PID } & \text { photoionization detector } \\ \text { ppmv } & \text { parts per million by volume } \\ \text { Pvp } & \text { pressure and vapor pressure } \\ \text { QAP } & \text { Quality Assurance Plan } \\ \text { RSD\% } & \text { Relative Standard Deviation Percent } \\ \text { SOP } & \text { Standard Operating Procedure } \\ \text { STP } & \text { Standard Temperature and Pressure } \\ \text { TFF } & \text { Treatment Facility F } \\ \text { TH } & \text { total hydrocarbons } \\ \text { TPH } & \text { total petroleum hydrocarbons } \\ \text { USZ } & \text { upper steam zone } \\ \text { VAC } & \text { volts AC (alternating current) } \\ \text { VOA } & \text { volatile organic analysis } \\ \text { VOC } & \text { volatile organic compound }\end{array}$




\section{Glossary}

Accuracy -- Combination of bias and precision of an analytic procedure, which reflects the closeness of a measured value to a true value.

Bias -- Consistent deviation of measured values from the true value, caused by systematic errors in a procedure.

Calibration standard - A solution prepared from the primary dilution standard solution and stock standard solutions of the internal standards and surrogate analytes. The calibration standard solutions are used to calibrate the instrument response with respect to analyte concentration.

Calibration check standard - Standard used to determine the state of calibration of an instrument between periodic recalibrations.

Detection limit - The lowest concentration level that can be determined to be statistically different from a blank.

Duplicate - Usually, the smallest number of replicates (two), but here specifically refers to duplicate samples, i.e., two samples taken at the same time from one location.

Field duplicates - Two separate samples collected at the same time, placed under identical conditions, and treated exactly the same throughout field and laboratory procedures. Analyses of field duplicates give a measure of the precision associated with sample collection, preservation, and storage, as well as with laboratory procedures.

Field reagent blank - Reagent water placed in a sample container in the laboratory and treated as a sample in all respects, including exposure to sampling site conditions, storage, preservation, and all analytic procedures. The purpose of the field reagent blank is to determine if method analytes or other interferences are present in the field environment.

Internal standard - A pure compound added to a solution in known amounts and used to measure the relative responses of other method compounds and surrogates that are components of the same solution.

Laboratory duplicates - Two sample aliquots taken in the analytic laboratory and analyzed separately with identical procedures. Analysis of laboratory duplicates gives a measure of the precision associated with laboratory procedures, but not with sample collection, preservation, or storage procedures.

Laboratory-fortified blank - An aliquot of reagent water to which known quantities of the method analytes are added in the laboratory. The laboratory-fortified blank is analyzed exactly like a sample, and its purpose is to determine whether the methodology is in control, and whether the laboratory is capable of making accurate and precise measurements at the required method detection limit.

Laboratory-fortified sample matrix - An aliquot of an environmental sample to which known quantities of the method analytes are added in the laboratory. The laboratory-fortified sample matrix is analyzed exactly like a sample. Its purpose is to determine whether the sample matrix contributes bias to the analytic results. The background concentrations of the analytes in the sample matrix must be determined in a separate aliquot, and the measured values in the laboratory-fortified sample matrix must be corrected for background concentrations. 
Laboratory performance check solution - A solution of method analytes, surrogate compounds, and internal standards used to evaluate the performance of the instrument system with respect to a defined set of method criteria.

Laboratory reagent blank - An aliquot of reagent water that is treated exactly as a sample, including exposure to all glassware, equipment, solvents, reagents, internal standards, and surrogates that are used with other samples. The laboratory reagent blank is used to determine if method analytes or other interferences are present in the laboratory environment, the reagents, or the apparatus.

Method detection limit - The lowest concentration of analyte that a method can detect reliably in either a sample or blank.

Precision - Measure of the degree of agreement among replicate analyses of a sample, usually expressed as the standard deviation.

Primary dilution standard solution - A solution of several analytes prepared in the laboratory from stock standard solutions and diluted as needed to prepare calibration solutions and other needed analyte solutions.

Quality assessment - Procedure for determining the quality of laboratory measurements by use of data from internal and external quality control measures.

Quality assurance - A definitive plan for laboratory operation that specifies the measures used to produce data of known precision and bias.

Quality control sample - A sample matrix containing method analytes or a solution of method analytes in a water-miscible solvent that is used to fortify reagent water or environmental samples. The quality control sample is obtained from a source external to the laboratory and is used to check laboratory performance with externally prepared test materials.

Stock standard solution - A concentrated solution containing a single certified standard that is a method analyte, or a concentrated solution of a single analyte prepared in the laboratory with an assayed reference compound. Stock standard solutions are used to prepare primary dilution standards.

Surrogate standard - A pure standard, which is extremely unlikely to be found in any sample, and which is added to a sample aliquot in known amount just before processing so that the overall efficiency of a method can be determined. 
Appendices 


\title{
Appendix 1
}

\section{Accelerated Removal and Validation Project (ARVP) Management}

\author{
(Alan B. Copeland, Environmental Restoration Division, LLNL)
}

\section{Project Development}

This work was sponsored by EM-44 in the form of a \$1M supplement to the LLNL Environmental Restoration Division budget. This was made available in the September 1993 Financial Plan.

This funding came in response to an informal proposal dated August 3, 1993. The proposal contained three parts. Part 1 was to execute 3 months of continuous gasoline removal operations to take advantage of the elevated temperature of the site. Included in this part was further automation of the systems at TFF to reduce operating expenses, and temperature monitoring. The cost of this part was estimated to be $\$ 900 \mathrm{~K}$. Part 2 was for additional characterization of the TFF site by boring 12 additional wells and analyzing the cores taken from them. This, with well completions, was estimated to cost $\$ 900 \mathrm{~K}$. Part 3 was to do additional electrical or steam heating as indicated by the results of the additional characterization of the site in part 2 . This was estimated to cost $\$ 2.5 \mathrm{M}$.

The proposed efforts were rescoped to fit the dollars made available, and the resulting plan, in the form of a critical path network, is shown in Figure A1-1. Part 1 of the proposal was included with a limited amount of automation planned. Part 2, site characterization, was not included. From part 3 of the proposal, we included preparation for using the four new wells that had been completed at the end of the second phase of Dynamic Stripping in a refurbished electrical heating network to heat the known cold spot in the center of the site.

In addition to the above, we planned to develop a computer model of the electrical heating and some geochemical properties measurements for use in the model. A brief air sparging campaign, designed to be very low in cost, was included to provide a basis for future work.

\section{Project Team}

The project team organization and principal participants are shown in Figure A1-2. Continuity with the work done during the Dynamic Stripping phases was provided by including key scientific, engineering, and technical personnel involved in those phases on the team. 


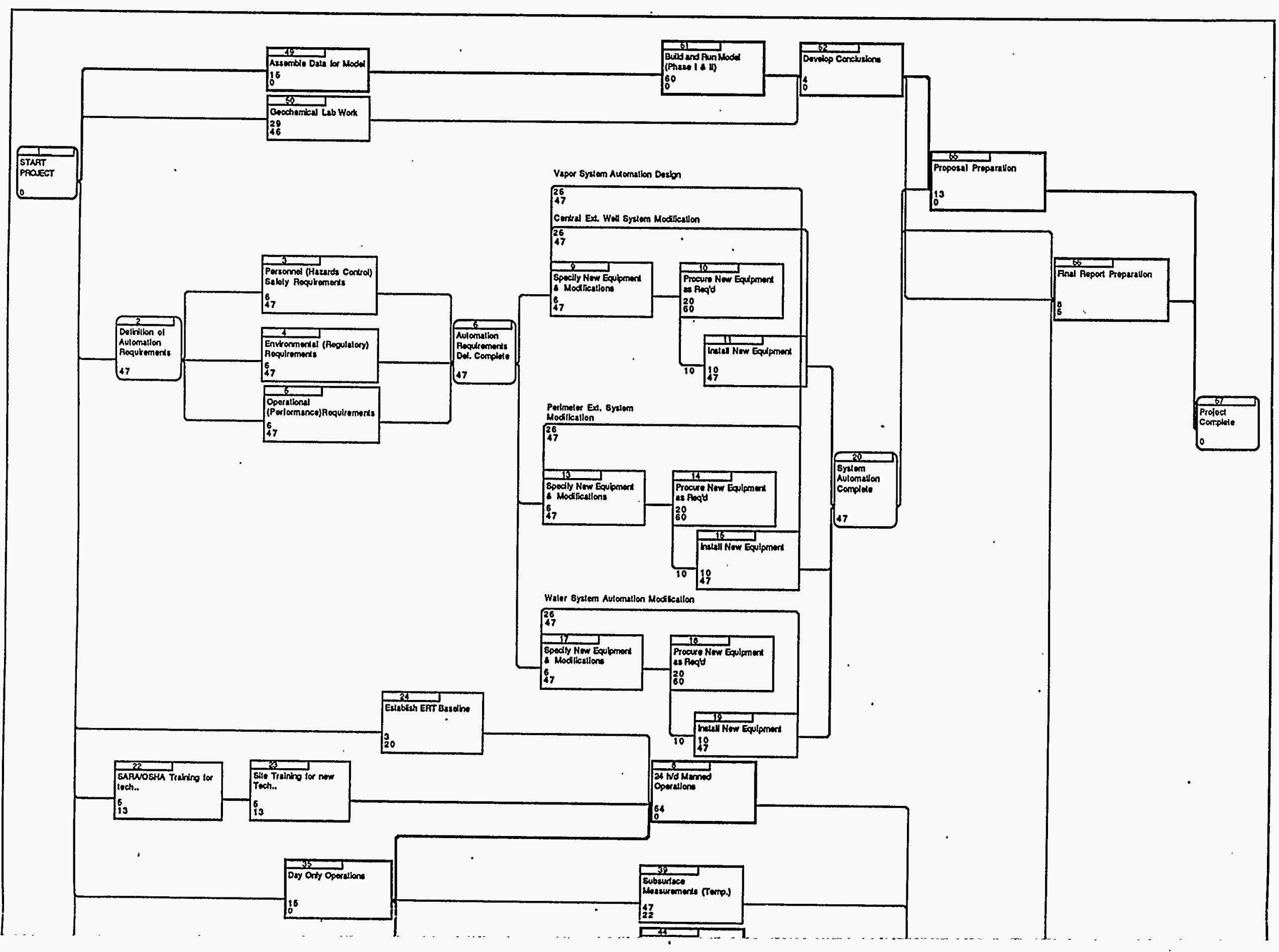




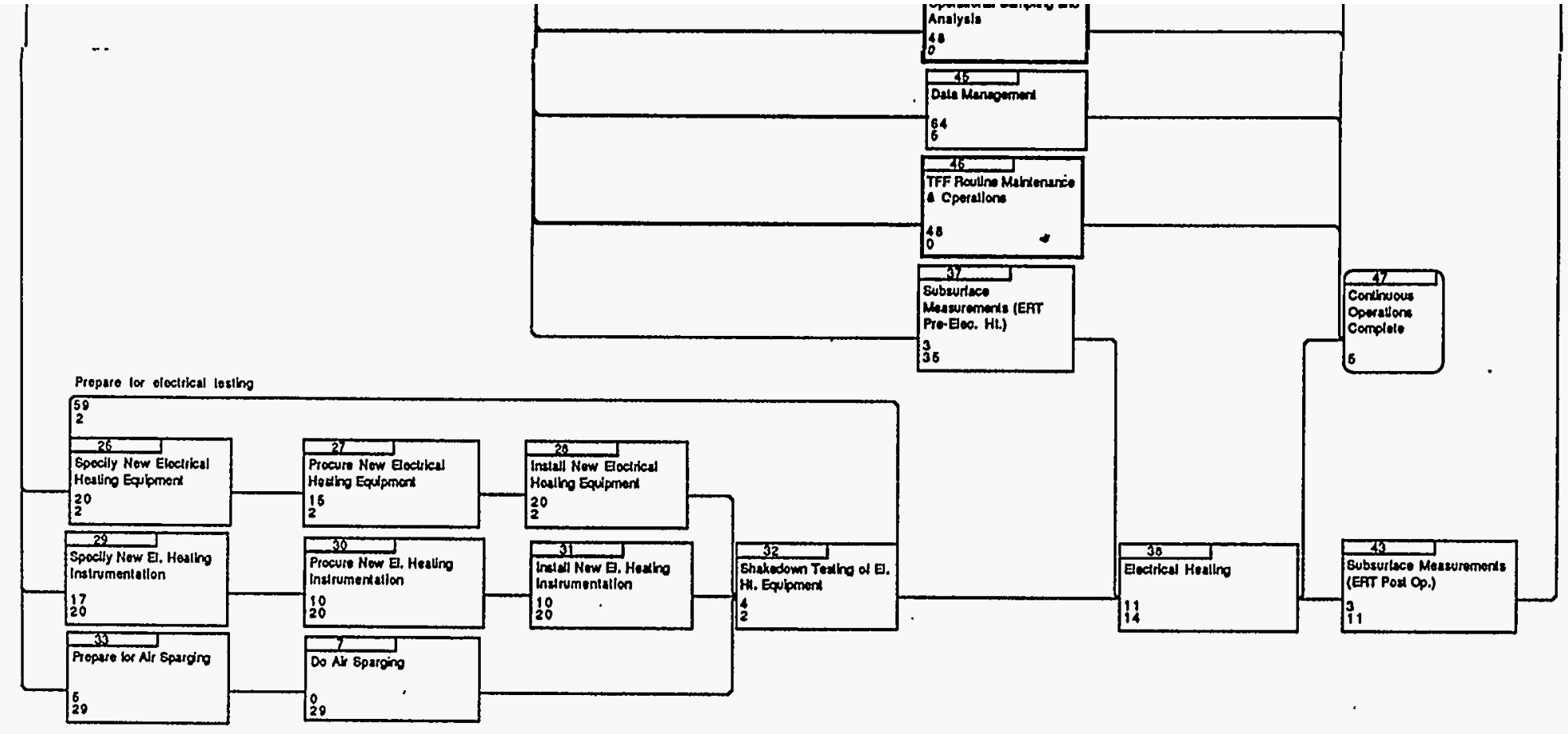

Figure Al-1. Critical path network for Accelerated Removal and Validation Project. 


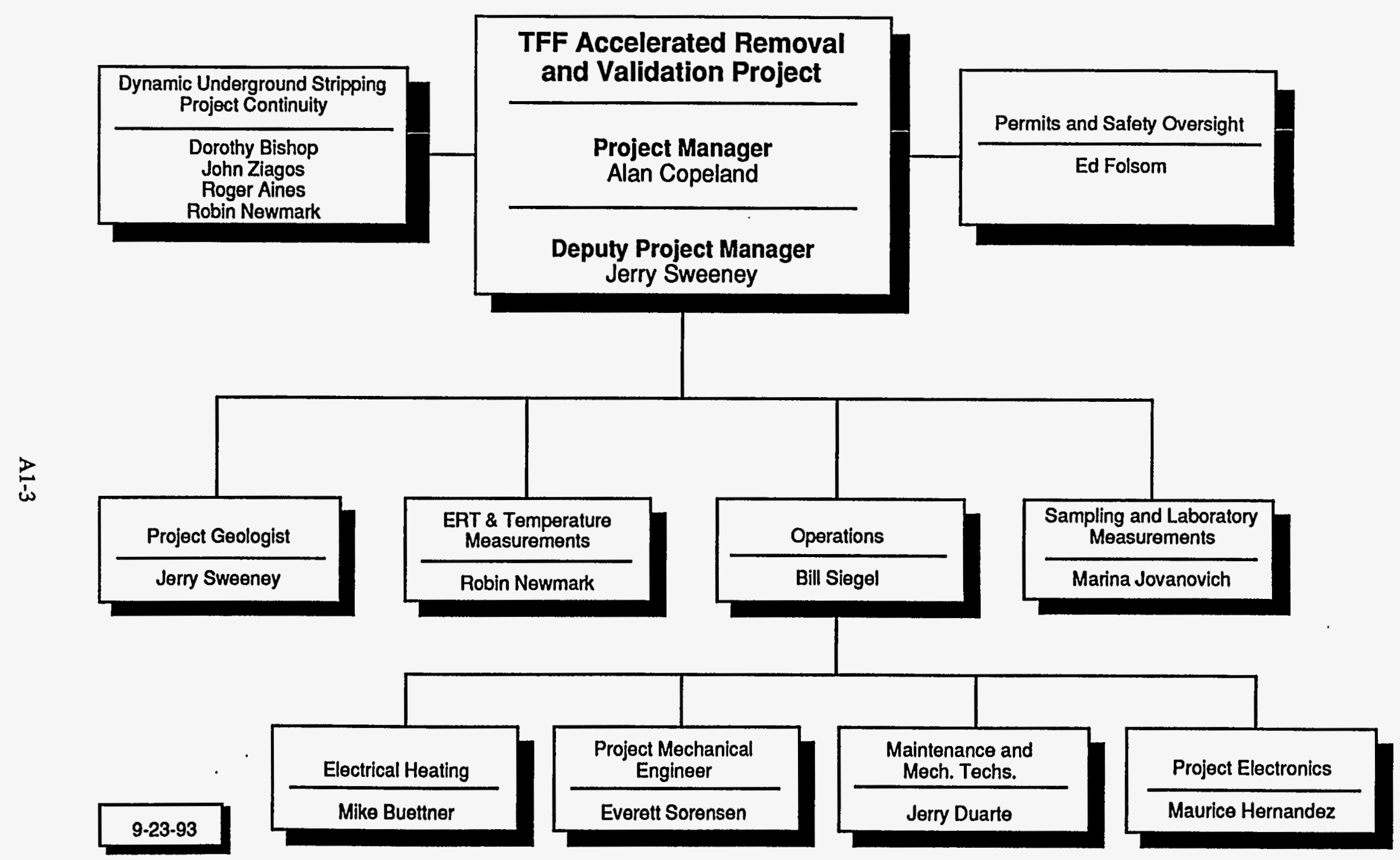

Figure A1-2. Organization chart showing project team and principal participants. 


\section{Project Execution}

A timeline for project activities is shown in Table A1-1. As can be seen, both the air sparging and electrical heating operations were of very limited duration. This was due to the constraints of budget and the stipulation that these supplemental funds be expended prior to the end of the first quarter of FY94.

A plot of the project management parameters: Budgeted Cost of Work Scheduled (BCWS); Budgeted Cost of Work Performed (BCWP); and Actual Cost of Work Performed (ACWP) is given in Figure A1-3. The initial rate of accomplishment was higher than expected in the first 2 months. Overall, the project was completed on time and for the budget allocated. Some work done during the time of this project, which was related to the ongoing operations of TFF, was funded from the normal operating budget for the Livermore Site remediation. This averaged $\$ 120 \mathrm{~K} /$ month for September, October, and November.

A breakdown of the ARVP expenditure by project area is given in Figure A1-4. Preparations for electrical heating and 2 weeks of heating cost approximately $\$ 100 \mathrm{~K}$ more than was planned. The main reasons for this were unplanned scope of work in refurbishing the temperature logging systems, and the establishment of full displays and control of the pumping and treatment system operation in the control trailer for remote operation during electrical heating. As a result, the amount of general system automation completed to allow unmanned operation was less than originally expected. Also, offsetting the unplanned electrical heating expenditures were lowerthan-expected operations costs from the use of adjusted workweeks instead of overtime for people covering the weekend operations.

\section{Conclusions}

The primary objectives as set out in the project plan were achieved. The time constraint of 4 months forced some project activities to be in parallel, which given more time would better have been done in series. For example, the computer modeling of both air sparging and electrical heating, had it been completed before the experimental work, might have altered the experiment plans for both. Also, more time to pursue the air sparging possibilities, while set up to do that, could have given more return for not much additional cost. 
Table A1-1. ARV project timeline.

\begin{tabular}{|c|c|c|}
\hline Date & Time & Activities \\
\hline $10 / 04 / 93$ & 12:20 p.m. & Facility on line \\
\hline $10 / 18 / 93$ & 10:30 a.m. & Vapor extraction stopped from GEWs-808 and 816 \\
\hline 10/18/93 & 11:00 a.m. & Vapor extraction started from GIWs-813, 815, 818, and 820 \\
\hline $10 / 26 / 93$ & - & GSW-16 isolated from vapor extraction system \\
\hline $11 / 02 / 93$ & $3: 45$ p.m. & GIW-813 taken off-line \\
\hline $11 / 04 / 93$ & - & GIW-813 back on-line \\
\hline $11 / 05 / 93$ & - & Entire system shut down-power outage over weekend \\
\hline 11/08/93 & 11:00 a.m. & Facility restarted \\
\hline $11 / 09 / 93$ & - & $\begin{array}{l}\text { Vapor extraction started from GSW-16, GEW-816, and GEW-808, vapor } \\
\text { extraction stopped from GIWs-813, 815, 818, and } 820\end{array}$ \\
\hline $11 / 16 / 93$ & 8:40 a.m. & Air sparging experiment started \\
\hline 11/16/93 & 11:00 a.m. & $100 \%$ tracer recovery in GIW-815, $2 \%$ tracer at ICE \\
\hline $11 / 16 / 93$ & $3: 00$ p.m. & End of first air sparging test \\
\hline 11/18/93 & - & Extracting vapor from GIW-820 only \\
\hline 11/21/93 & - & Electrical heating test run, voltages measured \\
\hline $11 / 22 / 93$ & 11:00 a.m. & Air sparging restarted in GIW-815 \\
\hline $11 / 22 / 93$ & $3: 30$ p.m. & Stopped extracting from GIW-820 \\
\hline $11 / 22 / 93$ & 4:45 to $7: 45$ p.m. & Electrical heating system test \\
\hline $11 / 22 / 93$ & 7:00 p.m. & Resumed extracting vapor from GEWs-808 and 816 \\
\hline $11 / 24 / 93$ & - & Facility shut down for the holidays \\
\hline \multirow[t]{2}{*}{$11 / 29 / 93$} & $8: 00$ a.m. & Ground water system restarted \\
\hline & 12:45 p.m. & IC engine restarted \\
\hline $11 / 30 / 93$ & 7:00 p.m. & Electrical heating started \\
\hline $11 / 30 / 93$ & $8: 00$ p.m. & Power outage, all systems down \\
\hline $12 / 01 / 93$ & 6:30 a.m. & Electrical heating off \\
\hline $12 / 02 / 93$ & 11:00 a.m. & $\begin{array}{l}\text { Extracting vapor from the three central extraction wells (GSW-16 block } \\
\text { removed) }\end{array}$ \\
\hline $12 / 03 / 93$ & 6:10 p.m. & Electrical heating system operated overnight \\
\hline $12 / 07 / 93$ & 5:00 p.m. & Electrical heating system operated overnight \\
\hline 12/08/93 & 4:55 p.m. & Electrical heating system operated overnight \\
\hline $12 / 09 / 93$ & $5: 00$ p.m. & Electrical heating system operated overnight \\
\hline $12 / 10 / 93$ & 5:00 p.m. & Electrical heating system operated overnight \\
\hline $12 / 12 / 93$ & 9:00 p.m. & Extracting vapor from GIW-814, GIW-815, HW-1 \& 2 \\
\hline $12 / 13 / 93$ & - & ARV 24 h/day phase completed \\
\hline
\end{tabular}




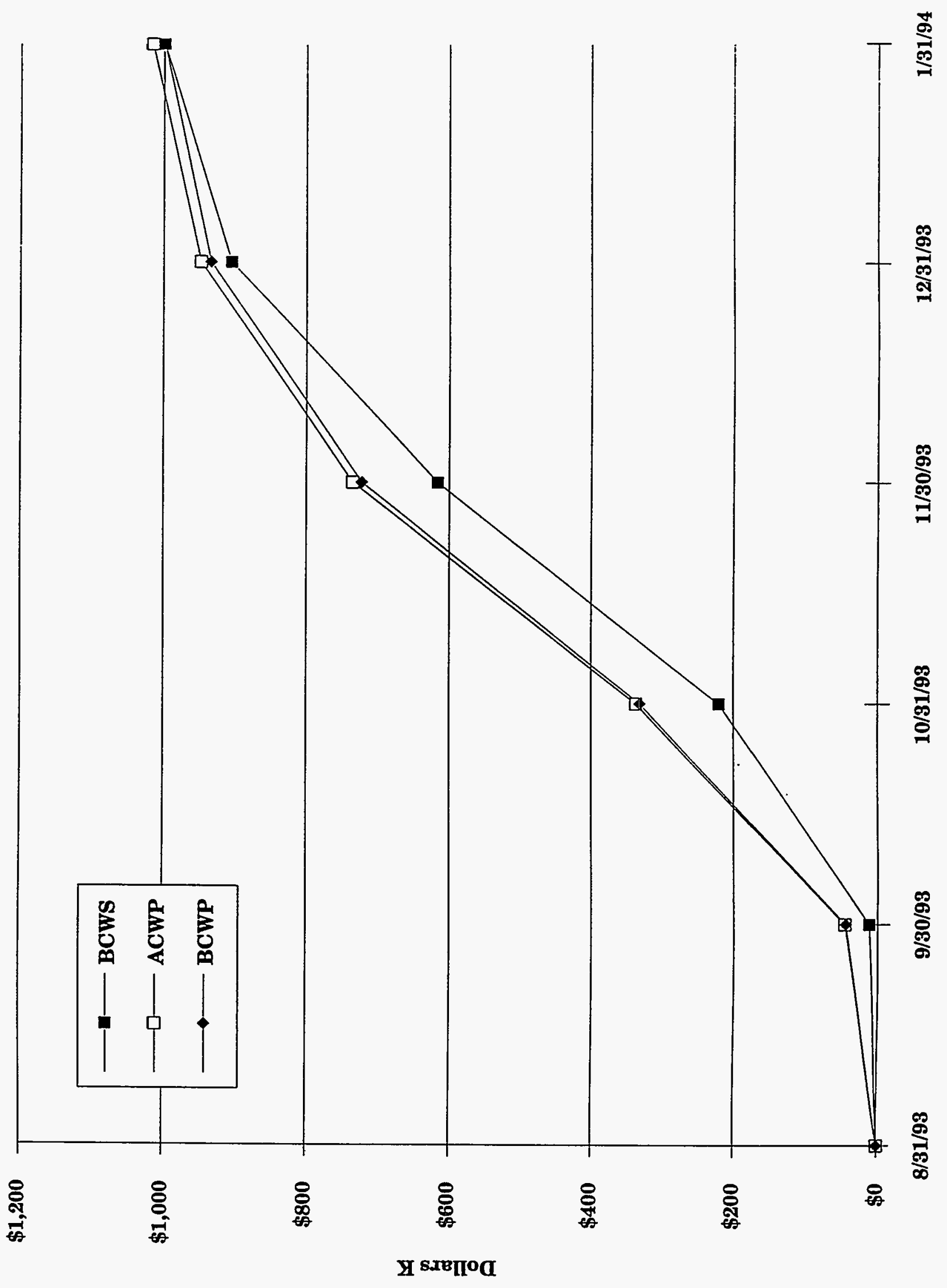




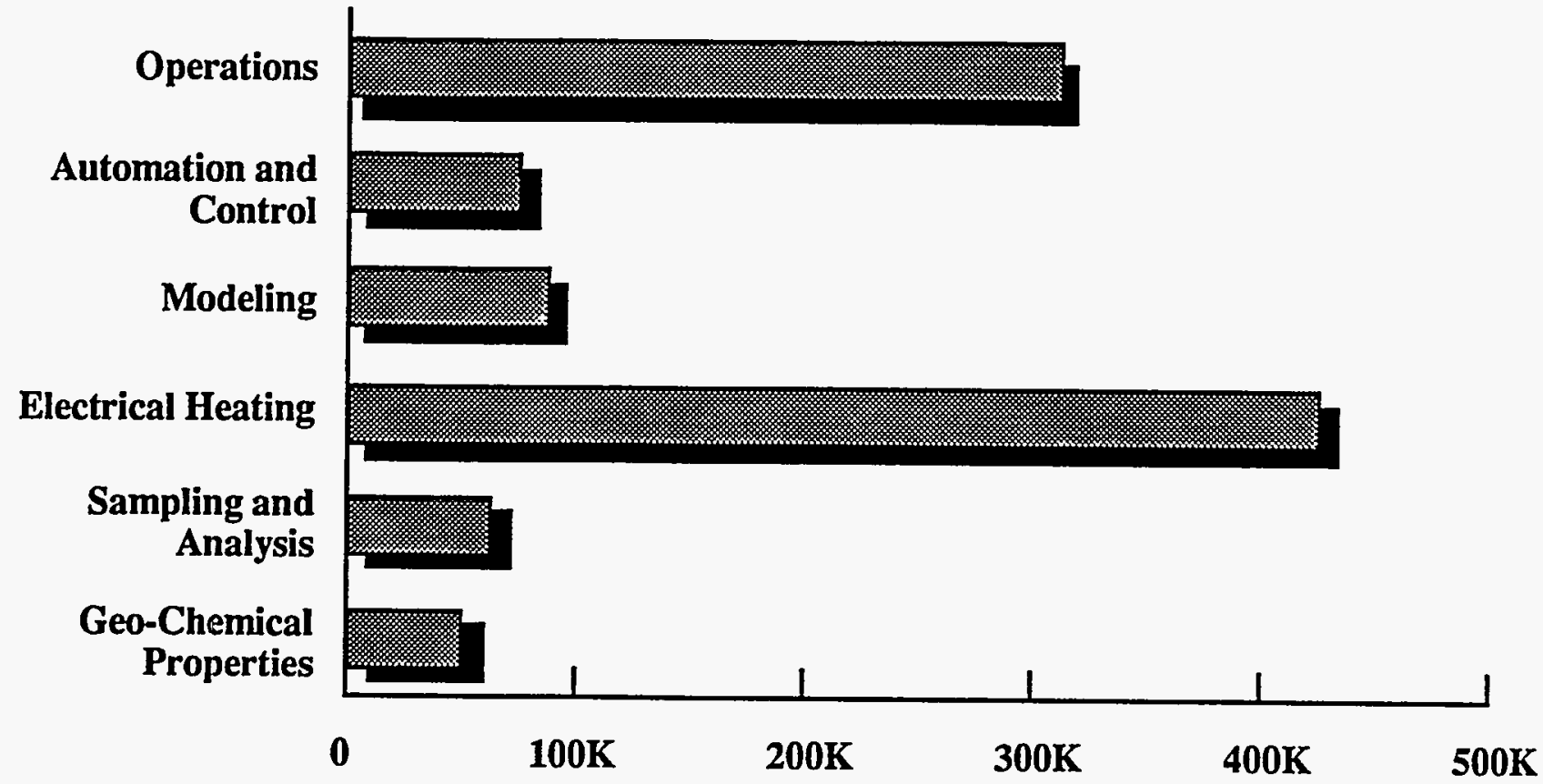

Figure A1-4. ARV project expenditures by major activity. 


\section{Appendix 2}

\section{System Automation}

\section{(Maurice Hernandez, Defense Science Engineering Division, LLNL)}

This Appendix describes the present status of system automation efforts for TFF. This includes a brief historical design perspective and summary descriptions of the various automated control components. These are followed by recommended approaches to reaching the objective of safe, reliable automatic system operation.

\section{Background and History}

In 1988, TFF was constructed for an EPA-approved pilot study to evaluate vacuum-induced venting and treatment of FHC vapor. Vacuum-induced venting tests were conducted through 1991. The facility was subsequently modified for the DUSDP. When the Record of Decision was signed in August 1992, TFF was converted from pilot study to Remedial Action status.

Amidst these changes in the scope of TFF operations, we designed the main facility piping and instrumentation in early 1992, with construction taking place from June 1992 through January 1993. DUSDP modifications were designed and constructed between mid-1992 and October 1993. The activation phase of the main facility occurred between November 1992 and January 1993.

Due to the variances in operational scope and schedule acceleration brought on by the necessity for meeting regulatory milestones, a critical path approach was undertaken during the latter stages of the facility implementation phase. The idea behind this approach was to bring the system components on line as quickly as possible with manual control, while making provisions for a phased implementation of the automatic controls. Subsequently, budget and manpower constraints, as well as shifting priorities, have left TFF with the intended automatic capabilities only partially implemented. More information on the present status is given in the next sections.

\section{Control System Status}

Status is presented below for the various categories of the automatic control system.

\section{Computers and Software}

A 486-based personal computer (PC) with Genesis application software is presently in place at TFF. Genesis is a commercial software package designed for creating control system applications. The present software provides for a large percentage of the facility control, monitoring, and interlock needs. However, the implementation is best described as rudimentary, and some issues remain. These include long-term code maintenance, design documentation, code 
verification and validation, flexibility for future modifications, and standardization with other ERD treatment facilities.

The operator software interface is identified as one further significant shortcoming. While nearly all pertinent information and control functions are available to the operator, it is not well presented. Graphic and functional limitations, ambiguous colors and symbols, and poor layout have made the interface nonintuitive and mistake prone. This also leads to significant training and retraining costs.

\section{Instrumentation}

The facility has been fully instrumented as specified by Remedial Design Report No. 2 (RD2). However, a more recently identified requirement (total effluent flow) has not been implemented, and several existing components must be repaired or modified. Examples include some that display erroneous or spurious readings (oil content monitor, LVB-60 influent flow, several thermocouples, demister effluent air flow), damaged sensors (GEW-816 well level transducer), and an improperly wired status/alarm panel. A comprehensive re-assessment of the control requirements may also identify additional instrumentation needs.

\section{Safety and Interlocks}

In general, the primary identified safety and failure mode considerations have been addressed, and there are no safety issues for manual operation. However, several interlocks that would be required for unmanned operation are routinely bypassed during manned operations due to uncertain performance and spurious false shutdowns. To date, a formal failure mode analysis for unmanned, standalone operation has not been carried out. Cursory reviews have found that the present interlock system design is not inherently fail-safe, as dictated by the standalone design requirements. Other issues such as adequately meeting requirements for intrinsically safe installations, and facility electrical bonding and grounding have been reviewed for manned operation only.

\section{Quality Control and Quality Assurance}

Quality Control and Quality Assurance requirements for the facility are defined in the Remedial Action Implementation Plan (RAIP), RD2, and the ERD Quality Assurance Plan (QAP). Certain aspects of these are addressed in the present TFF design; however, these (system testing, document control, process monitoring) are carried out in a less formal manner than required for unmanned operation. Other requirements (QA/QC tracking, design control and changes, operating procedures, failure root cause analyses, and equipment calibrations) are not well established. Overall, a more formal approach is required for unmanned operation.

\section{Recommendations}

The following are recommendations to achieve a fully automated facility. 


\section{General}

The overall facility control and monitoring requirements should be re-evaluated with respect to the present configuration and any foreseeable facility modifications. Some of the possible upgrades are identified in the RAIP and RD2. This must result in a formal agreement (i.e., a reviewed and approved document) enumerating the present needs, and providing for flexibility to implement future modifications. This document will also provide criteria against which ensuing designs and modifications are reviewed, and, as such, is subject to formal change control.

\section{Computers and Software}

Control software should be designed to meet the formal, approved requirements, using up-todate analysis, modeling, and review techniques. This will provide assurances that design criteria are met, as well as establishing a design configuration baseline on which future additions and modifications can be based (design control). This will result in greater efficiency in maintenance and upgrades over the life of the facility.

The current Genesis application software should be replaced with the Paragon TNT package. This will standardize TFF with other LLNL treatment facility software, which will facilitate maintenance, and is necessary for networking.

\section{Instrumentation}

The problem areas described above must be resolved. The approved requirements will be used as a basis for the updated instrumentation design. It will likely also identify previously overlooked instrumentation needs, and perhaps eliminate some that are no longer applicable. The result will be a more comprehensive approach and a solid, robust design.

\section{Safety and Interlocks}

A comprehensive failure mode analysis must be done. The interlocks will then be designed to meet the identified needs and rigorously reviewed. Special emphasis will be placed on fail-safe implementation and provisions for operational issues, such as set points, alarms, and safe design of only absolutely necessary bypass mechanisms. Where possible, bypass mechanisms will be avoided. All identified special circumstances requiring bypass of normal interlocks will be implemented using specifically designed operational modes.

Installations will be thoroughly evaluated for intrinsic safety where required. Necessary modifications for strict adherence should be immediately undertaken.

\section{Quality Control and Quality Assurance}

Procedures must be put in place to meet the requirements of the RAIP, RD2, and ERD QAP. Care should be taken to meet the spirit and intent of the requirements, while not placing an unreasonable burden on facility personnel. Particular areas of concern are design documentation and control, design and modification reviews, and periodic inspections and maintenance. 


\section{Conclusions}

Treatment Facility $\mathrm{F}$ requires a significant effort to reach true automated capabilities. A preliminary estimate totals $\$ 330 \mathrm{~K}$ ( $\$ 290 \mathrm{~K}$ effort, $\$ 40 \mathrm{~K}$ equipment). While the costs incurred to make this effort are significant, the long-term gains should merit the expense. 


\title{
Appendix 3
}

\section{Electrical Heating Systems}

\author{
(Ray McNairy, Defense Sciences Engineering Division, LLNL)
}

\section{Instrumentation and Control}

We developed instrumentation systems to monitor heater electrodes and soil temperatures for the ARV phase. The implemented system was designed to acquire real time data in a highvoltage, common mode environment where the electrode drive potential could appear at the lowvoltage input of the instrumentation electronics. The electrode drive potential compromised the operation of the original data acquisition system. For this reason, we redesigned the entire system.

The heating system design required that $60 \mathrm{~Hz}$ power be applied to up to nine heating electrodes buried in the earth. The system had to handle electrode drive voltage levels of up to $600 \mathrm{VAC}$ with current levels to $600 \mathrm{~A}$. The system also had to record data from thermocouples grounded to the heating electrodes at discrete levels, thermocouples placed in nonheated "soil wells," as well as electrode drive current plus monitoring miscellaneous transducers as required.

Because of the maximum of $600-\mathrm{VAC}$ electrode drive potential, the challenge was to reliably record millivolt-level thermocouple signals at a $600-\mathrm{VAC}$ common mode potential. This common mode potential results from the thermocouple leads being grounded to the measured electrodes, thus exposing the thermocouples to the drive potential. In the new system design, we force the high common mode potential to appear across the instrumentation input power. This allows the electronics to be driven at the common mode potential of the individual electrode, thereby providing a low reference voltage for the system to operate against. The electronic system chosen was a commercially available OPTO-22 digital system controlled by Labview software. The OPTO-22 configuration was chosen for expediency because of the short development and fielding time allowed, and its successful use in past experiments. The OPTO22 implementation was successful.

The OPTO-22 components are a modular instrumentation concept developed to serve the needs of the industrial process control community. As such, the system is extremely flexible and can condition and record large quantities of data at less than 10 channels/sec rates. Additionally, optical isolation is built in at the individual channel level; this allows a large latitude in transducer interconnection and configuration capability.

As configured (Figs. A3-1 and A3-2), each heating electrode has a dedicated OPTO-22 electronic front end for analog to digital conversion, scaling, and digital formatting of the millivolt level thermocouple data. Data communications to the individual electronics systems are accomplished with bidirectional digital fiber optic links to a control and archiving Mac II 


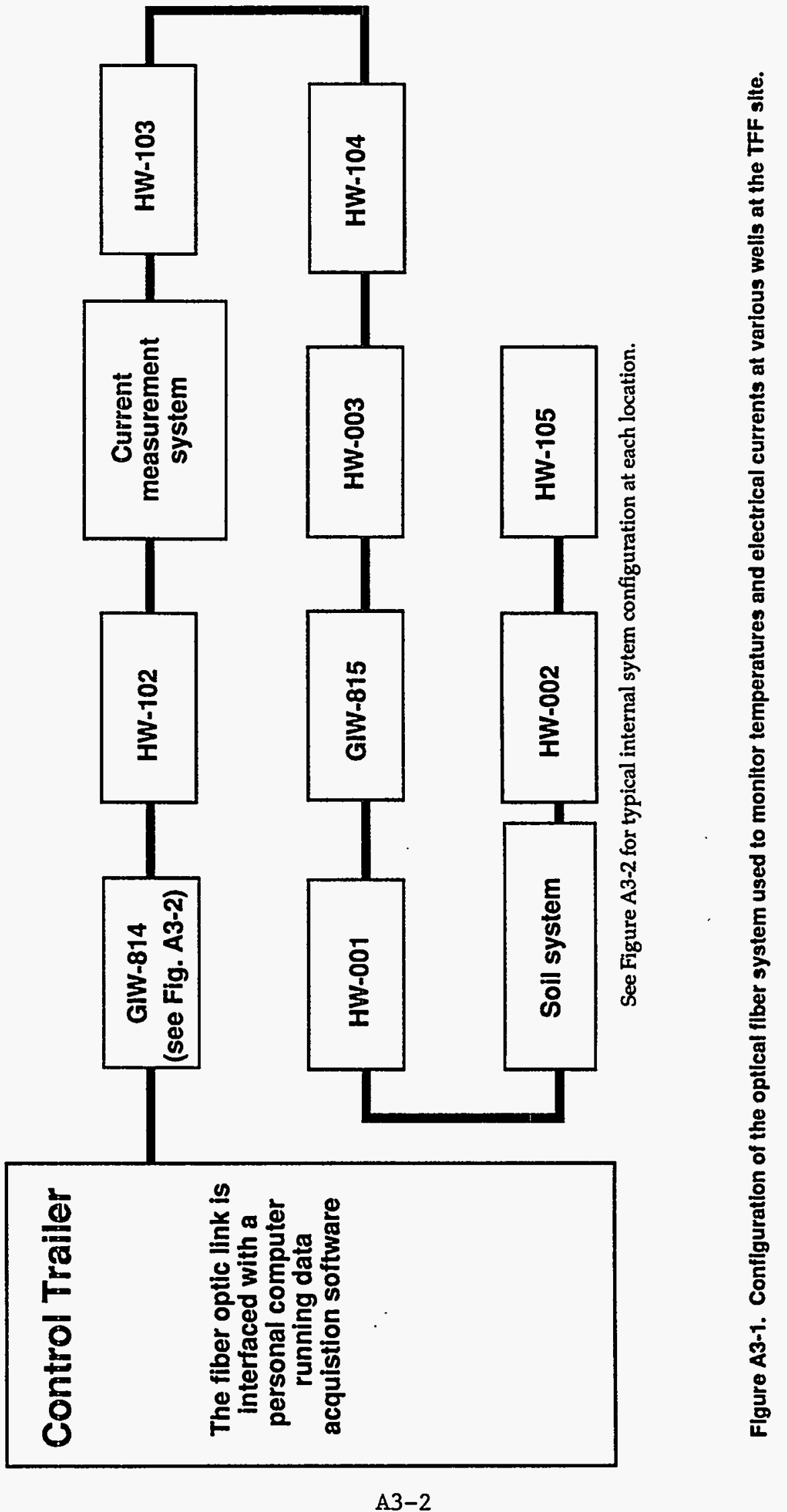




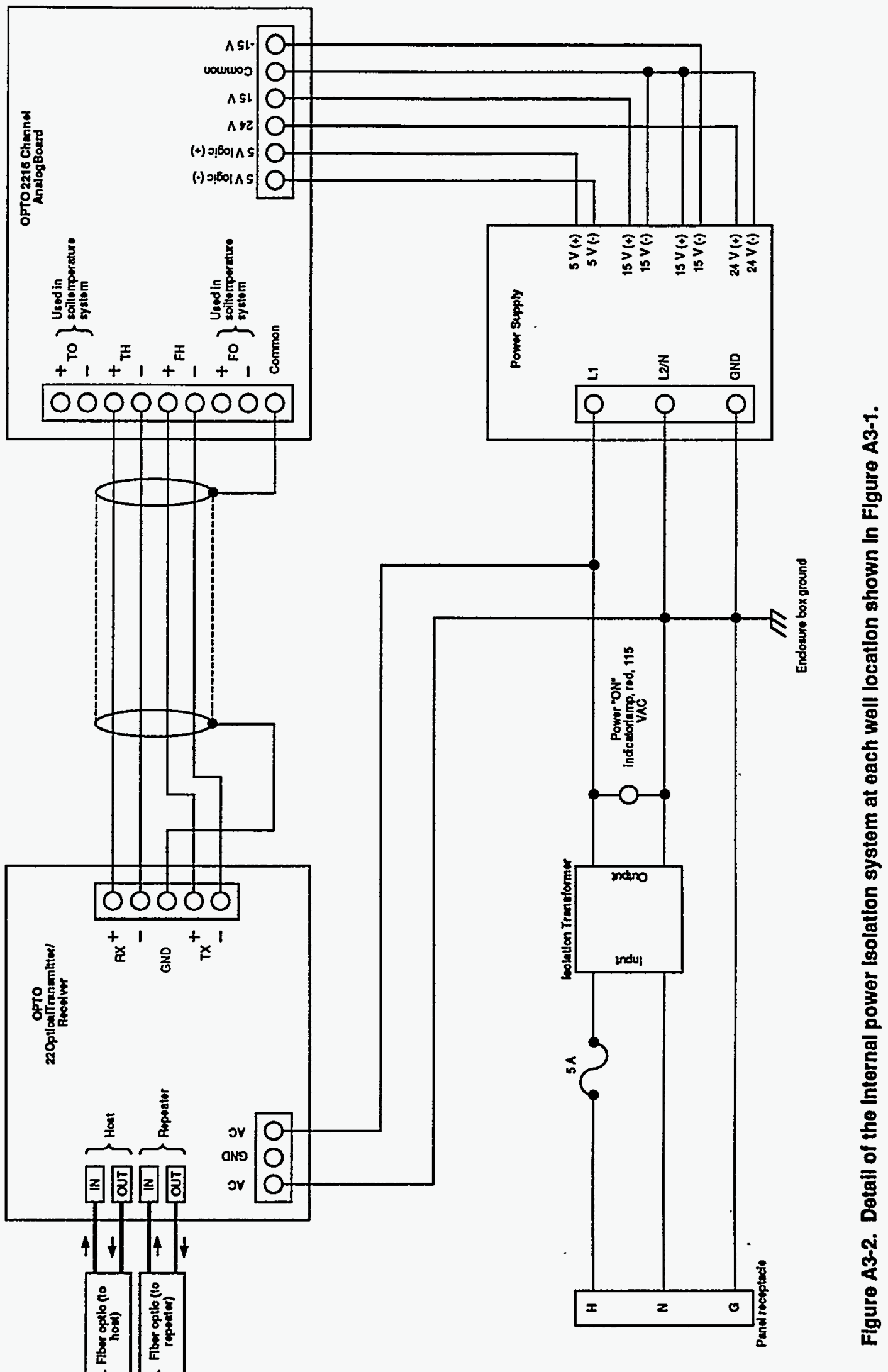


computer in the control trailer. The links are in a "daisy chain" configuration and conform to an industry standard data protocol, RS-488. The optical data link portion is a component of the OPTO systems concept.

Soil thermocouples are recorded by an additional, larger, OPTO-22 system of similar configuration to that above. Because the soil measurement electrodes are not exposed to the high common mode voltages of the heating electrodes, multiple measurement locations were successfully recorded with a common system. The soil thermocouple was controlled via the same fiber optic link as the heating electrode units and the unit for the electrode current measurements.

Grounding the OPTO-22 chassis and the fiber optic link to the electrode is analogous to placing the systems in a "bottle" that then can operate independently of electrode drive potential up to the design level of $600 \mathrm{VAC}$. The instrumentation system performed as designed after minor startup problems and proved quite reliable during the active heating phase of the experiment.

The heating electrode instrumentation configuration has a 16-channel capacity. Only four of these channels are populated with the analog input modules required to condition the two types of thermocouples installed. The electrode current system is identical to the heating electrode system except that 12 channels are used to acquire data from the 12 electrode current measurements. The soil system is an expansion of the base line heating well system and incorporated a 6 by 16 channel capability, of which 80 channels were populated. Low-voltage power supplies were installed at each measurement location. These identical supplies provide additional flexibility and power isolation, if needed. Primary isolation is provided by an isolation transformer and power connectors, rated for 600 VAC operation but operated at 115 VAC levels. The power configuration conforms to the National Electrical Code and was reviewed and approved for personnel safety both for in situ operations and during bench checkout. When the heating electrodes are not operating, the safety ground for the system is provided by the system chassis connection to the electrodes, which are earth referenced. In addition, a ground wire is installed in all power input cables, except the heating well systems, for a safety chassis ground. During heating, safety was controlled by rigid administrative control and site access control procedures. We used Operational Safety Procedures that were developed and carried over from the original heating experiment. 


\section{Power System}

\section{(H. Michael Buettner, Engineering Research Division, LLNL)}

The electrical power system used for ARV was the same one used for the DUSDP (with some modifications) and is described below. When DUSDP concluded, some parts of the electrical power system were removed. In particular, the main circuit breaker, which had been leased, was returned to the vendor. Also, some of the wiring was cut to expedite removal.

Before ARV began, we had to obtain another main circuit breaker, splice some of the wiring, and reroute some of the existing power cables to the new heating wells.

The electrical heating system derives its power from the LLNL grid. A block diagram of the power distribution system is shown in Figure A3-3. Power is supplied from the grid at $13.8 \mathrm{kV}$ and applied to the primary side of a 1,500 KVA transformer by means of a $15-\mathrm{kV}, 600-\mathrm{A}$ load interrupter switch. The working voltages on the secondary side of the transformer are 208, 350, 480 , and $600 \mathrm{VAC}, 60 \mathrm{~Hz}$, three-phase. These voltages were chosen to offer some degree of control over the electrode currents. The lowest value was selected to account for the lowest interelectrode resistance we expected to encounter, while the highest value was chosen because it was the highest practical voltage we could use without resorting to special high-voltage equipment and safety requirements.

The secondary voltage is switched ON or OFF by the main circuit breaker $(5,000 \mathrm{~A}$, $600 \mathrm{VAC}, 3$ pole) and transmitted by a wireway to a low-voltage switchboard. The switchboard contains 12 adjustable trip breakers (one per heating electrode), and it is at this switchboard that the secondary voltage and appropriate phase are assigned to each electrode. The detailed specifications of the electrical system may be found in Newmark et al. (1994).

\section{References}

Newmark, R. (Ed.) (1994), Dynamic Underground Stripping Project LLNL Gasoline Spill Demonstration, Lawrence Livermore National Laboratory, Livermore, CA (UCRL-ID116964). 


\section{Electrical Heating Surface Equipment}



Figure A3-3. Diagram of the electric power system supplying power to the heater electrodes. 


\title{
Appendix 4
}

\section{Operational Measurements, Sampling, and Chemical Analyses}

\author{
(Marina C. Jovanovich, Roger E. Martinelli, Health \& Ecological \\ Assessment Division, LLNL - Michael J. Dibley, and Kenneth L. \\ Carroll, Environmental Restoration Division, LLNL - Everett \\ Sorensen, Weiss Associates, Emeryville, CA)
}

This Appendix describes regulatory requirements, flow measurements, and chemical analyses for the ARV project at TFF. Data were collected primarily for measuring contaminant removal, experimental evaluation of the facility's performance under various operating conditions, and regulatory compliance verification.

\section{Regulatory Compliance}

Remediation of LLNL is enforced under CERCLA requirements by the Federal EPA, California EPA, the Bay Area Regional Water Quality Control Board (RWQCB), and others. Day-to-day operations of treatment facilities are regulated by the Bay Area Air Quality Management District (BAAQMD) and the Livermore Water Reclamation Plant (LWRP), which both required LLNL to obtain operating permits for the treatment facility.

To comply with discharge permits issued by the BAAQMD and the LWRP, we measured water and air discharge rates and analyzed these discharges for chemical composition. Table A4-1 summarizes regulatory compliance requirements for TFF.

BAAQMD. Three operations at TFF were permitted by the BAAQMD. These are the ground water treatment system air stripping discharge, the Continental Recovery Systems (CRS) regenerable carbon system, and the internal combustion engine (ICE). Each of these operations is restricted with individual operating conditions. These conditions include hydrocarbon concentration limits in the discharges; mass discharge limits for certain toxic compounds, such as benzene; destruction efficiencies; monitoring requirements; and record keeping.

LWRP. The LWRP is concerned only with the treatment facility's sewer discharge. The treatment facility was originally designed to achieve National Pollutant Discharge Elimination System (NPDES) discharge limits (natural receiving water discharge limits) established by the Federal EPA through the RWQCB. However, certain compounds in extracted ground water were not destroyed by UV/oxidation and were not completely stripped in the final air stripping stage. To operate the treatment facility and complete steam injection by the June 30 air district boiler exemption expiration date, LLNL researchers opted to discharge treated water to the sanitary sewer rather than attempt to improve the facility's performance to meet NPDES limits. 
Table A4-1. Regulatory compliance requirements for LLNL Treatment Facility F.

\section{BAAQMD}

- Facility benzene discharge limit: $1.8 \mathrm{lb} /$ day (confirmed monthly).

- E006-AQ counts towards total benzene discharge.

- Log book maintained daily (hours of operation, analytic results, carbon replacement).

- Source test required for CRS trailer within 30 days of startup.

- Permit posted in B406.

Sampling and Analysis Requirements:

\begin{tabular}{|c|c|c|c|c|}
\hline Sample point & Method & Analyte & Frequency & Discharge limit \\
\hline CFO & OVAFID & TH & $5 /$ week & $10 \mathrm{ppmv}$ \\
\hline E006-VPR (a) & OVAFID & TH & $5 /$ week & $10 \mathrm{ppmv}$ \\
\hline UVO5 & EPA 601/602 & TH/BTEX & $1 /$ month & Not Applicable (b) \\
\hline
\end{tabular}

\section{Livermore Water Reclamation Plant (LWRP)}

- 75 gpm maximum discharge rate, $50 \mathrm{gpm}$ average.

- Permit posted in B406.

Sampling and Analysis Requirements:

\begin{tabular}{|c|c|c|c|c|}
\hline Sample point & Method & Analyte & Frequency & Discharge limit \\
\hline E006-AQ & EPA 624 & BTEX & Quarterly & $250 \mathrm{ppb}$ \\
\hline E006-AQ & EPA 625 & Toxic organics & Annually & $1,000 \mathrm{ppb}$ \\
\hline E006-AQ & EPA 6010 & Metals & Annually & See list below (c) \\
\hline E006-AQ & EPA 335.2 & Cyanide & Annually & $40 \mathrm{ppb}$ \\
\hline
\end{tabular}

Notes:

(a) CRS sampling only after startup.

(b) Air stripper analysis for characterization, no concentration limit.

(c) Metals discharge limits: As: 60 ppb; Cd: 140 ppb; Cu: 1,000 ppb; Total Cr: 620 ppb;

Pb: 200 ppb; Hg: 10 ppb; Ni: 610 ppb; Ag: 200 ppb; Zn:3,000 ppb.

TH $=$ total hydrocarbons.

BTEX = benzene, toluene, ethyl benzene, and total xylenes. 
The LWRP requires quarterly analyses of the sewer discharge. The LWRP discharge concentration limits are considerably higher than NPDES limits and are therefore more easily achieved. Unfortunately, discharging to the sanitary sewer costs about $\$ 7$ per 1,000 gal discharged.

Since contaminant concentrations in extracted ground water have declined significantly since facility start-up, the facility now nearly achieves NPDES discharge limits in treated water. In December 1993, we analyzed the effluent for NPDES-regulated compounds. The only analyte detected above NPDES limits was manganese, detected at $780 \mathrm{ppb}$. The NPDES discharge limit for manganese is $500 \mathrm{ppb}$. Freon and acetone were detected at slightly elevated levels, but were attributed to chromatograph column cleaning solvents.

Current plans include continued NPDES compliance analyses to evaluate natural receiving water discharge options.

\section{Contaminated Removal and Experimental Evaluation}

For experimental purposes, we collected data to evaluate accurately the gasoline removal rate. This included frequent sampling and analyses of vapor and water at several points throughout the system and bi-hourly recording of operational parameters, such as flow rates, applied vacuum, temperatures, pressures, and gasoline accumulation. We analyzed each sample for its contaminant composition.

\section{Ground Water and Vapor Sampling}

During the ARV project, we sampled the aqueous stream twice per week due to low hydrocarbon concentration variability. Water analyses verified the aqueous gasoline removal rate and the UV/oxidation destruction efficiency. It also allowed estimation of the total gasoline discharge to the sanitary sewer, which amounted to less than $5 \mathrm{lb}$ of gasoline in 11 million gal of treated water discharged in 1993.

Because the vapor system accounted for most of the gasoline removal and because it varied much more than the water stream, we sampled it daily, occasionally twice per day. Whenever a significant operational change was made, such as extracting from a different location, we analyzed the vapor stream before and soon after implementing the change. Vapor analyses allowed estimation of vapor-phase gasoline removal and verified air discharge permit compliance.

Sampling Equipment. Specialized sampling equipment was used for drawing samples under vacuum from the vapor system into plastic "tedlar" bags. Sampling bags were inserted into a desiccator equipped with plumbing from the sample bag to the sample port. By drawing air from the desiccator, a vacuum around the sample bag was created producing a pressure gradient from the pipes into the bag. Sample bags were equilibrated with atmospheric temperature and pressure after removing them from the desiccator. This automatic adjustment to ambient conditions allowed laboratory chemists to directly report chemical concentrations from GC data. 
Sampling Error. We discovered several vapor sampling errors during the latter stages of the ARV project, resulting from a poor hose connection between the vapor sampling bag and the vapor piping. These errors occurred mostly when we extracted from any one "steam injection well" used originally for injecting steam around the gasoline spill perimeter. We extracted vapor from these wells to test hydrocarbon concentration variations vs extraction duration and for air sparging testing. Such extraction required greater applied vacuum. To collect a sample from the higher-vacuum piping, the leak potential increased. Ambient air occasionally leaked into sampling bags when poor sampling bag connections were made under these higher vacuum sampling instances.

After discovering this, we replaced the sampling apparatus with a more durable unit that passed pressure testing. Additional vapor samples collected for tracer analyses with a different apparatus simultaneously with the inadvertently diluted samples were later analyzed for hydrocarbons to validate the amount of dilution which occurred in early sampling scenarios.

Sampling Protocol and QA/QC. To prevent additional sampling errors subsequent to the initial effort, we produced a formal sampling protocol with written instructions on sampling apparatus operation. We performed regular vacuum testing of the sampling apparatus to ensure leak-tight sample collection. Occasionally, we analyzed duplicate and triplicate samples collected by different personnel only minutes apart to verify representative sampling methods. All of these quality assurance/quality control efforts indicated that we prevented additional sampling errors.

\section{Chemical Analyses}

Standard analytical methods were used for measuring total BTEX and total petroleum hydrocarbons (TPH). We diversified the analyses by using selective detectors and different gas chromatographs to aid in interpreting patterns of contaminant recovery from both the aqueous and vapor streams.

Sampling Ports. Figures 2 and 3 in the main text present simplified schematics of TFF showing sampling ports for the water treatment system and vapor treatment system, respectively. The following list describes each sampling port designation:

\section{Aqueous Ports}

TFF-SEPI

TFF-UVI

TFF-UV05

TFF-E006-AQ

TFF-MEGA-AQ
Oil/water separator (OWS) influent, located after the ambient air heat exchanger

UV/oxidation system influent

UV/oxidation system effluent

Facility effluent to sanitary sewer, located after the air stripping tanks

Aqueous phase obtained from vapor stream OWS (aqueous condensate from flat plate heat exchanger) 


\section{Gasoline Port}

TFF-MEGA-HC Condensed gasoline obtained from vapor stream OWS ("organic" condensate from flat plate heat exchanger)

\section{Yapor Ports}

ICE-IN

ICE-OUT Internal combustion engine influent, located after the flat plate heat exchanger

TFF-CFI

ICE effluent

TFF-CFO

GAC filter influent (air stripping tank off-gas vapor effluent; aqueous stream)

GAC filter effluent (vapor).

Table A4-2 summarizes the ARV project sampling schedule.

Table A4-3 summarizes the number of samples collected and the number of these samples analyzed for each sampling port during the ARV project.

\section{Analytical Methods}

This section describes analytical equipment used for chemical analyses during the ARV project.

\section{Aqueous and Vapor Analyses}

GC Apparatus. Chromatography was performed using a Hewlett Packard 5890 series II gas chromatograph (GC) outfitted with a photoionization detector (PID) and an electrolytic conductivity detector (ELCD). A low dead volume (LDV) injector port with a transfer line attachment from an O.I. Analytic Corporation Model 4560 Liquid Sample Concentrator (\#6 trap, tenax/silica gel/charcoal) was interfaced to the GC. The GC column was a J \& W Scientific $30 \mathrm{~m} \times 0.53 \mathrm{~mm}$ (inner diameter), fused silica DB624, with a film thickness of 3 micrometers. The GC injector port temperature was maintained at $190^{\circ} \mathrm{C}$. The PID and ELCD temperatures were maintained at $220^{\circ}$ and $900^{\circ} \mathrm{C}$, respectively. A PTA-30 W/S autosampler (Dynatech Precision Sampling Corp.) was employed for aqueous sample analysis. The GC oven temperature profile had an initial temperature and duration of $50^{\circ} \mathrm{C}$ for $5 \mathrm{~min}$. The oven then ramped $6^{\circ} \mathrm{C}$ per minute to a final temperature of $110^{\circ} \mathrm{C}$. A postsample analysis bake-out was employed by ramping the oven at $20^{\circ} \mathrm{C} / \mathrm{min}$ to $170^{\circ} \mathrm{C}$ for $3 \mathrm{~min}$. After the $\mathrm{GC}$ analysis, the oven returned to $50^{\circ} \mathrm{C}$ and equilibrated for $1 \mathrm{~min}$ prior to analyzing the next sample. The Liquid Sample Concentrator (purge and trap) was used for aqueous sample analysis and low VOC concentration vapor sample analysis. The purge, desorb, and bake times for liquid samples were 4,3 , and 20 min, respectively. The purge, desorb, and bake times for vapor samples were 8,3 , and $20 \mathrm{~min}$, respectively. The purge, desorb, and bake temperatures for either liquid or vapor samples were $25^{\circ}, 180^{\circ}$, and $190^{\circ} \mathrm{C}$, respectively. HP Chemstation, an automated GC systems control and data acquisition programmable workstation, was used to gather, process, and archive the GC data. 
Table A4-2. Sampling schedule.

\begin{tabular}{|c|c|c|c|c|c|c|}
\hline \multicolumn{7}{|c|}{ System performance samples } \\
\hline & Mon. & Tues. & Wed. & Thur. & Fri. & Analyses \\
\hline \multicolumn{7}{|c|}{ Sample locations (aqueous) } \\
\hline TFF-SEPI & $1 / d$ & & & 1/d & & TPH,601/602-EDB \\
\hline TFF-UVI & $1 / d$ & & & $1 / d$ & & TPH,601/602-EDB \\
\hline TFF-UVO5 & $1 / d$ & & & $1 / d$ & & TPH,601/602-EDB \\
\hline \multicolumn{7}{|c|}{ Sample locations (vapor) } \\
\hline \multirow[t]{2}{*}{ TFF-ICE-IN } & $2 / d$ & 2/d & $2 / d$ & $2 / d$ & $2 / d$ & FID $^{* *}$ \\
\hline & $1 / d \S$ & $1 / d$ & $1 / d$ & $1 / d \S$ & $1 / d$ & TPH \\
\hline
\end{tabular}

\section{Regulatory samples}

Sample locations (aqueous)

TFF-UVO5

TFF-E006-AQ

1/month
1/quarter

Sample locations (vapor)

TFF-CFO

$1 / d$

1/d

1/d

\section{$\mathbf{1} d$}

$1 / d$

FID**

\section{Notes:}

* 1x/quarter-certified analysis (CLS).

** a field measurement.

*** $1 \mathrm{x} /$ month-certified analysis (CLS).

$\S$ Include BTEX.

$\infty$ Sampled during operation of carbon trailer. 
Table A4-3. Sampling summary.

\begin{tabular}{|c|c|c|}
\hline & Number of samples & Number of analyses \\
\hline \multicolumn{3}{|c|}{ Aqueous sampling port } \\
\hline MEGA & 13 & 26 \\
\hline SEPI & 19 & 38 \\
\hline UVI & 19 & 38 \\
\hline UV05 & 19 & 38 \\
\hline E006 & 2 & 3 \\
\hline Total & 72 & 143 \\
\hline \multicolumn{3}{|c|}{ Vapor sampling port } \\
\hline ICE-IN & 84 & 128 \\
\hline CFO & 1 & 2 \\
\hline ICE-OUT & 1 & 2 \\
\hline Total & 86 & 132 \\
\hline \multicolumn{3}{|l|}{ Wells } \\
\hline GIW-815 & 6 & 11 \\
\hline GIW-820 & 2 & 4 \\
\hline GIW-0820-Lower 7 & 7 & 8 \\
\hline GIW-820-Upper & 12 & 16 \\
\hline Total & 27 & 39 \\
\hline
\end{tabular}


The compounds of interest in our aqueous analyses were BTEX, 1,2-dichloroethane, chloroform, carbon tetrachloride, trichloroethylene, and ethylene dibromide. BTEX and trichloroethylene were quantified by the PID. 1,2-dichloroethane, chloroform, carbon tetrachloride, and ethylene dibromide were quantified by the ELCD.

Aqueous samples were received for 601/602-EDB analysis from TFF-SEPI, TFF-UVI, TFFUV05, and TFF-E006-AQ. The PTA-30 W/S autosampler was used to dilute (if necessary) and transfer the sample to the liquid sample concentrator. The dilutions performed by the autosampler were 1:5, 1:10, and 1:20. The final sample volume was $5 \mathrm{~mL}$. Unpreserved aqueous samples were received in duplicate. One sample was analyzed immediately. The other sample was held for later analysis (confirmation purposes), or until its 2-week expiration date. Expired samples were returned to the sender for disposal.

Chlorobenzene was used as a surrogate to evaluate aqueous VOC recovery. Dicloroproplyene was used as an internal standard to measure GC performance, and to generate the HP Chemstation internal standard sample concentration report.

Vapor samples were received in either 1-L tedlar bags or in $500-\mathrm{mL}$ stainless steel spheres (SSS). Vapor samples were either introduced into the GC by direct injection into the LDV injector port (high VOC concentration samples), or through injection into the purge and trap (low VOC concentration samples). The sample was deemed as a low VOC concentration sample if the concentration was less than 50 ppmv. If the sample concentration was greater than $50 \mathrm{ppmv}$, it was deemed as a high VOC concentration sample. Duplicate injections were made on each sample. The injection volumes for low and high VOC concentration vapor samples were $20 \mathrm{~mL}$ and 10 microliters $(\mu \mathrm{L})$, respectively. Injections were made using either a $25-\mu \mathrm{L}$ or a $50-\mathrm{mL}$ gastight syringe (Hamilton). The $25-\mu \mathrm{L}$ syringe was equipped with a side port needle, and the $50 \mathrm{~mL}$ syringe was equipped with a Teflon stopcock (Supleco).

Low VOC concentration samples were introduced into the GC by injecting a $20-\mathrm{mL}$ sample into the sparge tube (purge and trap) using a 50-mL Hamilton gas-tight syringe. The syringe was fitted with a stopcock and 18 gauge, 1.5-in, needle in order to remove the sample from the tedlar bag sample port. The syringe was flushed with the sample three times to ensure that a homogeneous vapor sample was entrapped within the syringe. Once a sample was drawn into the syringe, the stopcock at the syringe tip was placed in the off position. The needle was removed and the syringe was then attached to the purge and trap sparge tube. The syringe stopcock and the purge and trap sample port valve were then opened, and the sample was injected into the sparge tube. Once the sample was placed in the sparge tube, the purge and trap sample port valve was closed and the purge cycle started manually.

High VOC concentration samples were injected directly onto the GC with a $10-\mu \mathrm{L}$ sample through the LDV injector port using a 25 - or $50-\mu \mathrm{L}$ Hamilton syringe equipped with a side port needle. The syringe needle was inserted into the tedlar bag sample port, and was flushed with sample at least three times to ensure that a homogeneous vapor sample was entrapped within the syringe. Ten microliters were drawn slowly into the syringe, and then the direct GC injection was made. The GC was started manually after sample injection. 


\section{Standards and Reagents}

Neat compounds purchased from Chem Service, Inc., were used to prepare calibrations for aqueous and high VOC concentration vapor samples. Scotty's II certified vapor standards $(1,10$, and $50 \mathrm{ppmv}$ ) were utilized to prepare calibrations for low VOC concentration vapor samples.

Aqueous. A 100-ppm (100 mg/L) working stock solution was prepared in $100 \mathrm{~mL}$ of high purity methanol (B \& J Brand, Baxter Scientific Products). The working stock solution contained all compounds of interest. Working stock solutions were prepared in $120-\mathrm{mL}$ clear glass serum bottles. The serum bottles were capped with Teflon-lined silicone septa and aluminum crimp caps. The septa and caps were replaced after use. GC calibration standards were prepared in $40-\mathrm{mL}$ volatile organic analysis vials (VOAs) (I-Chem) by adding the appropriate volume of the neat reagent to $40 \mathrm{~mL}$ of ultrapure $(0.22$ micrometer filtered) water. The ultrapure water was acquired from a NANOpure ultrapure water system (Barnstead/ Thermolyne), and purged with helium for $30 \mathrm{~min}$ before use. PID GC calibration standards from 2.5 to $2,000 \mathrm{ppb}$ were prepared from a $100-\mathrm{ppm}$ working stock solution. ELCD GC calibration standards from 2.5 to $100 \mathrm{ppb}$ were prepared from a $100-\mathrm{ppm}$ working stock solution. Calibration standards were analyzed and entered into the Chemstation for each dilution factor used. The GC was calibrated every 2 weeks, and GC calibrations were examined daily using calibration checks. Recalibration of the GC would occur any time the calibration check varied $10 \%$ from the anticipated value.

Vapor. When the sample was received, the VOC concentration range was determined by checking previous results or by GC screening. The sample designation and analytical method were then entered into the HP Chemstation. Standards were prepared in a 500-mL stainless steel sphere or 1-L tedlar bags. Neat compounds purchased from Chem Service, Inc., for uncertified standards were prepared to a desired concentration. Standards were made in the laboratory by adding the proper amount of clean air to the tedlar bag and injecting the appropriate quantity of neat VOC into the bag using a Hamilton syringe. The bag was then allowed to stabilize for 20 min prior to use. When the commercial standards were employed, a tedlar bag was filled with the vapor standard and analyzed by GC. Vapor standards were made daily and not reused beyond $24 \mathrm{~h}$. Standards were analyzed weekly, and a response factor (RF) was used to calibrate the GC and quantify the vapor sample concentrations. The RF was checked during the week, and the GC was recalibrated if necessary.

\section{TPH Analyses}

Analyses of total petroleum hydrocarbons (TPH) were performed using an autosampler (PTA-30W/S, Dynatech Precision Sampling Corp.) and a purge and trap concentrator (Model 4460A, Trap \#6: Tenax/Silica Gel/Charcoal, O.I. Corp.) coupled to a Hewlett Packard HP 5890 Series II GC equipped with a flame ionization detector (FID). A fused-silica column (30 $\mathrm{m} \mathrm{x}$ $0.53 \mathrm{~mm}$ i.d.) coated with $1.5 \mu \mathrm{m}$ dimethylpolysiloxane (DB-1, CAT\# 125-1032, J\&W Scientific) was employed. The injector and detector temperatures were $200^{\circ}$ and $220^{\circ} \mathrm{C}$, respectively. The GC oven was held at an initial temperature of $35^{\circ} \mathrm{C}$ for 4 min followed by temperature programming to $80^{\circ} \mathrm{C}$ at $8 \mathrm{deg} / \mathrm{min}$, then to $220^{\circ} \mathrm{C}$ at $12 \mathrm{deg} / \mathrm{min}$, and then to $240^{\circ} \mathrm{C}$ at $20 \mathrm{deg} / \mathrm{min}$ with a final hold at $240^{\circ} \mathrm{C}$ for $2 \mathrm{~min}$. The purge, desorb, and bake times were 11,3 , 
and 20 min, respectively. The desorb and bake temperatures were $180^{\circ} \mathrm{C}$. An HP 3365 Series II Chemstation (DOS) was used for data collection, storage, and integration.

Aqueous. Samples were injected into the purge and trap sparge tube via the autosampler in 5-mL aliquots. For samples of high concentration (i.e., TFF-MEGA-AQ, TFF-SEPI, and TFFUVI), the autosampler was programmed to dilute the sample by a factor of 10 ( $4.5 \mathrm{~mL}$ water added to a 0.5 -mL sample).

Vapor. Samples were injected directly into the $\mathrm{GC}$ in $100-\mu \mathrm{L}$ aliquots via a $100-\mu \mathrm{L}$ gas-tight syringe equipped with a side-port needle (Hamilton). For samples of low hydrocarbon concentration (i.e., TFF-ICE-OUT and TFF-CFO), the sample was injected into the purge and trap sparge tube via a $10-\mathrm{mL}$ glass syringe. Two injections were made from each sample to ensure proper sample handling and representativeness.

Standards and Reagents. The sample of gasoline employed to calibrate the method was free product (weathered gasoline) obtained from well GSW-15 in June 1990 at LLNL.

Aqueous. A working stock solution $(10,000 \mathrm{mg} / \mathrm{L})$ was prepared in $100 \mathrm{~mL}$ of methanol (high purity, B \& J Brand, Baxter Scientific Products) by adding $1.36 \mathrm{~mL}$ weathered gasoline (density $=0.735 \mathrm{~g} / \mathrm{mL}$ ). Standards were prepared from this working stock in the range of 250 to $25,000 \mathrm{ppm}$ by adding 1 to $100 \mu \mathrm{L}$ of this working stock to clean water (filtered by a NANOpure ultrapure water system, Model \#D4741, Barnstead/Thermolyne; followed by a 30-min sparge with Helium) in 40-mL VOAs, (I-Chem). Appropriate standard concentrations and dilution factors (one to ten) were chosen that ranged between the concentrations of a given sample. Calibrations were run as required (generally monthly). Calibration checks were performed in triplicate prior to analysis of each set of samples, and the calibrations were deemed sufficient if each of the three check standards was $\pm 10 \%$ of its expected value.

Vapor. Standards were prepared in $250-\mathrm{mL}$ glass vessels. The glass vessels were equipped with two stopcocks and a septum port. A new septa was placed on the vessel, and the vessel was evacuated on the house vacuum for $30 \mathrm{~min}$. The vessel was then removed from the house vacuum, and 1 to $10 \mu \mathrm{L}$ of gasoline was injected into the vessel (resulting concentration: 832 to $8,320 \mathrm{ppmv}$ ). The vacuum was then relieved by a rapid turning of the stopcocks, and allowed to equilibrate for $30 \mathrm{~min}$ before analysis. Three standards of differing concentrations were prepared, and each one was injected twice (in 100- $\mu \mathrm{L}$ aliquots) to ensure proper sample handling.

TPH Window. For this experiment, TPH was defined as the concentration of all compounds that elute within the $\mathrm{C}_{6}$ to $\mathrm{C}_{12}$ window (hexane to dodecane).

Vapor. TPH was reported as mass per unit volume $(\mathrm{mg} / \mathrm{L}$ or $\mathrm{ng} / \mathrm{\mu L}$ ) and parts per million by volume (ppmv). Reporting ppmv requires a molecular weight to convert mass/volume to ppmv (mol/mol). We report ppmv based on the molecular weight of hexane, $86 \mathrm{~g} / \mathrm{mol}$. The calculation is as follows: 


$$
\begin{gathered}
\text { ppmv }=\frac{\text { Mass of gas/molecular wt. of gas }}{\text { Volume of air/molecular wt. of air }} \cdot 10^{6} \\
\mathrm{ppmv}=\frac{\text { Volume }(\mu \mathrm{L}) \times \text { density }(\mathrm{g} / \mathrm{mL}) \times 1 \mathrm{~mL} / 1000 \mu \mathrm{L} \times 24.4 \mathrm{~L} / \mathrm{mol}}{0.25 \mathrm{~L} \times \mathrm{MW}(\mathrm{g} / \mathrm{mol})} \cdot 10^{6} \\
\mathrm{ppmv}=\frac{4 \times 24.4 \times \text { density }(\mathrm{g} / \mathrm{mL}) \times \text { volume }(\mu \mathrm{L})}{1000 \times 10^{6}} \cdot 1 \mathrm{gW}(\mathrm{mol})
\end{gathered}
$$

In the early part of the second phase of the DUSDP conducted at TFF (May through July 1993), some samples were observed to contain a large portion of low molecular weight compounds that elute prior to hexane and, therefore, outside of the TPH window. For these samples, we decided that the TPH value gave an inaccurate picture of the total amount of organic compounds in a given sample. For this reason, we defined a new value, which we termed "total hydrocarbons." The window for total hydrocarbons can be described as $C_{1}$ to $C_{12}$ (methane to dodecane), because we included all compounds that elute prior to dodecane in this calculation.

Field measurements were made using a Foxboro organic vapor analyzer (OVA) equipped with an FID. These measurements provided approximate hydrocarbon concentrations in discharged air for regulatory compliance.

\section{Quality Assurance/Quality Control (QA/QC)}

\section{Water Analysis QA/QC}

\section{Surrogate Recoveries}

Chlorobenzene was used as the surrogate spiking compound, and was added to every aqueous sample, including blanks. Project methods called for QC limits ranging between 80 and $120 \%$ of recovered surrogate, in compliance with EPA recommendations. Table A4-7 shows recoveries were always between the $\mathrm{QC}$ limits $(E P A, 1988)$.

\section{Field Spikes (Percent Recovery)}

For analytical QA/QC, matrix spikes and matrix spike duplicates were performed throughout the duration of the experiment. The spiking levels were twice the estimated amount for designated compounds present in the sample. The spiking compounds were added to the matrix spike and matrix spike duplicate aliquots of the sample before being placed on the purge-and-trap apparatus.

\section{Internal Standard}

The Internal Standard Method (ISTD) using the HP3365 Series II Chemstation Software calculates each peak separately and reports the absolute amount of material for each calibrated analyte. The results are independent of sample size, giving the most accurate analysis scheme for liquid samples. The internal standard, cis-1,3-dichloropropene, was added in a known 
amount to every sample. Because this internal standard was present in both unknown and calibrated samples, its presence was detected in all analyses and served as a reference or normalizing factor.

\section{Internal Standard Calculation}

Actual amount of $\mathrm{y}=$ Amount ratio $\mathrm{x}$ Actual amount of ISTD $\mathrm{x} M$ where:

$$
\text { Amount ratio }=\frac{(\text { Area }) y}{(\text { Area }) \text { ISTD }} \times \frac{\text { (Response)y }}{(\text { Response)ISTD }}
$$

- $\quad M$ is the multiplier specified on the calibration setting panel.

- (Area) $y$ is the area of peak $y$.

- (Area) ISTD is the area of the internal standard peak.

- (Response) y is the amount per unit area of peak $y$.

- (Response) ISTD is the amount per unit area of the internal standard peak.

\section{Detection Limits}

Limits of detection (LOD) were set using the American Chemical Society recommendation that detection levels be set at three times the standard deviation of the noise level of the analytic measurement and that quantification levels be set at ten times the standard deviation. The range between three and ten times the standard deviation is considered uncertain for the purposes of quantification. GC limits of detection are primarily governed by the detector employed, detector sensitivity to the compound of interest, and sample volume. The LODs can be found in Table A4-4, which presents detection limits for various VOCs by detector type (Analytic Chemistry, 1983).

Table A4-4. Detection limits.

\begin{tabular}{|l|c|c|c|}
\hline \multicolumn{1}{|c|}{ vOC } & $\begin{array}{c}\text { Photoionization } \\
\text { detector (PID) } \\
(\mu \mathrm{g} / \mathrm{L})\end{array}$ & $\begin{array}{c}\text { Electrolytic conductivity } \\
\text { detector (ELCD) } \\
(\mu \mathrm{g} / \mathrm{L})\end{array}$ & $\begin{array}{c}\text { Flame ionization } \\
\text { detector (FID) } \\
(\mu \mathrm{g} / \mathrm{L})\end{array}$ \\
\hline Benzene & 0.2 & & \\
\hline Toluene & 0.2 & & \\
\hline Ethyl benzene & 0.2 & & \\
\hline p,m-xylene & 0.2 & 0.2 & \\
\hline o-xylene & 0.2 & 0.2 & \\
\hline 1,2-dichloroethane & 0.2 & 0.2 & \\
\hline Trichloroethene & & 0.2 & \\
\hline Ethylene dibromide & 0.2 & & \\
\hline Chlorobenzene & & & \\
\hline TPH & 0 & & \\
\hline \hline
\end{tabular}




\section{Criteria for Recalibration}

Calibration checks were run daily using $25,100,2,500 \mu \mathrm{g} / \mathrm{L}$ of a list stock solution, which contained BTEX, 1,2-dichloroethane, trichloroethene, and ethylene dibromide. If the calibration check standards varied by $10 \%$ of the anticipated value, the instrument was recalibrated. All methods were calibrated by external calibration procedures using five to seven analyte concentrations. A National Institute of Standards and Technology (NIST) traceable external check sample was analyzed when a new calibration was performed.

\section{Accuracy and Precision}

Data quality criteria established in terms of precision and accuracy are presented in Table A4-5. Precision objectives are expressed in terms of relative percent difference (RPD). RPD is defined as the difference between two values, divided by their average. Precision was determined through the use of laboratory duplicates. The analyses of the duplicates met the analytical precision objectives of the project, which were $\pm 20 \%$.

Accuracy objectives were evaluated through the use of laboratory control samples and matrix spike/matrix spike duplicate analyses. Laboratory control samples are clean reference samples spiked with a known concentration of target analytes.

Table A4-5. Data quality criteria and objectives.

\begin{tabular}{ccc}
\hline Parameter & $\begin{array}{c}\text { Precision } \\
\text { (RPD) }\end{array}$ & $\begin{array}{c}\text { Accuracy } \\
\text { (matrix \% recovery) }\end{array}$ \\
\hline TPH & 3 & $80-120$ \\
Benzene & 2 & $80-120$ \\
Toluene & 2 & $80-120$ \\
Ethyl benzene & 2 & $80-120$ \\
?-, m-Xylene & 2 & $80-120$ \\
o-Xylene & 2 & $80-120$ \\
\hline \hline
\end{tabular}

\section{Blank Analyses}

Method blanks were analyzed for every three to four unknown samples showing no contaminants greater than the detection limit for the method being used.

\section{Vapor Analysis QA/QC}

\section{Blank Spheres}

Prior to use, 500-mL stainless steel spheres (SSSs) were cleaned, pressure checked, and analyzed for contaminants. Contaminated SSSs were flushed with clean air for 45 min to remove residual contamination. After the flushing, the valves were closed. GC analysis was then 
performed using an equal or larger sample than required by the method being performed. FID and PID/ELCD detectors were used to check for residual contamination.

\section{Precision}

The data quality criteria are established in terms of precision (see Table A4-6). Vapor samples were not spiked; therefore, accuracy was not calculated.

Precision objectives are presented in Table A4-6. From duplicate analyses, the average difference, or average range, is calculated by summing all the differences (absolute values) and dividing by the number of observations: $R=\Sigma \mathrm{d}_{\mathrm{j}} / \mathrm{n}$. This is converted to standard deviation(s) by dividing by 1.128 .

Table A4-6. Data Quality Criteria and Objectives

\begin{tabular}{cc}
\hline & $\begin{array}{c}\text { Precision } \\
\text { (s) }\end{array}$ \\
\hline Parameter & 2.3 \\
TPH & 2.7 \\
\hline
\end{tabular}

\section{Criteria for Recalibration}

All analytical methods used were calibrated by external calibration procedures, using two to three standard concentrations, depending upon the method. A new calibration was performed at least once per quarter or as needed when the response factors varied by $10 \%$ of the anticipated value. Calibration checks were run daily using 10 and $50 \mathrm{mg} / \mathrm{L}$ BTEX external standard purchased from Scotty Specialty Gas, Inc.

\section{Ground Water Analytical Results}

This section describes BTEX and TPH trends for various ground water treatment system sampling reports.

SEPI is the designation for the untreated ground water sampling port located after the ambient air heat exchanger (Fig. 2, main text). Early in the ARV project, TPH concentrations in extracted ground water were around $7 \mathrm{ppm}$. TPH concentrations increased to $20 \mathrm{ppm}$ by the third day of continuous operation and then decreased to between 5 and $10 \mathrm{ppm}$ for the remainder of continuous operations (Fig. A4-1).

Total BTEX concentrations in extracted ground water showed a similar trend to TPH concentrations, starting around $3 \mathrm{ppm}$ and increasing to about $8 \mathrm{ppm}$ by the third day (Fig. A4-1). Concentrations averaged about $3 \mathrm{ppm}$ for the remaining 67 days of continuous ground water pumping. Total BTEX constituted about $35 \%$ of TPH except for an increase to about $75 \%$ on day 38. A 2.8-fold increase in total BTEX concentration was observed between days 36 and 


\section{HdL fo \% XGLG}

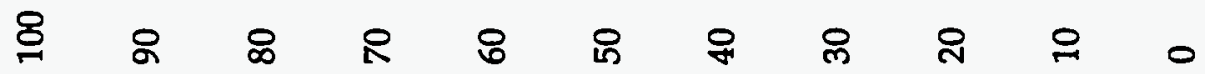
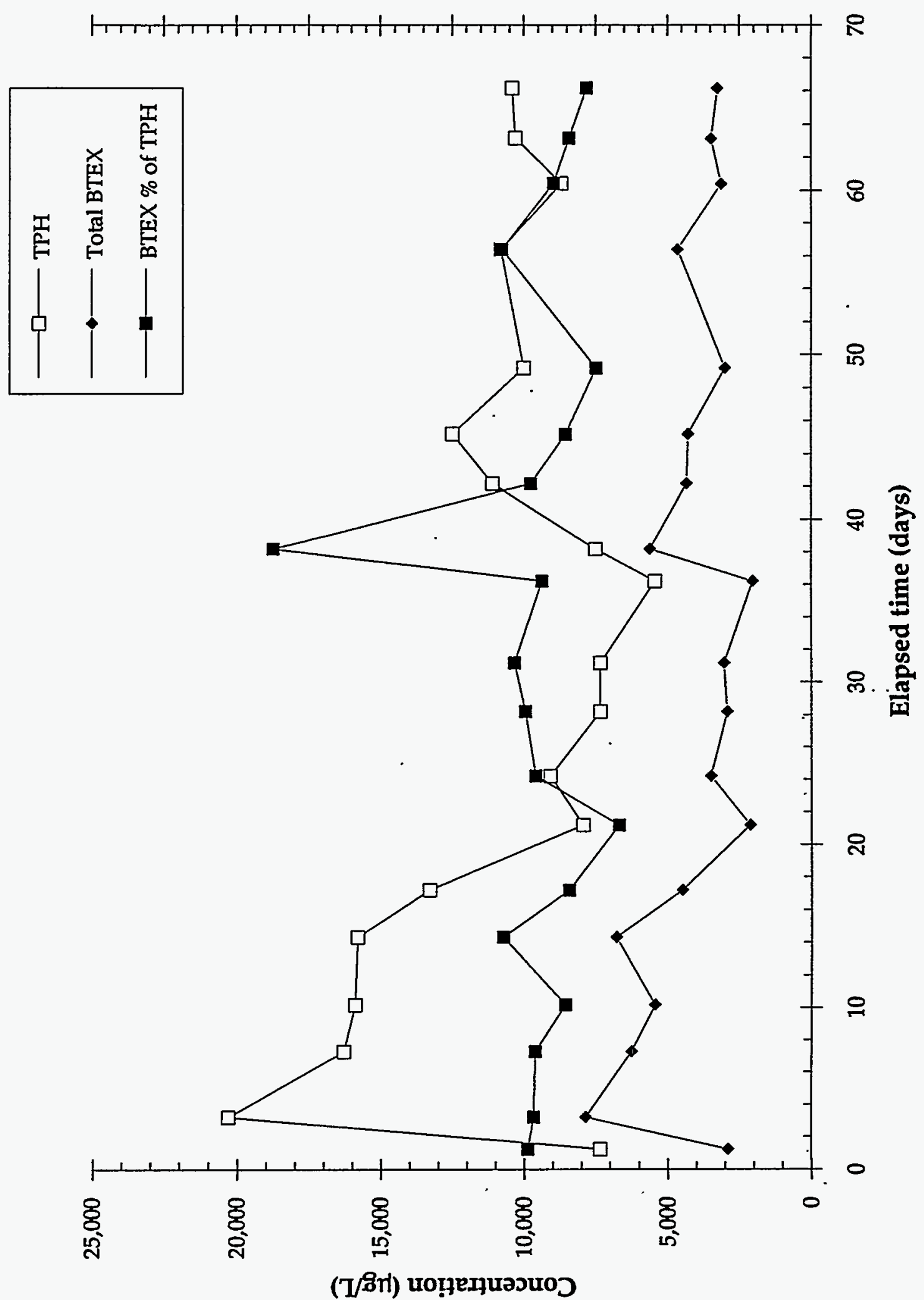

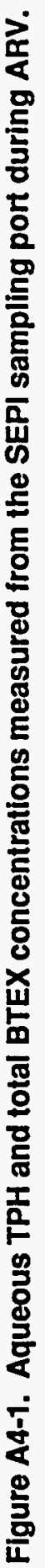


38, while only a 1.4-fold increase in TPH was observed. The data point may be an outlier except that the original chromatogram for BETX analysis shows a large increase in benzene.

The highest percent of the BTEX components in the aqueous stream consisted of toluene averaging about $36 \%$ with maximum concentrations of $2.9 \mathrm{ppm}$ (Fig. A4-2). Total xylenes were also high and averaged $46 \%$ of total BTEX with a high of about $3.2 \mathrm{ppm}$. Benzene averaged $12 \%$ of total BTEX, ranging between 0.2 and $1 \mathrm{ppm}$ during the $70-\mathrm{d}$ period. Ethyl benzene constituted 7\% of total BTEX with concentrations between 0.1 and $0.6 \mathrm{ppm}$ during the same period.

The TCE concentration in extracted ground water averaged about $21 \mathrm{ppb}$. We detected no 1,2-DCA or EDB during the ARV continuous operations (Fig. A4-3).

UVI is the designation of the aqueous sampling port before entering the $U \mathrm{UV}^{-} \mathrm{H}_{2} \mathrm{O}_{2}$ oxidation system and downstream of SEPI (Fig. 2, main text). Concentrations of TPH and total BTEX at UVI followed the same pattern as those at the SEPI port, but were usually lower by $20 \%$ or less (Fig. A4-4). Percentages of the BTEX components were similar to percentages measured at SEPI except ethyl benzene was as low as $3 \%$ and xylenes as high as about $54 \%$ of the total.

TCE concentrations in UVI were typically $24 \%$ lower than from SEPI. EDB and 1,2-DCA were not detected in UVI samples above $4.0 \mathrm{ppb}$.

UV05 is the designation of the aqueous sampling port located downstream of the $\mathrm{UV} / \mathrm{H}_{2} \mathrm{O}_{2}$ oxidation system (Fig. 1, main text). UV/oxidation destruction efficiencies were calculated using BTEX concentrations measured from the UVI and UV05 sampling ports. BTEX destruction efficiencies greater than $90 \%$ were achieved during the first 24 days of continuous pumping (Fig. A4-5). Destruction efficiencies then decreased from $94 \%$ to about $26 \%$ during the following 46 days. Total BTEX concentrations in the UV/oxidation effluent varied between 0.03 and 3 ppm.

UV/oxidation destroyed $50 \%$ of the extracted TCE (Table A4-7).

Treated ground water was monitored at the E006-AQ port (Fig. 2, main text) before being discharged to the sanitary sewer after air stripping. BTEX, 1,2-DCA, and TCE were not detected in the samples collected from this sampling port.

MEGA-AQ is the designation for the condensed and separated water sampling port located downstream of the small oil-water separator (Fig. 3, main text). We began sampling the aqueous effluent 14 days after continuous operations began. The TPH concentration in separated water was about $65 \mathrm{ppm}$, decreasing to about $43 \mathrm{ppm}$ after 21 days, and remaining fairly constant at $42 \mathrm{ppm}$ for the remaining 49 days (Fig. A4-6). Total BTEX concentrations remained constant around $10 \mathrm{ppm}$ throughout the 70-day project (Fig. A4-7). The composition of BTEX compounds in separated gasoline was typically about $5 \%$ benzene, $8 \%$ ethyl benzene, $28 \%$ toluene, and $59 \%$ total xylenes.

\section{Vapor Treatment System Analytical Results}

Sample concentrations changed over time as the facility was extracting VOCs. Generally, samples collected from ICE-OUT and CFO contained low VOC concentrations, and samples 

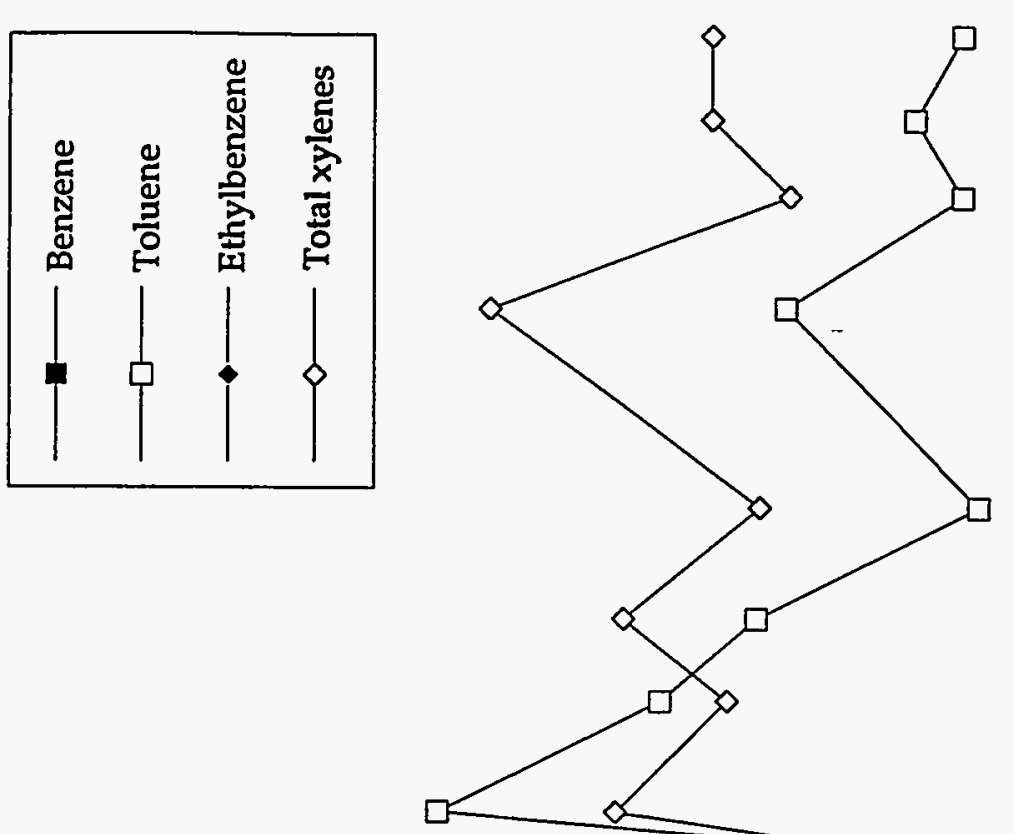

R

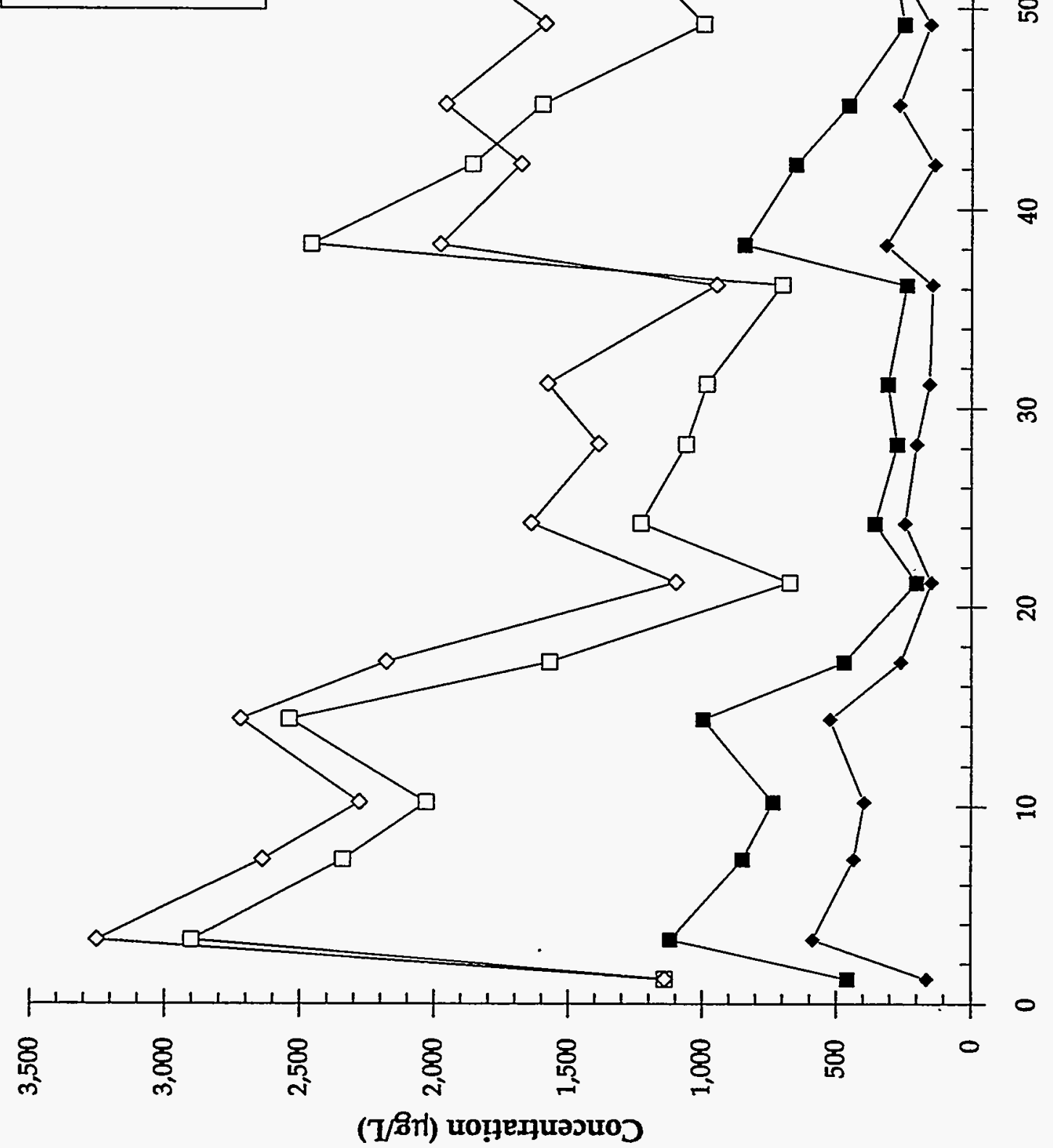




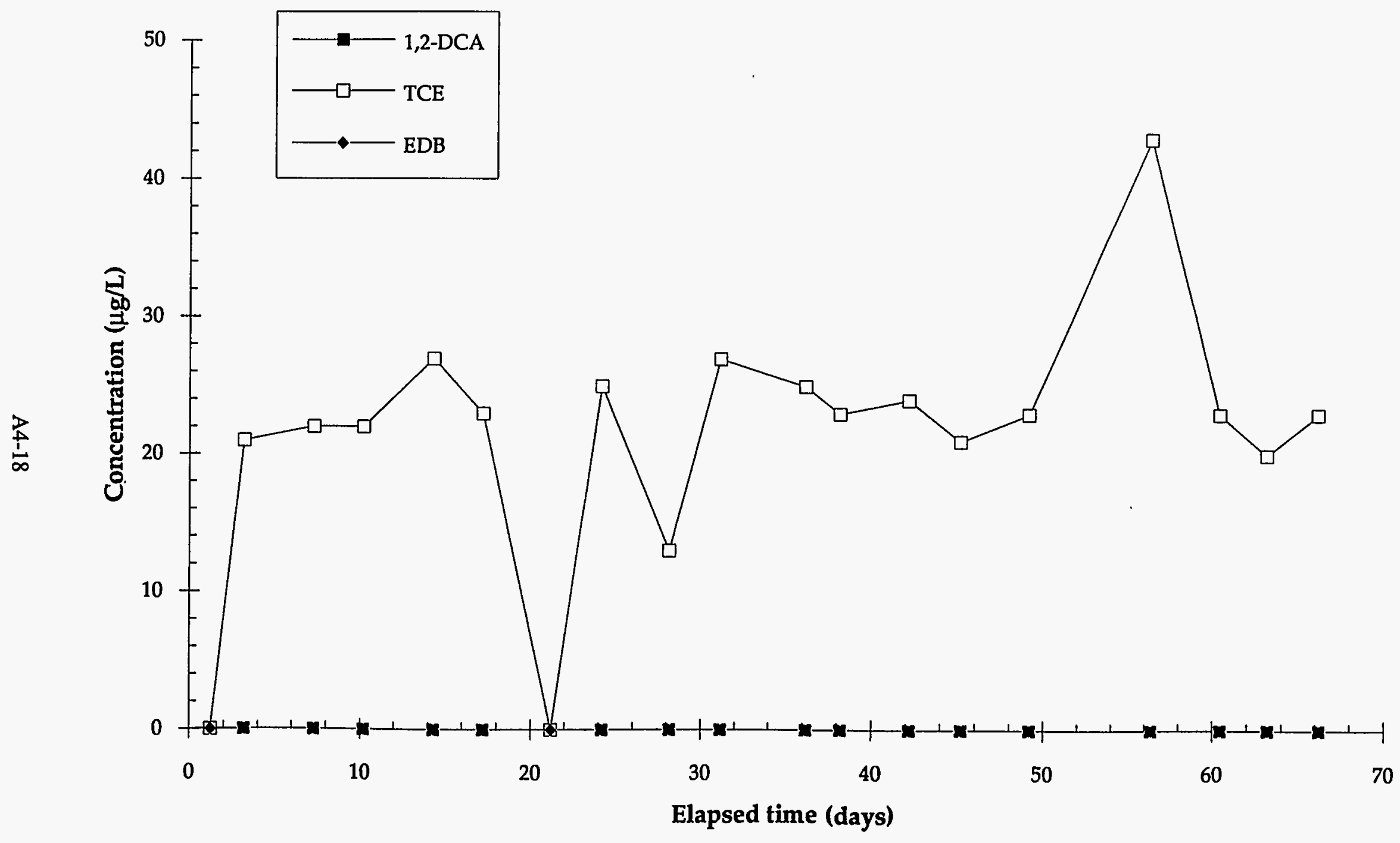

Figure A4-3. 1,2-DCA, TCE and EDB aqueous concentrations measured from the SEPI sampling port during ARV. 


\section{HdL fo \% XGLG}

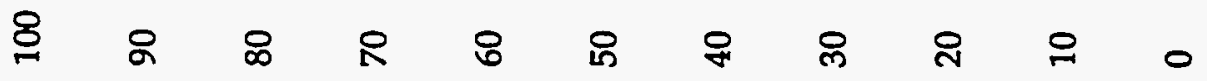
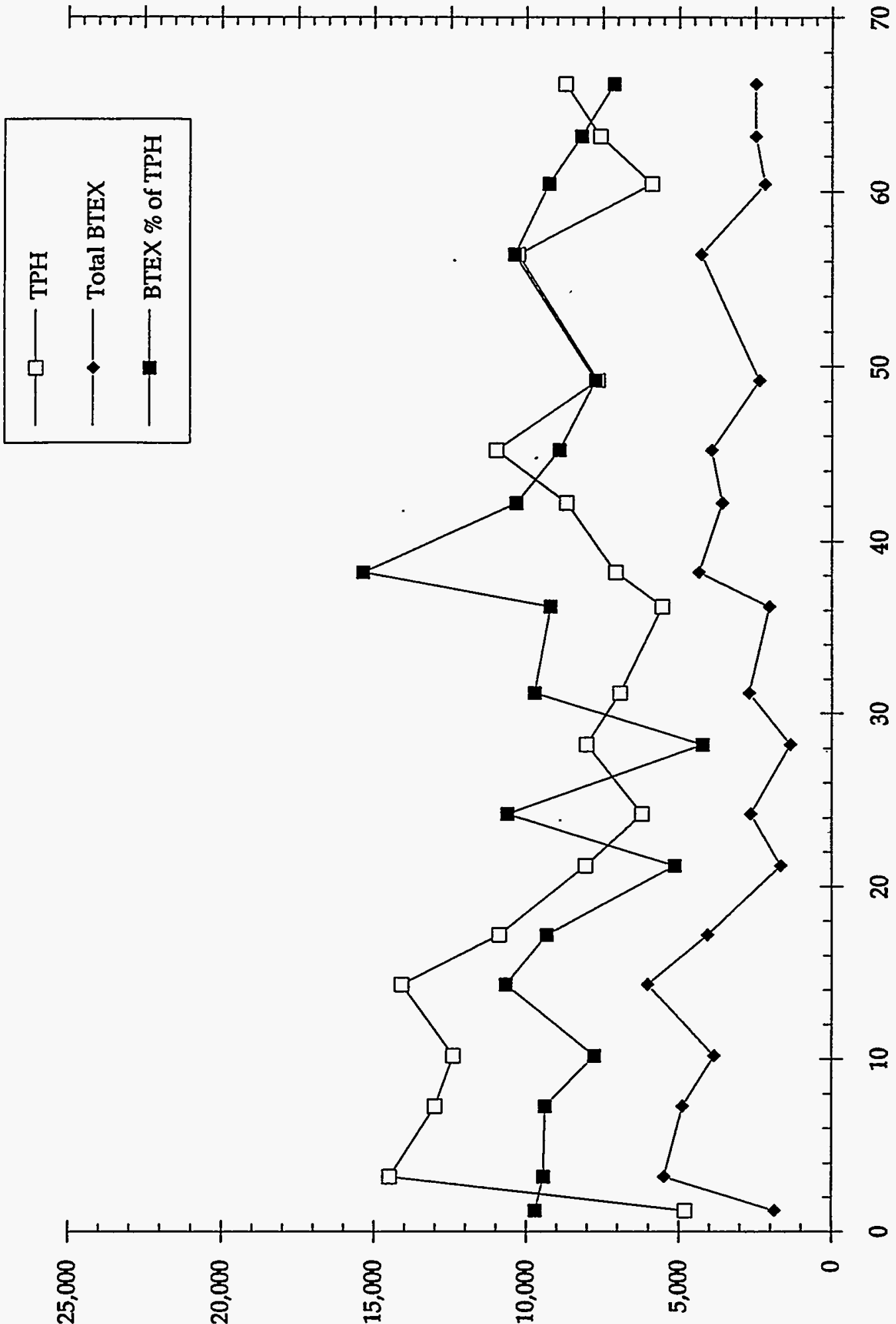

R

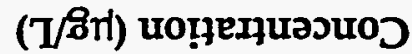




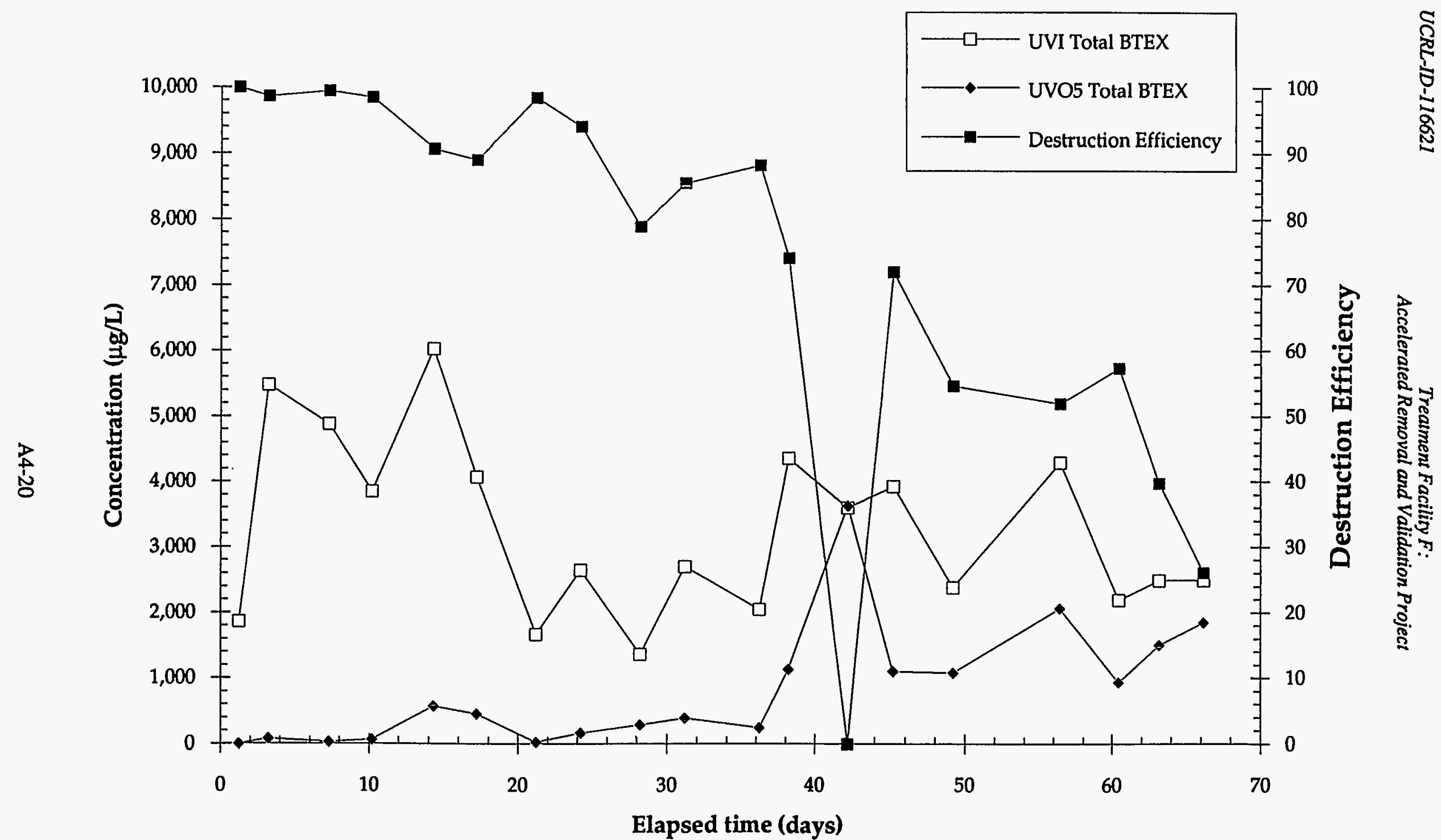

Figure A4-5. Aqueous-phase BTEX concentrations measured from TFF-UV1/TFF-UV05. 
Table A4-7. ARV project aqueous analytic results.

\begin{tabular}{|c|c|c|c|c|c|c|c|c|c|c|c|c|c|}
\hline Date sampled & $\begin{array}{l}\text { Elapsed time } \\
\text { (days) }\end{array}$ & $\begin{array}{l}\text { Total BTEX } \\
(\mu g / L)\end{array}$ & $\begin{array}{c}\text { BTEX } \\
\text { (\% of TPH) }\end{array}$ & $\begin{array}{c}\text { Benzene } \\
(\mu g / L)\end{array}$ & $\begin{array}{c}\text { Toluene } \\
(\mu g / L)\end{array}$ & $\begin{array}{c}\text { Ethylbenzene } \\
(\mu \mathrm{g} / \mathrm{L})\end{array}$ & $\begin{array}{c}\text { Total xylenes } \\
(\mu g / L)\end{array}$ & $\begin{array}{l}\text { TPH* } \\
(\mu g / L)\end{array}$ & $\begin{array}{c}1,2-D C A \\
(\mu g / L)\end{array}$ & $\begin{array}{c}\text { TCE } \\
(\mu g / L)\end{array}$ & $\begin{array}{c}\text { EDB } \\
(\mu g / L)\end{array}$ & $\begin{array}{l}\text { Surrogate"* } \\
\text { (\% recovery) }\end{array}$ & \\
\hline \multicolumn{14}{|l|}{ TFF-MEGA-AQ } \\
\hline $10 / 18 / 93$ & 14.38 & 12,500 & 19.3 & 586.0 & 3,530 & 993.0 & $7, \mathbf{4 2 0}$ & 64,800 & ND & ND & ND & 80 & \\
\hline $10 / 21 / 93$ & 1721 & 12,600 & 25.1 & 609.0 & 3,480 & 1030.0 & 7,490 & 50,100 & ND & ND & ND & 94 & \\
\hline $10 / 25 / 93$ & 21.21 & 10,600 & 23.2 & 485.6 & 2,910 & 856.2 & 6,320 & 45,700 & ND & ND & ND & 91 & \\
\hline $10 / 28 / 93$ & 24.21 & 12,000 & 28.0 & 578.0 & 3,400 & 1020.0 & 6,970 & 42,800 & ND & ND & ND & 110 & \\
\hline $11 / 01 / 93$ & 28.21 & 11,400 & 26.9 & 494.0 & 3,140 & 938.0 & 6,780 & 42,400 & ND & ND & ND & 95 & \\
\hline $11 / 04 / 93$ & 31.21 & 11,900 & 26.7 & 590.0 & 3,400 & 990.0 & 6,870 & 44,500 & ND & ND & ND & 110 & \\
\hline $11 / 09 / 93$ & 36.21 & 9,800 & 22.6 & 480.0 & 2,750 & 835.0 & 5,740 & 43,400 & ND & ND & ND & 91 & \\
\hline $11 / 11 / 93$ & 38.21 & 8,420 & 20.5 & 404.0 & 2,340 & 659.0 & 5,020 & 41,000 & ND & ND & ND & 87 & \\
\hline $11 / 15 / 93$ & 42.21 & 8,640 & 18.9 & $\mathbf{5 4 7 . 0}$ & 2,530 & 680.0 & 4,880 & 45,700 & ND & 2.4 & ND & 76 & \\
\hline $11 / 18 / 93$ & 45.21 & 9,130 & 16.8 & 566.0 & 2,670 & 711.0 & 5,180 & 54,500 & ND & ND & ND & 82 & \\
\hline $11 / 22 / 93$ & 49.21 & 9,810 & 21.1 & 289.0 & 2,760 & 807.0 & 5,950 & 46,600 & ND & ND & ND & 121 & \\
\hline $12 / 03 / 93$ & 60.46 & 9,190 & 20.9 & 350.0 & 2,540 & 735.0 & 5,560 & 43,900 & ND & ND & ND & 85 & $\overrightarrow{2}$ \\
\hline $12 / 06 / 93$ & 63.21 & 7,650 & 18.0 & 251.0 & 2,070 & 629.0 & 4,700 & 42,500 & ND & ND & ND & 93 & 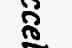 \\
\hline \multicolumn{14}{|l|}{ TFF-SEPI } \\
\hline $10 / 05 / 93$ & 1.25 & 2,910 & 39.5 & 459.0 & 1,140 & 164.0 & 1,140 & 7,360 & ND & ND & ND & - & 2 \\
\hline $10 / 07 / 93$ & 3.21 & 7,860 & 38.7 & 1120.0 & 2,900 & 588.0 & 3,250 & 20,300 & ND & 21.0 & ND & 120 & \\
\hline $10 / 11 / 93$ & 7.29 & 6,270 & 38.5 & 850.0 & 2,340 & 437.0 & 2,640 & 16,300 & ND & 22.0 & ND & 113 & \\
\hline $10 / 14 / 93$ & 10.21 & 5,450 & 34.3 & 737.0 & 2,030 & 398.0 & 2,280 & 15,900 & ND & 22.0 & ND & 90 & \\
\hline 10/18/93 & 14.33 & 6,780 & 42.9 & $\mathbf{9 9 9 . 0}$ & 2,540 & $\mathbf{5 2 4 . 0}$ & 2,720 & 15,800 & ND & 27.0 & ND & 113 & $2=$ \\
\hline $10 / 21 / 93$ & 1721 & 4,480 & 33.7 & 473.0 & 1,570 & 262.0 & 2,180 & 13,300 & ND & 23.0 & ND & 108 & $\mathbf{y}$ \\
\hline $10 / 25 / 93$ & 21.21 & 2,120 & 26.7 & 204.0 & 674 & 149.0 & 1,100 & 7,930 & ND & ND & ND & 110 & \\
\hline $10 / 28 / 93$ & 24.21 & 3,480 & 38.3 & 358.0 & 1,230 & 247.0 & 1,640 & 9,080 & ND & 25.0 & ND & 116 & \\
\hline $11 / 01 / 93$ & 28.21 & 2,920 & 39.8 & 276.0 & 1,060 & 203.0 & 1,390 & 7,340 & ND & 13.0 & ND & 109 & \\
\hline $11 / 04 / 93$ & 31.21 & 3,030 & 41.3 & 309.0 & 985 & 136.0 & 1,580 & 7,330 & ND & 27.0 & ND & 145 & 5 \\
\hline $11 / 09 / 93$ & 36.21 & 2,030 & 37.5 & 240.0 & 703 & 143.0 & 947 & 5,420 & ND & 25.0 & ND & 108 & \\
\hline $11 / 11 / 93$ & 38.21 & 5,610 & 74.9 & 843.0 & 2,460 & 316.0 & 1,980 & 7,490 & ND & 23.0 & ND & 99 & $\overline{7}$ \\
\hline $11 / 15 / 93$ & 42.21 & 4,340 & 39.1 & 654.0 & 1,860 & 137.0 & 1,680 & 11,100 & ND & 24.0 & ND & 86 & $y$ \\
\hline $11 / 18 / 93$ & 45.21 & 4,280 & 34.2 & 456.0 & 1,600 & 269.0 & 1,960 & 12,500 & ND & 21.0 & ND & 91 & \\
\hline $11 / 22 / 93$ & 49.21 & 2,990 & 29.9 & 250.0 & 998 & 154.0 & 1,590 & 10,000 & ND & 23.0 & ND & 86 & กั \\
\hline $11 / 29 / 93$ & 56.42 & 4,650 & 43.1 & 343.0 & 1,520 & 459.0 & 2,320 & 10,800 & ND & 43.0 & ND & 100 & \\
\hline $12 / 03 / 93$ & 60.46 & 3,120 & 35.9 & $\mathbf{3 0 8 . 0}$ & 1,040 & 254.0 & 1,510 & 8,700 & ND & 23.0 & ND & 95 & \\
\hline $12 / 06 / 93$ & 63.21 & 3,470 & 33.7 & 357.0 & 1,170 & 222.0 & 1,720 & 10,300 & ND & 20.0 & ND & 91 & \\
\hline $12 / 09 / 93$ & 66.21 & 3,250 & 31.3 & 292.0 & 1,040 & 197.0 & 1,720 & 10,400 & ND & 23.0 & ND & 98 & \\
\hline \multicolumn{14}{|l|}{ TFF-UVI } \\
\hline $10 / 05 / 93$ & 1.25 & 1,860 & 38.8 & 332.0 & 601 & 10.0 & 920 & 4,790 & ND & ND & ND & - & \\
\hline $10 / 07 / 93$ & 3.21 & 3,480 & 37.8 & 914.0 & 1,710 & ND & 2,860 & 14,500 & ND & 17.0 & ND & 118 & \\
\hline $10 / 11 / 93$ & 7.29 & 4,880 & 37.5 & 748.0 & 1,570 & 25.0 & 2,540 & 13,000 & ND & 16.0 & ND & • & \\
\hline $10 / 14 / 93$ & 10.21 & 3,850 & 31.0 & 573.0 & 1,260 & 40.0 & 1,980 & 12,400 & ND & 18.0 & ND & 89 & \\
\hline $10 / 18 / 93$ & 14.33 & 6,020 & 42.7 & 935.0 & 2,150 & 227.0 & 2,710 & 14,100 & ND & 23.0 & ND & 122 & \\
\hline $10 / 21 / 93$ & 1721 & 4,070 & 37.3 & 406.0 & 1,260 & 126.0 & 2,280 & 10,900 & ND & 19.0 & ND & 113 & \\
\hline
\end{tabular}


Table A4-7 (continued)

\begin{tabular}{|c|c|c|c|c|c|c|c|c|c|c|c|c|c|}
\hline Date sampled & $\begin{array}{r}\text { Elaps } \\
\text { (dd }\end{array}$ & $\begin{array}{l}\text { d time } \\
\text { ys) }\end{array}$ & $\begin{array}{c}\text { Total BTEX } \\
(\mu g / L)\end{array}$ & $\begin{array}{c}\text { BTEX } \\
\text { (\% of TPH) }\end{array}$ & $\begin{array}{c}\text { Benzene } \\
(\mu g / L)\end{array}$ & $\begin{array}{c}\text { Toluene } \\
(\mu g / L)\end{array}$ & $\begin{array}{c}\text { Ethylbenzene } \\
(\mu g / L)\end{array}$ & $\begin{array}{c}\text { Total xylenes } \\
(\mu \mathrm{g} / \mathrm{L})\end{array}$ & $\begin{array}{l}\mathrm{TPH}^{*} \\
(\mu g / \mathrm{L})\end{array}$ & $\begin{array}{c}\text { 1,2-DCA } \\
(\mu \mathrm{g} / \mathrm{L})\end{array}$ & $\begin{array}{c}\text { TCE } \\
(\mu g / L)\end{array}$ & $\begin{array}{c}\text { EDB } \\
(\mu g / L)\end{array}$ & $\begin{array}{l}\text { Surrogate** } \\
\text { (\% recovery) }\end{array}$ \\
\hline \multicolumn{14}{|c|}{ TFF-UVI (continued) } \\
\hline $10 / 28 / 93$ & & 24.21 & 2,640 & 42.5 & 283.0 & 851 & 68.0 & 1,440 & 6,210 & ND & 20.0 & ND & 118 \\
\hline $11 / 01 / 93$ & & 28.21 & 1,360 & 16.9 & 93.0 & 164 & ND & 1,100 & 8,030 & ND & 6.9 & ND & 99 \\
\hline $11 / 04 / 93$ & & 3121 & 2,700 & 39.0 & 269.0 & 917 & 152.0 & 1,360 & 6,930 & ND & 22.0 & ND & 108 \\
\hline $11 / 09 / 93$ & & 36.21 & 2,050 & 36.9 & 245.0 & 711 & 155.0 & 940 & 5,560 & ND & 22.0 & ND & 108 \\
\hline $11 / 11 / 93$ & & 38.21 & 4,360 & 61.5 & 753.0 & 1,790 & $\mathbf{4 1 . 0}$ & 1,780 & 7,090 & ND & 20.0 & ND & 89 \\
\hline $11 / 18 / 93$ & & 45.21 & 3,930 & 35.7 & 409.0 & 1,410 & 234.0 & 1,880 & 11,000 & ND & 20.0 & ND & 72 \\
\hline $11 / 22 / 93$ & & 49.21 & 2,380 & 31.0 & 188.0 & 737 & 31.0 & 1,420 & 7,670 & ND & 19.0 & ND & 88 \\
\hline $11 / 29 / 93$ & & 56.42 & 4,290 & 41.7 & 324.0 & 1,380 & $\mathbf{3 8 7 . 0}$ & 2,200 & 10,300 & ND & 35.0 & ND & 92 \\
\hline $12 / 03 / 93$ & & 60.46 & 2,190 & 37.1 & 215.0 & 534 & 4.5 & 1,430 & 5,900 & ND & 12.0 & ND & 97 \\
\hline $12 / 06 / 93$ & & 63.21 & 2,490 & 32.8 & 286.0 & 803 & 88.0 & 1,310 & 7,580 & ND & 17.0 & ND & 88 \\
\hline $12 / 09 / 93$ & & 66.21 & 2,490 & 28.5 & 206.0 & 626 & 34.0 & 1,630 & 8,730 & ND & 20.0 & ND & 93 \\
\hline \multicolumn{14}{|l|}{ TFF-UVO5 } \\
\hline $10 / 05 / 93$ & & 1.25 & ND & .0 & ND & ND & ND & ND & 57 & ND & ND & ND & • \\
\hline $10 / 07 / 93$ & & 3.21 & 76 & .9 & 11.0 & 25 & 2.0 & 38 & 8,540 & 1.6 & 2.3 & ND & 56 \\
\hline $10 / 14 / 93$ & & 10.21 & 62 & 2.0 & 9.1 & 21 & 4.9 & 27 & 3,040 & 2.0 & 2.4 & ND & 59 \\
\hline $10 / 18 / 93$ & & 14.33 & 569 & 32.7 & 90.0 & 215 & 32.0 & 232 & 1,740 & 2.4 & 12.0 & ND & 73 \\
\hline $10 / 21 / 93$ & & 17.21 & 453 & 32.6 & 47.0 & 152 & 31.0 & 223 & 1,390 & 1.4 & 10.0 & ND & 97 \\
\hline $10 / 25 / 93$ & & 21.21 & 29 & 9.3 & 1.7 & 11 & 1.7 & 15 & 311 & .9 & 12 & ND & 84 \\
\hline $10 / 28 / 93$ & & 24.21 & 162 & 32.6 & 16.0 & 58 & 8.0 & 80 & 497 & 3.4 & 7.9 & ND & 98 \\
\hline $11 / 01 / 93$ & & 28.21 & 288 & 30.3 & 21.0 & 101 & 22.0 & 144 & 949 & .6 & 6.8 & ND & 95 \\
\hline $11 / 04 / 93$ & & 31.21 & 392 & 34.1 & 41.0 & 142 & 31.0 & 178 & 1,150 & .8 & 15.0 & ND & 102 \\
\hline $11 / 09 / 93$ & & 36.21 & 242 & 37.6 & 25.0 & 86 & 22.0 & 109 & 644 & .9 & 16.0 & ND & 74 \\
\hline $11 / 11 / 93$ & & 38.21 & 1,130 & 28.0 & 190.8 & 462 & 70.9 & 405 & 4,030 & 1.8 & 27.5 & ND & 104 \\
\hline $11 / 15 / 93$ & & 42.21 & 3,630 & 76.7 & 375.0 & 1,150 & 85.0 & 2,020 & 4,730 & ND & 13.0 & ND & 86 \\
\hline $11 / 18 / 93$ & & 45.21 & 1,100 & 32.0 & 115.0 & 381 & 85.0 & 2,160 & 3,440 & ND & 4.1 & ND & 77 \\
\hline $11 / 22 / 93$ & . & 49.21 & 1,080 & 20.9 & 72.0 & 214 & 100.0 & 593 & 5,160 & ND & 7.5 & ND & 90 \\
\hline $11 / 29 / 93$ & & 56.42 & 2,060 & 44.8 & 150.0 & 649 & 206.0 & 1,050 & 4,600 & ND & 14.0 & ND & 102 \\
\hline $12 / 03 / 93$ & & 60.46 & 935 & 39.3 & 81.0 & 256 & 68.0 & 529 & 2,380 & ND & 5.2 & ND & 97 \\
\hline $12 / 06 / 93$ & & 63.21 & 1,500 & 35.2 & 163.0 & 466 & 100.0 & 765 & 4,260 & ND & 11.0 & ND & 103 \\
\hline $12 / 09 / 93$ & & 66.21 & 1,840 & 29.8 & 138.0 & 523 & 119.0 & 1,060 & 6,170 & ND & 12.0 & ND & 104 \\
\hline \multicolumn{14}{|l|}{ TFF-EOOG-AQ } \\
\hline $10 / 26 / 93$ & & 22.38 & ND & - & ND & ND & ND & ND & - & ND & ND & ND & 93 \\
\hline $11 / 29 / 93$ & & 56.42 & ND & .0 & ND & ND & ND & ND & 35 & ND & ND & ND & 84 \\
\hline
\end{tabular}

Note: - Indicates analysis not performed.

ND: Not detected at or above limit of detection.

Total Petroleum Hydrocarbons (Window: C6 to C12).

-Chlorobenzene (QC Limits: 50 to 150). 


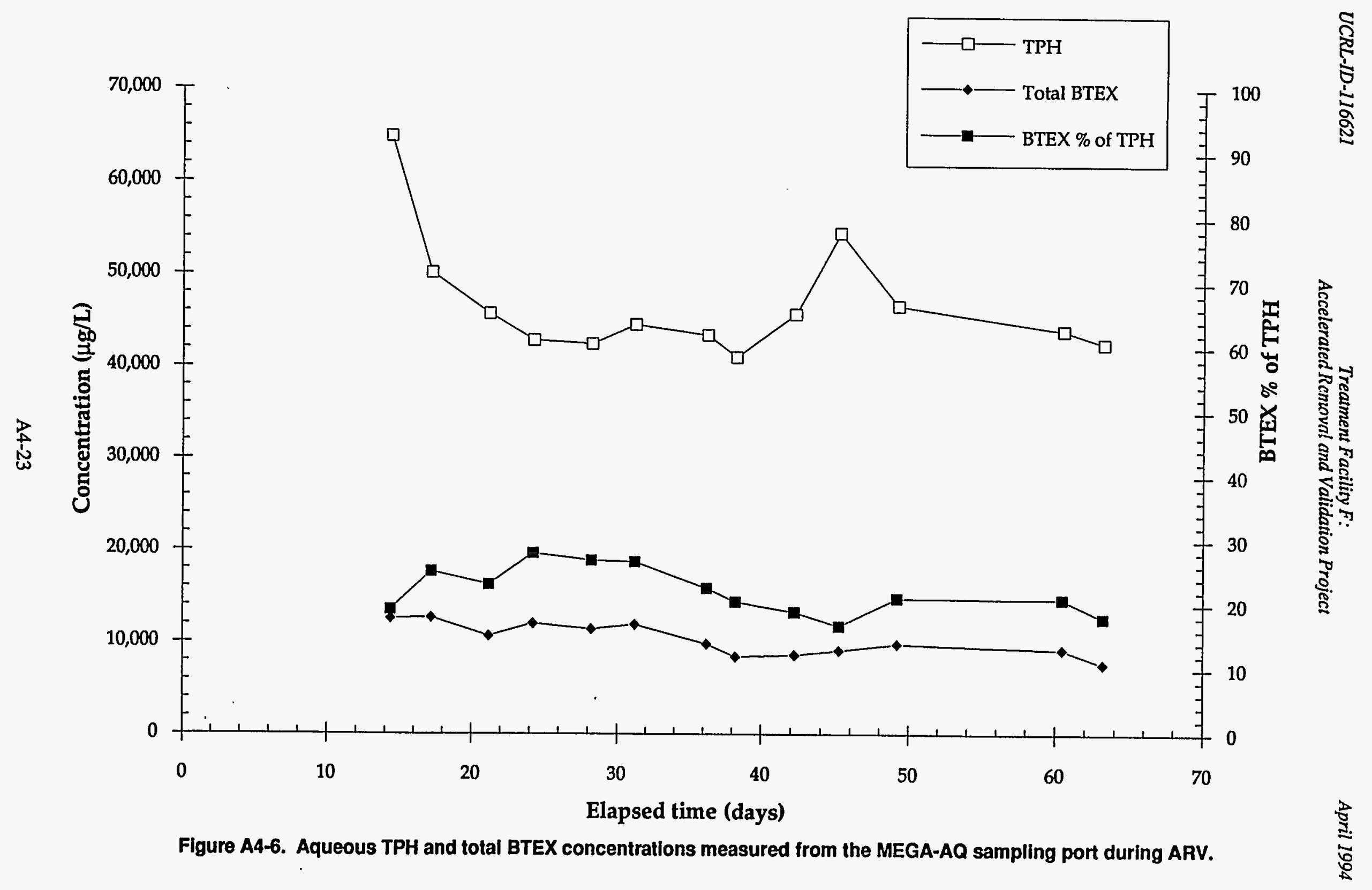




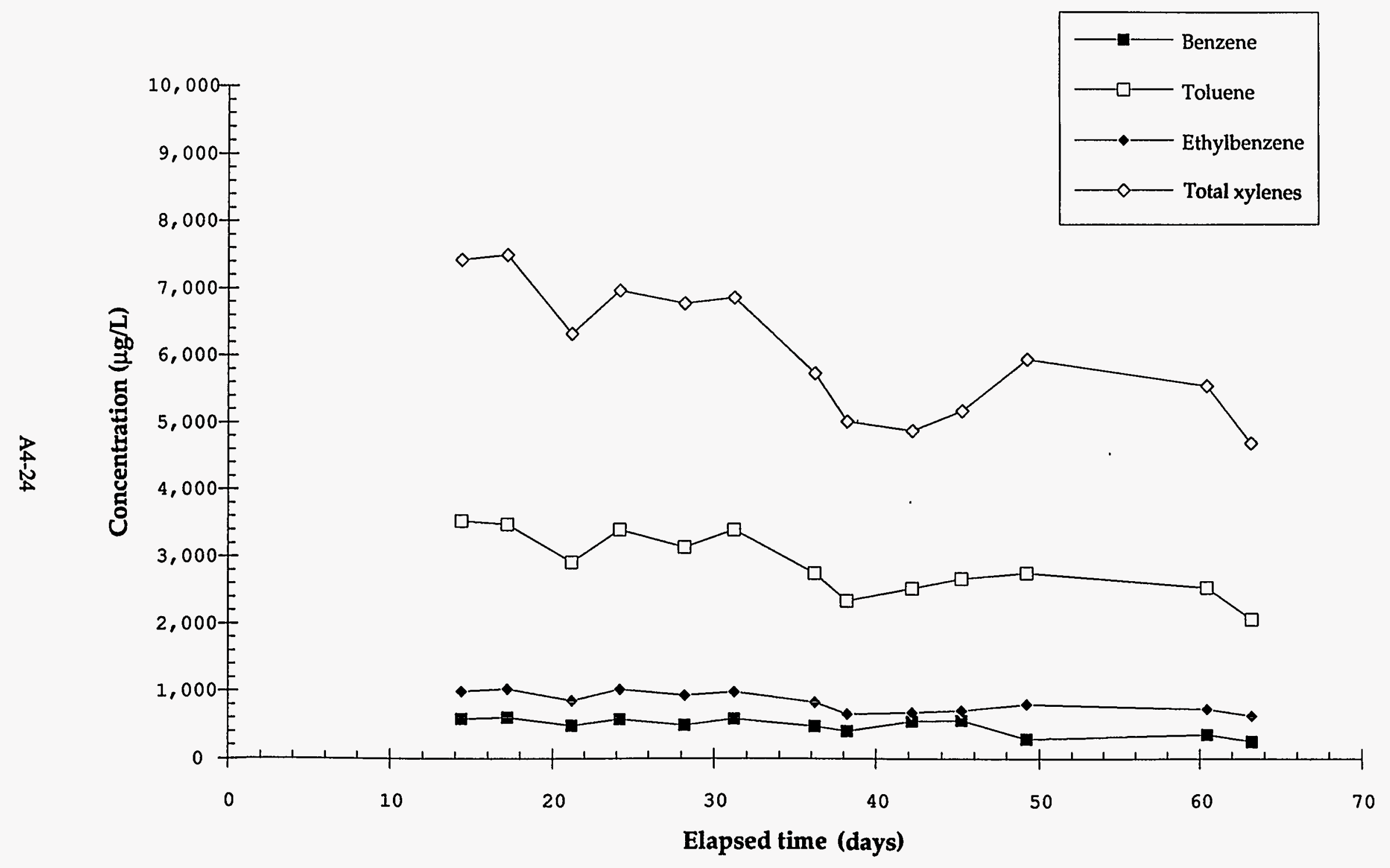

Figure A4-7. BTEX component aqueous concentrations measured from the MEGA-AQ sampling port during ARV. 
collected from ICE-IN, I006-VPR, and individual well heads contained high VOC concentrations.

ICE-IN is the designation for the internal combustion engine vapor stream influent sampling port (Fig. 3, main text). Hydrocarbon concentrations in extracted vapor were initially around 6,000 ppmv (Fig. A4-8). After 10 days of continuous extraction, hydrocarbon concentrations decreased to around 3,000 ppmv. TPH concentrations consistently averaged around $82 \%$ of total hydrocarbons. Hydrocarbon concentrations varied during the ARV project due to operational changes in the vapor treatment system. By the end of continuous operations, hydrocarbon concentrations in extracted vapor declined to around $1,000 \mathrm{ppmv}$. Table A4-8 summarizes ARV project vapor analytic results.

Total BTEX concentrations decreased from around $1,000 \mathrm{ppmv}$ to less than $300 \mathrm{ppmv}$ during the ARV project and accounted for less than 14\% of the TPH (Fig. A4-9). Concentrations of individual BTEX components were below 500 ppmv and are shown in Fig. A4-10. The largest increase was observed at about 28 days of continuous operation. Relative BTEX-component concentrations in extracted vapor were similar to those in extracted ground water.

ICE-OUT is the designation of the internal combustion engine effluent sampling port that we monitored for air compliance purposes (Fig. 3, main text). Concentrations of total BTEX were typically less than $1.0 \mathrm{ppmv}$.

CFO is the designation for the air stripper off-gas carbon treatment effluent sampling port. We monitored vapor samples from CFO daily, typically detecting less than 1 ppmv total nonmethane hydrocarbons (Fig. 3, main text).

MEGA-HC is the designation for the skimmed gasoline sampling port on the condensed fluid oil-water separator effluent (Fig. 3, main text). Table A4-9 lists the ten most prevalent components of each gasoline sample collected during the second steam pass of DUSDP and the ARV phase after September 16, 1993. The composition of condensed gasoline did not vary significantly during the ARV project.

\section{References}

U.S. Environmental Protection Agency (EPA) (1985), National Primary Drinking Water Regulations, 40 CFR Part 141, Federal Register 50:46936.

Methods for the Determination of Organic Compounds in Drinking Water, EPA-600/4-88/039, December 1988, page 46.

"Principles of Environmental Analysis," Analytic Chemistry, 55, 2210-2218, December 1983, American Chemical Society.

Standard Methods for the Examination of Water and Waste Water, 18th Edition, 1992. 

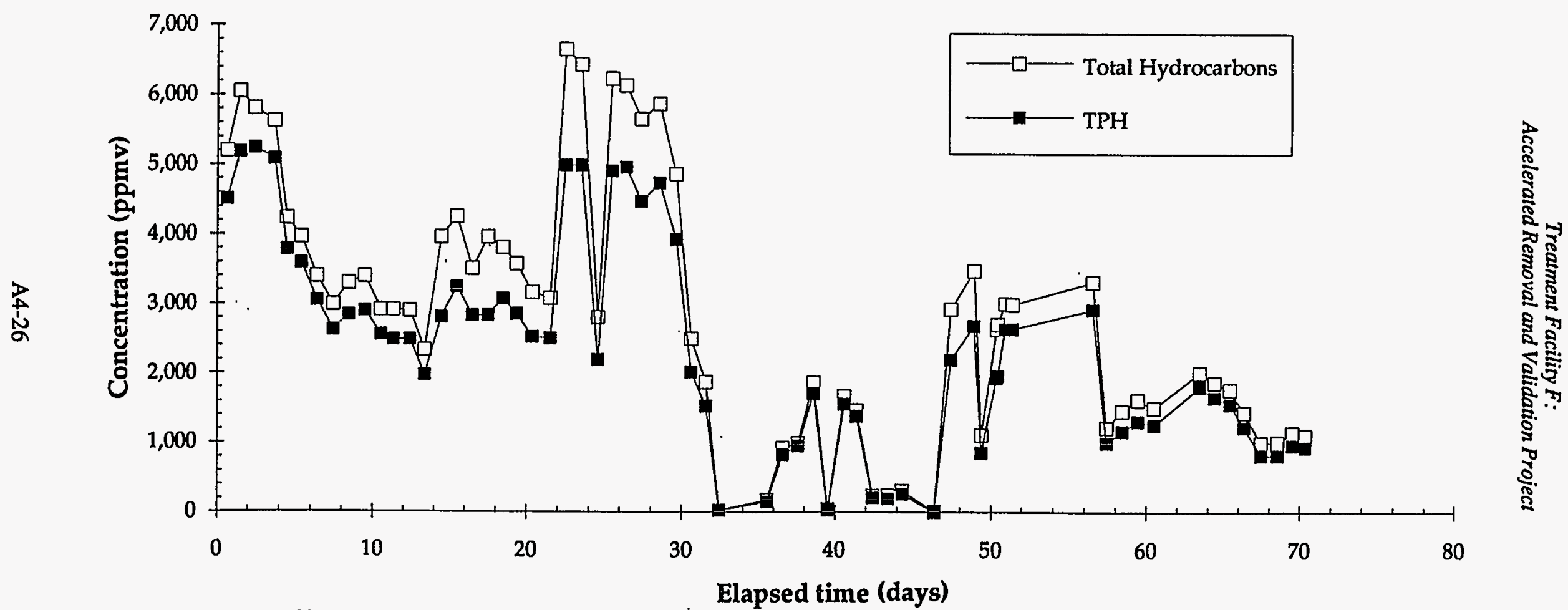

Figure A4-8. Vapor-phase total hydrocarbons and total petroleum hydrocarbons measured from the ICE-IN. 
Table A4-8. ARV project vapor analytic results.

\begin{tabular}{|c|c|c|c|c|c|c|c|c|c|c|c|}
\hline Date sampled & $\begin{array}{l}\text { Elapeed time } \\
\text { (days) }\end{array}$ & $\begin{array}{l}\text { Total BTEX } \\
\text { (ppmiv) }\end{array}$ & $\begin{array}{c}\text { BTEX } \\
\text { (\% of TPH) }\end{array}$ & $\begin{array}{l}\text { Benzene } \\
\text { (ppmv) }\end{array}$ & $\begin{array}{l}\text { Toluene } \\
\text { (ppmv) }\end{array}$ & $\begin{array}{c}\text { Ethylbenzene } \\
\text { (ppmv) }\end{array}$ & $\begin{array}{c}\text { Total xylenes } \\
\text { (ppmv) }\end{array}$ & $\begin{array}{c}\mathrm{TPH}^{*} \\
\text { (mg/L air) }\end{array}$ & $\begin{array}{c}\text { TPH* } \\
\text { (ppmv) }\end{array}$ & $\begin{array}{c}\text { HC } \\
\text { (mgl air) }\end{array}$ & $\begin{array}{l}\text { HC** } \\
\text { (ppmv) }\end{array}$ \\
\hline \multicolumn{12}{|l|}{ TFF-ICE-IN } \\
\hline $10 / 01 / 93$ & 0.67 & 8420 & 18.7 & 77.0 & 279.0 & 820 & 404.0 & 15.9 & 4,510 & 18.3 & 5,200 \\
\hline $10 / 05 / 93$ & 1.50 & 915.0 & 17.6 & 113.0 & 326.0 & 820 & 3940 & 18.3 & 5,190 & 21.3 & 6,050 \\
\hline $10 / 06 / 93$ & 246 & • & • & • & - & • & • & 18.5 & 5,250 & 20.5 & 5,810 \\
\hline $10007 / 33$ & 3.67 & 790.0 & 15.5 & 96.0 & 3080 & 680 & 3180 & 17.9 & 5,090 & 19.8 & 5,630 \\
\hline $1008 / 93$ & 4.50 & 624.0 & 16.5 & 78.0 & 225.0 & 56.0 & 265.0 & 13.4 & 3,790 & 14.9 & 4,240 \\
\hline $1009 / 93$ & 5.42 & • & • & • & • & • & • & 127 & 3,590 & 14.0 & 3,970 \\
\hline $1010 / 93$ & 6.42 & 435.0 & 14.2 & 49.0 & 157.0 & 41.0 & 1880 & 10.8 & 3,060 & 12.0 & 3,400 \\
\hline $10 / 11 / 93$ & 7.46 & 427.0 & 16.2 & 420 & 146.0 & 41.0 & 1980 & 9.3 & 2,630 & 10.6 & 3,000 \\
\hline $10 / 12 / 93$ & 8.46 & • & - & • & • & • & • & 10.3 & 2,850 & 12.0 & 3,310 \\
\hline $10 / 13 / 93$ & 9.50 & • & • & • & $\bullet$ & • & • & 10.2 & 2,910 & 12.0 & 3,400 \\
\hline $10 / 14 / 93$ & 10.54 & 427.0 & 16.7 & 39.0 & 1420 & 43.0 & 203.0 & 9.0 & 2560 & 10.3 & 2,920 \\
\hline $10 / 15 / 93$ & 11.38 & • & • & • & - & • & • & 8.8 & $2 / 490$ & 10.3 & 2,920 \\
\hline $10 / 16 / 93$ & 12.42 & - & - & - & - & - & - & 8.8 & 2,490 & 10.3 & 2,910 \\
\hline $10117 / 93$ & 13.42 & • & • & • & $\bullet$ & • & • & 7.0 & 1,980 & 8.2 & 2,340 \\
\hline $10 / 18 / 93$ & 18.50 & 324.0 & 11.5 & 39.0 & 1080 & 300 & 147.0 & 9.9 & 2,820 & 14.0 & 3,970 \\
\hline $10 / 19 / 93$ & 15.50 & • & - & - & • & • & • & 11.5 & 3,250 & 15.0 & 4,260 \\
\hline $10 / 20 / 93$ & 16.50 & - & - & - & • & - & - & 10.0 & 2840 & 124 & 3,520 \\
\hline $10 / 21 / 93$ & 17.50 & • & - & - & - & - & - & 11.0 & 2840 & 14.0 & 3,970 \\
\hline $10 / 22 / 93$ & 18.50 & 268.0 & 8.7 & 28.0 & 960 & 250 & 1190 & 10.8 & 3,080 & 13.5 & 3,820 \\
\hline $10 / 23 / 93$ & 19.38 & • & - & • & • & • & $\bullet$ & 10.1 & 28860 & 126 & 3,580 \\
\hline $10 / 24 / 93$ & 20.38 & • & • & • & • & • & • & 8.9 & 2530 & 11.0 & 3,170 \\
\hline $10 / 25 / 93$ & 21.50 & 6420 & 25.6 & 41.0 & 183.0 & 69.0 & 349.0 & 8.8 & 2,510 & 10.9 & 3,090 \\
\hline $10 / 26 / 93$ & 22.50 & • & • & • & $\bullet$ & • & • & 18.0 & 5,000 & 23.5 & 6,660 \\
\hline $10 / 27 / 93$ & 23.50 & • & • & • & • & • & • & 17.6 & 5,000 & 227 & 6,440 \\
\hline $10 / 28 / 93$ & 24.58 & $\mathbf{2 3 0 . 0}$ & 19.5 & 32.0 & 1420 & 140 & 2120 & 7.7 & 2,200 & 9.9 & 2,810 \\
\hline $10 / 29 / 93$ & 25.50 & • & • & • & • & • & • & 17.3 & 4,920 & 220 & 6,240 \\
\hline $10 / 30 / 93$ & 26.38 & • & - & - & - & - & - & 17.5 & 4,970 & 22.6 & 6,140 \\
\hline 10/31/93 & 27.38 & $\bullet$ & $\bullet$ & • & $\bullet$ & • & • & 15.8 & $4 A 80$ & 19.9 & 5,660 \\
\hline $11101 / 93$ & 28.54 & $1,540.0$ & 32.5 & 89.0 & 487.0 & 167.0 & 8000 & 16.7 & 4,740 & 20.7 & 5,880 \\
\hline $11 / 02 / 93$ & 29.13 & • & • & • & $\bullet$ & • & • & 13.8 & 3,930 & 172 & 4,870 \\
\hline $11 / 03 / 93$ & 30.58 & • & • & • & • & • & • & 7.1 & 2020 & 8.8 & 2,500 \\
\hline $11 / 04 / 93$ & 31.58 & 465.0 & 30.4 & 29.0 & 1440 & 500 & 2420 & 5.4 & 1,530 & 6.6 & 1,880 \\
\hline $11 / 05 / 93$ & 3246 & • & • & • & • & • & • & 0.1 & 36 & 0.1 & 37 \\
\hline $11 / 08 / 93$ & 35.58 & 12.0 & 7.9 & ND & 4.4 & ND & 7.1 & 0.5 & 151 & 0.6 & 178 \\
\hline $11 / 09 / 33$ & 36.58 & 229.0 & 27.5 & 11.0 & 62.0 & 31.0 & 1260 & 29 & 832 & 3.3 & 926 \\
\hline 11/20/93 & 37.58 & • & • & • & • & • & • & 3.4 & 960 & 3.5 & 993 \\
\hline $11 / 11 / 93$ & 38.63 & 356.0 & 20.8 & 38.0 & 158.0 & 280 & 131.0 & 6.0 & 1,710 & 6.6 & 1,870 \\
\hline $11 / 12 / 93$ & 39.54 & $\bullet$ & • & • & • & • & • & 0.1 & 36 & 0.1 & 39 \\
\hline $11 / 13 / 93$ & 40.58 & - & - & - & - & • & • & 5.5 & 1,560 & 5.9 & 1,670 \\
\hline $11114 / 93$ & $\$ 1.38$ & • & • & • & • & • & • & 4.9 & 1,390 & 5.2 & 1,470 \\
\hline $11 / 15 / 93$ & 22.46 & 39.0 & 18.3 & 11.0 & 13.0 & 2.7 & 11.4 & 0.7 & 213 & 0.8 & 235 \\
\hline $11 / 16 / 93$ & 13,12 & 23.0 & 11.4 & 3.5 & 7.6 & 1.6 & 9.9 & 0.7 & 202 & 0.8 & 240 \\
\hline $11 / 16 / 93$ & 43.16 & 72.0 & 5.4 & 5.7 & 25.0 & 7.1 & 35.0 & 4.7 & 1,340 & 5.2 & 1,480 \\
\hline $11 / 16 / 93$ & $\$ 3.50$ & 70.0 & 4.3 & 6.4 & 21.0 & 6.2 & 36.0 & 5.7 & 1,610 & 6.3 & 1,790 \\
\hline $11 / 16 / 33$ & 33.54 & 155.0 & 14.2 & 23.0 & 65.0 & 120 & 55.0 & 3.8 & 1,090 & 4.3 & 1,210 \\
\hline $11 / 16 / 93$ & 43.58 & 92.0 & 5.9 & 17.0 & 46.0 & 7.0 & 220 & 5.4 & 1,550 & 5.9 & 1,690 \\
\hline $11 / 16 / 93$ & $\$ 3.63$ & 124.0 & 6.3 & 20.0 & 620 & 7.8 & 32.4 & 6.9 & 1,960 & 7.5 & 2,120 \\
\hline $11 / 16 / 93$ & $\$ 3.67$ & 52.0 & 6.4 & 13.0 & 25.0 & 2.7 & 11.1 & 2.9 & 812 & 3.1 & 878 \\
\hline $11 / 16 / 93$ & 43.75 & 16.0 & 5.0 & 4.0 & 10.0 & ND & 18 & 1.1 & 319 & 1.3 & 356 \\
\hline $11 / 16 / 93$ & 43.23 & 16.0 & 27 & 3.4 & 84 & 13 & 32 & 21 & 586 & 23 & 646 \\
\hline $11 / 16 / 93$ & 13.92 & 13.0 & 28 & 3.6 & 6.8 & ND & 21 & 1.6 & 165 & 1.9 & 526 \\
\hline $11 / 17 / 93$ & 42.04 & • & • & • & • & • & - & 1.9 & 545 & 2.2 & 616 \\
\hline $11 / 17 / 93$ & $\$ 1.17$ & • & • & • & • & • & • & ND & ND & 0.0 & 12 \\
\hline $11 / 17 / 93$ & $\$ 1.33$ & 6.1 & 23 & 1.4 & 3.5 & ND & 1.2 & 0.9 & 266 & 1.1 & 310 \\
\hline 11/19/93 & 46.38 & • & • & • & • & • & • & ND & ND & 0.0 & 12 \\
\hline $11 / 20 / 93$ & S7.38 & - & - & - & • & - & - & 7.8 & 2,200 & 10.3 & 2,930 \\
\hline $11 / 21 / 93$ & 18.08 & • & • & • & • & - & • & 9.5 & 2,690 & 12.3 & 3,490 \\
\hline $11 / 22 / 93$ & 49.38 & 22.0 & 4.9 & ND & 14.0 & 5.1 & 22.6 & 3.1 & 865 & 3.9 & 1,120 \\
\hline $11 / 22 / 93$ & 49.16 & • & • & • & • & • & • & 0.3 & 86 & 0.3 & 85 \\
\hline $11 / 22 / 93$ & 49.54 & ND & • & ND & ND & ND & ND & ND & ND & 0.1 & 16 \\
\hline $11 / 22 / 93$ & 49.63 & 163.0 & 10.4 & 6.8 & 56.0 & 18.0 & 820 & 5.5 & 1,560 & 6.8 & 1,930 \\
\hline $11 / 22 / 93$ & 99.67 & 960.0 & 34.7 & 41.0 & 3540 & 93.0 & 4720 & 9.8 & 2,770 & 9.7 & 2,760 \\
\hline $11 / 22 / 93$ & 49.03 & 259.0 & 37.4 & 14.0 & 1000 & 230 & 1220 & 24 & 693 & 26 & 729 \\
\hline $11 / 23 / 93$ & 50.04 & 68.0 & 3.3 & 28 & 24.0 & 80 & 33.0 & 7.2 & 2,050 & 10.0 & 2840 \\
\hline $11 / 23 / 93$ & 50.21 & 820 & 3.9 & 4.6 & 28.0 & 100 & 400 & 7.3 & 2,080 & 10.0 & 2,830 \\
\hline $11 / 23 / 93$ & 50.33 & 78.0 & 4.0 & 4.0 & 28.0 & 88 & 36.7 & 6.8 & 1,940 & 9.3 & 2,640 \\
\hline $11 / 23 / 93$ & 50.42 & • & • & • & • & • & • & 6.9 & 1,970 & 9.6 & 2,710 \\
\hline $11 / 23 / 93$ & 50.22 & - & - & - & • & - & - & 6.6 & 1,870 & 9.1 & 2,570 \\
\hline $11 / 23 / 93$ & 50.42 & • & • & • & • & • & $\bullet$ & 7.0 & 1,990 & 9.7 & 2,740 \\
\hline $11 / 23 / 93$ & 50.288 & 485.0 & 18.4 & 520 & 218.0 & 380 & 177.0 & 9.3 & 2,640 & 10.6 & 3,010 \\
\hline
\end{tabular}


Table A4-8 (continued)

\begin{tabular}{|c|c|c|c|c|c|c|c|c|c|c|c|}
\hline Date ampled & $\begin{array}{l}\text { Elapsed time } \\
\text { (dayn) }\end{array}$ & $\begin{array}{l}\text { Total BTEX } \\
\text { (PPmv) }\end{array}$ & $\begin{array}{c}\text { BTEX } \\
\text { ( } \% \text { of TPH) }\end{array}$ & $\begin{array}{l}\text { Benzene } \\
\text { (ppur) }\end{array}$ & $\begin{array}{l}\text { Toluene } \\
\text { (ppmv) }\end{array}$ & $\begin{array}{c}\text { Ethylbenzene } \\
\text { (ppmv) }\end{array}$ & $\begin{array}{c}\text { Total xylenes } \\
\text { (ppur) }\end{array}$ & $\begin{array}{c}\text { TPH* } \\
\text { (mg/L air) }\end{array}$ & $\begin{array}{c}\text { TPH* } \\
\text { (ppmv) }\end{array}$ & $\begin{array}{c}\text { HC** } \\
\text { (mg/L air) }\end{array}$ & $\begin{array}{l}\text { HC+* } \\
\text { (ppmv) }\end{array}$ \\
\hline \multicolumn{12}{|c|}{ TFF-ICE-IN (Cont'd) } \\
\hline $11 / 24 / 93$ & 51.38 & 469.0 & 17.8 & 52.0 & 210.0 & 360 & 1700 & 9.3 & 2,640 & 10.6 & 3,000 \\
\hline $11 / 29 / 93$ & 36.54 & 425.0 & 18.6 & 33.0 & 151.0 & 380 & 2000 & 10.3 & 2,920 & 11.7 & 3,320 \\
\hline $11 / 30 / 93$ & 57.46 & • & - & - & - & - & 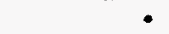 & 3.5 & 1,000 & 4.3 & 1,220 \\
\hline 12 1201/93 & 58.46 & • & - & • & • & • & • & 4.1 & 1,170 & 5.1 & 1,460 \\
\hline $12 / 202 / 33$ & 59.46 & 200.0 & 15.3 & 17.0 & 81.0 & 17.0 & 84.0 & 4.6 & 1,310 & 5.7 & 1,620 \\
\hline $12503 / 93$ & 60.50 & • & - & - & - & - & - & 4.5 & 1260 & 5.3 & 1,510 \\
\hline $12006 / 93$ & 63.46 & 229.0 & 127 & 16.0 & 96.0 & 2000 & 990 & 6.4 & 1,810 & 7.1 & 2,010 \\
\hline $1207 / 93$ & 6246 & • & • & - & - & • & - & 5.8 & 1,660 & 6.6 & 1,870 \\
\hline 12 120893 & 65.46 & • & - & - & - & • & • & 5.5 & 1,560 & 5.2 & 1,770 \\
\hline $1209 / 93$ & 66.38 & 218.0 & 17.7 & 21.0 & 85.0 & 2000 & 920 & 4.3 & 1,230 & 5.1 & 1,440 \\
\hline 12/10/53 & 67.46 & 107.0 & 13.0 & 6.4 & 38.0 & 120 & 51.0 & 29 & 825 & 3.5 & 1,000 \\
\hline $12 \pi 1 / 33$ & 68.54 & - & - & - & - & - & - & 29 & 827 & 3.6 & 1,010 \\
\hline $12 / 12 / 33$ & 6.54 & • & • & • & - & • & • & 3.4 & $\mathbf{9 7 0}$ & 4.0 & 1,150 \\
\hline $12 / 13 / 83$ & 70.38 & 920 & 9.9 & 8.9 & 27.0 & 120 & 44.0 & 3.3 & 934 & 3.9 & 1,110 \\
\hline \multicolumn{12}{|l|}{ TFF-CFO } \\
\hline $10 / 8 / 93$ & 1.33 & ND & - & ND & ND & ND & ND & ND & ND & 0.004 & 1.0 \\
\hline \multicolumn{12}{|l|}{ TFF-ICE-OUT } \\
\hline $10 / 08 / 93$ & $\$ .33$ & 1.0 & 35.7 & 1.0 & ND & ND & ND & 0.0 & 2.8 & 0.009 & 27 \\
\hline \multicolumn{12}{|l|}{ TFF-GIW-815 } \\
\hline $11 / 16 / 93$ & 13.40 & ND & - & ND & ND & ND & ND & ND & ND & 0.040 & 12 \\
\hline $11 / 16 / 93$ & 43.48 & • & $\bullet$ & • & • & • & - & 4.0 & 1,140 & 4.4 & 1,250 \\
\hline $11 / 16 / 93$ & $\mathbf{4 3 . 5 4}$ & 218.0 & 13.3 & 20.0 & 1240 & 15.0 & 59.0 & 5.8 & 1,640 & 6.1 & 1,730 \\
\hline $11 / 16 / 93$ & 13.58 & $1,200,0$ & 20.0 & 34.0 & 582.0 & 110.0 & 470.0 & 21.0 & 6,000 & 23.0 & 6,570 \\
\hline $11 / 16 / 93$ & $\$ 3.63$ & 554.0 & 8.6 & 28.0 & 350.0 & 210 & 155.0 & 23.0 & $6 A 30$ & 25.0 & 6,980 \\
\hline $11 / 16 / 93$ & $\$ 3.67$ & 179.0 & 3.2 & 8.9 & 960 & 8.9 & 65.0 & 20.0 & 5,650 & 24.0 & 6,760 \\
\hline \multicolumn{12}{|l|}{ TFF-GIW-820 } \\
\hline $11 / 16 / 93$ & $\$ 3.38$ & 2.4 & 4.1 & ND & 21 & ND & 1.3 & 0.2 & 59 & 0.25 & 70 \\
\hline $11 / 16 / 93$ & 13.39 & 1.1 & 6.5 & ND & 21 & ND & ND & 0.1 & 17 & 0.09 & 24 \\
\hline \multicolumn{12}{|c|}{ TFF-GWW-820-Lower } \\
\hline $11 / 22 / 93$ & 19.36 & 11.0 & - & ND & 7.1 & ND & 42 & - & - & - & - \\
\hline $11 / 22 / 93$ & 49.41 & ND & - & ND & ND & ND & ND & - & - & - & - \\
\hline $11 / 22 / 93$ & 49.42 & 3960 & - & 18.0 & 1850 & 37.0 & 157.0 & • & $\cdot \bullet$ & - & • \\
\hline $11 / 22 / 33$ & 49.44 & 154.0 & 14.1 & 7.7 & 68.0 & 15.0 & 640 & 3.8 & 1,090 & 4.3 & 1,220 \\
\hline $11 / 22 / 93$ & 49.46 & • & • & • & • & • & • & 7.4 & 2,090 & 8.1 & 2,300 \\
\hline $11 / 22 / 93$ & 49.51 & 13.0 & - & ND & 7.9 & ND & 5.0 & $\bullet$ & $\bullet$ & • & • \\
\hline $11 / 22 / 93$ & 49.54 & 5.0 & - & ND & ND & ND & 5.0 & - & - & - & - \\
\hline \multicolumn{12}{|c|}{ TFF-GIW-820-U UpeT } \\
\hline $11 / 22 / 13$ & 49.36 & 8.5 & 7.9 & ND & ND & 4.8 & 3.7 & 0.40 & 108 & 0.49 & 140 \\
\hline $11 / 22 / 93$ & 49.40 & $\bullet$ & • & • & • & - & • & 0.70 & 194 & 0.87 & 248 \\
\hline $11 / 22 / 93$ & 49.42 & - & - & - & - & - & - & 1.90 & 547 & 250 & 720 \\
\hline $11 / 20 / 93$ & 29.44 & $\bullet$ & • & • & - & $\bullet$ & $\bullet$ & 5.40 & 1,520 & 7.00 & 1,990 \\
\hline $11 / 22 / 93$ & 49.46 & 138.0 & 7.1 & 3.4 & 48.0 & 17.0 & 70.0 & 6.90 & 1,950 & 8.90 & 2,530 \\
\hline $11 / 22 / 93$ & 49.49 & • & - & - & • & • & • & 0.10 & 23 & 0.10 & 28 \\
\hline $11 / 22 / 93$ & 49.50 & • & • & - & - & - & - & 0.10 & 30 & 0.11 & 31 \\
\hline $11 / 22 / 93$ & 49.52 & • & • & • & • & • & • & ND & ND & ND & ND \\
\hline $11 / 22 / 93$ & 49.54 & ND & 0.0 & ND & ND & ND & ND & 0.10 & 29 & 0.12 & 34 \\
\hline $11 / 22 / 93$ & 49.57 & - & - & • & • & - & • & 0.30 & 97 & 0.35 & 98 \\
\hline $11 / 22 / 93$ & 49.60 & ND & 0.0 & ND & ND & ND & ND & 0.10 & 31 & 0.11 & 31 \\
\hline $11 / 22 / 93$ & 49.65 & • & • & • & • & • & • & 0.10 & 31 & 0.11 & 32 \\
\hline
\end{tabular}

- Indicates analysis not performed.

ND: Not detected at or above linit of detection.

-Total petroleum hydrocarbons (Window: $\mathrm{C6}$ to C12).

"Total hydrocasbons (Window: C1 to C12). 

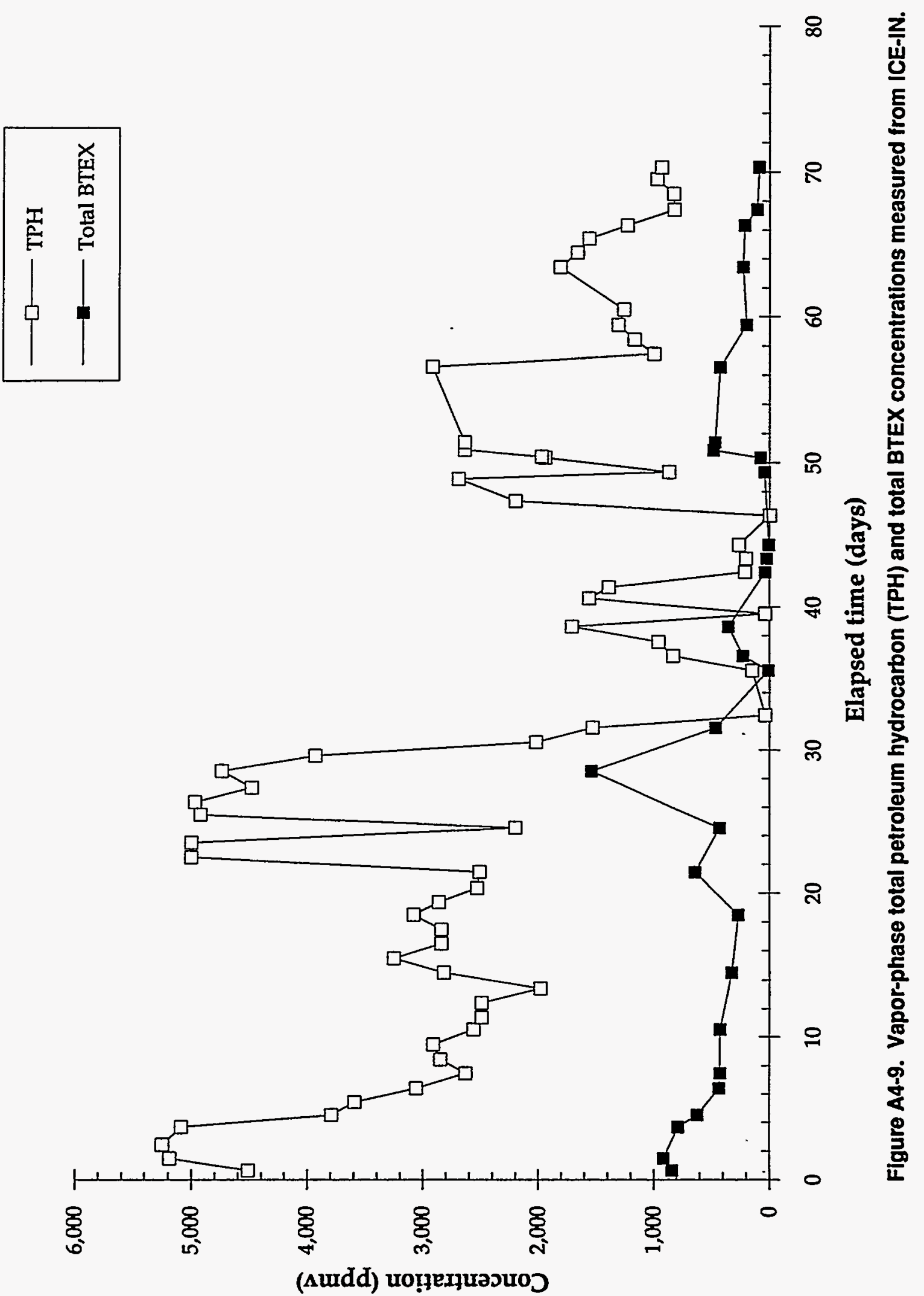


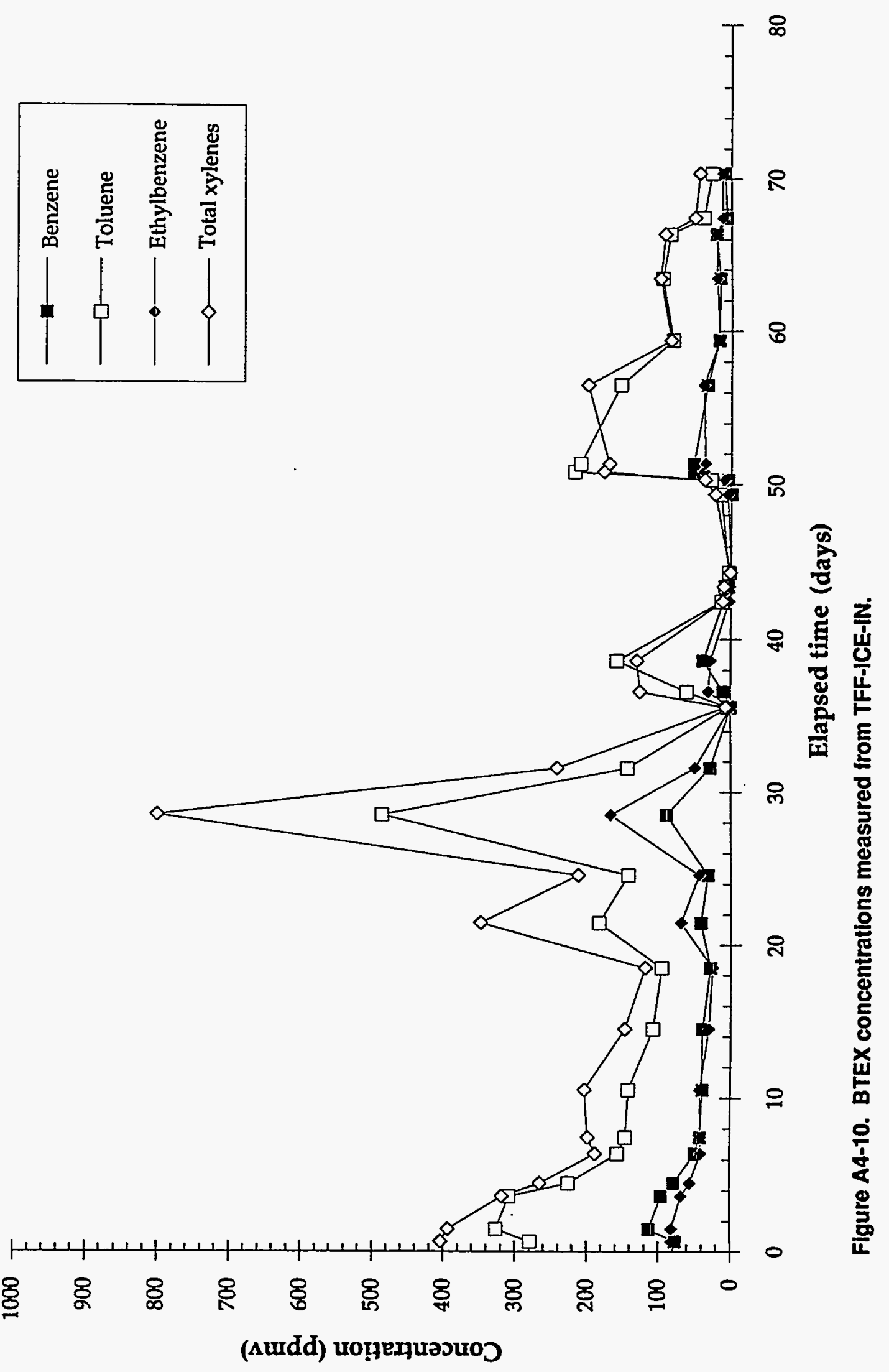


Table A4-9 - Ten largest identified peaks by GCIMS of MEGA-HC condensed vapor.

\begin{tabular}{|c|c|c|c|c|c|c|c|c|c|c|}
\hline \multirow{3}{*}{$\begin{array}{l}\text { Retention time } \\
\text { (min) }\end{array}$} & \multirow{3}{*}{$\begin{array}{c}\text { Tentatively } \\
\text { identified compounds }\end{array}$} & \multicolumn{9}{|c|}{ Dates sampled } \\
\hline & & $5 / 25 / 93$ & $5 / 27 / 93$ & $6 / 3 / 93$ & $6 / 15 / 93$ & $6 / 17 / 93$ & $6 / 21 / 93$ & $9 / 16 / 93$ & $10 / 13 / 93$ & $10 / 25 / 93$ \\
\hline & & (\%) & (\%) & (\%) & (\%) & (\%) & (9) & (\%) & (\%) & (\%) \\
\hline 1.97 & 2-methyl pentane & 3.4 & & & & & & & & \\
\hline 2.38 & Methyl cyclopentane & 3.1 & & & & & & & & \\
\hline 2.77 & 2-methyl hexane & 6.1 & 3.6 & & & & & & & \\
\hline 2.88 & 3-methyl hexane & 4.1 & 2.4 & & & & & & & \\
\hline 3.19 & Heptane & 3.6 & & & & & & & & \\
\hline 4.22 & Toluene & 12 & 11 & 3.9 & 3.2 & 2.8 & & & & \\
\hline 4.77 & Octane & & 2.4 & & & & & & & \\
\hline 5.87 & Ethyl benzene & & 3.2 & 3.5 & 3.5 & 3.1 & & & & \\
\hline 6.08 & m,p-xylene & 15 & 12 & 11 & 9.0 & 11 & 4.0 & 6.8 & 4.6 & 4.0 \\
\hline 6.49 & o-xylene & 4.2 & 4.9 & 4.9 & $\mathbf{5 . 0}$ & 4.6 & 2.7 & 2.8 & & \\
\hline 7.55 & Propylbenzene & & & & 2.8 & & & & & \\
\hline 7.70 & 1-ethyl-2-methyl benzene & 4.8 & 6.6 & 4.5 & & & 5.2 & 7.4 & 6.4 & 5.8 \\
\hline 7.82 & 1,3,5-trimethyl benzene & & 2.6 & 7.0 & 8.8 & 9.7 & & 3.0 & & \\
\hline 8.28 & 1,2,4-trimethyl benzene & & 7.2 & 2.7 & 7.0 & 11 & 9.6 & 11.3 & 10.3 & 9.6 \\
\hline 8.75 & 1,2,3-trimethyl benzene & 3.8 & & 9.1 & 4.3 & 4.6 & 2.0 & 3.7 & 3.7 & 3.4 \\
\hline 8.78 & 1-ethyl-3-methyl benzene & & & 4.5 & & & & 9.6 & & \\
\hline 9.22 & 1-methyl-3-propyl benzene & & & 3.7 & 3.6 & 3.7 & & 4.1 & 4.3 & 3.9 \\
\hline 9.80 & 1-methyl-2-(1-methylethyl)benzene & & & & & 3.0 & 3.0 & 2.6 & 3.0 & 3.0 \\
\hline 10.77 & 1, 2,3,5-tetramethylbenzene & & & & & & 2.3 & & & \\
\hline 10.83 & 1-methyl-2-(2-propenyl)benzene & & & & & & & 2.9 & 3.8 & 3.9 \\
\hline 11.28 & C4 alkylbenzene & & & & 26 & & 1.9 & & & \\
\hline 11.40 & Naphthalene & & & & & 2.8 & 3.0 & 2.9 & 3.8 & 4.1 \\
\hline 13.00 & 2-methylnaphthalene & & & & & & 2.7 & & 3.4 & 4.1 \\
\hline 13.24 & 1-methylnaphthalene & & & & & & 2.7 & & 3.4 & 4.1 \\
\hline
\end{tabular}




\title{
Appendix 5
}

\section{Modeling of ARV Sparging and Electrical Heating Using NUFT and an Ohmic Heating Module, and Related Laboratory Measurements}

\author{
(C. R. Carrigan and J. J. Nitao, Earth Science Division, LLNL)
}

\section{Introduction}

The Dynamic Underground Stripping Demonstration Project (DUSDP) incorporated several remediation technologies simultaneously to mobilize and extract contaminants from both highand low-permeability formations in the near-surface hydrologic regime. Understanding how steam injection into soils and ohmic heating (produced by passing electrical currents through the soils) interact to affect the rate of contaminant extraction has been one goal of the project. This understanding is a prerequisite to generalizing the technique for other cleanup sites. This understanding is also necessary if the efficiency of the technique's application at a specific site is to be maximized. To develop a predictive/diagnostic capability, we produced a generalized numerical three dimensional (3D) model of the in situ ohmic or joule heating process, which has been coupled to an existing 3D simulator for nonisothermal porous flow and transport (NUFT). This combined joule heating/hydrologic model permits a full simulation of the stripping process in a hydrologic medium of arbitrary complexity.

The ohmic heating program is modular and may either be executed with NUFT or in the stand-alone mode. In stand-alone operation, the program can be used to develop a basic understanding of how electrode placement can affect the current flow and, hence, the volumetric heating rate in a volume having a prescribed electrical conductivity distribution. In the absence of detailed hydrologic, geologic, and electrochemical information, this approach may be preferred during the initial planning phases of a heating and extraction facility. The module can be used to:

- Obtain preliminary estimates of the voltage and current demands on the power supply.

- Test various assumptions on the apometric localization of ohmic heating around electrodes.

- Obtain the effect on joule heating caused by changing electrode placement in either the horizontal or vertical coordinates.

- Determine the best electrode configuration for maximizing the heating rate in specific parts of the hydrologic system. 
The module also simulates the application of either single phase or multiphase electrical power to the electrodes, a factor that must be considered in designing and costing an electrical system for a site.

As a module functioning interactively with the NUFT hydrologic program, a variety of complex, mutually dependent processes can be investigated. For example, the injection of steam at a well can affect the electrical conductivity through changes in both the saturation and temperature of the soil. Another phenomenon of interest is the formation of a high resistivity zone around electrodes owing to the drying out of that soil near the electrodes. The formation of such a zone or skin of high electrical resistivity around the electrodes will tend to redistribute ohmic dissipation so that more heating occurs locally near the electrodes rather than uniformly throughout the soil volume under consideration. Another concern that must be addressed during the operations stage is the schedule for injecting steam and for ohmic heating. Because large amounts of energy are expended to heat the soil during the stripping operation, it is desirable to determine the most cost-effective schedule for the steam stripping/ohmic heating process for a particular site.

\section{Air Sparging at TFF}

The first problem modeled in support of the ARV phase of operations at Treatment Facility F (TFF) involved the isothermal modeling of air injection and vapor extraction in wells at the gas pad site. For this, NUFT alone was used to develop an isothermal model. The problem involved injecting air in the lower steam zone (LSZ) at an injection well while attempting to remove the air at an extraction well some distance away and downdip $\left(8^{\circ}\right)$. In addition to the downdip tilt between the injection and extraction wells, there was an additional $4^{\circ}$ tilt at right angles to the first tilt, placing the injection well downdip from a monitoring well (see Fig. A5-1).

The LSZ was simulated by a tilted rectangular layer $(140 \mathrm{~m}(1) \times 126 \mathrm{~m}(\mathrm{w}) \times 4 \mathrm{~m}(\mathrm{~d}))$ that was typically gridded by more than 2,300 3D elements $(33 \times 22 \times 4)$. The material properties of the layer were chosen to correspond to those of the LSZ. High permeability is a major feature of this zone. Hydrostatic pressure distributions were applied at the sides of the layer to simulate a farfield pressure distribution in the tilted LSZ below the water table. The injection and extraction wells were modeled by assigning a mass injection rate for the injection well and a subatmospheric pressure to the extraction well.

Figure A5-1 shows plan views of the air plume at different times following initiation of the injection process. As its growth proceeded, the plume migrated up and across the tilted layer along the gradient of elevation. Irrespective of the extraction pumping rates downdip at the extraction well, no air from the plume was ever drawn down to the extraction well. This numerical prediction was borne out by the actual tests in which injected air in the LSZ did not reach the extraction well. Some air from the injection well, i.e., the fringe of the plume, did reach the monitoring well about an hour after injection commenced. This behavior was also supported by the observations at the gas pad monitoring well.

These simulations of air sparging indicate that geologic control, i.e., the tilting of the formation, dominated in determining the path of the injected plume. Even very high ( $214 \mathrm{gal} / \mathrm{m}$ ) simulated pumping rates from the extraction well did not significantly influence the plume's direction. Considering that the tilt of the LSZ can only be considered at worst to be moderate, 


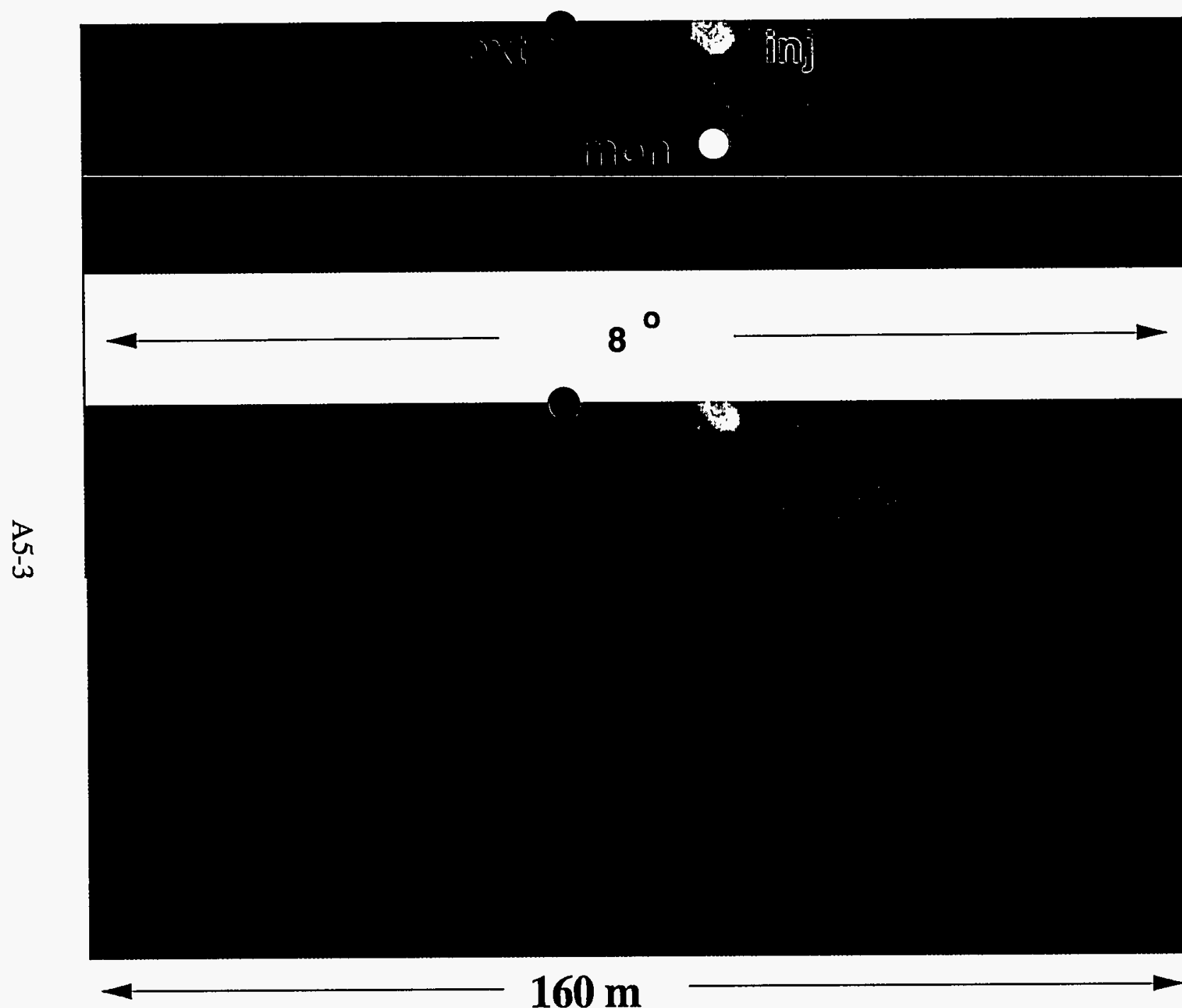

Figure A5-1. Plan view of air sparging model. Air is injected into 4-m layer at the injection well, and water is extracted at the well marked "ext." Irrespective of extraction rates, the air plume follows updip, away from the extraction well. 
we conclude that very careful placement of injection and extraction wells is necessary if the air sparging process is to be successful for many hydrologic environments. Otherwise, air injection without effective extraction may spread contamination well beyond its original borders.

\section{Ohmic Heating Simulations}

With the ohmic heating module coupled to the NUFT hydrologic model, a variety of heating models was investigated. The main thrust of the modeling effort was to investigate the effect of different electrode configurations in a multilayered hydrologic model that also included the temperature, saturation, and medium dependent variations in the electrical conductivity, which are typically associated with the layering of different soil types. The dependence of the electrical conductivity on the temperature, saturation, and soil type was evaluated using the model of Waxman and Smits (1968).

A major change in the ARV heating system was the introduction of long electrodes (approx. $60 \mathrm{ft}$ ) that extended across the upper steam zone (USZ), clay confining layer, and LSZ. The original electrodes used in the DUSDP were emplaced only in the clay confining layer. Power levels maintained during the ARV phase were either comparable or less than those used during the DUSDP. In the ARV phase, ohmic heating of the layering resulted in much lower heating rates compared to the DUSDP experience, which could not be explained by differences in power input alone. In addition, large amounts of vapor were removed from an extraction well located centrally between the long electrodes (refer to Section IV in the main report).

A series of different ohmic heating models were examined. The models were based upon the five-layer hydrologic model illustrated in Figure A5-2. This five-layer model includes the three layers of greatest interest to dynamic stripping: an unsaturated, permeable sandy-clay soil (labeled USZ in Fig. A5-4); a saturated, low-permeability clay layer (CON) directly beneath; and another permeable, but saturated, sandy-clay layer (LSZ) beneath the clay layer. In addition, this three-layer arrangement is bounded at the top and bottom by highly conductive and impermeable clay layers whose existence is indicated by well logs.

One effect of short versus long electrodes in this five-layer model is illustrated in the vertical profile of volumetric heating across the five layers in Figure A5-3. Assuming the same power dissipation in both cases, short electrodes in the central clay layer produce a much higher level of volumetric heating (dotted line) than do the longer electrodes (solid line). With the longer electrodes, more power is lost in heating other layers, especially the top clay layer, which is penetrated by the long electrodes. For constant power levels, long electrodes are less capable of focusing ohmic heating into specific layers such as the central clay layer.

The lack of specificity in heating associated with the long electrodes is exacerbated when steam is produced at the electrodes and this steam is free to flow into the layered formation. The value of the ohmic heating technique lies mainly in its ability to heat low-permeability layers that cannot be effectively flushed or heated by steam injection. Thus, when steam is produced at an electrode, two undesirable things occur. First, electrical energy is lost to steam that cannot effectively heat the low permeability layers. Second, the steam flows to other more permeable layers, heating them and also enhancing their electrical conductivity, so that more current flows through them. When vapor extraction from the unsaturated zone is also taking place, the steam 
Sat:

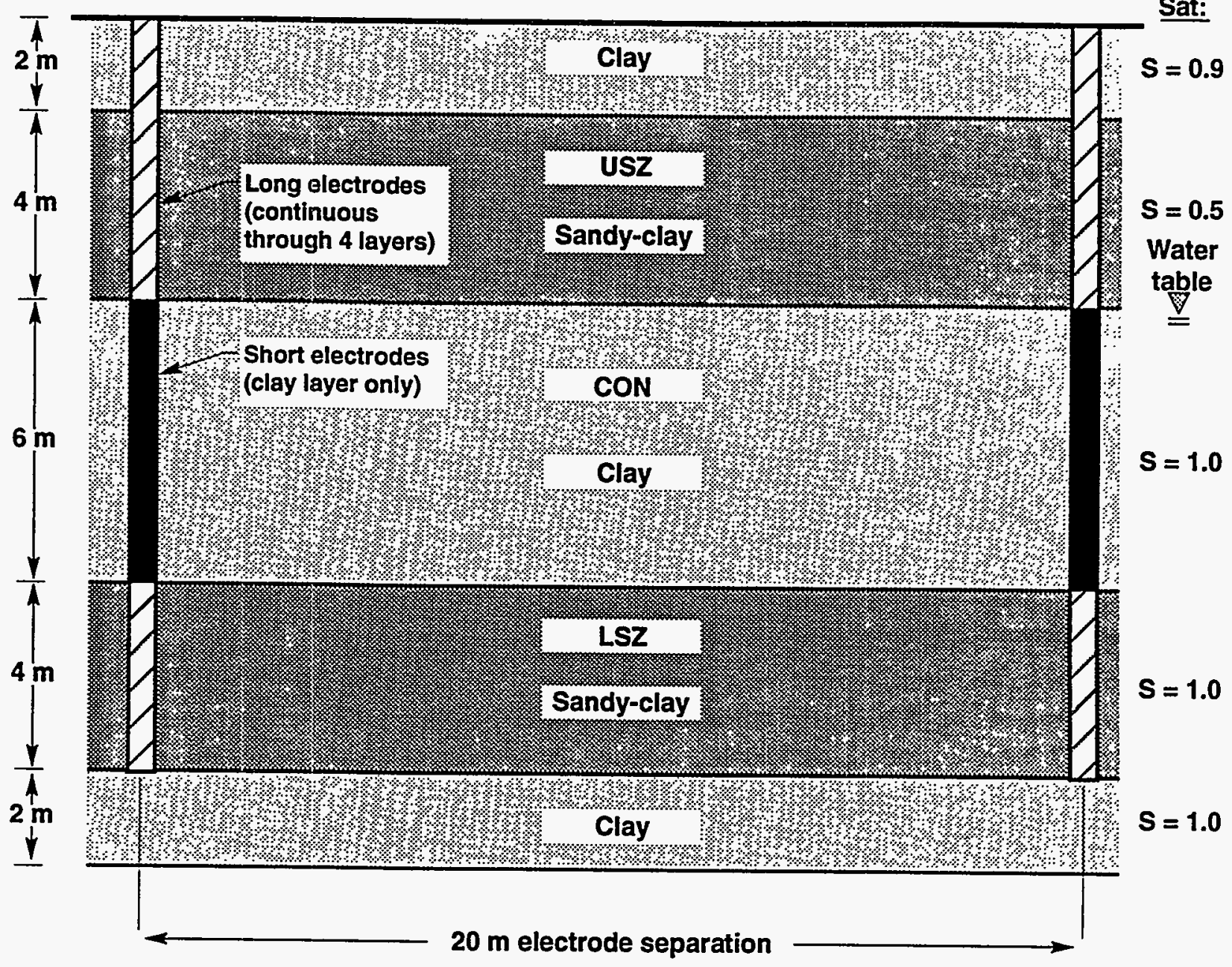

Figure A5-2. Simplified diagram of subsurface geology of heater wells at the TFF site which shows the size of the installed heater electrodes and their as-installed relation to the formation. The short electrodes (solid black) were installed and used during the DUSDP and the ARV phases of activities. The long electrodes (diagonal strips) were installed after the DUSDP in drill-back characterization wells and used exclusively during the ARV phase. 


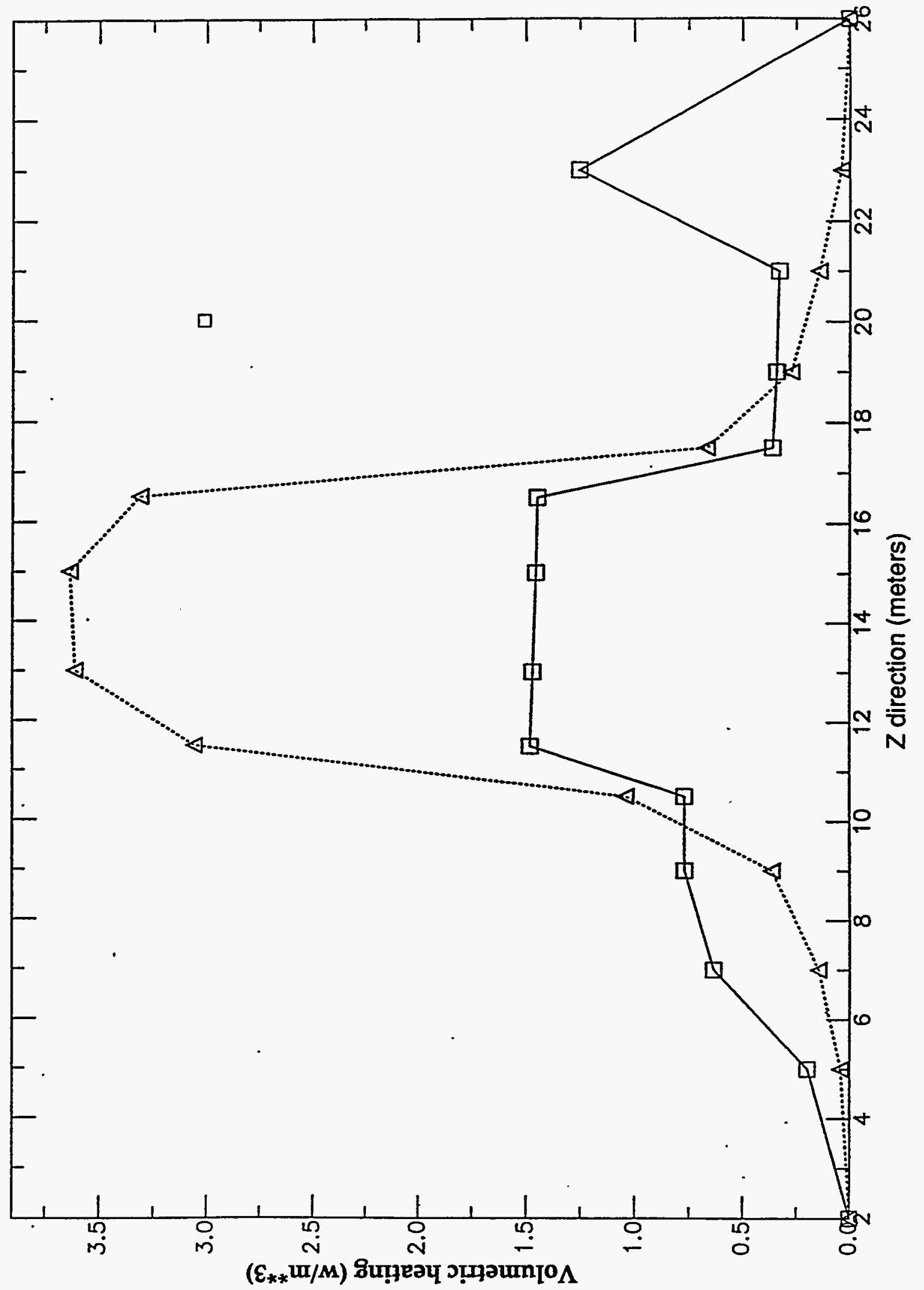


and, hence, the thermal energy is removed from the hydrologic system before it can influence the temperature of the central clay layer by conduction.

The five-layer model was used to examine the effect of steam production at the long electrodes. Unlike the short electrodes used in the clay layer, the long electrodes are porous along their length and readily permit the escape of steam that is produced in the electrode wells. To model the effect of steam production on ohmic heating, a small head of steam (approximately 3 psi) was maintained in the two electrode wells. The actual steam head is probably much higher, so that this model is conservative in terms of the effect of steam. Figure A5-4 shows the temperature and volumetric heating distribution in a vertical plane intersecting the two electrodes initially and at a time 5 days into a heating simulation. The temperature plots show a significant increase in the temperature of the partially saturated USZ as steam has gradually flowed into this zone (note the color change between the initial and final times in the upper part of the temperature distributions). The electrical conductivity has also been modified by the flow of steam into the USZ layer. This enhances the volumetric heating in that layer relative to the heating in the underlying clay layer, which is the target of the ohmic heating experiment. This can be seen by comparing the intensity of the color of the horizontal layer CON at the center of the plot to the intensity of the layer USZ immediately above the center. The darker layer at the earlier time becomes the brighter layer after 5 days of ohmic heating.

\section{Conclusion}

We have used numerical models to provide a diagnostic understanding of air sparging and the ohmic heating process. In the case of air sparging, we find that small inclinations of the injected layer tend to dominate the flow of the air plume. Even if large amounts of water are extracted, it is unlikely that the air plume can be made to flow downdip toward an extraction well. The modeling suggests that air sparging facilities must be carefully designed to take into account, through the placement of extraction and injection wells, the dominating effects of weak layer inclinations on determining the path of the injected air plume.

The various ohmic heating models that have been studied indicate that long, porous electrodes appear to produce effects that are counter to the goal of ohmic heating, which is to heat selected layers that are not amenable to infiltration by injected steam. For a given power input, long electrodes tend to divert current away from the target layers. When steam is produced at the electrodes, this diversion can be increased. For optimal heating of the target layer, it can be argued that any steam production should take place within the target layer and not at the electrodes themselves.

\section{References}

Waxman, M., and L. Smits, "Electrical Conductivities in Oil-Bearing Shaly Sands," Soc. Pet. Eng. J. (1968), 107-122, Transactions, AIME, 254. 

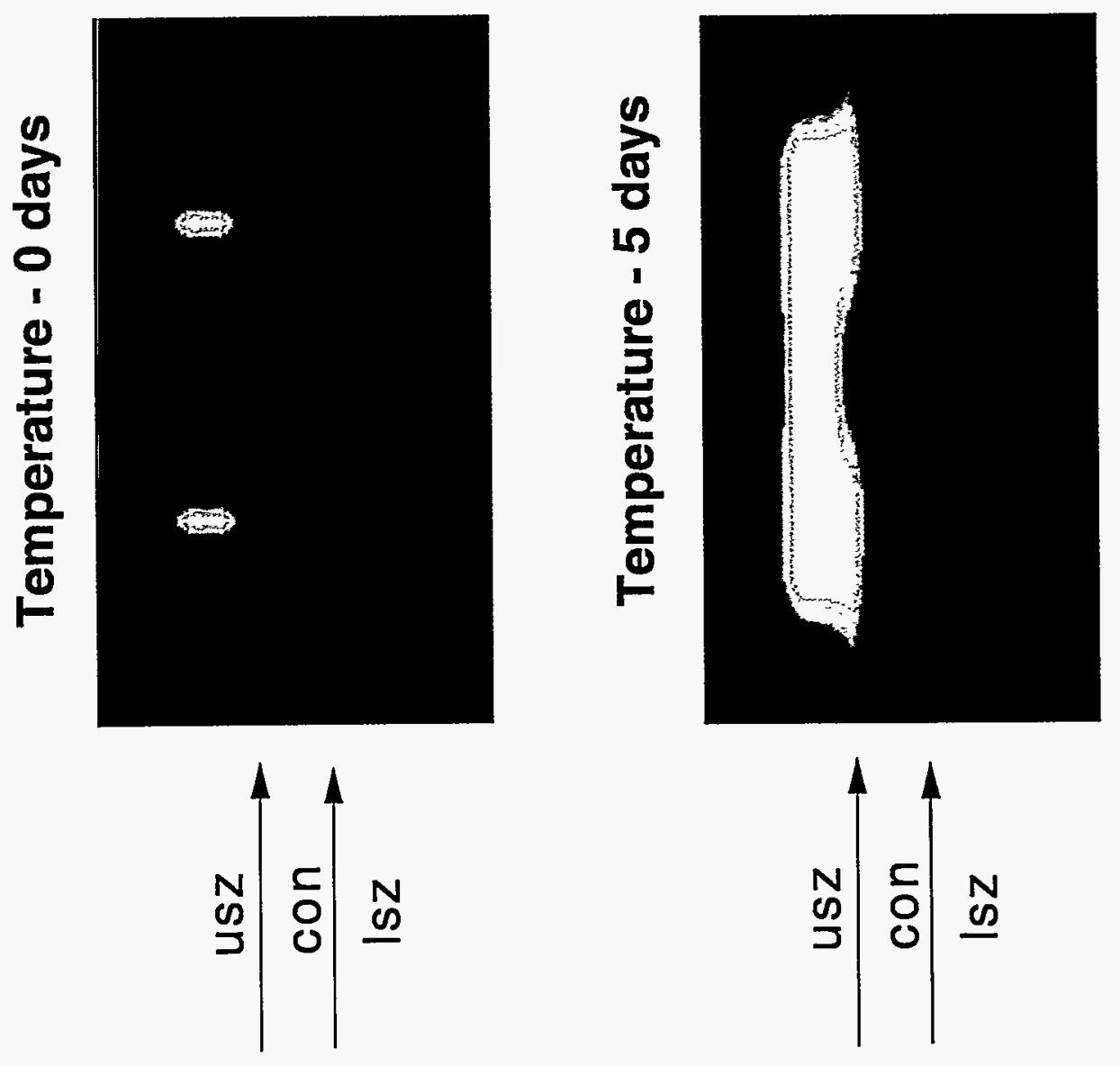

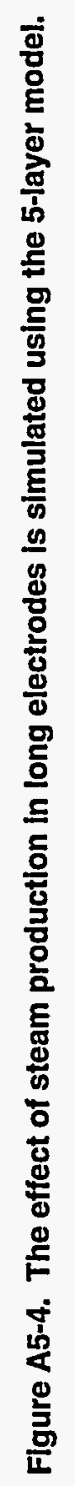
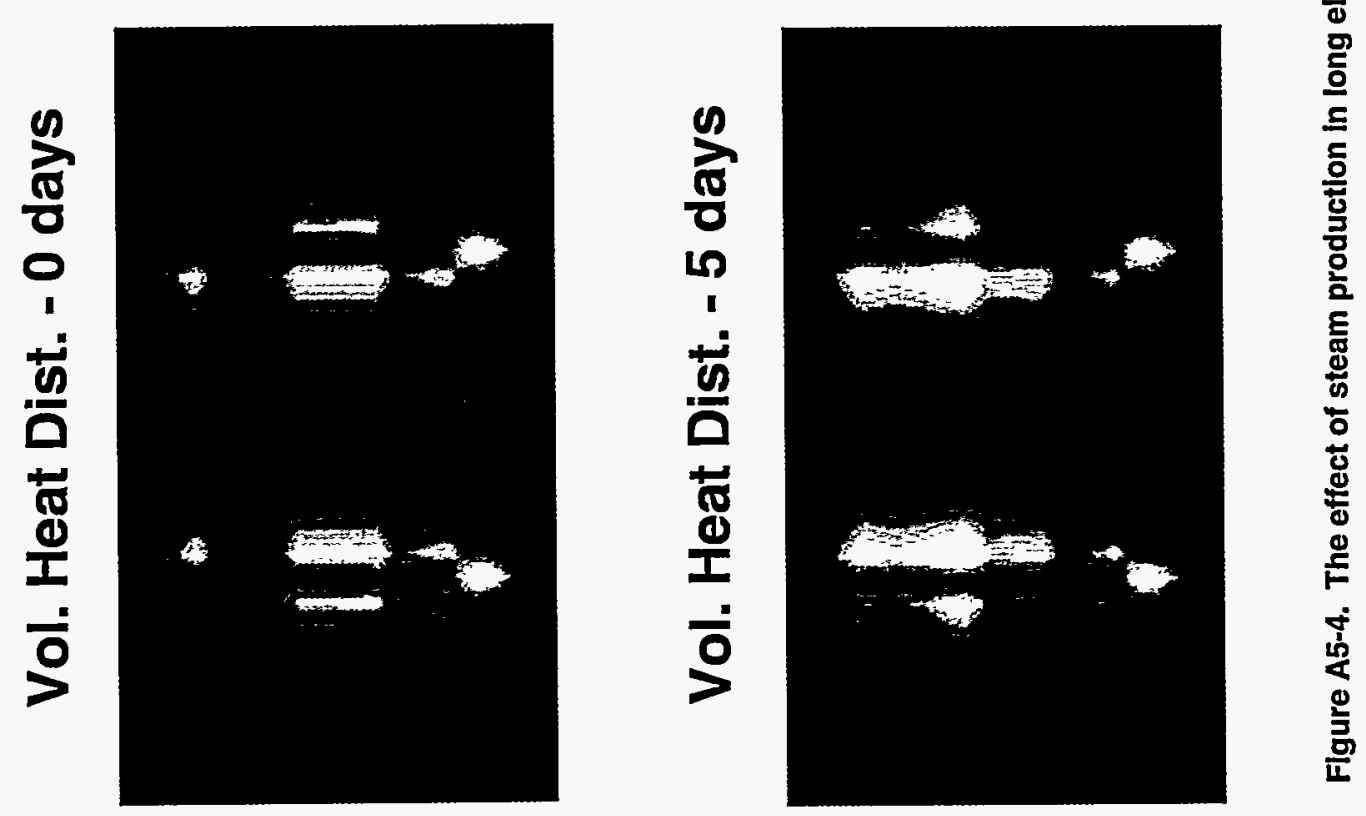


\section{Laboratory Determinations of Xylene Solubility as a Function of Temperature and Pressure}

\section{(Kevin Knauss, Earth Sciences Division, LINL)}

We made "reversed" (i.e., equilibrium approached from both super- and undersaturation) measurements of the liquid/liquid solubility of p-xylene in water as a function of temperature and pressure. Temperature was varied from $25^{\circ}$ to $150^{\circ} \mathrm{C}$ and pressure was varied from 15 to 7,500 psi. Experiments were run in high-pressure vessels modified to contain a flexible pure gold bag $\left(220 \mathrm{~cm}^{3}\right)$ fitted with a removable pure titanium closure. The high-pressure vessel was heated in a large tube furnace mounted on a rocking device, and was inverted to allow sampling of the aqueous phase. This device is ideally suited for mutual solubility measurements.

Sampling of the aqueous fluid was achieved by withdrawing through a titanium tube that exits through the head of the stainless steel high-pressure vessel and a special high-pressure valve (needle and valve block made of titanium) directly into a glass gas-tight syringe. The syringe is preloaded with methanol to maintain xylene in solution at room temperature and pressure. Pressure is maintained constant, even during sampling, by pumping water into the high-pressure vessel using a computer-driven precision HPLC pump, which collapses the gold bag.

The sample/methanol mixture was immediately ( $<2 \mathrm{~min}$ ) analyzed for xylene via GC using purge and trap extraction and detection via an FID (flame ionization). The GC analyses (including purge and trap processing) and data reduction are all accomplished using an HP chemstation. Calibration was via xylene standards prepared in methanol.

These data were used to calculate thermodynamic quantities pertinent to $\mathrm{p}$-xylene solution in water (Knauss and Copenhaver, 1994).

For this project, however, the desired quantities were the solubility of liquid xylene and the Henry's law coefficient $(\mathrm{H})$ as functions of temperature $(\mathrm{T})$.

The solubility of liquid xylene is determined directly by the measurements. These data are plotted in Figure A5-5. For the purposes of this project, the data are fit to a simple third order polynomial, and the regression results are displayed on the plot.

Knowing xylene solubility as a function of $T$, we can calculate $H$ via the expression:

$$
\mathrm{H}=\mathrm{pp} / \mathrm{x}
$$

where: $\mathrm{pp}=$ partial pressure of xylene at the temperature and pressure of interest

$$
\mathrm{x}=\text { solubility of xylene (as mol fraction) at the temperature and pressure of interest. }
$$

The pp of xylene at any temperature and pressure is derived from knowing the system total pressure and Pvp (the vapor pressure) of pure xylene. This is conveniently calculated using Antoine's equation:

$$
\ln \mathrm{Pvp}=\mathrm{a}-(\mathrm{b} / \mathrm{T}+\mathrm{c})
$$




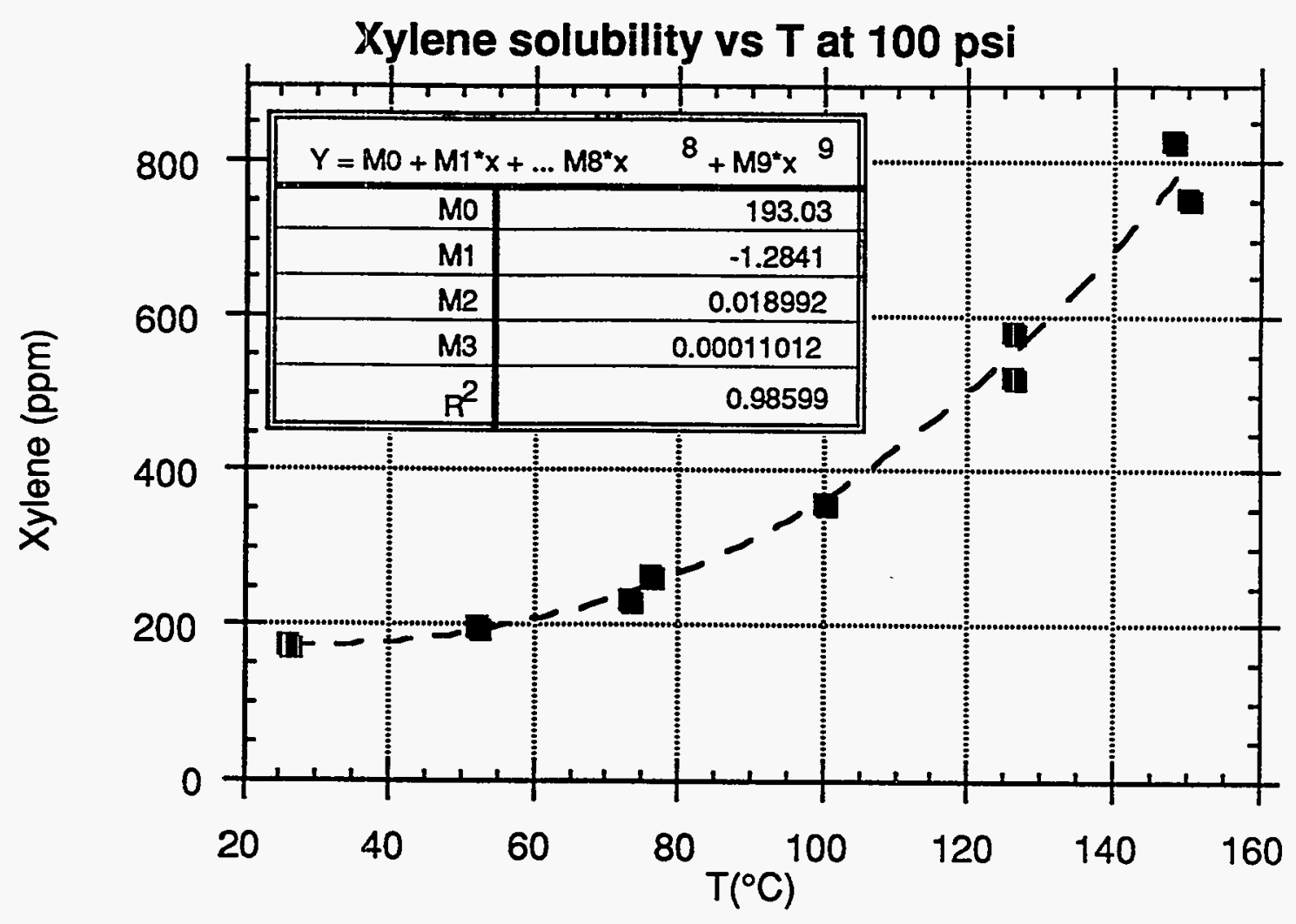

Figure A5-5. Experimentally determined (squares) xylene solubility as a function of temperature. 
and the appropriate constants $(\mathrm{a}, \mathrm{b}, \mathrm{c})$ extracted from any number of compilations (e.g. Reid et al., 1987).

Perhaps more directly useful than the Henry's law coefficient is the Dimensionless Distribution Coefficient (DDC). It can be shown (Knauss and Copenhaver, 1994) that with reasonable assumptions: $D D C=H / R T$, where $R=$ the gas constant and the other variables are previously defined. Figure A5-6 contains plotted values of $\log$ DDC vs T. The data are fit to a simple third order polynomial, and the regression results are displayed on the plot.

The reader wishing a more detailed accounting of the experimental procedures, the relationship of solubility to pressure, and the calculated thermodynamic quantities derived from the data is referred to Knauss and Copenhaver (1994).

\section{References}

Knauss, K. G., and S. A. Copenhaver (1994), "The Solubility of p-Xylene in Water as a Function of Temperature and Pressure and Calculated Thermodynamic Quantities," submitted to Geochim. Cosmochim. Acta. (also, Lawrence Livermore National Laboratory preprint UCRL-JC-117525).

Reid, R. C., J. M. Prausnitz, and B. E. Poling (1987), The Properties of Gases and Liquids, $4^{\text {th }}$ Edition, McGraw-Hill, Inc., N.Y., 741 pp. 


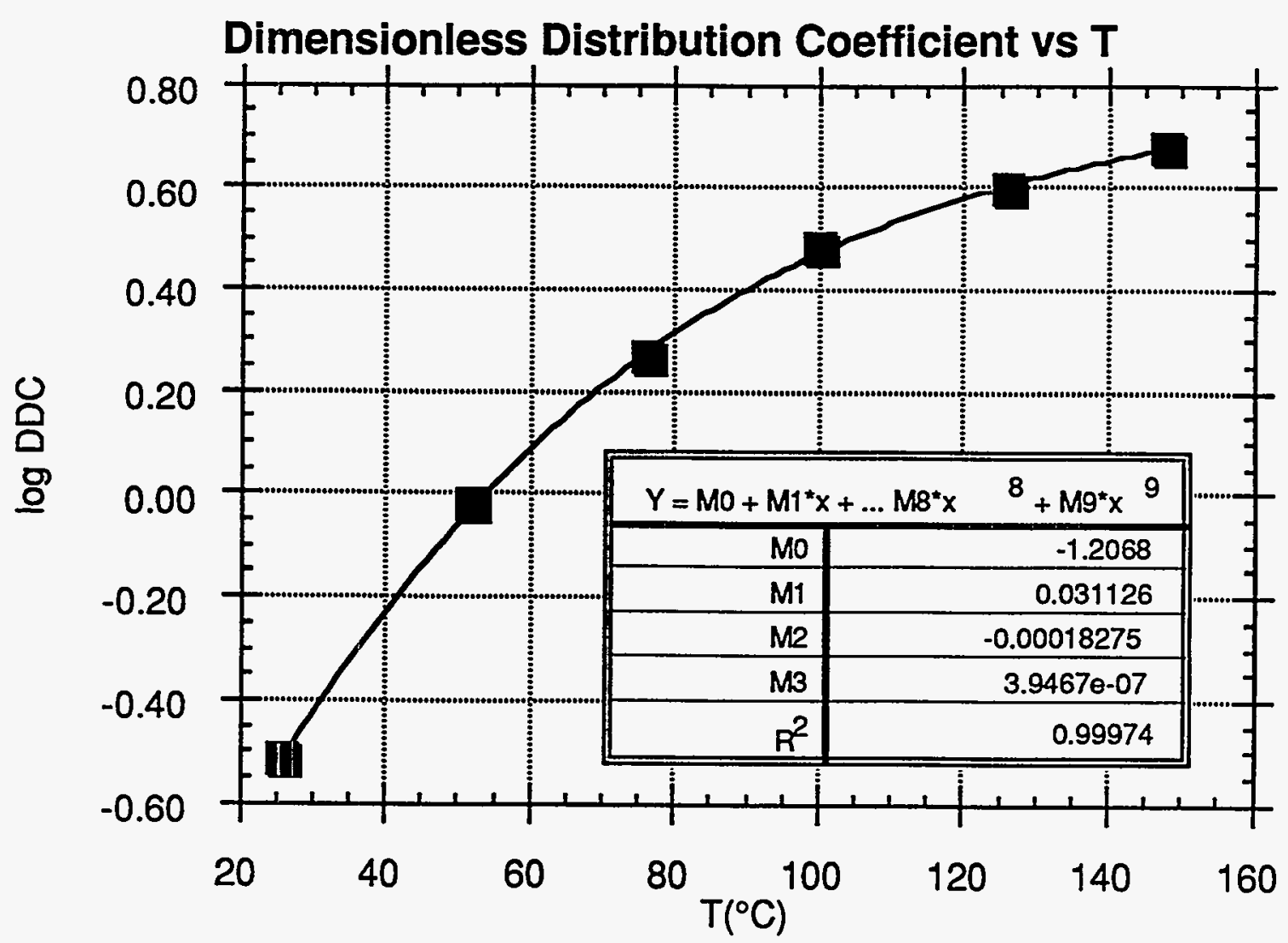

Figure A5-6. Dimensionless distribution coefficient as a function of temperature. 


\title{
Appendix 6
}

\section{Structure and Flow of Data through the LLNL ERD Environmental Data Management System}

\author{
(C. Suzanne Chamberlain and Patricia L. Ottesen, Environmental \\ Restoration Division, LLNL)
}

\section{Introduction}

This section describes the structure and flow of data in the data management system used by the Environmental Restoration Division (ERD) of the Environmental Protection Department (EPD) of Lawrence Livermore National Laboratory (LLNL) to store and archive data from the Accelerated Removal and Validation (ARV) Project.

The structure is based on two relational databases that are closely correlated. The first database handles sample tracking. The second database handles data such as sample location, media, analytical results, and some geological information (Fig. A6-1). These two databases are maintained on a VAX 6310 with VMS using INGRES relational database software. The flow of data, both hard copy and electronic, follows a model that tracks information from sampling plan through storage to archiving. The steps in the process include chain-of-custody $(\mathrm{CoC})$ tracking of the sample, analytical result receipt, the application of quality control procedures, and the electronic use of data in decision support tools, such as risk assessment and compliance monitoring.

\section{Structure and Flow}

A sample plan was developed to establish the frequency, method, and location of samples to be taken (Fig. A6-2). Field log books and CoC forms confirm the collection of samples according to the plan. A document control number is assigned to the samples based on the field log book used. A carefully controlled system of field log book labeling permits electronic tracking of an environmental sample from field collection through analytical result receipt as well as tracing back to the log book for any given analyte, should details of sampling conditions be needed. Samples are sent on to analytical laboratories where they are given unique log numbers.

The important fields in each SPACT record are document control number, analytical laboratory, analytical lab log number, sampling location identification, sampling date, and analysis requested. Dates tracked include those for receipt of the $\mathrm{CoC}$ form and analytical results, and date of entry. SPACT also includes invoice information. Receipt of official printed analytical results and invoices cause database records to be updated based on the document control number and location. A data record is marked complete only when all analytical results have been received. Thus, completion of a record confirms that all requested analyses have been performed and reported. 


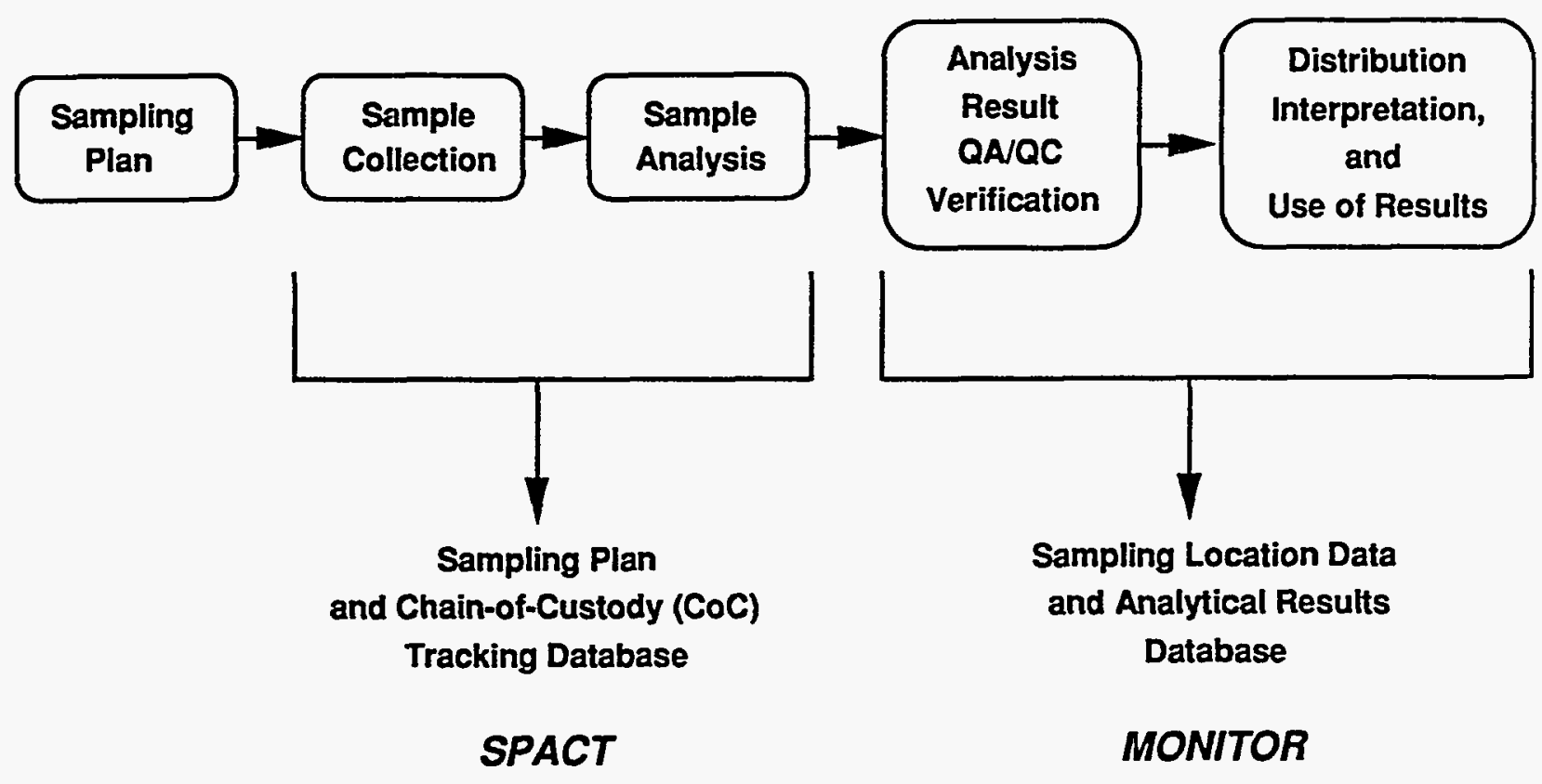

Figure A6-1. Environmental data flow. 


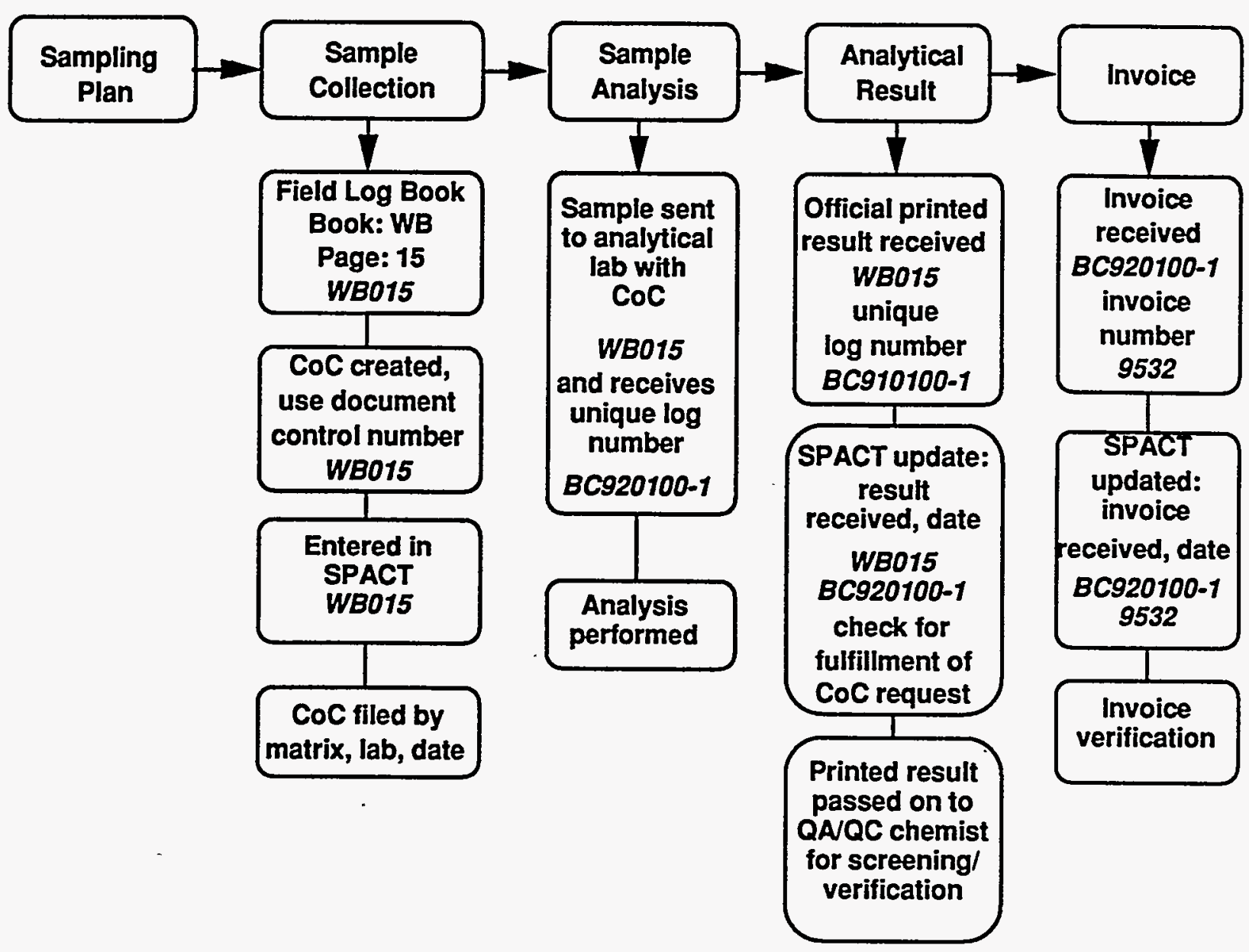

Figure A6-2. Environmental data flow - SPACT Database. 
Analytical results are stored in a separate, but correlated, relational database based on sampling location, log number, and date. This database is called MONITOR (Fig. A6-3) and is related to SPACT by identical fields: document control number, sampling location, sampling date, analytical laboratory, and requested analysis. Additional information collected for each sample and analyte includes requester, project, sample media, sample type, units, error, detection limit, dilution factor, and dates of extraction, analysis, and entry, together with comments and special notes. Sources of data in this database include geologic borehole logs, surveyor reports, field measurements, laboratory measurements, calculated or reduced data, and test conclusions. Types of data to be stored have included descriptive sample location information, such as coordinates, elevations, lithology, and screened intervals of monitoring installations, as well as measurements and analytical information, including physical and chemical parameters, media identification, and ground water elevation measurements.

Data verification and validation are achieved through a combination of methods. Handentered data are run through a series of computerized verifications that check for duplication, empty fields, and reported results not consistent with reported detection limits. Data are also thoroughly checked by a second person before being formally added to the database. Electronically delivered laboratory data are groomed to fill in empty fields and insure consistency in fields such as sample location, project, media, and type. Computerized verifications are also run on electronic data, and a second person checks sample descriptor fields before formal addition to the database. Random audits are done to verify electronically delivered results against official printed results. Analytical results added to the database are validated through review by qualified chemists. Final verification is done by project scientists who request and use the data. They report outliers, anomalies, and any other inconsistencies indicated by comparison with similar data and historical trends to both the data management team and the quality control chemists who review and qualify or correct the data as appropriate.

The MONITOR database also contains fields dedicated to quality control. Such fields include flags, indicating analytical result qualification and data quality level. The result qualifier flags are absent from a routine report, but may be included to show nondetection, dilution greater than one, rejection, or any of several other types. Data quality levels can range from EPAapproved methods performed by a certified laboratory to quick, approximate field analyses.

Printed copies of the data received are filed by location and chronological order in a data reference library. The originals are retained for data submittal to regulators when required.

The operations data are stored in spreadsheets on Macintosh computers at the Gas Pad. Complete backups on this data occur on a weekly basis with incremental backups occurring daily. These weekly backups are currently stored in T4383. Plans are being developed for fireresistant storage. To allow for long-term access to the backed-up data, each month a tape is marked to save until data generation ceases. At the time data generation ceases, three complete archival backups will be performed and stored in separate locations. This procedure will provide the highest level of availability of the data in the future. 


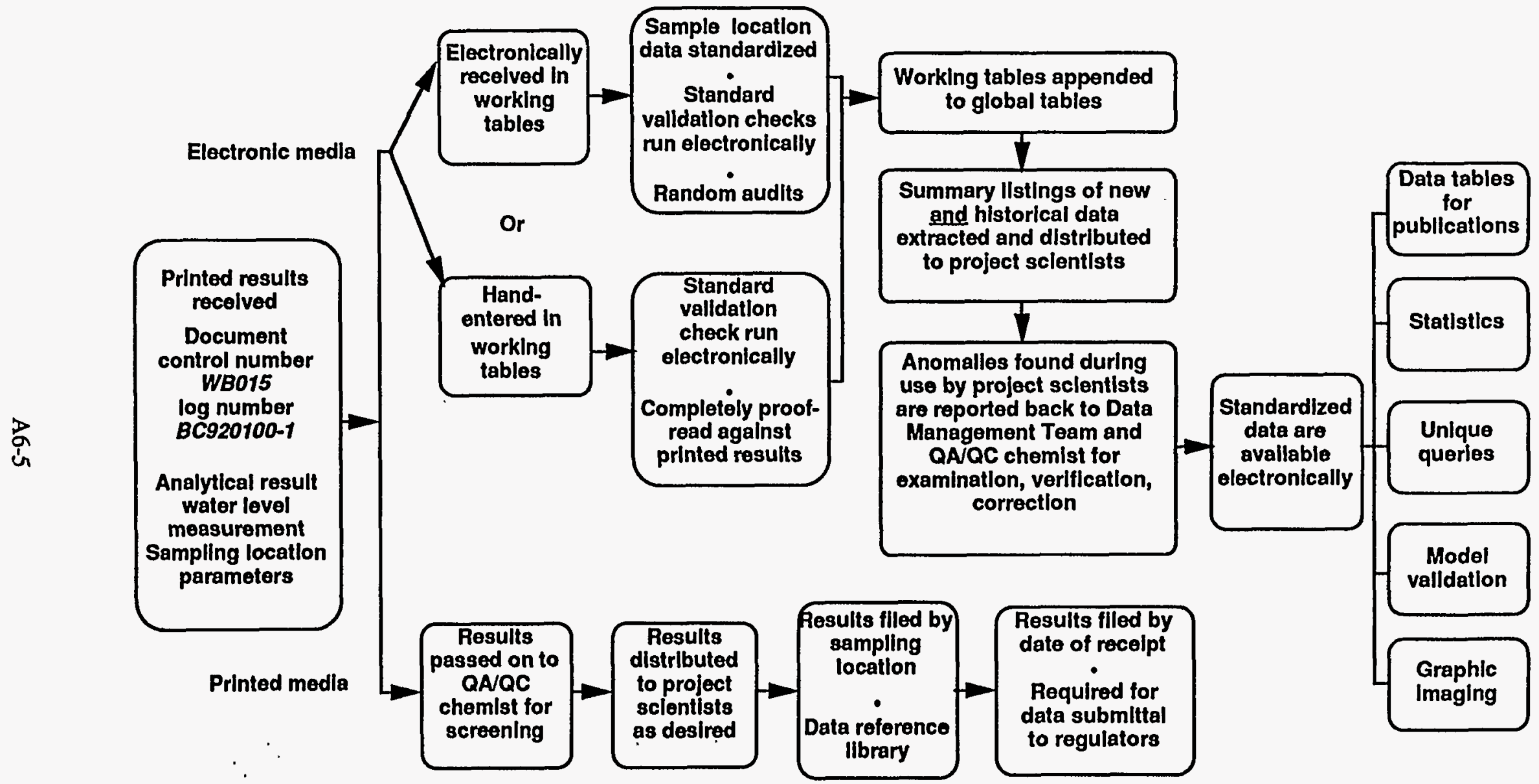

Flgure A6-3. Environmental data flow - MONITOR database. 


\section{Conclusions}

There are many advantages of this integrated centralized data management system. The use of such a system promotes and provides a consistent data set of known quality, which is available to all. Single entry for multiple use allows quality assurance and quality control to be performed equally for all data. Once an error is discovered and corrected, the data will be correct for all future uses. Interesting correlations and trends in the data may become obvious from linked extractions in the future. 